\title{
Dynamic Tethering for Enhanced Remote Control and Navigation
}

\author{
Herman W. Colquhoun Jr.
}

\begin{abstract}
A thesis submitted in conformity with the requirements for the degree of Master of Applied Science
\end{abstract}

Graduate Department of Mechanical and Industrial Engineering

University of Toronto

January

2000 
National Library

of Canada

Acquisitions and Bibliographic Services

305 Wollingtion Street Ouman ON K1A ONA Canede
Bibliothèque nationale du Canada

Acquisitions of services bibliographiques

305, no Wollington

Otinwa ON K1A ONA

Cenada

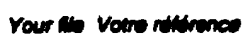

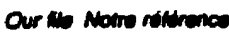

The author has granted a nonexclusive licence allowing the National Library of Canada to reproduce, loan, distribute or sell copies of this thesis in microform, paper or electronic formats.

The author retains ownership of the copyright in this thesis. Neither the thesis nor substantial extracts from it may be printed or otherwise reproduced without the author's permission.
L'auteur a accordé une licence non exclusive permettant à la Bibliothèque nationale du Canada de reproduire, prêter, distribuer ou vendre des copies de cette thèse sous la forme de microfiche/film, de reproduction sur papier ou sur format électronique.

L'auteur conserve la propriété du droit d'auteur qui protège cette thèse. $\mathrm{Ni}$ la thèse ni des extraits substantiels de celle-ci ne doivent être imprimés ou autrement reproduits sans son autorisation. 


\title{
Dynamic Tethering for Enhanced Remote Control and Navigation Herman W. Colquhoun Jr. \\ Ergonomics in Teleoperation and Control Laboratory \\ Department of Mechanical and Industrial Engineering \\ University of Toronto, Canada
}

\begin{abstract}
Misalignments between display and control reference frames complicate task execution by loading the operator's attentional resources with mental transformations. It is thus important to maintain alignment between an operator's controls and her view of the controlled object or task space. Maximising the operator's situational awareness within this task space by providing an optimal frame of reference also simplifies task execution. Traditional rigid tethering integrates the desirable egocentric and exocentric aspects of a display by connecting the exocentric view of the task space to the telemanipulator. Dynamic tethering (a superset of rigid tethering) preserves the principle of the moving part that is violated when the rigid tether is implemented, while maintaining controldisplay motion compatibility.
\end{abstract}

The human operator information-processing model may be split into three stages: perception, decision-making, and response control. If additional transformations must be performed to produce an appropriate response then the rate at which the information is handled within a particular stage will be below maximum. The need to perform complex data transformations imposes a particular demand upon the decision-making stage. In general, reducing the degree of processing required to map input information into a desired response improves information transmission rate and thus, improves control performance. The concept of tethering attempts to minimise any additional processing 
related to mental rotation (as encountered in a non-tethered display) and scene integration (as required by an egocentric display). Dynamic tethering maintains the advantages of traditional tethering while minimising the need to compute the derivatives of displayed information to determine the effects of previous responses. The results of this study reveal that dynamic tethering reduces the frequency of control reversals and the reaction time associated with docking task execution. At near optimal tether natural frequencies sine-wave tracking performance with a tethered interface exceeds that obtained with either traditional non-tethered or rigidly tethered displays. 


\section{Dedication}

This thesis is dedicated to my newborn son

Caynen Warren Colquhoun

Born: November 2, 1999 


\section{Acknowledgements}

I would like to acknowledge the PRECARN Associates and MacDonald Dettwiler Space and Advance Robotics Ltd. (formerly Spar Aerospace Limited) for their support of this research. In particular, I would like to thank Eldon Wong and Ross Gillett of MacDonald Dettwiler Space and Advance Robotics Ltd. for their assistance in setting up these experiments and providing the facilities and equipment required to perform them. 


\section{Table of Contents}

ABSTRACT I

1. SUMMARY 1

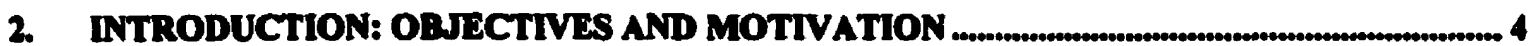

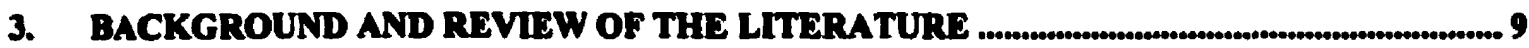

3.1 LINKING REMOTE EXTERNAL SPACE TO INTERNAL SPACE ....................................................... 10

3.1.I Mapping the Remote Hand to the Mind ...................................................................................... 10

3.1.2 The Role of Teleproprioception and Telekinesis in Remote Control .................................... 13

3.I.3 Proprioception and Sensori- Motor Control ........................................................................ 17

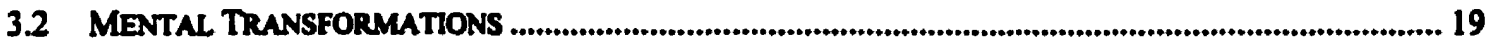

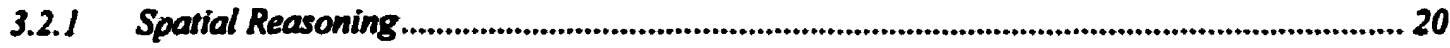

3.2.2 Mental Rotation and the Forward Direction ........................................................................ 23

3.2.3 Mental Rotation in 3 Dimensions .......................................................................................... 29

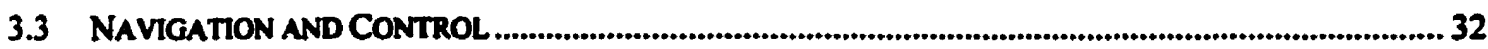

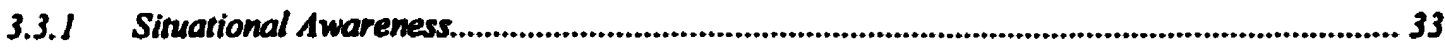

3.3.2 Spatial Compatibility .............................................................................................................. 34

3.3.3 Spatial Awareness, Navigation, and Control.................................................................. 39

3.3.4 The Effect of Incongruency on Navigation ........................................................................... 45

3.3.5 The Effect of Incongruency on Control ............................................................................... SI

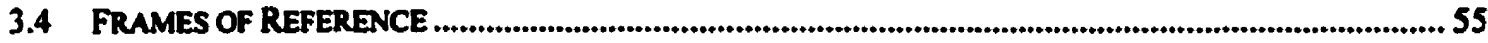

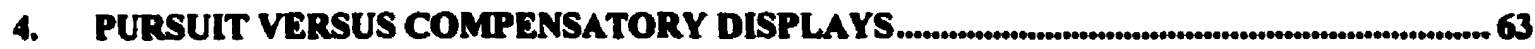

4.1 DISTINCTIONS BETWEEN COMPENSATORY AND PURSUIT DISPLAYS..............................................63

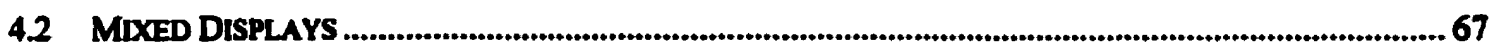

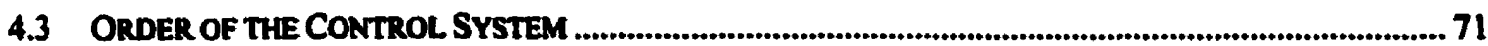

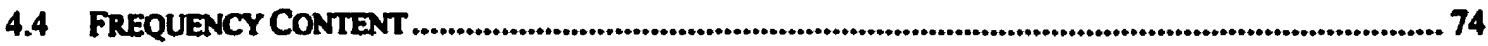

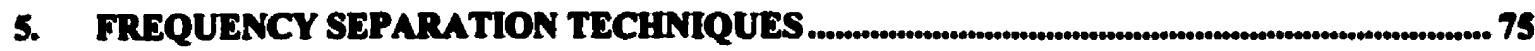

5.1 THE PRINCIPLE OF FREQUENCY SEPARATION..................................................................................

5.1.I Aviation Research involving Pursuit, Compensatory, and Frequency Separated Displays . 76

5.1 .2 Outside the Cockpit............................................................................................................................... 81

5.1.3 Information-Processing Models and Control Theories ........................................................... 83

5.1.4 Motion perception and Apphying the Frequency Separation Technique............................... 89 


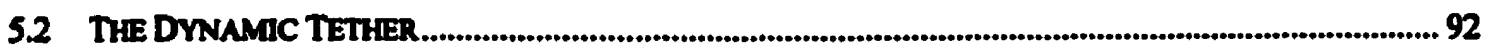

S.2.I Modelling the Dynamic Tether ................................................................................................. 92

S.2.2 Dymamic Tethering and the Proximity Compatibility Principle .......................................... 103

5.2.3 Motion Detection ................................................................................................................. 104

5.2.4 Some Examples of Dynamic Tethering ................................................................................ 107

5.2.5 Design Trade-offs: Changing the Tether's Dymamic Response Characteristics.................. 107

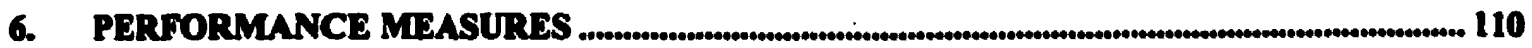

6.1 TRACKNG A QUASt-RANDOM SANE WAVE TRACK................................................................ 110

6.2 MEASURES OF PERFORMANCE FOR SINGLE STEP TRACKING...................................................... 113

6.3 MEASURES OF PERFORMANCE FOR DYNAMIC TRACKING.......................................................... 115

6.3.I Measures of Performance for Simusoidal Tracking ................................................................. I16

6.3.2 Averaged Mearures of Error ...................................................................................................... II7

7. JUSTIFICATION AND HYPOTHESES FOR A NOVEL STUDY ............................................. 119

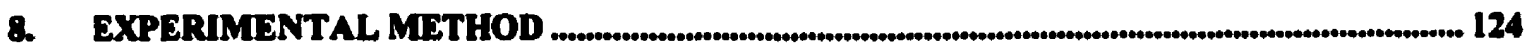

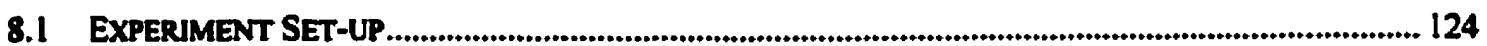

8.1.I Experimental Platform and Apparatus ................................................................................ 124

8.1.2 Experimental Displays and Tasks...................................................................................... 126

8.1.3 Optimisation of Control Gains ............................................................................................. / /33

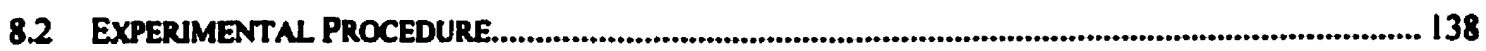

8.2.1 Experimental Design...................................................................................................... 139

8.2.2 Participants.................................................................................................................. $/ 42$

8.2.3 Procedure ................................................................................................................... $/ 43$

9. EXPERIMLNTAL RESULTS AND DISCUSSION ..................................................................................... 14S

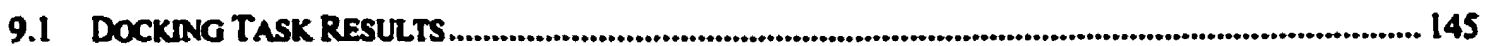

9.1.I Task Completion Time ...................................................................................................

9.1 .2 Percent Overshoot ................................................................................................................. 151

9.1 .3 Reaction Time.............................................................................................................. 156

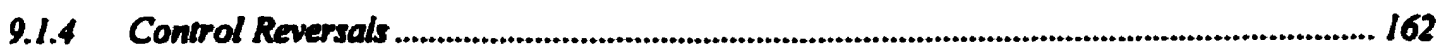

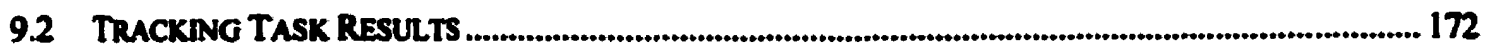

9.2.1 Tracking with the Plan Viewpoint................................................................................ 172

9.2.2 Tracking with the Perspective Viewpoint................................................................................ 177

9.2.3 Spectral Analysis of Tethering Effects on Performance ........................................................ 179

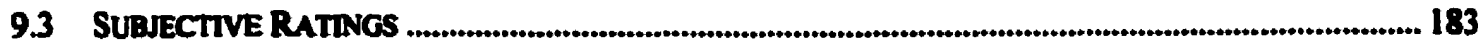




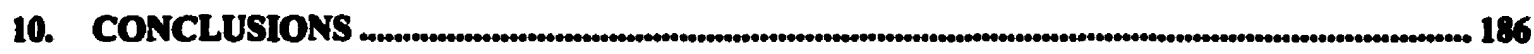

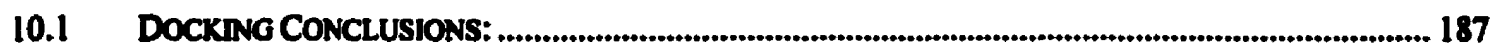

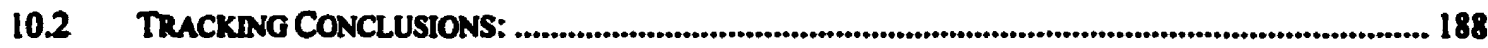

10.3 CONCLUSIONS BASED ON SUBJECTIVE EVALUATIONS:........................................................... 190

11. RECOMMISNDATIONS ....

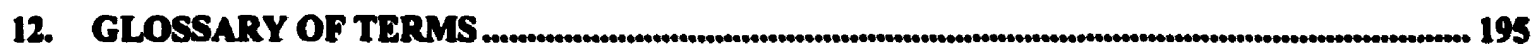

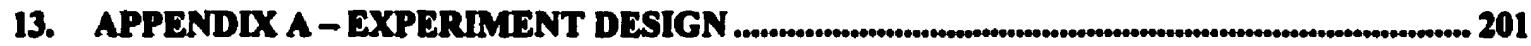

14. APPENDIX B - RESULTS OF ANOVA AND MULTI-FACTOR CONTRAST TESTS ....... 202

15. APPENDIX C - SUBJECT INFORMATION PACKAGE $\ldots$

16. APPENDIX D - SPREADSHEET: CALCULATION OF FORCING FUNCTION

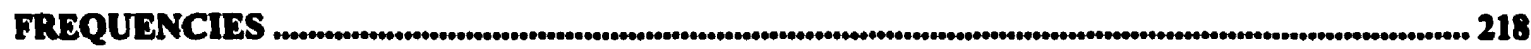

17. APPENDIX E - NUMERICAL METHODS USED IN MODELLING THE DYNAMIC TETHER

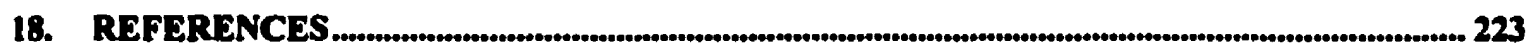




\section{Summary}

Navigation and control of remote devices, be they endoscopic-surgical tools or those that enable a driver to keep a remote excavator upright, are critical to successful task completion and can weigh heavily on the attentional demands of the operator. It is therefore important to design displays that provide appropriate feedback about these devices and their environment and support these tasks without adding too much complexity. It is also important to maintain adequate alignment between an operator's controls and her view of the controlled object, because misalignments between display and control reference frames complicate task execution by loading the operator's attentional resources with mental transformations. Another objective of display design is to maximise the operator's global situational awareness within the task space. This may be achieved by providing an optimal frame of reference for a given task. In general, this is best resolved with an exocentric view. It is widely recognised that global awareness increases with exocentricity while the awareness needed for local guidance decreases accordingly.

Tethering integrates the desirable aspects of egocentric and exocentric displays by linking the exocentric view of the task space to the telemanipulator. Wickens (1994) described this link as a "3D tether". This virtual "camera-manipulator" connection allows both global awareness and local guidance information to be processed simultaneously. Egomotion within the resulting display ensures that control-display congruence is maintained. Also, because the attached viewpoint is exocentric, global situational awareness is promoted. On the other hand, local navigational awareness is also preserved 
since the viewpoint is only slightly displaced from its "nominal" position while the egomotion (reflecting changes in ego-position of ownship) maintains this relationship. However, the rigid tether violates the principle of motion compatibility, as the operator will observe motion which is opposite to the direction of her input. In addition, because the output of such a display closely resembles that of a compensatory tracking system, certain well-known disadvantages associated with cue depletion may result.

It is proposed that relaxing the constraints associated with the "rigid" tether may help to alleviate some of these problems. Modelling the tether as a spring-damper system allows its rigid character to be modified. This dynamic tether creates a display that combines elements of both compensatory and pursuit tracking systems. Early work by Senders and Cruzen (1952, as cited by Roscoe et al., 1981), indicated that "a good deal might be gained by using a display that has the target ... move slightly yet keeps the scaling advantages of compensatory presentations". Fogel introduced the "frequency-separation" concept in his Kinalog display in 1959, and Roscoe conducted further research in the 1970's. In essence, the dynamic tether is an example of frequency separation.

In the present study a tether is modelled in a virtual environment and connects the viewpoint to the controlled object. The model for the tether is based on the dynamic equations for a mass spring-damper system. Five tether models were used to represent the varying degrees of a continuum of tether characteristics from one extreme - very loose - to the other -- very rigid. Subjects were required to perform both docking and tracking tasks using the various tethers. Various objective and subjective measures were 
collected as measures of performance including percent overshoot, reaction time, frequency of control reversal, completion time, RMS error, and subjective measures of understanding and usability.

Dynamic tethering maintains the advantages of traditional tethering while reducing the need for the computation of derivatives of presented information. The results of this study reveal that dynamic tethering minimises reaction time and the frequency of control reversals. Also, at near optimal tether natural frequencies sine-wave tracking performance with a tethered interface surpasses that obtained with the traditional nontethered (pursuit-like) or rigidly tethered (compensatory-like) displays. 


\section{Introduction: Objectives and Motivation}

Good old ideas for pictorial flight displays that were once impractical warrant reconsideration in light of current microcomputing and display technology. Among the ideas are the contact analog, highway in the sky, and flight path predictor concepts. Basic pictorial display principles established in the 1950s and 1960s have been supported by additional experimental findings in the 1970s. These include pictorial realism, magnification; integration, compatible motion, frequency separation, pursuit presentation, quickening, and predicting.

- Roscoe, Corl, and Jensen, 1981.

Roscoe et al. (1981) refer to a large body of research that has been conducted in the 1960's and 1970's which support the use of various 'ideal' tools to improve aeroplane cockpit instrument and display design. Some of these ideas do not apply solely to cockpit design but may be extended to many other paradigms, from micro-surgery to space robotics. But it has been a while since the time of Roscoe's article and we can now also include the ideas generated and the research conducted in the 1980s and the 1990s to this list. Much of the research performed with respect to frequency separated displays (FSDs) in the past has been related solely to avionics (Fogel, 1959; Roscoe, 1973; Roscoe, Corl, and Jensen, 1981; Beringer, Williges, and Roscoe, 1975; Bauershmidt and Roscoe, 1960).

The principle of frequency separation refers to an algorithm that permits rapid changing display properties to be driven by an outside-in principle while relatively low frequency changes are driven according to inside-out properties. These concepts are illustrated in the context of the aircraft attitude-direction indicator in Figure 1. The outside-in display is illustrated as a moving aeroplane indicator while the inside-out display corresponds to the traditional moving horizon indicator. The concept of frequency separation is 
illustrated as the transition between the two displays (the indicator in the middle of Figure 1).

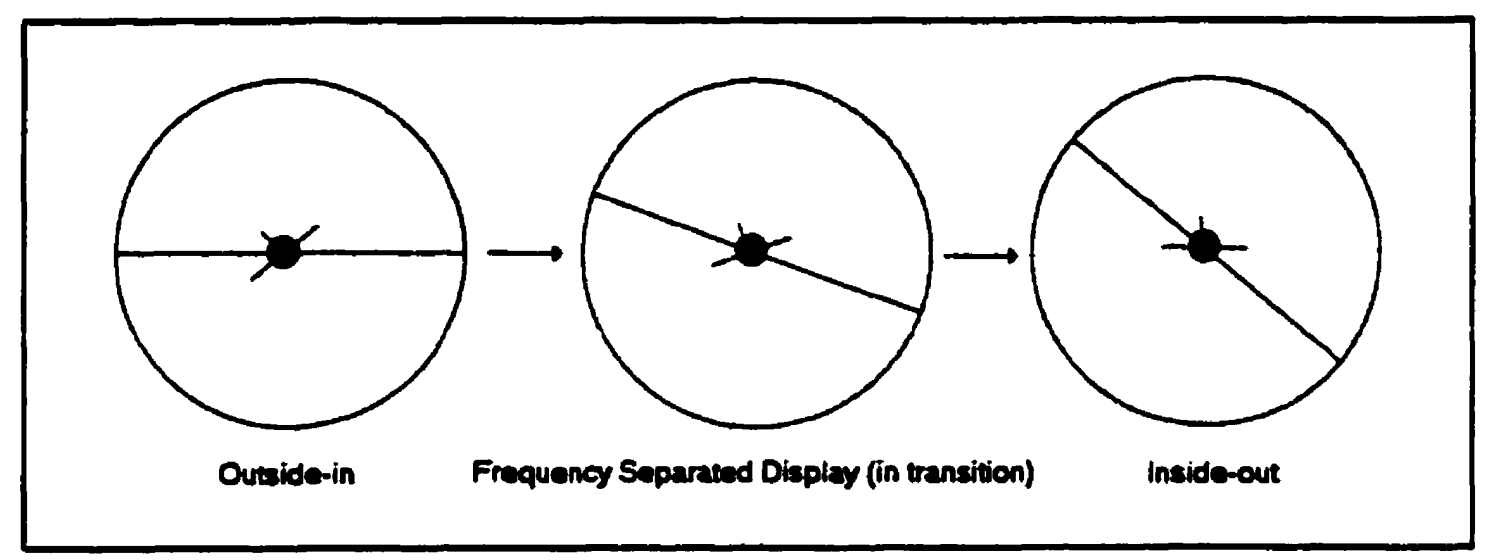

Figure 1 lllustration of the outside-in, frequency separated and inside-out concepts in the context of an attitude-direction indicator.

One of the goals of this research is to illustrate the potential for frequency separated display (FSD) technology to be extended to other paradigms. In particular, many teleoperation tasks may benefit from the implementation of FSD technology. Virtual reality and mixed reality (Milgram \& Kishino, 1994) have made the integration of such advanced display tools into cockpits, control stations and other video terminals a reality. For example, teleoperation is frequently performed while tolerating misalignments between the operator or camera viewing orientation, and the controller orientation. It is important to design displays that provide appropriate feedback about the controlled devices and their environment, and to support these tasks without adding too much complexity. Some examples of teleoperation scenarios that may benefit from the use of such advanced display tools are endoscopic surgery, space robotic operations, and toxic waste handling robotic operations. It is important to maintain adequate alignment between an operator's controls and her view of the controlled object, since misalignments 
between display and control reference frames complicate task execution by loading the operator's attentional resources with mental transformations.

In the aforementioned examples operator attention is a valued resource and anything that takes away from his ability to devote his full attention to the task can have costly repercussions. Thus, an effort should be made in display design to maximise the operator's global situational awareness within the task space by providing as close as possible to an optimal frame of reference for a given task. In general, this is best achieved with an exocentric ${ }^{l}$ view. It is widely recognised that, although global awareness increases with exocentricity, the awareness needed for local guidance decreases accordingly. A more detailed discussion of these concepts and the history that precedes them are presented in the literature review in the next section.

The objectives of this study are twofold:

(1) To evaluate human manual docking and tracking performance while using a dynamic tether to couple the virtual scene with the camera viewpoint, a novel FSD related technique of mixing compensatory and pursuit display characteristics as a function of the user's input control frequency; and

(2) To detect any quantifiable differences in performance as a function of the natural frequency of the dynamic tether (i.e., the location of the nominal breakpoint used when mixing compensatory-pursuit information in the frequency domain). 
The results of this study may be used to guide display design in various teleoperation environments comprising either virtual or mixed reality content. The implementation of such findings should reduce the frequency of control errors and navigational mistakes, while increasing the accuracy and mental retention of task execution and task details, respectively.

Using the current findings, the principle of frequency-separation may be used to produce solutions to both display motion relationship problems, as well as control-display congruency problems. The mode, function, and format of any display all mediate its efficacy for presentation and assimilation of system information. The human perceptual system has evolved to deal with objects and events available to direct observation in everyday life (Gibson, 1979 as cited by Stokes et al., 1990). Displays, however, filter, aggregate, delay (or predict), and otherwise transform system information in ways that may not always be compatible with the perceptual system that evolved in human beings. Furthermore, displays may provide far more information than would otherwise be available through direct observation and can thus potentially overload the human operator with more system parameters than can be efficiently processed and integrated within the available time. In such cases, performance may then become "resource limited", that is, the limits of an individual's cognitive resources constrain the level of performance that can be achieved. Inefficient data representations may not always be due solely to the form of the display, however, but in fact also to the natural form of the information. Displays should thus be designed to augment display interaction, improve the form of the 
data representation, and reduce the mental workload involved in performing a task. The dynamic tether may be one such vehicle that could be used to achieve these goals.

'The term centricity refers to the extent to which a human observer's viewpoint is removed from the "ownship", that is, from the nominal viewpoint with respect to the viewer's own avatar, or own vehicle, or own manipulator within the task space. 


\section{Background and Review of the Literature}

The current study investigates display frame of reference issues associated with user navigation and control of teleoperators ${ }^{2}$. The chosen frame of reference for a telemanipulation task is a very important influence on human performance in many applications. Relevant applications include space operations (NASA Ames, 1998), aviation (Johnson and Roscoe, 1972), remote vehicle control (McGovern, 1991; Kappé, 1997; Craig, Hartzell, and Dunbar; Milgram and Colquhoun 1999a), scientific visualisation (McCormick et al., 1998), endoscopic surgery (Holden, Flach, and Donchin, 1999, Milgram and Colquhoun, 1999b), land navigation (Glumm et al., 1998; Kitamura et al., 1998), and telerobotics (Sheridan, 1994). In the particular domain of aviation, the frequency separation principle (FSP) has been proposed as a potential means of alleviating some kinds of frame of reference problems. In this chapter a review is given of some of findings of past FSP and related studies in aviation, with an eye to how the principle may be applied to the domain of teleoperated ground vehicle control and navigation in virtual environments. The following sections describe the main concepts that provide evidence in support of the need for tools such as frequency separation algorithms in ground-based teleoperation and navigation within virtual environments. The impact of the operator's ability to map the remote task space to his mental representation of that space, his ability to perform mental spatial manipulations and the importance of the frame of reference with respect to effective task execution are discussed. 


\subsection{Linking Remote External Space to Internal Space}

The concept of congruency refers to one-to-one correspondence between the display and the controls. When congruency is attained control inputs result in a corresponding change in the display in the direction expected with respect to the other control and display variables. The following sections describe the relationships between the mental / spatial representation of the remote environment and display properties and the importance of utilising the body's natural senses to improve task performance in teleoperated environments. Mental mapping of the remote manipulator refers to the ways in which relationships between controls and displays are expected and/or learned, and how these relationships can be violated. Teleproprioception, telekinesis, and proprioception in motor control are important in terms of monitoring control output and building or correcting mental and physiological relationships between the local body's actions and its effects on the remote environment. Together the literature on these topics provides guidelines and support for the design of more efficient displays for teleoperation based on the preservation of control-display congruency.

\subsubsection{Mapping the Remote Hand to the Mind}

The arrangement of what the operator sees, what she does with her hands, and how this corresponds with what she thinks is critical, and is the basis of the human factors notions of "display-control compatibility", "user expectation stereotypes", "naturalness", and "transparency". Sheridan (1994) comments on the difficulty in designing humancomputer interfaces with respect to these concepts: "Miscorrespondence ([the] inability or difficulty in mapping from eye or other sensor to mind, and then to hand) is likely to 
cause delays and errors. [A] computer's internal models will run at their own speed and in terms of whatever the programmer decided, but these must bear correspondence to the mental and display-control models. All, of course, must correspond to the external reality. Designing in this correspondence is not a trivial matter".

In a highly relevant study, Holden, Flach, and Donchin (1999) examined the perceptualmotor co-ordination with an apparatus that simulated a surgical endoscope. The participants were trained with one arrangement of the apparatus, then tested with another configuration, in which the positions of the camera, the surgeon, or the objects in the surgical field of view were altered relative to each other. The results of this study showed that changing only either the camera or the surgeon's position disrupted performance; but, when the two were moved together in a synchronous fashion, skilled performance was preserved. This suggests that skill is highly dependent on a consistent mapping between the (virtual) hand and eyes, but not necessarily on the particular visual or motor orientations.

When designing dynamic displays or interfaces in general, compatibility is always a concern, i.e., compatibility between the operator's mental model of the displayed information and the actual displayed information itself. There are three types of compatibility for dynamic analogue displays (Liu, Chapter 51 in Salvendy, 1997):

(1) the choice of which physical dimensions to use to represent values to-be-displayed, (2) maintaining the expected mapping of data values to levels of code dimensions, and 
(3) maintaining a correspondence between the direction of movement in the display and the direction of movement expected by the operator.

In the case where a spatial metaphor used in an interface is direct, as it is in 3-D displays that represent physical entities that vary in 3-D position and orientation, we must consider whether there are any physical dimensions that are already associated with the analogue concept to be presented. Roscoe (1968; and Roscoe et al., 1981) refers to this type of direct display as one which fulfils the "principle of pictorial realism". The principle of pictorial realism is an assertion that a display should present a spatial analogue of the real world, one in which the position of an object is perceived consistently and convincingly in depth as well as up-down and left-right. The principle encompasses many factors, including displaying information in an analogue format; maintaining a compatibility between object orientation and ordering that is consistent with the operator's mental model; and maintaining similarities in shape, direction of movement, and number of parts with the real world relative to the operator or controller. Pictorially realistic displays allow the operator to use a highly learned set of rules about the world to reduce the amount of information that must be processed before generating control responses. There is obviously a close tie between the concepts of pictorial realism and compatibility for dynamic analogue displays. It would seem that, within these concepts, guaranteeing proper compatibility between the code and its associated mapping should ensure the compatibility of movement. This is not always the case, however, as will be explained in section 3.3.2. 


\subsubsection{The Role of Teleproprioception and Telefinesis in Remote Control}

Recall, teleoperators are machines that extend "a person's sensing and/or manipulating capability to a location remote from that person" (Sheridan, 1994). A teleoperation system includes: devices for sensing the environment, devices to impart forces on the environment, a platform to move these devices within the environment, as well as the communication channels to and from the human operator and equipment in the remote environment. It is the presentation of sensed or modelled information about the environment and the teleoperator that, if designed correctly, provides some degree of telepresence, and thereby allows for reliable control of the telemanipulator for completing required tasks in the remote environment. Some of this information may be of a proprioceptive or teleproprioceptive nature, that is, information which aids in the human operator's sensing and keeping track of the location and orientation of the teleoperator and its appendages relative to its base, to each other, to external objects, and to the operator's own body if necessary. (Sheridan, 1994)

One type of teleproprioception is that which is naturally perceived and preserved by maintaining congruency between the control and display interfaces. This sense of proprioception is lost, or increasingly degrades, whenever the displayed information is sub-optimal, that is, substantially displaced from the mapped alignment defined by the optimal control-display relationship. The algorithm described in this thesis is an aid designed to reduce the number of errors and the time to completion of remotely performed tasks via many types of interfaces, be they of virtual reality, mixed reality, or 
real (video, infrared, $x$-ray, laser-range data, etc.) content. The information and its meaningful relationship to the task/control space are preserved via the algorithm.

In some cases, teleproprioception may be attained via viewing a computer-modelled virtual environment that reflects the happenings at the remote site (Sheridan, 1994). When such models are available, computer aids can provide a synthesised view of a teleoperator from any position and orientation chosen by either the human operator or the viewing system. In other words, in a virtual environment the viewpoint is not restricted by where physical video cameras may be positioned, but may roam the world under system or operator control in order to obtain any vantage-point deemed optimal or necessary.

Studies in the past have compared performance measures using different methods of providing views for computer-simulated telemanipulation. One such study is that of Das (1989, as cited by Sheridan, 1994) where he performed experiments within the context of using a computer simulation of a telerobotic task space to guide a snake-like telerobot between obstacles to reach a goal point safely. He systematically compared four different viewpoint selection techniques: free selection by the human operator, a view fixed just above a manipulator base (i.e. the typical egocentric view from inside the vehicle), a view fixed in space to one side of the task space and some distance away, and another view point determined by a "best view" algorithm. Das' results indicated that, in terms of speed and obstacle avoidance, performance was best when the operator was allowed to change the view freely, followed by the fixed point to the task and the automatic view 
selection. The worst performance was observed in the trials involving the "out the window" or egocentric viewpoint. Thus, the most effective teleproprioception, as expected, was achieved via operator controlled viewpoints. In Das' study, these results, and the fact that the egocentric viewpoint generated the worst performance, are important to note.

Closely related to proprioception is the idea that the brain can simulate movement by mental rotation, without actually observing it visually or haptically (Droulez et al., 1985 and 1986, as cited by Berthoz, 1991). The relationship between keeping track of present poses and predicting future locations and orientations of a controlled body is obviously the same relationship that ties the concepts of proprioception and mental transformation as they relate to telemanipulation. Internal simulation of a scenario permits the prediction of future positions and potential movements from those positions. A selection of possible actions for accomplishing the sensorimotor task from these, possibly redundant, potential solutions may then be made. The more accurately information about a situation is portrayed, the smaller the number of possible valid solutions that will be processed in evaluating the task execution. This reduction in the effective number degrees of freedom associated with the task space results in the alleviation of some of the load on mental processes and, thus, a decrease in task execution time.

The process of choosing a solution will depend on both the selection of possible configurations of expected sensory input available, and the human operator's ability to actively mentally manipulate reference frames with respect to the end goal. In other 
words, the path by which the operator comes to a decision about how he will execute the task is a function of the number of possible ways to do it and his ability to "see" that path in terms of mentally transforming and switching between the required reference frames. The idea of reference frames has been extensively discussed in the writings of psychologists. A distinction has been made between egocentric and allo- or exocentric frames of reference and their involvement in task execution. Berthoz (1991) indicates that the human brain is capable of operating in both frames of reference (e.g. induced perception of self motion, i.e. egocentric, and perception of a moving world, i.e. exocentric). The decision processing that takes place during task execution is accelerated by displays that support teleproprioception, mental transformations, and switching between ego- and exocentric frames of reference.

While proprioception is an awareness of posture and body-position, kinesthesis is a sense of motion. Telekinesthesis is also critical in terms of system feedback because, just as it is easy for an operator to lose track of the relative position and orientation of remote limbs and extensions, it is just as easy to lose track of how quickly they are moving and in what direction. The current means of mediating such confusion include using multiple views, a wide field of view from a nominal vantage point, and/or computer generated imaging. The first two solutions do not take into account the time varying nature of most teleoperation systems, however; that is, the informational requirements of the operator frequently change during the task and thus fixed viewpoints will not always be sufficient for adequate operation. 
The concept of congruency as discussed above refers to one-to-one correspondence between the display and the controls. When this exists, a control action will result in a corresponding change in the display with respect to the other control and display variables, resulting in movement in the same direction. When control-display congnuency is preserved it is then easier to maintain a sense of teleproprioception. Teleproprioception and a one-to-one correspondence between the displays and controls are required to keep track of the overall system dynamics, i.e. telekinesthesis. Designing with display-control congruency in mind is fundamental in human-factors engineering. Research elsewhere has determined, for example, that a $90^{\circ}$ or greater rotation between controller movement and displayed movements of the controlled object (which may be lagged) results in significant deterioration of tracking and manipulation performance (Bemotat, 1970). Other reports indicate tracking deficiencies which occur between $120^{\circ}$ and $180^{\circ}$ (Ellis et al., 1991; Macedo et al, 1997, and 1998). Cunningham and Pavel (cited by Sheridan, 1994) have shown that it is possible to reduce biases caused by displaycontrol rotations by providing proper cueing. In their study they were able to reduce the aiming error caused by a $108^{\circ}$ rotation by 70 percent via a "wind indicator" cue. The present study will attempt to show that mediating the rotation ${ }^{3}$, as opposed to identifying it explicitly, can more effectively reduce biases caused by dynamic display-control rotations.

\subsubsection{Proprioception and Sensori-Motor Control}

Roll, Roll and Velay (1991) proposed that spatially oriented motor behaviour is based on a body reference frame, which is determined by the concurrent processing of various 
sources of sensory feedback, which arise in the muscles that are stretched together in movement. These motor behaviours include a range of movements and body postures from head movements to pedal control. Typically in telemanipulation it is gross movement of the head, arm and hand that are of most interest. Roll et al. also proposed that this type of kinaesthetic body representation may be used as well in the spatial coding of purely retinal information in terms of egocentric co-ordinates.

When using non-isometric control devices (that is, devices which involve some degree of displacement), joint receptors, muscle spindles and cutaneous receptors in the skin around the joints and Golgi tendon organs may contribute to the proprioception of hand action (Zhai, 1995). Elastic devices furthermore allow both movement and force feedback through elastic elements, thereby eliciting responses from many proprioceptors, thus adding to the advantages of self-zeroing. Zhai suggested that such proprioception may not only improve static control accuracy but may also improve the dynamic aspects of control performance. Conversely, however, the effects of proprioception may amplify the disadvantages of any incongruencies present in the control-display relationship, thereby supporting the need to maintain control-display alignment. The innate advantages of proprioception thus illustrate why the principles of compatibility of display orientation and movement are important in control system design.

These principles of movement compatibility are associated with both current and expected mental representations of the user. Motor-oriented approaches to spatial cognitive reasoning assume that the principal metric for coding spatial relationships 
derives from the body's own movements in space. As explained below, Paillard (1991)

assumes that a sensorimotor mode of processing spatial information coexists with a representational mode and that both modes generate and store their own mapping of space:

"The sensorimotor mode concerns mainly that part of the physical world to which the organism is atmuned by virtue of its basic sensorimotor apparatus. Local sensorimotor instruments entertain direct dialogues with that world and thus contribute to the continuous updating of a body-centred mapping of extra-corporal space where objects are located and to which actions are directed.

The representational mode derives from neural activities which explore and consult internal representations of the physical environment, that are embodied in memory stores. They include mental representations of local maps, spatial relationships of routes relative to landmarks, relative positions between objects, and the position of the body itself in relation to its stationary environmental frame." (Paillard, 1991)

Paillard also indicates that visual information and proprioception co-vary and are used to support and calibrate each other. This shows that a mismatch between information from proprioception and that from the visual system has the potential to destroy any mapping of spatial relationships.

\footnotetext{
2 The term "teleoperator" is used here in its traditional sense: " a machine [or mechanism] that extends a person's sensing and/or manipulating capability to a location remote from that person", as defined by Sheridan (1994).

${ }^{3}$ Within the context of mental rotation here, mediation refers (operationally) to reduction of the need for mental transformation and the relaxation of the requirement to determine the direction of rotation before applying a control input to the system. The "wind indicator" cue in the previously described experiment indicated the direction of the required input explicitly. In this present study it is proposed that reducing the complexity of determining direction altogether may be a more effective means of eliminating control biases associated with performing mental rotations.
}

\subsection{Mental Transformations}

Mental transformations are costly when they complicate the execution of a teleoperation task. Control-display incongruencies "encrypt" display information and mental transformations are required to "decrypt" the information so that it can be used 
effectively in a control situation. However, these mental transformations load mental resources and the resulting excess mental workload is a source of time lag and error in task execution. The following sections describe spatial reasoning, the relationship between mental rotation and the upward direction, and the complexity of mental rotation in three dimensions. The section on spatial reasoning covers the topics of mental representation and manipulation of visual information. This is followed by a discussion of how the upward direction in most displays maps to the forward direction and how this relates to two-dimensional mental rotations. The final section describes the theoretical mental strategies used to deal with the mental rotation of objects in three dimensions. Teleoperation interface designers should keep these topics in mind when developing displays. The current study investigates teleoperation scenarios with both planar and perspective views of the task space, and thus the human characteristics that govern mental transformations in both two- and three-dimensional space are very relevant within the context of these experiments.

\subsection{Spatial Reasoning}

Whenever visual information has been sensed and the perception of objects continues, the visual or spatial information is mentally manipulated based upon the requirements of the particular task (Majchrzak et al., 1987). An important part of cognitive psychological research has been to understand the spatial representation of visual information and how that information is used to make decisions, solve problems and, more generally, to reason. Spatial representations can be identified as descriptions that characterise threedimensional entities or collections of entities, whereas spatial reasoning can be defined 
as the ability to make inferences based on the information contained within a spatial representation (Perricone, 1983, as cited by Majchrzak et al., 1987). Figure rotation is considered one particular type of spatial reasoning.

There are varying theories of mental rotation and the underlying mental processes that are involved in its performance (Shepard and Metzler, 1971; Cooper and Podgorny, 1976; Majchrzak et al., 1987; Yuille and Steiger, 1982; Barfield and Salvendy, 1985). The imagery or analogue model theory states that people can represent visual information in memory through images, which bear a 1:1 correspondence to the physical representation of the object. The propositional theory states that visual information in memory is transformed into propositions, where a proposition is the smallest unit of knowledge that can stand as a separate assertion. The two theories make different predictions about how visual information is internally manipulated. The first theory makes an implicit assumption that figure complexity does not affect the time required to mentally rotate the image, whereas in the second theory the number of propositions increases with complexity and thus the time to mentally rotate the image increases in direct proportion. Choosing to accept one of these theories affects the hypothetical complexity of the representation chosen for a teleoperator interface. However, as summarised below, regardless of which theory is correct, both endeavour to explain the fact that mentally rotating an object takes time, which is somehow proportional to the magnitude of the rotation angle, if not the complexity of the image. 
Shepard and Metzler's study (1971) indicated that participants mentally rotate an image first and then decide on a visual basis whether the given and target images are the same. Their critical finding was that the latency required to make a "same" judgement is a linear function of the angular disparity between the two images. This allowed for the speed of mental rotation to be estimated. Yuille and Steiger (1982) performed an experiment with figures similar to those used by Shepard and Metzler, in an attempt to study the effect of object complexity. They found that people likely use different strategies to perform mental rotations based on the complexity of the figure. If the figure is fairly simple, they will use all the features to solve the task. If the figure is fairly complex, too much information must be temporarily retained in memory and the person must reduce the number of components attended to or perform poorly. In fact, their study revealed that angular disparity had less of an effect with complex figures than with simpler figures. They claimed that the alternative piecemeal strategy applied to more complex figures is less demanding than applying the holistic rotation to simple figures.

Barfield and Salvendy (1985) performed an experiment similar to Yuille and Steiger's experiments, but asked subjects: if the displayed figure was rotated in depth or in the picture plane, whether the figure was rotated in $x-, y-$, or $z-a x e s$, and to indicate the angle of rotation. Their findings revealed that complexity of the figures had effects on axes of rotation and the angle of rotation but not on the depth/picture plane question. For all three questions, the required rotation had statistically significant effects on the time required for performing the task. 
Those results thus further indicated that performance does not fit neatly into one of the theoretical frameworks to the exclusion of the other. Instead, a hybrid model for the mental rotation task appears to be the most appropriate to explain the data. For simple levels of image complexity people mentally rotate the figure in an analogue manner into congruence with the stored version of the memorised test figure. As image complexity increases, people seem to perform the task by a feature-by-feature analysis. Subjects' verbal accounts in Barfield and Salvendy's study indicated that they did in fact use this hybrid strategy. Furthermore, the strategy implemented may have an effect on the errors made. Working on individual features in memory means that the integrity of the object is destroyed and the features may be placed back together incorrectly. For simple figures the integrity of the object could be maintained and the features would remain in the same relationships after mental transformations.

In summary, the above findings indicate that mentally rotating any object involves mental transformations, that take time regardless of the question being asked. Thus, a display that eliminates or reduces the requirement for mental rotation should promote a decrease in task completion time and a decrease in the frequency of control errors.

\subsubsection{Mental Rotation and the Forward Direction}

Early experiments on mental rotation required participants to compare the shape of test patterns presented in different orientations relative to each other (Shepard and Metzler, 1971) or to their own canonically defined orientation (Cooper, 1975; Cooper and

Shepard, 1973). Since the subjects' egocentric frame was aligned with the fixed 
environmental frame, they generally imagined a rotation of the localised objects relative to their fixed environmental or, equivalently, egocentric frame of reference. Cooper and Shepard (1973) then performed an experiment in which advance information about the orientation of the object to be presented was provided, and found that there was evidence of preparatory mental rotation only if they also provided advance information about the identity of the ensuing stimulus. This suggests that the participants in Cooper and Shepard's experiments prepared for a specific visual shape in a specific orientation by imagining the shape rotated into that orientation even before it was presented to them. On the other hand, it was difficult for them to prepare for an unknown shape given only an abstract frame to mentally rotate in preparation for interpreting the test stimulus.

Cunningham and Pavel (1991) have examined a related case of mental transformation that tested the strength of the natural mapping of the visual "up" to the motor space forward direction and humans' ability to adapt to unseen visual-motor transformations that change this relationship. Their experiments reveal that transformations between visual axes and motor axes can lead to undetected biases (i.e., not consciously realised by the operator) in an operator's input to a system. These biases may not be detected when evaluating overall performance because some transformations affect only a subset of the operator's input. This means that, even when a visual-motor transformation is present in the system and operators are aware of it, they may still not realise that their performance is being adversely affected if they monitor only their overall performance. This evidence suggests that the natural mapping of the visual "up" to the motor space forward direction is strong. 
Shepard and Hurwitz (1984) state that the concept of an upward direction has subsequently been extended to represent horizontal directions that are (1) uphill, regardless of gradient, (2) upward, in the metaphorical sense of toward a salient or important reference object or location, (3) northward, in the global environment frame, or (4) straight ahead, in the viewer's egocentric frame. Their investigation of the latter coupling between the upward and straight-ahead directions involved the interpretation of bends in a line. The study revealed that interpretation time increased with angular departure from upright of the line going into the turn (in the context of path following), suggesting that this type of task does require a mental rotation. Shepard and Hurwitz's reasoning behind the relation between the upward and forward directions is summarised in the following paragraphs.

Since our standard, conventional viewpoint is somewhat elevated above the generally horizontal surface of the ground upon which we live, two points on a path leading away in front of us project onto an intervening vertical plane (and thus onto the retina) with the farther point interpreted as above the nearer point. This concept is illustrated in Figure 2. Correspondingly, in visually fixating and/or pointing first to the closest point (A) and then to the farther point (B) on the horizontal path ahead, we must move our eyes and/or arm upward. In standard pictorial representations there is thus a natural correspondence between the forward direction in the horizontal plane, which is orthogonal to a gravitationally defined upright, and the upward direction aligned with that upright. This makes it clear why people prefer to rotate maps so that the upward direction corresponds 
to the direction of travel since, in doing so, there is a direct agreement between left and right turns on the map and within the environment.

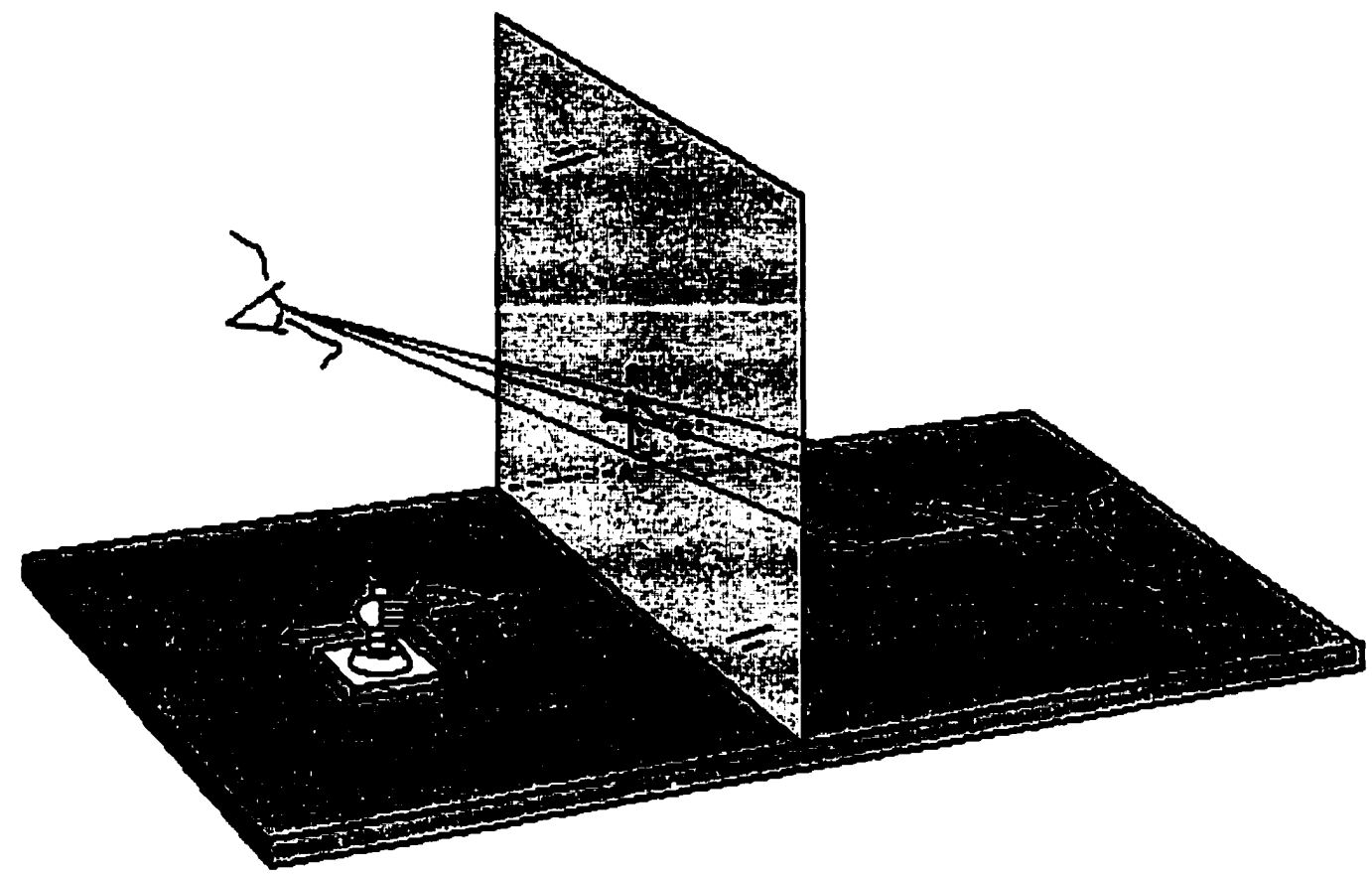

Figure 2 The correspondence between the standard viewpoint perception of "ahead or forward" and the "upward direction" (adapted from Shepard and Hurwitz, 1984)

There is, however, a high cognitive cost associated with substantial departures from this preferred alignment, as has been documented by Levine and his co-workers in his study of "you-are-here" maps (as cited by Shepard and Hurwitz, 1984). That particular study revealed that, when the preferred alignment was used to present such maps, people who consulted them were usually successful in finding their way directly to their destination. However, when the map was inverted, people who consulted the map went off in directions that were at least $90^{\circ}$ in error more than $25 \%$ of the time. The mental mechanisms which are in part responsible for this finding may also be responsible for the 
execution of control reversals whenever a remotely controlled vehicle is not aligned with the orientation of the controller device. (This is a static mapping problem. The dynamic "principle of moving parts" may also partially explain the occurrence of control reversals, as will be discussed in section 3.3.4.)

In addition, Shepard and Hurwitz (1984) also conducted a continuous, steady state "map reading" experiment that simultaneously tested the relative importance of both departure from upright and departure from the orientation relative to the preceding turn. Their investigation, which may possibly be extended in relevance to vehicle control, involved the presentation of three connected lines on a display representing a path of travel (see Figure 3). The thin line (\#3) represented the path just travelled, a slightly thicker line (\#2) connected to the thin line represented the path currently being travelled, and a third even thicker line connected to the opposite end of the current path line represented the path to be turned on. In other words, the departure of the second line from the orientation of the first thin line was similar to the current position of a controlled object and the following (thickest) leg of the turn's departure from upright represented the target or un-travelled path to which the controlled object must move. Results of Shepard and Hurwitz's 1984 study of a "continuous map-reading task" indicated statistical reliability of "the departure from upright of the most recently added segment, and the ... agreement or non-agreement between the absolute direction of the last added segment ... and the participant's classification of the turn into that last segment as left or right." The responses became faster when there was an agreement between the absolute direction of the upcoming turn and the response - which consisted of turning a switch to either the left or to the right. 
(This suggests the operation of an overall stimulus-response-compatibility effect that we would also expect to see in the results of the current study.)

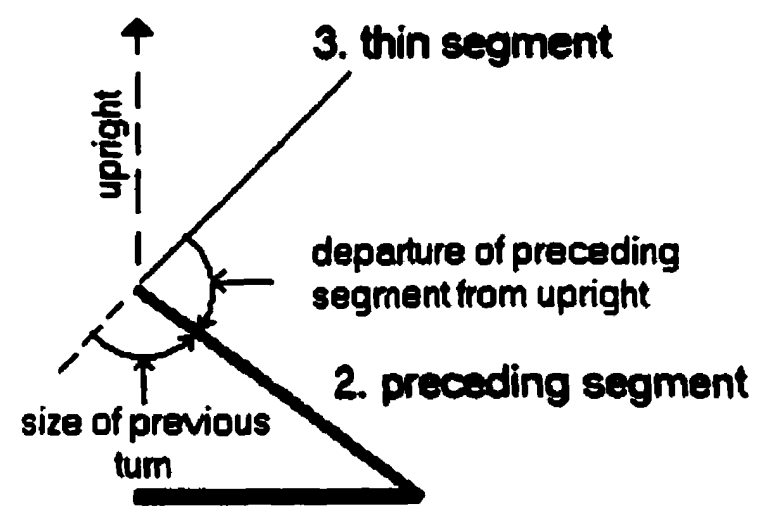

1. last added thick segment

Figure 3 An illustrative example of an experiment from as found in Shepard and Hurwitz's chapter, 1984

Reversal errors are the result of misinterpreting an instrument's indication and making a control movement that aggravates rather than corrects the undesirable condition (Johnson and Roscoe, 1972). The related use of the term 'up' helps humans to find their way or communicate about the physical environment. In this particular case, 'up' is determined by one's momentary egocentric frame; in terms of one's physical being which may easily be transformed into correspondence with the presentation of one's avatar in a visual display.

Two frames of reference are of interest here. The first is one's own egocentric frame, which is defined by the directions of up-down, front-back, and left-right with respect to one's own body. The second is an object-centred frame, similarly defined with respect to some other person, animal, or object (e.g. a vehicle) on the basis of its own visible 
properties defining top-bottom, front-back, and left-right sides. People in general can successfully discriminate between enantiomorphic ${ }^{4}$ versions of simple objects (e.g., normal versus backward alphanumeric characters) or between subtle perturbations of leamed shapes, if an object is presented in an unexpected orientation. A person seemingly makes this discrimination after imagining the canonical upright representation rotated into the orientation of the presented object (Cooper, 1975; Cooper and Podgorny, 1976; Cooper and Shepard 1973). In fact, it has been hypothesised that such mental rotation is required even for the most familiar of shapes. For example, Cooper and Shepard (1975) have realised that people can readily recognise and identify if a picture of a hand presents fingers in an upward or downward direction, and/or back or palm out, regardless of its orientation. However, if the portrayal is not in its canonical orientation the determination of whether the presentation is of a left or right hand seems to require a mental transformation.

\subsubsection{Mental Rotation in 3 Dimensions}

As cited above, Shepard, Roscoe and their colleagues have researched mental rotation both in two-dimensional space and with two-dimensional depictions of three-dimensional objects. Huggins and Getty (1982), on the other hand, have investigated some of the difficulties human operators are likely to encounter in viewing and using abstract, volumetric, three-dimensional displays in practical applications. They operationally define the term "abstract" display as one in which the image is constructed, as opposed to being reproduced veridically, as in a TV image; and a "volumetric" display as one in which the (virtual) image viewed is space-filling - that is, where various points on an object image are at different depths from the observer. They achieved this by using a 
vibrating mirror display. Their study investigated how the speed and accuracy of responses in a choice reaction task were degraded as the orientation of the stimulus image varied relative to that of the fixed response array. In addition, the effects of symmetry in the shape and position of the cue used to indicate the orientation of the displayed object to the operator were investigated:

Displays such as that studied by Huggins and Getty are typically used to present information about the relative position, orientation, and movement of vehicles in remote control situations, in order to permit operators to make control decisions. Their study investigated the speed and accuracy of pose identification of an object presented in an arbitrary orientation, and involved rotation of a marked cube about each of its three axes. The response time functions fall into three general categories: fast, flat functions showing no effect due to rotation; peak-shaped functions in which the response time increased almost linearly with rotation away from the head-on orientation; and plateau-shaped functions that began like the peak-shaped functions, but indicated no increase in response time beyond rotations of 90 degrees relative to the head-on orientation. Three strategies were suggested to explain these functions, respectively. The Spatial strategy is the fastest and easiest strategy, and depends on a direct mapping from the displayed object to the control object. This strategy can therefore be applied only when the displayed object appears in a head-on ${ }^{5}$ orientation or when distinguishing marks lie on the axis of rotation.

A Relational strategy is also used, depending upon the presence of an asymmetry of the displayed object to mark the object's orientation and to distinguish between an otherwise 
confusable set of orientations. Lastly, a Rotational strategy, in which the observer mentally rotates his viewpoint to a position from which the cube would appear in a headon orientation, may be used followed by an application of the Spatial strategy.

The results of Huggins' and Getty's study demonstrated that the Spatial strategy is the fastest and most efficient when conditions allow its application. The Rotational strategy is more efficient than the Relational strategy when the object is near to the head-on orientation, that is, when the amount of mental rotation is small; however, when the rotation increases beyond 90 degrees this strategy rapidly becomes less efficient. When the Spatial and Relational strategies cannot be applied operators are forced to use the slower, less efficient Rotational strategy.

Control-display incompatibility can be reduced by the appropriate coding of the orientation of objects within the display. It is also very important that any object presented in a 3D display be asymmetric. This would permit operators to determine orientation directly from the displayed object without having to resort to the potentially slow and inaccurate rotational strategy. These results suggest that, in traditional remote vehicle control or other similar displays where fast, accurate responses are at a premium, an inside-out (or egocentric) view, or a view providing comparable informational attributes, should be provided to the operator. As the angular discrepancy between the displayed view (the outside-in view) and controller orientation (which is congruent with the inside-out view) increases, reaching its maximum when the operator views the controlled object head-on (in the traditional sense, as it applies to teleoperation, as 
opposed to from behind as in the Huggins and Getty study, 1982) rather than from behind, having this type of viewpoint becomes increasingly important. The importance of an egocentric or ego-like viewpoint when control-display discrepancies become large has also been considered by Roscoe (1968) in his comparison of typical canonical "northup" versus "heading-up" map presentations for navigation, although the discrepancies discussed here are much larger than those in that study.

\footnotetext{
"Enantiomorphic refers to the relationship between structures that are mirror images of one another.

S In the Huggins and Getty, 1982, study the object of interest in the experiment is a cube where the canonical front of the cube was defined as the marked side facing the subject and the right side of the cube was the side facing the subject's right in this orientation. Thus, the spatial strategy in this experiment applies when the cube is in the "headon" orientation. This is not typical of teleoperation scenarios where the front of the object of interest normally faces away from the operator. In such cases the term "head-on" should be replaced with the term "forward facing"
}

\subsection{Navigation and Control}

Three of the critical tasks in the navigation of large scale spaces are judgement or awareness of where things are, control of movement, and checking, to ensure that the map - which portrays the goals of navigation - is congruent with the forward field of view (Wickens, 1999). Because each of these tasks contributes in its own way to the operator's mental workload (due to mental transformations, etc.), and thus to time delays and control errors, the human factors designer of navigational displays should strive to minimise this mental workload wherever possible. The challenge, in other words, is to understand the mental transformations that mediate the different reference frames, predict their cost, in terms of time, etrors, and workload, and understand how any potentially adverse affects may be attenuated by display design or by improved cognitive strategies. Another challenge is to optimise the frame of reference for presenting navigational information, to support the various tasks that must be accomplished. 


\subsection{Siruational Awareness}

Endsley (cited by Wickens and Carswell, in chapter 4 of Salvendy, 1997) defines situational awareness as "perception of the elements of the environment within a volume of time and space, the comprehension of their meaning and the projection of their status in the near future." One of the critical factors in maintaining situational awareness is the operator's limited working memory. It is the integration of information in working memory with information about earlier phases of the task, along with the operator's general knowledge of system functioning (his or her mental model), that determines comprehension of the current situation, and prediction of the future.

Global situational awareness is one of the reasons why an exocentric view is used in many teleoperation tasks. In Das's experiments (as cited by Sheridan, 1994, and reviewed here in Section 3.1.1), the egocentric viewpoint resulted in the worst performance in a computer-simulated telemanipulation task. On the other hand, although an exocentric viewpoint is better in terms of providing information that supports situational awareness, it can also result in incompatibilities between the display and the ego-referenced controls, as discussed above. It is incompatibilities such as these which can further load the dynamic working memory, resulting in potentially longer completion times and greater frequencies of error. 


\subsubsection{Spatial Compatibility}

Compatibility between a display and its associated control has two components. The first component relates the location of the control relative to the display and the second to how the display reflects (or commands) the control movement. The location of the controls and the direction of control movements both have similar impact in this study. The compatibility principle of congruence, with respect to location of controls, states that the spatial arrangement of a set of two or more displays should be congruent with the arrangement of their controls (Wickens and Carswell, chapter 4 in Salvendy, 1997). Secondly, with respect to orientation of controls, the spatial orientation of a display should be congruent with the arrangement of its controls. The distinction between "left" and "right" in designing for compatibility can be expressed relative to some prominent axis, in either relative terms or absolute terms. This axis may be the body midline, a prominent visual axis of symmetry in the system, or the sagittal plane separating front from back. Fitts and Posner (1967, cited by Wickens and Carswell, in Chapter 4 of Salvendy, 1997), have stated that, when left-right congruence is violated, the operator will have a tendency to activate the incorrect control, particularly in times of stress.

Compatibility between a display and its associated control also depends on how the display reflects the control movement. This aspect is defined as intention-responsestimulus (IRS) compatibility (Wickens, 1992). There are a set of rules which govern how the operator's intention to respond maps to the actual response, and then to the display signal. People in general have a conception of how a quantity or entity is ordered in 
space. It is therefore beneficial to design control and display movement to be consistent with the form and direction of this ordering.

Of particular interest to the present study is compatibility between displays and rotary controls. Operators have certain stereotypical expectations about how the movement of a rotary control will be associated with the corresponding movement of a display. These expectations have been classified as stereotypes of proximity of movement and stereotypes corresponding to congruence (Wickens, 1992). The proximity of movement stereotype states that, with any rotary control, the arc of the rotating control element closest to the moving element of the display is assumed to move in the same direction as that display element. Some examples of this stereotype in real applications are illustrated in Figure 4. The case that is shown in part (c) of Figure 4 is similar to that encountered using rotary control devices for any rotating display element, be it in 2 or 3 dimensions. The display and controls in the figure have no associated front or back and thus tend to be more compatible with each other than those that do (e.g. a joystick). That is, in considering the dial in Figure 4 (a), which has no markings, one may note that the dial may be left in any position without violating the principle of congruence or the principle of movement proximity. However, with a pivoting joystick, which unlike the dial does have a front and a back, violation of the two principles is possible, just as the dial violates the principle of movement proximity in Figure 4 (c).

This problem is highly relevant to the field of telemanipulation because many controls and displays share this type of "front-back" relationship. The front of the telemanipulator 
may be associated with the front of the controller, as in the case of an anthropomorphically designed controller, the forward/upward facing part of the operator's hand or arm may form the matching half of the control mapping. This component of movement compatibility relates to congruence (Wickens and Carswell, chapter 4 in Salvendy, 1997). Displays and controls should move in a congruent fashion: linear controls parallel to linear displays and rotary controls congruent with rotary displays. The aforementioned rotary control-display pairings, such as that depicted in Figure 4(c), are compatible in terms of congruence but violate the proximity of movement stereotype in certain situations.

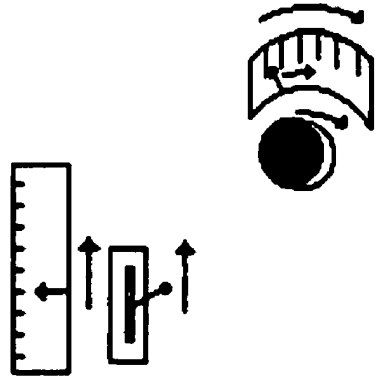

(a)

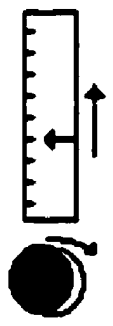

(b)
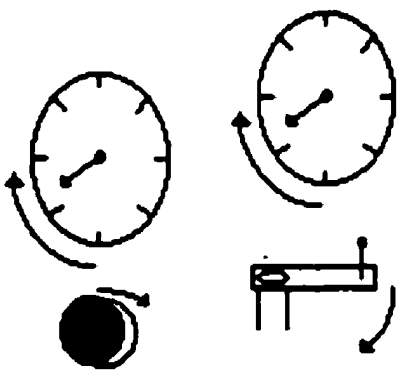

(c)

Figure 4 Some population stereotypes in control display relations (adapted from Wickens' figure in chapter 4 of Salvendy, 1997)

The principle of control-display compatibility is one of the fundamental principles in human factors. It is the violation of this basic principle that leads to one of the most serious classes of problems in teleoperation (cf. Figure 4(c) as discussed above). In designing controls and graphical aids for tele-control and tele-navigation it is important to maintain correspondence with three (of the many) principles of compatibility which 
together describe control-display congruence. These principles are (Wickens and Carswell, chapter 4 in Salvendy, 1997):

(1) the principle of movement compatibility - the indicator of a display should move in the same direction as its control,

(2) the principle of compatibility of display orientation - the orientation and spatial ordering of a display should be consistent with those of its associated mental representation, and

(3) the principle of compatibility of display movement - the direction of movement of the moving part of the display should be consistent with user expectations.

It is sometimes most easy to design while keeping a fourth principle in mind: the principle of conceptual compatibility, which asserts that the layout and operational methods of controls should be consistent with user expectations or population stereotypes. However, if two stereotypes conflict, or if obeying the principle of conceptual compatibility in a design violates one of the three aforementioned principles, then serious problems can arise.

As an example of compatibility of display movement, and how this may end up conflicting with other principles, there is a large body of experimental and operational evidence that states that the part of the display that represents the object being controlled directly should move against a fixed scale or fixed co-ordinate system, rather than having the scale move against a fixed index representing the controlled system (Fogel, 1959; 
Poulton, 1974; Roscoe, 1968; Roscoe et al., 1981). The explanation for this is simple; when an operator moves a control, he naturally expects the display indication resulting from that control movement to move in the same direction. Furthermore, when the operator moves a control he presumably knows what he is controlling, and that is not the world relative to the controlled system, but the controlled system itself. Thus, he expects the symbolic representation of the vehicle to respond to his input.

The "proper" direction of motion of a display with respect to the outside world may best be considered within the context of figure and ground (Johnson and Roscoe, 1972; Roscoe et al., 1981). Psychologically, the part of a dynamic field of view that is perceived to be stationary is traditionally called the background, or ground, and the object that is perceived to be moving is called the figure (or cursor). When the entire visual field moves in relation to the observer's eyes, the observer may perceive selfmotion ${ }^{6}$ against a stationary background (Fitts and Jones, 1961, as cited by Roscoe et al., 1981). In relation to Roscoe's aviation research, as illustrated in Figure 1, the question then becomes whether apparent motion relationships between the aircraft (cursor) and the outside world (background) are retained when the pilot's attention is focused on a small attitude indicator or other display, inside the vehicle's cockpit. When highly resolved, dynamic, literal ${ }^{7}$ images are used, stable figure-ground relationships are retained, and control reversals are rare (Roscoe et al., 1966 as cited by Roscoe et al., 1981; Roscoe, Johnson, and Williges, 1980). However, when the operator views a small, abstract instrument representation of the outside world (a cue-depleted representation), other 
factors, such as the instrument control panel or the framed aperture of the individual display face, can cause a figure-ground reversal (Johnson and Roscoe, 1972).

The principles of control-display motion compatibility suggest that the moving element in the display should be the aeroplane and that its movement should be in the same direction as its controls. Control reversals with a moving horizon display are usually associated with a sudden response to a temporarily unnoticed change in attitude (Johnson and Roscoe, 1972). In other words, when changes in aeroplane attitude are subtle and pilot attention is not focussed on the attitude-direction indicator, it is more likely that a control reversal will occur at this time than another. However, during routine manoeuvres, with the pilot's full attention, no trouble was encountered. This suggests that the attitude display might be improved by permitting the aeroplane symbol to move in the same direction as the controls for fast responses. The slower responses could then continue to be displayed as per the moving horizon paradigm. These suggestions lead to the creation of the frequency separation concept that was first introduced in Figure 1 of Chapter 2 (and which will be discussed in Chapter 1 in greater detail).

\subsubsection{Spatial Awareness, Navigation, and Control}

Navigating through environments, both real and virtual, relies on the resources associated with Logie's "visuospatial scratchpad"8 (1995 cited by Wickens and Carswell, chapter 4 in Salvendy, 1997). Although we are able to navigate through many worlds using some combination of route, survey and/or landmark knowledge, there are many situations in which we require navigational aids (Wickens, 1992). Such aids may take many forms, all 
facilitating guidance to a target using varying degrees of pictorial realism. Electronic and paper maps are two types of such aids. While electronic maps can vary in terms of the extent to which they rotate in the direction of travel, both paper and electronic maps can vary in terms of representing a planar or perspective view of the world. In using such navigational tools to gain spatial awareness of an environment, the navigator must first establish the extent of congruence between the two. In other words, the navigator must answer two questions, one regarding where he is in terms of location and orientation, and the other being to what extent does this position correspond with where he wants to be. Determining the extent of congruence may involve a number of different mental transformations that add both time and effort to the navigational task (Aretz and Wickens, 1992; Wickens et al., 1996).

Figure 5 illustrates two remote control helicopter scenarios in which cognitive transformations play a key role. The monitor in the top-left comer contains the forwardlooking field of view (the inset within window (d)) from within a remotely controlled helicopter. The bottom-left figure depicts the same scene presented in the form of a north-up plan view map. The map-view (c) in the upper right corner illustrates the $180^{\circ}$ mental rotation that the human operator must perform in order to create congruence between the map (a) and the remote camera view (d). In addition, it also illustrates the $90^{\circ}$ mental rotation required to create congruence between the map (i.e. its mental representation in the track up orientation) and the forward-looking field of view to facilitate local navigation (i.e. a $90^{\circ}$ rotation from (a) through (b) to the track-up orientation). Note that in order to achieve complete congruence with the "out-the- 
window" displays (d) or the control in the bottom right corner the map displays (c) and the track-up display between (b) and (c) must undergo an additional $90^{\circ}$ rotation into the horizontal plane. These types of information transformation are time consuming, effortful, and a potential source of both navigational and control errors.

Studies have been performed in which rotating maps were investigated with respect to how they altered the mental workload associated with such mental transformations (Aretz, 1991; Wickens et al., 1996). This was achieved by rotating the maps presented to the navigator into correspondence with a track-up orientation, thereby eliminating the need for a mental rotation and attempting to facilitate navigation. Haskell and Wickens, and Wickens and Prevett (1993 and 1995, as cited by Wickens and Carswell, in chapter 4 of Salvendy, 1997) have performed further navigational research in which guidance information is presented in a 3-D format. They showed that, if one were given a forward looking view into an environment and 3-D navigational cues (such as colour coding, etc.) as aids, the requirement for mental transformation would be reduced and navigational performance would improve considerably. The benefits are enhanced even further if the viewpoint of the navigational display has the same zoom factor as that which would be perceived by the avatar, looking forward, rather than a viewpoint that is from behind and outside the teleoperated system. 


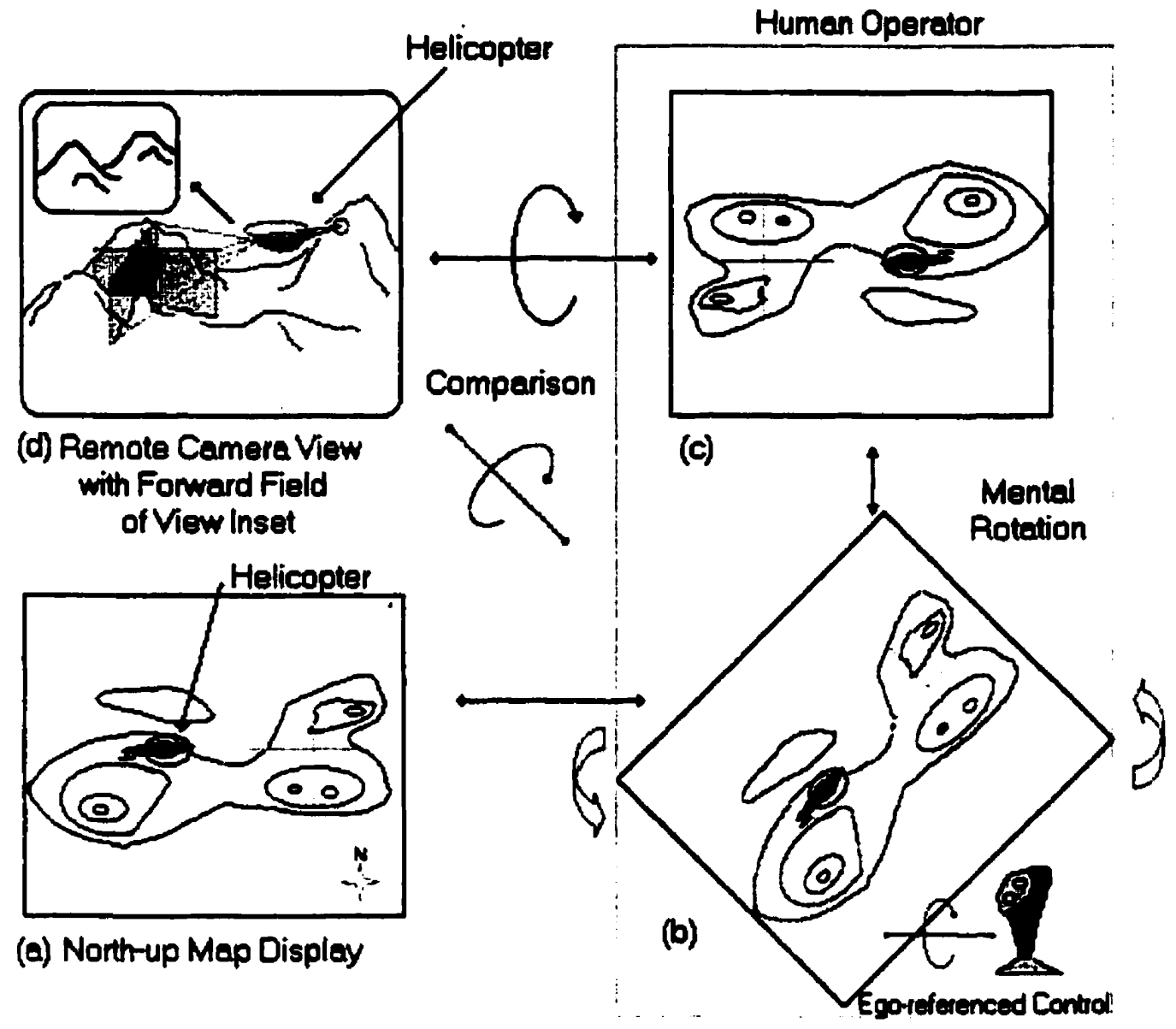

Figure 5 Remote control helicopter example (concept adapted from Wickens, 1992)

Mental rotations required to integrate:

- a world-referenced north-up map display (a).

- the same image in an exocentric "remote camera" view fixed to the side of the task space (d) which provides global situational awareness about the real world,

- an ego-referenced forward field of view (i.e. the inset in the upper left comer of (d)) which provides information required for local guidance,

- an ego-referenced control device (lower right comer of the figure next to frame (b).

- Mental transformation from (a) the world-referenced north-up map to the egocentric inset in (d) follows the path (a) $\rightarrow$ (b) $\rightarrow$ (d). Mental transformation from (a) the worldreferenced north-up map to (d) the exocentric view follows the path $($ a) $\rightarrow($ b $) \rightarrow(c) \rightarrow(d)$.

Such displays may lose their advantageous properties, however, should the navigator suddenly find himself lost, disoriented, or required to make a spontaneous departure from 
the planned route. This is the double-edged nature of the navigational display: those features that make a navigational display best for guidance, will diminish its effectiveness in supporting the spatial situational awareness that is necessary for successful recovery from a state of geographical disorientation. This is likely one of the reasons that the egocentric viewpoint in Das's experiment (c.f. section 3.1.2) resulted in the worst performance in the telemanipulation task.

Figure 6 illustrates the parallel between a centricity continuum and the views that support local guidance and global awareness. The view to the far left is an egocentric view, from within a car, that supports local guidance with its out-the-window properties. The view to the extreme right of the figure is that which is associated with an exocentric viewpoint of the same car. This viewpoint supports global awareness. The viewpoint in the centre has characteristics of both the egocentric display and the exocentric display. This viewpoint is connected to the car by means of a "tether". The tether causes the view to move with changes in the car's egoposition while at the same time the view is removed from the nominal viewpoint within the car's driver seat, permitting the operator to view additional exocentric details. Thus, in a very general sense, the "tethered" viewpoint supports both local guidance and global awareness. The tether concept is discussed in greater detail in section 3.4 . 


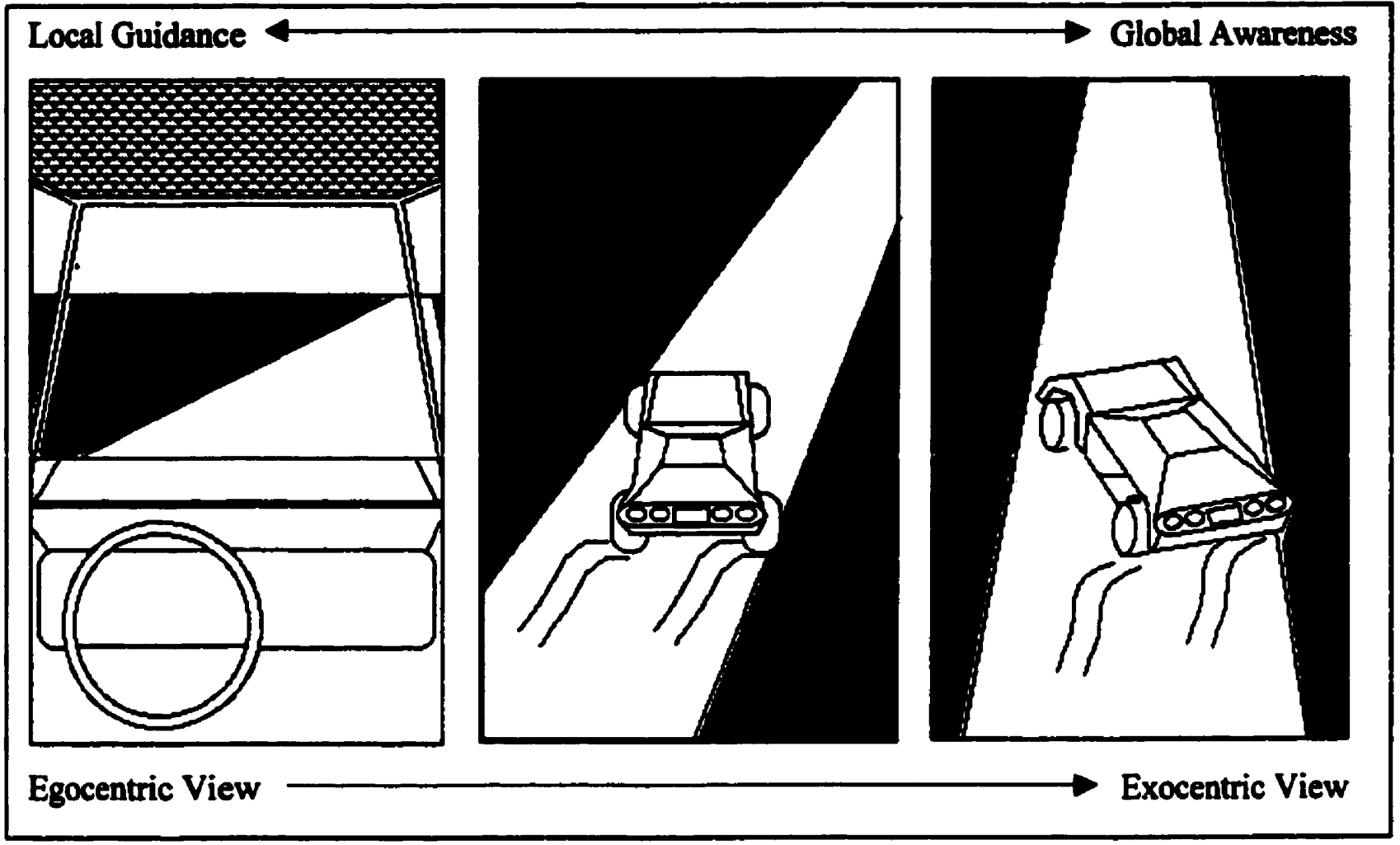

Figure 6 Illustration of egocentric, tethered, and exocentric display viewpoints and their relationship within a continuum spanning local guidance to global awareness.

Figure 7, which parallels Figure 6, simply illustrates explicitly the proposition that the level of exo-centricity of a display increases with the distance between the object of interest's nominal viewpoint (the driver's seat in Figure 6) and the location of the given viewpoint.

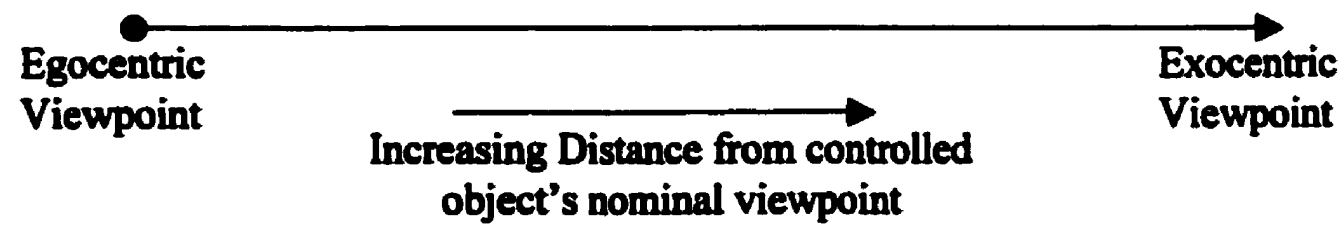

Figure 7 Illustration of centricity continuum concept 


\subsubsection{The Effect of Incongruency on Navigation}

The expectations derived from the relationships shown in Figure 2 and the empirical results from Levine et al (1982, as cited by Shepard and Hurwitz, 1984), reflect the fact that people generally report that it is easier to interpret a turn as a right or a left turn when the road that leads into that turn heads upward on a map. Whenever a road is followed in the downward direction, all relationships are reversed. Many people then find it easier to turn the map upside-down in order to restore the simpler relations. If the map is left in its canonical orientation, typically north upwards, then one must use mental transformations to compensate for any large departures from the upright direction. If the direction is in the vicinity of $180^{\circ}$ from the canonical direction and the turns are at approximately $90^{\circ}$, then a simple strategy of reversal may be adopted, i.e. a turn to the left is simply a turn to the right, and vice versa.

Such a strategy often fails to yield correct results for arbitrary approach angles and arbitrary turn angles, however (Shepard and Hurwitz, 1984). The more reliable strategy of mental rotation is used in such cases, but to do so for an entire map at once would most likely exceed the capabilities of visual memory. A number of alternative strategies which may be used in such cases have been proposed:

(1) Imagining each successive turn separately rotated from its given orientation into a more interpretable canonical orientation, in which the leading leg of the turn is pointing upward, allowing an easier determination of the proper direction of the turn to be made. 
(2) Imagining a schematic representation of a canonical right turn and rotating it appropriately for each new turn and applying a match/mismatch test.

(3) Performing (b), except in such a way that the rotation is performed from the last recalled position of the schema.

Strategies (a) and (b) are particularly applicable for vehicle control; however, the time to classify each should increase monotonically with its angular departure from upright (Cooper and Shepard, 1973, 1978; Shepard and Cooper, 1982). An analogy may be made by referring to the rotation and discrimination between a normal and backward ' $L$ '. It is apparent that applying such a strategy for successive turns would be very slow and inefficient, at which point strategy (c) may be used to perform path following and dynamic tracking tasks more effectively. Studies by Shepard and Hurwitz (1984) have shown that subjects are not likely to apply strategy (c) in a dynamic path following task.

As departure from the canonical position increases, we would expect to see an increased frequency of interpretation errors if an increase in response time is not prevalent. This is due to a speed-accuracy trade-off that governs direction interpretation. As the discrimination of direction becomes more difficult, more control reversals will be apparent. A control reversal is operationally defined here as a control movement in a direction opposite to that intended by the operator. Typically, a decrease in the number of control reversals will result in an increase in average response time, while an increase in the speed of the response will also increase the frequency of control reversals. Both measures are used to evaluate performance in the control experiments in this thesis. 
Aretz (1991) conducted an experiment comparing a map display employing the principle of visual momentum9 with the two traditional approaches, track-up and north-up. The data collected show that the track-up alignment is congruent with the ego-centred forward view (as illustrated in Figure 6 by the similarities between the middle pane, which may be likened to a track-up display from a perspective viewpoint, and the left pane). Local guidance is supported by the track-up display because of this congruence with the egocentred forward view. However, one of the cognitive tasks involved in pilot navigation is the development of a global cognitive map, and the track-up display is not well suited for this type of mental operation. Thus, the subjects in Aretz's experiment did not fully benefit from the rotating map because it did not allow them to construct cognitive maps of the task space.

It would be interesting to discover what the effects of using a frequency separated rotating map would be on such a task, in terms of its support of both local guidance and the construction of cognitive maps. Instead Aretz had chosen to implement an alternative ego-referencing tool via visual momentum. We can, however, obtain much relevant information from his study. Several comparisons can be made between Aretz's study and the one presented in this report.

(1) Aretz presents a theoretical framework that views navigational awareness as the maintenance of a cognitive coupling between two reference frames that correspond to a canonical map view and a forward view of the world. The present paper, 
analogously, presents a theoretical framework that views situational awareness as the maintenance of a cognitive coupling between reference frames that corresponds to information required for global awareness and that required for local guidance.

(2) To navigate, a pilot must be able to associate the current view of the world to its location on the map; in the present study, to safely teleoperate an operator must be able to associate the attitude and position of the teleoperator with his controls.

(3) The ego-centred reference frame (ERF) is established by a pilot's forward view out the cockpit window and directly corresponds to this perspective. The aircraft's heading provides the ERF's canonical axis. In our case the ERF is established by the operator's view of the world through the display - be it into the display for a forwardlooking perspective view or in the upward direction for a plan view of the remote location .

(4) A visually presented map with a canonical north-up alignment establishes the worldcentred reference frame (WRF). In our case there is no consistent WRF because the frequency-separation algorithm continuously shifts what might normally become a WRF map into alignment with the ERF.

Although the two investigations are very similar, they ask questions with two slightly different goals in mind: navigational awareness and situational awareness. Having reviewed the past literature on situational awareness and mental rotation, the present 
project proposes that an operator must mentally rotate the WRF into congruence with the ERF in order to obtain full awareness for local guidance in teleoperation. Two mental rotations are involved in this transformation (Aretz, 1991). These transformations are also illustrated in Figure 5. First a rotation is performed to bring the WRF (map view (a) in Figure 5) into a "track-up" alignment (a map view between that shown in (b) and (c) of Figure 5). This may be accomplished by either digitally or electronically rotating the displayed image, or by mentally rotating the image of the arbitrarily set viewpoint (which may be north-up or otherwise). Clearly such a rotation is not required if the viewpoint is already aligned with the track-up orientation. Second, a forward mental rotation is required in order to bring the track-up image around the horizontal axis and into direct alignment with a nominal forward viewpoint for the object being controlled and the operator's controls (the transition from (b) to the inset within view (d) in Figure 5). These rotations align the image completely with the ERF (represented by the view given by the inset in window (d) of Figure 5) and thus complete control-display congruence is achieved.

Aretz's paper delves extensively into the extension of mental rotation from visual objects to visual scenes and maps, and references several researchers who have provided empirical support, including the work of Eley, Evans and Pezdek, Hintzman, O'Dell, Amdt, Levine, Shepard and Hurwitz, and Sholl. The need for mental rotation is a part of the cognitive process that supports spatial/situational awareness and this need may be eliminated by providing track-up alignment in the design of plan or bird's-eye view teleoperation displays ${ }^{10}$.If the display is rotated such that the ERF and WRF are already 
aligned, then the operator need not perform this mental rotation and must only perform the remaining forward mental rotation. As already noted in prior sections, such an alignment simplifies control greatly, because all azimuth deviations become strictly left or right relative to the current orientation. Some studies have found that fewer errors are committed with track-up maps in spatial awareness tasks (Marshak, Kuperman, Ramsey, and Wilson, 1987; Rossano and Warren, 1989). Aretz also concludes that for tasks that primarily involve the ERF, such as that of localisation, the track-up alignment will result in the most effective performance.

The research reported here attempts to provide a solution that facilitates the maintenance of situational awareness (for local guidance and global awareness) by implementing a frequency-separation algorithm. This solution also takes advantage of the concept of visual momentum. Woods (1984, as cited by Aretz, 1991) describes the concept of visual momentum as the characteristic of a system design that influences an operator's ability to extract and integrate information across several displays. A high degree of visual momentum aids the operator in maintaining a cognitive representation of the system by presenting one display's information in the context of another or by making an effective smooth transition from one display to another. The goal of applying visual momentum is to provide perceptual landmarks, or anchors, that aid in the maintenance of a cognitive representation of the data. The use of visual momentum has been investigated, for example, in the design of plant process control/monitoring systems (Burns, 1998) and it has been successfully applied in the design of a hierarchical file system (Vicente, 1988). In the present work, the author suggests that visual momentum may also be applied 
effectively to teleoperation, and that frequency-separation can be used as the means to achieve this visual momentum, by allowing the operator to integrate information across an ERF and WRF during changes in the visual scene following operator control actions.

\subsubsection{The Effect of Incongruency on Control}

Another topic of study that concerns itself with the same issues reviewed above is threedimensional tracking with misalignment between display and control axes. Ellis et al. (1991) have performed extensive research on this topic, conducting two experiments, which examined three-dimensional pursuit tracking when operators of teleoperation simulations were faced with misalignment between the display and control frames of reference. Their experiments have shown that the worst tracking performance seems to be at misalignment angles greater than $90^{\circ}$, with peak errors occurring at approximately $125^{\circ}$.

As an explanation of these results, subjects' ability to compensate for rotations of the reference viewing grid with respect to the control axes of the joystick involves a mental rotation of the type described by Cooper and Shepard (1973). The mental rotation requires time that manifests itself as a control latency. This induces a lag between the target trajectory and the human response that is proportional to the azimuth angle used to

generate each display condition. Maximum tracking errors occur at a rotation angle greater than $90^{\circ}$ because, depending on how the operator visualises the mental rotation, at least some of the mental rotations will require a reversal of control, i.e., rotating the image into the $180^{\circ}$ alignment and implementing the simple direction reversal strategy. 
Typically operators will tend to choose mental rotations that do not require such reversals. Assuming that reversal of control will add additional time lags and further degrade tracking performance, especially for relatively rapid inputs, it is speculated that the operator will choose to make mental rotations greater than $90^{\circ}$ just to avoid performing the mental control reversal. There is thus an angle beyond which the delay caused by a mental control reversal is acceptable, because the alternative rotation (to $180^{\circ}$ ) becomes so small that the combined mental rotation and reversal times is faster than performing a rotation into the compatible orientation. For larger angles, the total delay becomes a decreasing function of azimuth; that is, as azimuth increases, the amount of the required mental rotation decreases.

Hintzman, O'Dell and Amdt (1981, as cited by Wickens, chapter 39 in Boff, Kaufman and Thomas, 1986) explored the use of mental rotation when people judge directions to surrounding objects or places after imagining themselves in a changed orientation. Their experiment involved showing subjects a target dot that could appear in any one of eight locations in a circle, with an arrow at its centre pointing to any one of these locations. As illustrated in Figure 8, subjects were asked to judge in which direction the arrow should be rotated in order to achieve alignment with the designated target dot by rotating a stylus on a response board to the appropriate position relative to a forward facing arrow. As with the results obtained by Cooper and Shepard (1973), and assuming that subjects mentally rotated the configuration of the arrow plus the target, reaction times for nearly all types of responses increased linearly with increasing angular departure from upright. There is an exception for the condition in which the arrow was rotated $180^{\circ}$ from the 
upright orientation, however. The decrease in response time in this condition may be attributed to subject selection of the reversal technique that allows for faster responses when the rotation angle is large.

There are basically two approaches that may be implemented to compensate for problems of misalignment with respect to control. One is to dynamically alter the effect that system input has on the display (Macedo et. al, 1997; Macedo et. al, 1998; and Tani and Sheridan, 1983), and the other is to dynamically alter the display relative to the control input. The study of the latter approach is the topic of the present report, while the study of the former approach is the topic of the Macedo et al. 1997 and 1998 publications. The objective of their study was to discover a method to automatically compensate for misalignments so that the human operator can achieve levels of performance comparable to aligned conditions without additional training requirements. Their algorithm for automated compensation resulted in significant improvements in operator performance. However, this type of solution may not always be feasible in every situation. 


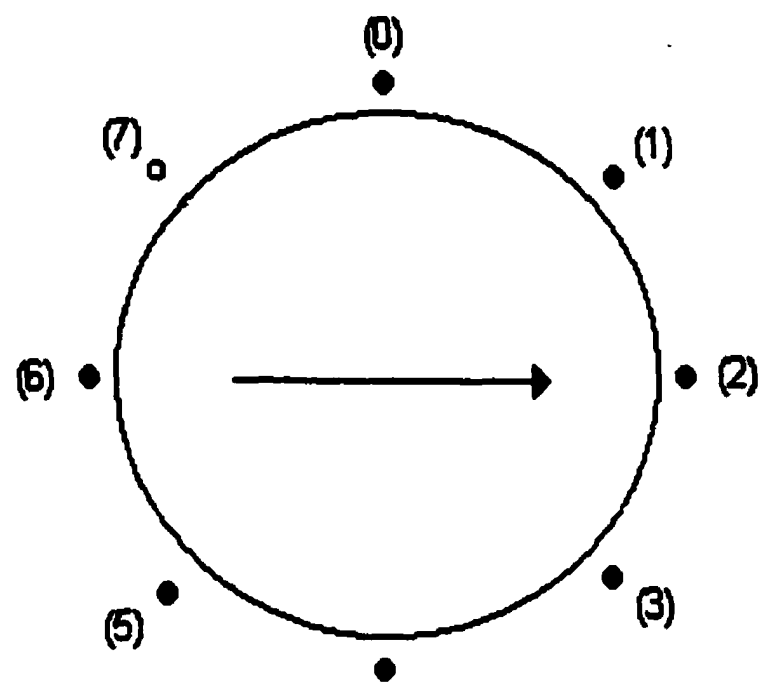

(4)

Figure 8 Experimental Display used by Hintzman, O'Dell, and Ardnt in 1981 to determine the role of mental rotation in determining direction (as cited by Wickens, chapter 39 in Boff, Kaufman and Thomas, 1986)

Macedo et al. cite Proctor and van Zandt (1994), who indicated that the orientation of an operator relative to the display screen also has varying effects on control performance in teleoperation, due to three factors: visual-motor compatibility, control-display compatibility, and visual-trunk compatibility. Non-optimal controller-display alignments exist in many situations, such as when a robot that is being manipulated is free to rotate and the operator is not, when the operator is off-axis, and when the operator is viewing through video cameras attached to the manipulator - such as in laproscopic surgery. In Macedo et al's study, peak errors occurred for controller-display rotations between $90^{\circ}$ and $270^{\circ}$.

\footnotetext{
' Aside: This finding is particularly interesting, because frequency-separated displays, which are described in later sections, can cause both the background and figure to be in motion simultaneously. Should the operator perceive this as illusory self-motion then the display may potentially induce disorientation and even motion sickness.

"The term "literal" refers to very accurate images.
} 
The visuospatial scratchpad exemplifies the notion that spatial working memory represents information in an analogue spatial form, often typical of visual images.

'Visual momentum is a concept that provides a solution to the problem of becoming cognitively lost as a user traverses through multiple displays pertaining to different aspects of the same system or database. It involves the following four basic guidelines: using consistent representations, using graceful transitions, highlighting anchors, and displaying continuous world maps (Wickens, 1992).

${ }_{10}$ However, in doing so, other issues such as violations of the principle of the moving part arise because the controlled object no longer moves relative to the stationary display frame. This is due to the fact that trackup displays qualify as a pursuit type II display which will be described in further detail in later sections.

\subsection{Frames of Reference}

Before starting our discussion about interface design concepts that merge these frames of reference, we must first clarify the meaning of some of the terms that we use here. Milgram and Colquhoun (1999) operationally define the term "centricity" to refer to the extent to which a human observer's viewpoint is removed from his "ownship", i.e., from the nominal viewpoint with respect to the viewer's own-avatar, own-vehicle, or ownmanipulator within the teleoperation environment (in accordance with Figure 6 and Figure 7 as discussed earlier). This concept is illustrated explicitly in Figure 9, where various camera viewpoints relative to a remotely controlled excavator are depicted. The continuum at the bottom of the figure expresses how the degree of exocentricity increases with the increased displacement of the viewpoint from the nominal viewpoint. The nominal viewpoint relative to the vehicle in Figure 9 is from the cab looking out the front windshield, as shown at the far left. (Recall that this view is similar to that described as the ERF of the aeroplane discussed by Aretz, 1991, c.f. section 3.3.4.) In the figure the nominal viewpoint is illustrated as the excavator cab to the far left over the "egocentric" extreme of the centricity continuum. 
Across the top of Figure 9 are two similar but non-parallel continua. The first is the reference frame continuum, which illustrates the close relationship between the egocentric view and a view that is classified as Ego-referenced. At the other extreme, a viewpoint that is not fixed to the task space but to the world is classified within the World-referenced frame. However, unlike the centricity continuum, which continue to become more exocentric as distance from the nominal viewpoint increases, the reference frame continuum ends at this point, since world-referencing is independent of viewpoint distance from the nominal viewpoint. In other words, with respect to the referencing axis, world referencing commences as soon as the (virtual) camera is detached from the system being controlled. The second, "awareness" continuum, across the top, is more like the centricity continuum in that global awareness increases with increased exocentricity, but it also increases with changes along a secondary dimension -- the number of orthogonal viewpoints. As the number of viewpoints increase so does global awareness (although not necessarily local navigation). At the opposite end of the continuum, on the other hand, the integrated egocentric view supports local navigation within the task space.

Different viewpoint characteristics or frames of reference, are required for the two classes of situational awareness that are required in most teleoperation scenarios. Frames of reference (FORs) for presenting navigation and control information to support various tasks may be defined by various viewpoints relative to the operator's momentary field of view. Various FOR locations are illustrated in Figure 9. These show a decrease in egocentricity and an increase in world referencing as we move from left to right along the continuum. This figure reiterates the infinite extension of the centricity continuum 
concept in the exocentric direction introduced in Figure 7 and illustrates the line of thinking that ties ego- and exocentric viewpoints together in a meaningful manner.

Wickens (1998) classified a variety of viewpoints for displays that define various frames of reference. His discussion is focussed on the domain of 3D aviation displays. Nevertheless, the same concepts may be extended to cover a wide range of applications. Wickens describes two types of relationships that define the interaction between the viewpoint (e.g. camera) and ownship (e.g. the avatar). These relationships are couplings in terms of orientation and position. The orientation may be fixed with respect to the heading of the ownship. Wickens recognises this relationship as one which is "heading slaved". This type of coupling usually makes sense only when the position of the viewpoint is fixed with respect to the position of the ownship. This is called a position slaved or "tethered" display (Faye and Wickens, 1995; Wickens, 1998), as illustrated in Figure 9. Tethering causes displayed information to move with ego-position, such that momentary direction of travel and ownship are depicted at a constant size. Now, if the orientation of ownship is uncoupled from the viewpoint, the display is said to be "heading fixed". It then follows that the uncoupling of position is called "position fixing". Both of these describe world-referenced relationships given to a viewpoint. An example of a heading fixed viewpoint is the display of a north-up map.

The centricity continuum also encompasses an idea developed in a paper by Wickens (1998) - the rigid tether. The tether combines the information formats presented by both egocentric and exocentric viewpoints. The egocentric viewpoint causes the visual display 
to change with changes in ego-position, perceived as egomotion -- a view also known as "inside-out". In contrast, the "outside-in" view, or exocentric view, fixed in a world reference frame does not change with ego-position, but permits observation of the movement of ownship while increasing global spatial awareness about ownship. Tethering combines the local guidance benefits (e.g. display scaling or scrolling) of maintaining alignment with ego-motion while providing a sense of greater spatial awareness due to the extemally referenced viewpoint.

Table 1 Situational needs of the Teleoperator (adapted from Blackwood et. al, 1997, as cited in Milgram and Colquhoun (1999b)).

\begin{tabular}{|c|c|}
\hline & $\begin{array}{l}\text { Global Situational Awareness } \\
\text { Navigation information } \\
\text { Location of avatar (or ownship) } \\
\text { Location/movement of other system entities }\end{array}$ \\
\hline & $\begin{array}{l}\text { Local Guidancessituational Awareness } \\
\text { Target identification and location } \\
\text { Terrain / object distance estimation } \\
\text { Cueing presence of relevant objects (in field } \\
\text { of view) }\end{array}$ \\
\hline
\end{tabular}

The two extremes of the continuum in Figure 9 correspond to two different reference frames and similarly two different kinds of knowledge which are required to perform tasks within each frame. It is important to distinguish between operations which require information in support of local task execution and those which require support for global awareness. A well recognised summary of how some of those information needs contrast with each other is presented in Table 1. Ego-referenced knowledge is expressed in body-relative terms and supports local guidance or local situational awareness. Worldreferenced knowledge is expressed within a world-referenced co-ordinate frame and supports global hazard (situational) awareness. A navigator can relate ego- and world- 
referenced displays by performing a series of mental transformations, which however impose costs in time, mental effort, and possible errors (Wickens, 1995; Faye and Wickens, 1995, Milgram and Colquhoun, 1999a).

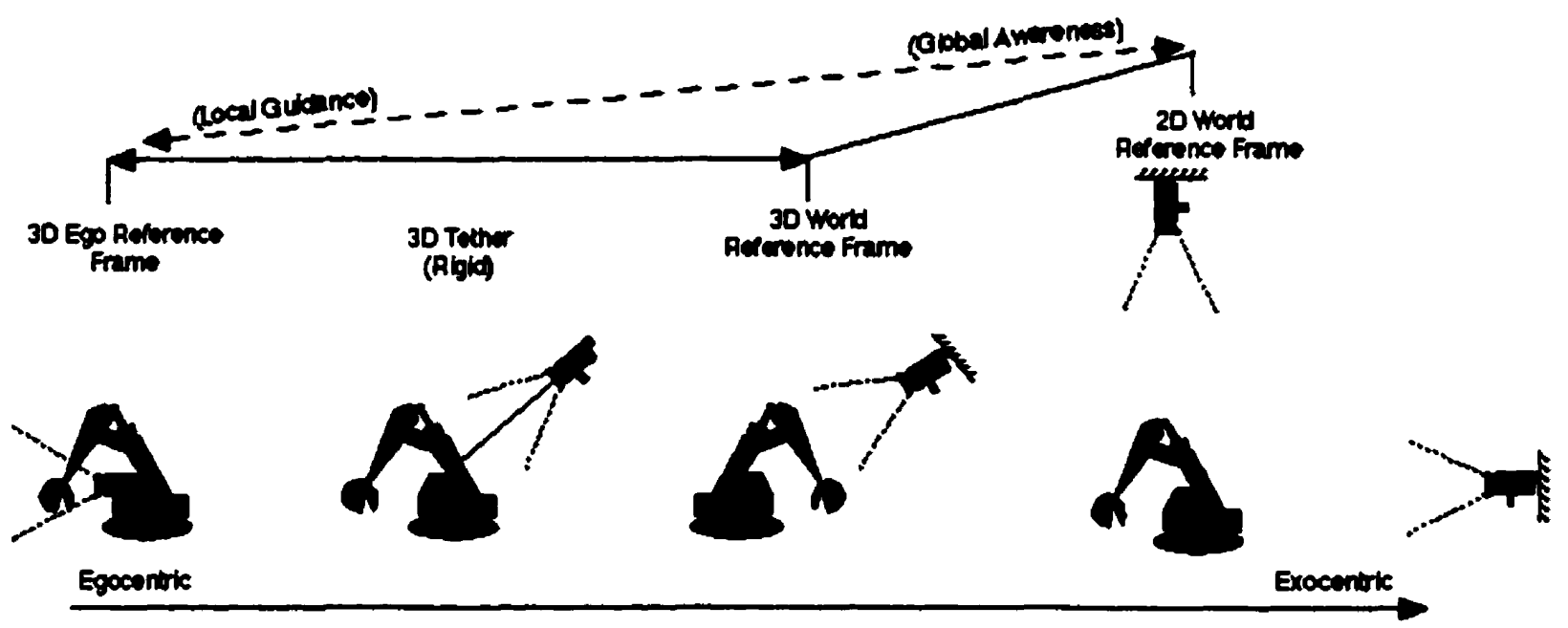

Figure 9 Centricity Continuum: Illustration of the transition from ego- to exocentric viewpoints (from Milgram and Colquhoun, 1999, adapted from Wickens, 1995)

Wickens (1995) also states that rotating maps appear to provide an advantage in terms of all measures of local guidance and that this advantage appears to more than offset the lesser, intermittently observed advantage of the north-up maps for the retention of terrain features in spatial memory. He and others (Olmos, Wickens, and Chudy, 1997; Wickens 1995) also found that the egocentric "immersed" display is superior to any of the two (3D or 2D) extremely exocentric displays for guidance tasks and navigational checking, but was inferior to them in terms of providing global awareness. This was due to the "keyhole" view of the world from within ownship (c.f. Das' study in Sec. 3.1.2). This contention provides further encouragement that a mixture of the characteristics of the two display types through the vehicle of tethering is promising. 
When maps are fixed in their azimuth angle, rather than rotating with the direction of travel (as do immersed and tethered track-up displays), local navigational and thus control decisions are challenged by spatial incompatibility, whenever the direction of travel is away from the canonical orientation (Wickens, 1999). This phenomenon is well established in navigational tasks of all kinds, including: aviation, where actual navigation in locomotion is required (Wickens, Liang, Prevett and Olmos, 1996; Olmos, Liang, and Wickens, 1997; Aretz, 1991); surgery (Flach, 1999; Milgram and Colquhoun, 1999b); in pure mental rotation, where discrete directional decisions are required (Shepard and Metzler, 1971; Shepard and Cooper, 1973; Hajchrzak et al., 1987; Yuille and Steiger, 1982; Barfeild and Salvendy, 1985); and when image comparison for navigational checking is required. Spatial incompatibility causes mental transformations to be performed that would not be required of an immersed or tethered display. These transformations can lead to increased time of task completion, as well as an increased likelihood of error.

Two explanations have been offered for the cost of "azimuth angle incongruity" (Wickens, 1999). These reasons may be viewed as either alternative explanations or as possible additive or interactive effects; further research must be performed to determine which is the case. The first explanation assumes that this cost reflects the same kind of linear mental rotation as that studied in object rotation tasks (e.g. Shepard and Cooper, 1982; Shepard and Metzler, 1988). However, in this case the mental rotation defines viewpoint rotation. The second explanation is that the cost represents a symbolic 
transformation or reversal mapping. Accordingly, we might expect the cost to be nonlinear with azimuth rotation; i.e., small when rotation is less than $90^{\circ}$ and distinctly larger when it is greater than $90^{\circ}$, at which time mappings must be reversed. Indeed such a pattern can be found in the map rotation data collected by Aretz (1991). Support for this non-linear view is also provided when comparing the different azimuth angles by which the viewpoint is tethered behind ownship. Across experiments we find little effect for this azimuth offset until at least $45^{\circ}$ (Wickens and Prevett, 1995; Wickens et al., 1996).

The immersed ego-referenced display does not adequately support geographical awareness because of the "keyhole effect" (Woods, 1984, as cited by Wickens, 1999), illustrated in the far left panel of Figure 6. With this view only a small portion of the world can be viewed at a time, and any search through $360^{\circ}$ space may be awkward, nonsystematic and unnatural. Such displays have been found to be poorly suited for providing global knowledge of where other objects are in relation to one another (McCormick et al, 1998). The exocentric viewpoints support that aspect of situation awareness related to searching and finding relevant entities.

Tethering facilitates the viewing of a greater region of the geographic space surrounding ownship, as does a fisheye view; both of which may be implemented as an attempt to better support situation awareness. Problems have been cited with both of these possible solutions, however. The tethered view creates a 3D ambiguity in that the length of the vector between ownship and any other target is somewhat ambiguous and may have a deleterious effect on task performance. On the other hand, expanding the geometric field 
of view with the fisheye lens technique distorts the depicted direction of all objects, except those directly viewed along the line-of-sight. Wickens' (1999) research indicates that the distortion caused by the fisheye view is more harmful to the accurate location of objects than is the ambiguity caused by the tethering technique. In general, egoreferenced situational awareness questions are better answered by a rotating egoreferenced frame, and world-referenced questions are better supported by fixed maps.

In conclusion, we have seen in this section that many teleoperation tasks demand visualmotor skills that require a high level of training and experience. Such situations demand a man-machine interface that provides a manageable level of operator workload. Efficient correspondence between controls and display instrumentation are critical in reducing operator workload. 


\section{Pursuit versus Compensatory displays}

This chapter discusses the differences between pursuit and compensatory displays and describes the advantages and disadvantages of each with respect to tracking performance. Then the notion of mixing the displays to capture the advantages of both while using the characteristics of each to offset the disadvantage of the other is introduced and the possible implications of such a design are detailed. A discussion of how the order of the control system and the frequency content of the tracked system factor into the design of this mixed display for optimal tracking concludes this section.

\subsection{Distinctions between compensatory and pursuit displays}

Two basic ways to present etror information on a tracking display are pursuit and compensatory displays, as illustrated in Figure 10. Pursuit displays (also known as "following" or "true-motion" displays) have at most two moving indices, one representing the desired position or course (i.e. a target marker) and the other the actual position (i.e. a cursor) (Poulton, 1974). Both symbols move against a common scale or background reference system. A compensatory (or "relative motion") display normally has only one moving index. The direction and distance of this moving index relative to a fixed (world-based) reference represents the error to be nulled. As explained below, in this mode, target and controlled-system movements are completely confounded. That is, the source of the input driving the moving index can not be determined solely by visual observation because its motion is the combined result of both system disturbances and control input. Research comparing the two display types has, for the most part, shown pursuit displays to be superior to compensatory display presentations (Poulton, 1974). 
This problem associated with compensatory displays is the cause of frequent errors due to operators' misunderstanding of the display. The principal advantage of a pursuit display over a compensatory display is that it shows explicitly the nature of an error signal. In other words, pursuit displays show not just simply what the track is doing and what the output of the controller is, but they have the added advantage of informing the operator, through these two signals, about how the error arose.

In 1950 and 1952, Poulton (1974) showed that compensatory displays give an average modulus mean error about one third larger than pursuit displays. He also showed that the superiority of pursuit displays became more pronounced as the level of task complexity increased (i.e. by increasing the complexity of the target track). In fact, at the highest levels of task complexity, subjects would have produced lower error scores by doing nothing rather than attempting to control the system when using the compensatory display. This is due to the fact that compensatory tracking obscures the effects of an operator's own control inputs by mixing them with the target's motion. Nevertheless, in certain situations compensatory displays do have some advantages over pursuit presentation. These will be further discussed later in this study. 


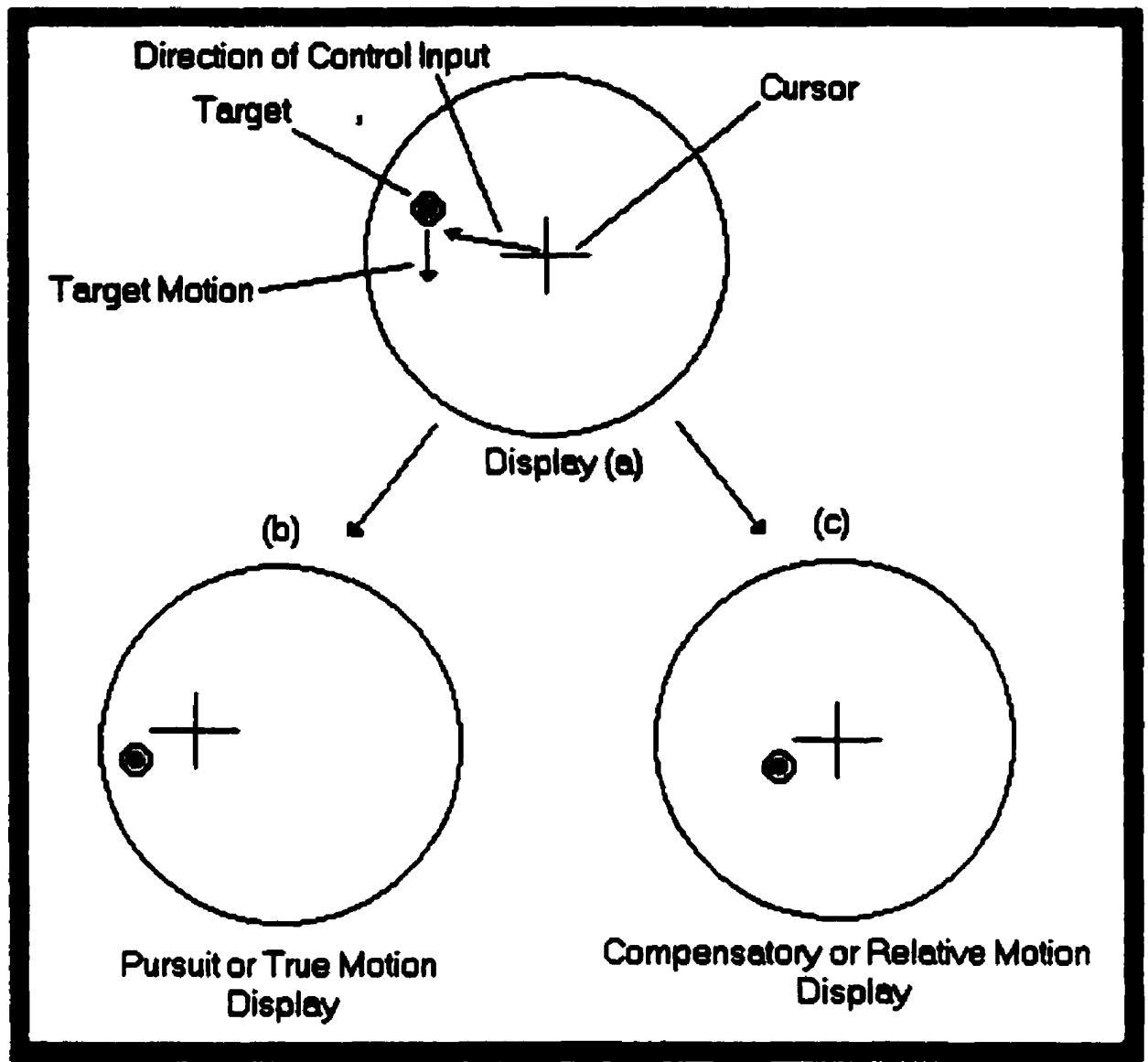

Figure 10 Illustration of the distinction between pursuit (true motion) and compensatory (relative motion) displays: Display (a) becomes display (b) at a later time when the display format shows the motion of both the target and the cursor, as well as the error (i.e. the distance between the two markers). Alternatively, display (a) becomes display (c) when the display format permits only motion of the target marker, thereby conveying only the change in the system error.

The tethering technique described in Chapter 3 (see Figure 9) can also be looked upon as defining a pursuit-compensatory display continuum. Referring to Figure 10, an extremely flexible tether (the limit being zero rigidity) would result in little or no camera movement in relation to movements of the cursor (i.e. the object being controlled). The resulting display characteristics in this case would be identical to that of a pursuit-type display, since both cursor (the excavator in Figure 9) and target (the goal of the control 
action) movements may be observed against a stationary background, and thus the relative position of one with respect to the other would be easily perceived.

A very rigid tether, on the other hand, would result in $100 \%$-coupled motion between the "virtual camera" and the cursor. In this case, the resulting display characteristics would be similar to that of a compensatory-type display, since only the error between the cursor and the target would be observed directly, while any other information would have to be inferred. In addition, the only real motion perceived would be the apparent motion of the target relative to the cursor; i.e., the change in error between the two objects as the view of the world changes in response to control actions. Perception of cursor motion relative to the world frame would thus be somewhat degraded due to the lack of relative motion between the cursor and the camera.

It is important to note, however, that such rigid tether displays postulated in the latter case can arguably be considered not to be true compensatory displays, since the continued presence of a background can still provide the observer the means to infer individual cursor and/or target motions indirectly (via the phenomena of induced motion). Nevertheless, although cursor and target marker motions may be derived, the resulting background motion information is not usually in the proper format for immediate and accurate interpretation of either marker's movements. This is due to the lack of controldisplay motion compatibility, discussed earlier in this report. It is for this reason that, in spite of the existence of arguments to the contrary, the author considers this type of "rigid tether" display to be more similar to a compensatory display than a pursuit display. In 
other words, the presence of a background may not significantly improve task performance over the case of a traditional compensatory display, for which only the error is visible and there is no backdrop information available. Further research is needed, however, to compare the two.

Summarising the above discussion, as the tether connecting the camera and the avatar becomes more rigid, the advantages of the pursuit display are diminished and thus, the display becomes more 'compensatory-like', where the error signal is the primary, and possibly the only, visible and useful information. The cursor/avatar thus becomes more like a zero marker (as in the case of a cursor-referenced compensatory display, as illustrated, for example, on the right side of Figure 12) and therefore the relative motion between the cursor and target becomes confounded to a greater degree and thus less discriminable by direct human perception. Even though the presence of a backdrop can theoretically provide much of the information conveyed by an equivalent traditional pursuit display, the format of this information is likely to be unintuitive.

\subsection{Mixed Displays}

If varying the rigidity of a viewpoint tether defines a pursuit-compensatory continuum, then the intermediate points on that continuum define pursuit-compensatory hybrid displays with varying "proportions" of pursuit and compensatory characteristics. Pursuitcompensatory hybrids have been studied by others, including Jensen (1979, cited by Roscoe et al., 1981) and Senders and Cruzen (1952, cited by Roscoe et al., 1981). Early work of Jensen described two ways of combining pursuit and compensatory indications 
in a single display (as reported in Roscoe, Corl, and Jensen, 1981). One was by distance of movement proportions and the other by frequency separation of the vehicle's motion. In either case, some fraction of one symbol's motion was removed and placed on the other symbol, with the effect being less deviation of one symbol from the reference point. As illustrated in Figure 11, another option considered was to mix pursuit information, cursor-referenced compensatory information, and target-referenced compensatory information in a percent-of-distance manner. This mix of the three components may be specified by positioning a point anywhere on the perimeter of the triangle shown in Figure 12, indicating the percentage of pursuit versus compensatory in the vertical direction and the percentage of cursor- or target-referencing in the horizontal direction. The continuum between pursuit and compensatory displays is described in this study indicated by the label "FSD" in Figure 12. However, for a detailed discussion of the triangle and the continuum between the cursor-referenced and target-referenced the reader is referred to Roscoe, Corl, and Jensen's 1981 paper entitled "Flight Display Dynamics Revisited".

In a similar manner, Senders and Cruzen (1952, as cited in Roscoe et al.,1972 and 1981) studied tracking performance with what they called "combined compensatory and pursuit tasks". The output of a subject's control was in series with the fraction of the problem signal that drove the cursor and was not in any way related to the motion of the target. Only the problem generator (or some complementary fraction of the problem signal) moved the target. Thus, their study involved target-referenced compensatory displays. The pursuit display consisted of two display markers (error and track) which moved 
together when the error between them was zero, and the compensatory display consisted of the same two display markers that remained stationary together when the error between them was zero.

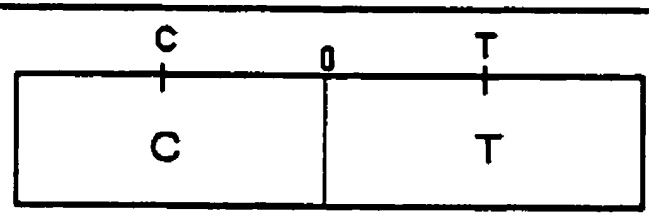

(a) Pursuit displey

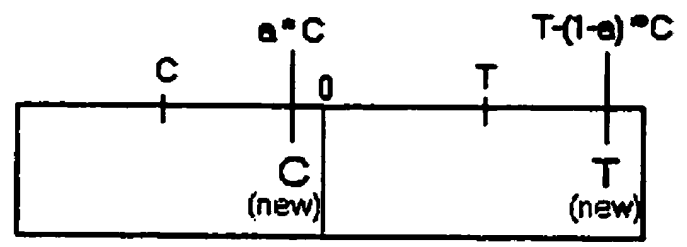

(b) Cursorreferenced compensettory displey $(a=1 / 6)$

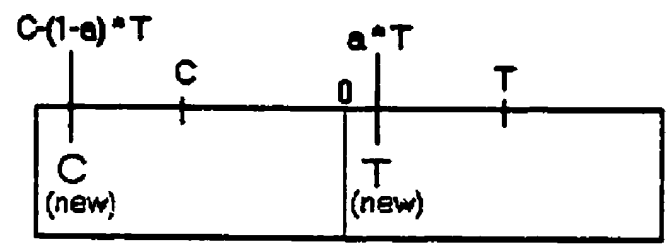

(c) Target-reterenced compensatory displey $(a=1 / 6)$
Figure 11 Functional schematic of pursuit, cursor-referenced compensatory and targetreferenced compensatory displays: (a) the true positions of the cursor, $\mathrm{C}$, and the target, $T$, are shown relative to a zero null position, (b) cursor-referenced compensatory displays place the cursor at a fraction, e.g. $a=1 / 6$, of the true distance while the target is placed at its true distance less any distance the cursor is not displaced, e.g. $(1-1 / 6)^{*} C$, and (c) a targetreferenced compensatory display places the target marker, $T$, at a fraction of its true distance and the cursor marker is placed at its true distance less the remaining true distance of the target. Note that all three cases shown here present the same situation; that is, the distances between $C$ (new) and $T$ (new) are the same in all three cases. (Adapted from Roscoe et al., 1981).

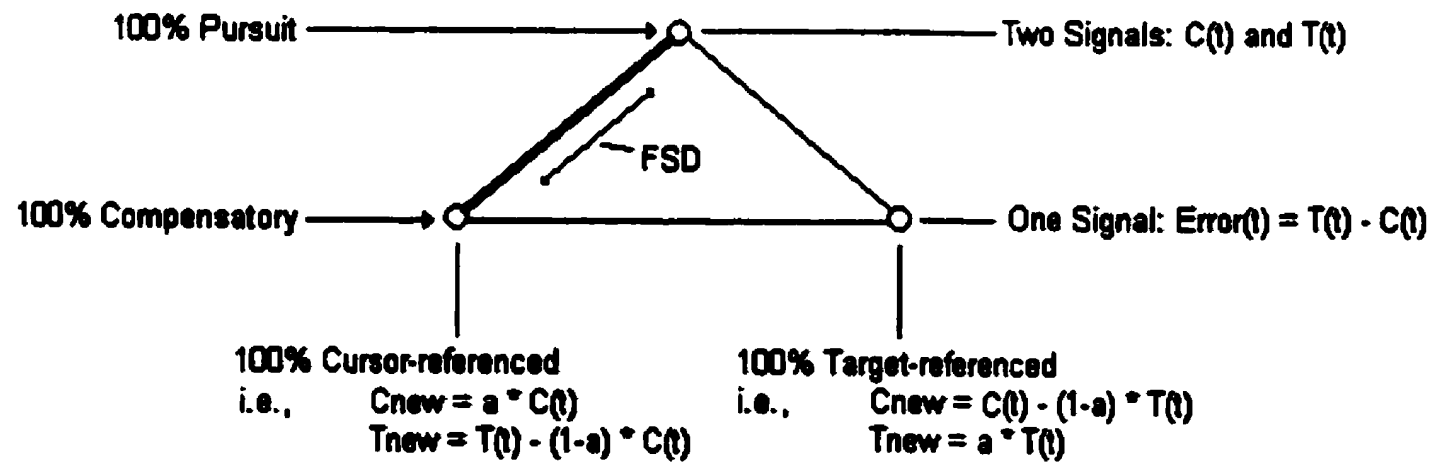

Figure 12 Specifying the mix of pursuit, cursor-referenced compensatory, and targetreferenced compensatory indications (adapted from Roscoe et al., 1981). The frequency separated display (FSD), in this report, traverses the left leg of the triangle. 
The following five conditions were tested in Senders' and Cruzen's experiment (as described in Roscoe et al., 1972):

(1) The cursor moved, and subjects tried to return it promptly and correctly to the stationary target. This condition provided a zero reference point, i.e. $0 \%$ pursuit, $100 \%$ compensatory combination.

(2) The ratio of the target movement to cursor movement was 1:3, i.e. $25 \%$ pursuit, $75 \%$ compensatory,

(3) Similarly, the ratio of the target movement to cursor movement was $1: 1$, i.e. $50 \%$ pursuit, $50 \%$ compensatory,

(4) The ratio of the target movement to cursor movement was 3:1, i.e. $75 \%$ pursuit, $25 \%$ compensatory,

(5) Only the target moved and the cursor remained stationary, unless moved by the subject, i.e. $100 \%$ pursuit, $0 \%$ compensatory.

For all subjects, the time-on-target score increased as the task shifted from compensatory to pursuit tracking. (The drawback of this study was that the investigators used time on. target as a measure of performance. This measure unfortunately does not increase linearly with an increase in performance.) There was no significant difference between the 75\%- and $100 \%$-pursuit conditions. The largest difference in performance occurred between the 0\%- (100\%-compensatory) and 25\%-pursuit conditions. These results indicate that a good deal might be gained by using a display that has the target spot (or conversely, the cursor marker as was done in this study) move slightly while maintaining the scaling advantages of compensatory presentations. It is this "scaling advantage" that 
has been manipulated to limit the degree of control-display incongruency permitted in the design of interfaces for the experiments in the present study.

In 1966 Briggs and Rockway, using modulus mean error rather than time on target, repeated the 1952 experiments of Senders \& Cruzen (cited in Roscoe et al., 1981). It was found that the pure compensatory display gave an average modulus mean error almost twice as large as that with the pursuit display. The display with the $25 \%$ pursuit component gave an average modulus mean error which lay about half way between the errors for $100 \%$ and $0 \%$ pursuit displays. Both sets of experimental results suggest that being able to see even an attenuated version of the track greatly improves tracking performance.

\subsection{Order of the Control System}

Tracking performance with a pursuit or compensatory display is also a function of the order of the control system used with it, as summarised in Table 2 . When the combination of a pursuit display and a rate control system is used in a tracking task, the operator's control movements must lead the target track by a phase angle of $90^{\circ}$ for optimal performance (Poulton, 1974). Whenever the track is irregular and/or contains high frequencies, however, it is not possible to predict the track in order to achieve such a large lead. Even if the track consists of only a single sine wave, the operator may be able to achieve the lead after much practice (and only for relatively low frequencies), but the movements he has to make will not be compatible with the observed movements of the track marker. That is, in 1/4 of a cycle the operator must move his control in the same 
direction as the track marker is moving. During the next $1 / 4$ cycle the operator must move his control in the direction opposite to the direction in which the track marker is moving. In the next $1 / 4$ cycle the directional relationship between control and display reverses again, and so on.

Predicting the track in this fashion with rate control is thus not trivial. However, using a position control system to perform the same pursuit tracking task can be even more cumbersome, even though no $\left(0^{\circ}\right)$ phase lead is required, especially if the amplitude of the track varies over a wide range of magnitudes, because the range of motion of the controller (not to mention the human operator) may be limited. That is, cursor motions may be large and thus require large controller motions, unless the gain is increased. However, increasing the gain makes tracking small target movements more difficult. The added ease and increased ability that is provided by using a rate control system thus offsets the error in predicting the target movements to produce the required phase lead. In addition, the following discussion will explain why a rate control system is more compatible with a compensatory display than a position control system.

Table 2 The phase relationships required between control order and display type (adapted from Poulton, 1974)

\begin{tabular}{|c|c|c|}
\hline 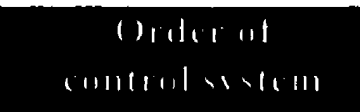 & 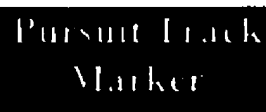 & 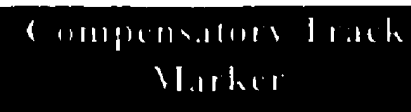 \\
\hline Position ( 0 "urder) & $0^{\circ}$ & $90^{\circ} \log$ \\
\hline Rate $\left(1^{1 x}\right.$ order $)$ & $90^{\circ}$ lead & $0^{\circ}$ \\
\hline
\end{tabular}

As indicated in Table 2, a position control system used in conjunction with a compensatory display requires the operator to reproduce the track with a phase lag of $90^{\circ}$ behind the actual movement of the error marker. A rate control system, however, 
automatically inserts a phase lag of $90^{\circ}$ into the operator's response, thereby directly providing the lag of $0^{\circ}$ is required for best tracking results. Thus a rate control system naturally matches a compensatory display in phase and better tracking performance should be expected with this combination. It can thus be concluded that rate control system reduces one of the disadvantages of a compensatory display. A detailed explanation of these controller-display task phase relationships can be found in Poulton's book (1974).

To reiterate, rate controls provide for easier tracking and a greater range of cursor motion than position control. However, a rate control system is difficult to use with a pursuit display because the operator essentially has to predict the track, with a phase lead of $90^{\circ}$ (see Table 2). As a display becomes more compensatory-like, on the other hand, using a rate control system becomes increasingly less detrimental than it is when using a $100 \%$ true-motion (i.e. pursuit) display, since there is no discrepancy in phase when using the rate control and the compensatory display. On the other hand, as discussed in previous sections, a compensatory display is also comparatively difficult to use, because the operator has to reproduce the track without seeing it.

In conclusion, when designing an interface for monitoring avatar/cursor actions, mixing display modes may be advantageous. That is, using a rate control system and mixing display attributes in the right proportion may result in optimal performance when the combined disadvantages of the pursuit and compensatory displays are minimised. Also, in applying mixed displays we should expect to find that changes in the phase 
relationships between the track and the response are required in order to track optimally. The above discussion describes how, when using a rate control system with a pursuit display, the operator's control input must lead the track by a phase lead of $90^{\circ}$ for optimum tracking performance, but no lead/ag is required when a compensatory display is used (see Table 2). Therefore more pursuit-like displays should produce input that lags the target track to a greater degree than that found in tracking performance with the more compensatory-like displays.

\subsection{Frequency Content}

Tracking inputs of low frequency reduce the disadvantages of using a compensatory display (Poulton, 1974). This is due to the fact that the period of these frequencies govern the amount of change that the forcing function introduces during the operator's latent reaction time. With lower frequency inputs, the operator is more accurately able to predict slow changes in the tracking signal more accurately relative to their reaction time. This advantage is relatively greater within the context of a compensatory display than with a pursuit display, because predicting the track ahead is ordinarily more difficult with a compensatory display. It follows therefore that reducing the frequency bandwidth in the tracking input should reduce both overall tracking error, as well as the difference in performance measures between compensatory and pursuit displays. Conversely, since tracking in any case becomes more difficult at higher frequencies, where movement rates are much faster, it is to the operator's advantage wherever possible to use a pursuit display for tracks containing high frequency components. 


\section{Frequency Separation Techniques}

Displaying information in an analogue format that both reflects the task environment and is also compatible with the user's mental representation is a necessary but not sufficient condition for ensuring maximum display compatibility. In the present project, the dual display concepts of direction compatibility and the principle of the moving part are considered, through the principle of frequency separation, as one possible method for increasing control-display compatibility. The frequency separation concept was originally conceived to address problems relating to outside-in displays (Fogel, 1959; Roscoe and Williges, 1975; Roscoe, 1981). As explained in the following section, when using a frequency separation algorithm, rapid changes in display properties are driven by the outside-in display principle, while relatively low frequency changes are driven according to the inside-out display principle (Stokes, Wickens, and Kite, 1990).

\subsection{The Principle of Frequency Separation}

In the particular domain of aviation, the frequency separation principle (FSP) has been proposed as a potential means of alleviating some kinds of frame of reference problems and some motion expectancy violations. In this section a review is given of some of the findings of past FSP studies in aviation, with an eye to how the principle may be applied to the domain of teleoperated ground vehicle control and navigation in virtual environments.

The technique of frequency separation was first implemented in aviation, notably through the work of Fogel (1959) and Roscoe (1981), who studied the application of frequency 
separated displays (FSDs) to the attitude-direction indicators of aircraft. In the following discussion it will be shown that pilot performance is facilitated by displays in which the symbol representing the pilot's own aircraft moves against a fixed reference system representing one or more dimensions of the outside world, that is, an outside-in display. The findings of several studies seem to indicate that these results are intimately involved with the dynamics of the display -- more specifically the high frequency component of the display indications. This is because it is critical that the elements of a display that respond immediately to the pilot's control inputs move in the expected direction. Conversely, it also appears that the direction of movement of the more slowly responding display elements is far less critical.

\subsubsection{Aviation Research involving Pursuit, Compensatory, and Frequency Separated Displays}

Several studies have been conducted measuring performance using various display modes in aviation. Time and time again the superiority of pursuit-type displays over compensatory displays has been demonstrated (Bauershmidt and Roscoe, 1960; Johnson and Roscoe, 1972; Poulton, 1974). The advantage of moving-aeroplane displays over moving horizon displays has also been illustrated more than once, as it obeys the principle of the moving part. Johnson and Roscoe (1972) disclose the history and present a very thorough discussion of the controversy surrounding the use of pursuit, compensatory, moving plane, and moving horizon displays. 
At the end of World War II much scientific focus was directed to the question of optimum movement relationships for flight displays. By studying the patterns of eye movements and fixations of pilots flying instrument guided approaches, it was discovered that the pilots spent most of their time looking primarily at three instruments: the attitude indicator, the heading indicator, and the cross-pointer indicator. It was noted, as well, that these three displays were the only ones in which the scale or some parts of the display representing the outside world moved (i.e. inside-out displays). In other displays where the scales were held stationary (i.e. outside-in), each position of the indicator had a unique meaning. This was not so with the moving scale inside-out displays. That is, it is the background that now embodies the unique position and meaning, and when the background is a scale which requires fine attention to be paid to the position of its numbers, direct perception of its position is more difficult. It is this direct perception of the object's unique position that was suspected to account for the differences in instrument dwell time. Thus an investigation was started. Among other things, it was found that the reading of outside-in displays, with fixed scales, was more accurate than the converse.

The moving horizon of the inside-out attitude direction indicator thus became a focus of debate. An aircraft's pitch and roll attitude is conventionally indicated by the position and movement of a small artificial horizon bar read against a fixed aircraft symbol. This presentation represents the geometric relationships one would see if one viewed the outside world through a small, circular porthole at the front of the aircraft. For example, whenever the aircraft rolls to the right, the artificial horizon rotates to the left, and vice 
versa. The position of the indicator thus maintains congruence with the real horizon, obeying the principle of pictorial realism (c.f. section 3.1.1). Many experiments compared this type of inside-out display with the converse, outside-in presentation, in which a miniature aircraft symbol moves against a fixed horizon line representing the outside world. The latter presentation is analogous to the geometric relationships one would see if one viewed the aircraft from behind and observed its movements against a fixed earth and sky. With such an outside-in display, whenever the pilot rolls his aircraft to the right, or clockwise, he would observe a rotation of his miniature moving aircraft symbol in the same direction.

Results of both simulator and flight experiments have generally favoured the outside-in moving-aeroplane presentation over the conventional moving horizon presentation, by a large margin (Bauershmidt and Roscoe, 1960; Johnson and Roscoe, 1972; Ince et al., 1975; Roscoe and Williges, 1975). In general, the frequency of steering errors was significantly reduced and the frequency of control reversals decreased when using the moving aeroplane outside-in display. Some of these results have been discounted, however, due to the fact that they were obtained in fixed-base (non-moving) simulators, rather than in a real aircraft. In two-degree-of-freedom moving-base simulators, on the other hand, pilots reported more difficulty with the outside-in moving aeroplane display than its inside-out moving horizon counterpart (Johnson and Roscoe, 1972). In conclusion, the issue of which display approach is better for aviation applications remains debatable. Nevertheless, in considering applicability of those past results to the current study, where we are applying this concept to teleoperation applications, for which the 
remote human operator is typically located away from the teleoperated vehicle or manipulator, we have much less reason to concern ourselves with possible kinaesthetic feedback interactions.

Returning to aviation, when flying straight and level or gentle flight manoeuvres the issue of motion compatibility may be of little concern. On the other hand, whenever the pilot's task requires sudden control movements, such as when recovering from unusual attitudes and or when flying complex tracking manoeuvres, control-display motion compatibility may become the primary factor governing successful execution. Evidence suggests that the critical consideration in this case is the relative speed of movement of the display elements. In particular, when high-frequency information is displayed, the moving element must respond in the expected direction. When low-frequency information is displayed, on the other hand, the initial direction of movement of the element apparently is not as crucial (Roscoe, 1968). These observations led to the development of the frequency separation concept.

Based on this concept Fogel (1959) demonstrated an attitude-direction display system called the "kinalog" display, in which the initial response of the attitude presentation followed the principle of the moving part. Fogel's design goal was to make the visually displayed attitude information more compatible with kinaesthetic and vestibular cues. Although there may be other reasons why an FSD algorithm may need to be implemented, the basic requirement is still the same: to increase control-display compatibility regardless of display modality (i.e. visual, haptic or kinaesthetic). Fogel's 
frequency separation was implemented by having display elements that respond immediately to aircraft accelerations (i.e. high frequency input) move in the same direction as the control inputs, while rate and position indicators (which respond at lower frequencies) move in the opposite direction. The aeroplane symbol in Fogel's display thus moves relative to the frame of the instrument, rolling with the aileron input and returning to the null position when the aileron control is neutralised. The horizon bar, on the other hand, slowly rotates in the opposite direction as the aeroplane rolls into a banked attitude, always indicating pitch and bank attitudes conventionally, according to inside-out principles.

Studies of these concepts suggest that control reversals are reduced and recoveries from unknown attitudes and steering performance are at least as good, and generally better, with the frequency-separated attitude display than with other displays (Roscoe and Williges, 1975; Ince et al., 1975; Beringer et al., 1975; and Roscoe, 1997). One study by Browne (1945) (cited by Johnson and Roscoe, 1972), for example, indicated that cadets in flight training required less time to learn and made fewer errors using a moving aeroplane display than the conventional moving horizon display. In a later study by Browne (1954), (cited by Johnson and Roscoe, 1972), however, this effect was eliminated by equalising the appearance and damping characteristics of the two displays. It is thus interesting to note that damping is an apparently important variable in the design of frequency separated displays. 
In spite of Browne's 1954 results, it can nevertheless be concluded that the frequency separation display concept should be considered in some form whenever control reversals are to be minimised and tracking performance maximised (Roscoe et al., 1981). Variations on Roscoe's FSD attitude display indicator by Payne in 1952 and by Hart and Wemp in 1979, for example, involved pilot control of the display by allowing the operator to rotate the orientation of a map physically or electronically (as cited in Roscoe et al., 1981). As further pursued in the present report, a similar exemplification of this idea is to allow operators to choose their own camera locations in a virtual environment and to tether that camera location continuously to the operators' avatar. Such an approach should thus permit the relative merits of each frame (of reference) to be exploited when most needed (Stokes, Wickens, and Kite, 1990).

\subsubsection{Outside the Cockpit}

Consider now a similar frequency separated aeroplane example: a map presentation with a vehicle heading "upward" on the display. If the vehicle turns to the right, involving a high frequency change, the plane symbol will also turn to the right. If the vehicle maintains the new course, involving a low-frequency behaviour, then the entire map slowly rotates counter-clockwise until the vehicle's new display heading is again upward, i.e. "track up". Such a system would preserve the principle of the moving part, essential for rapid transient inputs, while generally adhering to a compatible forward field of view of the outside world (for one who is inside the vehicle) by maintaining a heading-up rather than "north-up" orientation over the long term (recall Figure 5). 
This principle may also be extended to applications outside of the cockpit, such as teleoperation from a remote site. However, the reasons for implementing an FSD in this case differ slightly. Although preserving the principle of the moving part for rapid inputs remains a valuable asset, the principle of pictorial realism (c.f. section 3.1.1) may no longer be applicable. For teleoperation that removes the operator from a nominal viewpoint within the vehicle, the system no longer requires the operator to map the display to a "real" view of the surrounding environment. For cases in which an egocentric view may still be provided, for example by additional interface windows relaying video or a virtual view from the avatar's cockpit (or other nominal viewpoint), the principle of pictorial realism may still apply.

Another reason for implementing a FSD algorithm in a teleoperation interface is to maintain adequate control-display alignment. To do so requires a marriage of the "moving-window" method and the FSD technique. The dynamic tether model developed in this study is an ideal vehicle through which the principle of frequency separation and the method of the moving window may be combined. Liu, Chapter 51 in Salvendy, 1997) describe the moving-window concept in the following excerpt:

\begin{abstract}
"Although the principle of the moving part suggests the use of the moving-object method, the "moving-window" method is preferred when a software-user interface is used by the users to navigate in a large information space, only a small part of which is displayed and viewed at any time by the user. This method is not only necessary if the information space is too large to be displayed on the screen, but is also more consistent with user expectations in some task situations. An example of an application of the "moving-window" method can be found in the design of computer-based driving simulators: the direction of movement of the displayed objects is opposite to that of the control movement, accurately reflecting a user's experience in a driving environment."
\end{abstract}

The principles of the moving window and tethering are very similar in that the both are meant to preserve some of the characteristics of an ego-centred viewpoint and both 
involve scrolling of the background through the given viewpoint to preserve global awareness. The general idea of combining these concepts with the tethering concept, in this study, is to investigate if doing so will reduce the mental information processing involved in a task execution and, thus, improve performance.

\subsubsection{Information-Processing Models and Control Theories}

The human operator is one of many components in a typical tracking control loop (see Figure 17). One of the ways in which the operator can be represented is by an information processing model (Knight, chapter 2.7 in Salvendy, 1987; Wickens, 1992). As illustrated in Figure 13, such a model should, at the least, encompass perception, decision making and response control, all of which are important in performing tracking tasks. In addition, one of the other elements of such a control loop that should influence tracking performance is the manner in which the target system's state is displayed to the operator. All of these elements are taken into consideration in this study, as the application of frequency separation in display design is used to create a display that accommodates good tracking performance.

Control and tracking performance depend upon the information-processing capacities of the three major stages of the model, and thus is limited by them. Furthermore, any stage in the information processing model can transmit information at its maximum rate only if the information is coded in the proper format (Knight, chapter 2.7 in Salvendy, 1987). If additional transformations must be performed to produce an appropriate response, then the rate at which information is handled by the overall system will be below maximum. 
Examples of such transformations, which are of interest in the present study, include mental rotations and derivative computations. Other transformations, such as integration for selecting appropriate control responses, are not of interest ${ }^{1 !}$.

The need to perform complex data transformations imposes a particular demand upon the decision-making stage of information processing. In general, reducing the degree of processing required to map input information into a desired response improves information transmission rate and thus improves control performance. The application of tethering and the concept of frequency separation within the interface design of this study represent an attempt to minimise any additional processing required for mental rotation (as encountered in a non-tethered display) and scene integration (as required by an egocentric display). Dynamic tethering (which will be described later) attempts to maintain the advantages of traditional tethering while reducing the need for the computation of derivatives of presented information to determine the effects of previous responses to operator input.

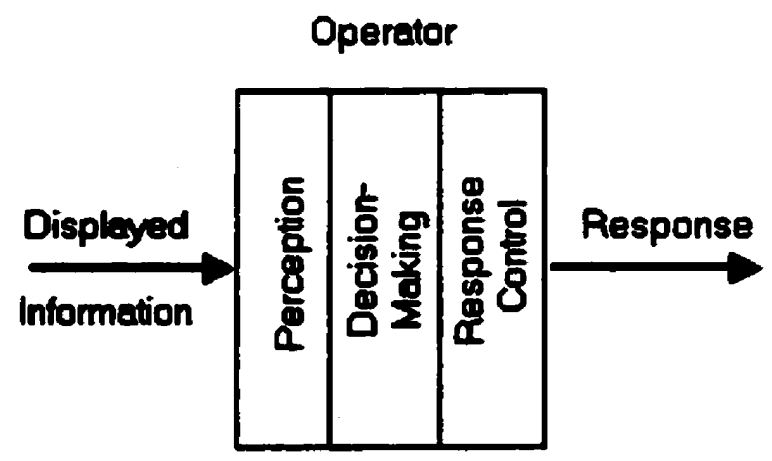

Figure 13 A simplified information-processing model of the human operator (adapted from Knight, chapter 2.7 in Salvendy, 1987) 
Another information processing limitation that can have harmful effects on performance is the time delay that separates successive outputs from the decision-making stage. This delay is often modelled as a fixed 300 ms psychological refractory period (PRP); that is, if new information is presented within $300 \mathrm{~ms}$ of a previous decision, decision making is delayed until the PRP has elapsed (Knight, chapter 2.7 in Salvendy, 1987). This refractory delay does not decline with practice. The PRP can, however, be reduced when stimulus-response compatibility (S-R) compatibility is high. S-R compatibility refers to the "naturalness" of the relationship between a stimulus and the response with which it is associated. High S-R compatibility may reduce the PRP delay to about $200 \mathrm{~ms}$. At a period of $\mathbf{2 0 0} \mathrm{ms}$ an operator is thus capable of making 3-4 decisions per second. Given that a single sinusoidal track will undergo two changes in direction per cycle, one may therefore predict that, assuming serial processing and excluding any additional processing requirements, an operator is theoretically capable of successfully tracking signals with a maximum frequency of only $1-2 \mathrm{~Hz}$. Again, it is stressed that providing information selection mechanisms and efficient information transformation formats may reduce the amount of mental loading, increase information flow rate, and thereby increase tracking performance. Empirical tracking studies have in fact revealed that operators can effectively track continuously varying input signals not exceeding a bandwidth of approximately $1 \mathrm{~Hz}$ (Knight, chapter 2.7 in Salvendy, 1987). (The input tracking frequencies presented to subjects in the present study were kept below this value for this reason.) This $1 \mathrm{~Hz}$ upper limit on operator performance is thus the highest possible input frequency that frequency separated displays that respond to operator control input may 
see (i.e., the break point in the frequency separation algorithm should never be set higher than $1 \mathrm{~Hz}$ ).

In addition to the maximum frequency that the operator is able to process, the minimum frequency that an operator is able to perceive is of equal importance. As a general rule, when tracking frequencies are so low that performance does not differ significantly between pursuit and compensatory displays, compensatory display should be used because of their scaling advantages, which also allow control-display alignment to be preserved. However, when the frequency content of the input increases to the point where pursuit display characteristics (i.e., easily discriminable cursor and target movements) begin to counter associated decreases in task performance, then pursuit displays should be used. In situations where the requirement for both mental transformation and discriminable motion perception is high, some combination of the two display formats should be considered.

A second order control system has the desired response characteristics of a combined pursuit-compensatory type display. That is, a second order transfer function (those with complex conjugate poles being of interest in this report) of a system has a frequency response that attenuates high frequencies while it reacts to low frequency inputs (Dorf and Bishop, 1995). Figure 14 shows a set of Bode plots of the frequency responses of a set of second order systems. The plots indicate that for damping ratios, $\zeta$, close to 1.0, the gain of the transfer function is approximately $0 \mathrm{~dB}$ (or 1.0) for input frequencies below both the natural and resonant frequencies ( $\omega_{n}$ and $\left.\omega_{t}\right)$ respectively. As the input 
frequency approaches and then surpasses the natural frequency (indicated by a frequency ratio of $\omega / \omega_{n}=1.0$ along the abscissa), the system gain drops off and thus the system response to any input diminishes. Such systems thus perform as an effective low pass filter. The bottom plot in the figure indicates the phase lag associated with the given frequency ratio.

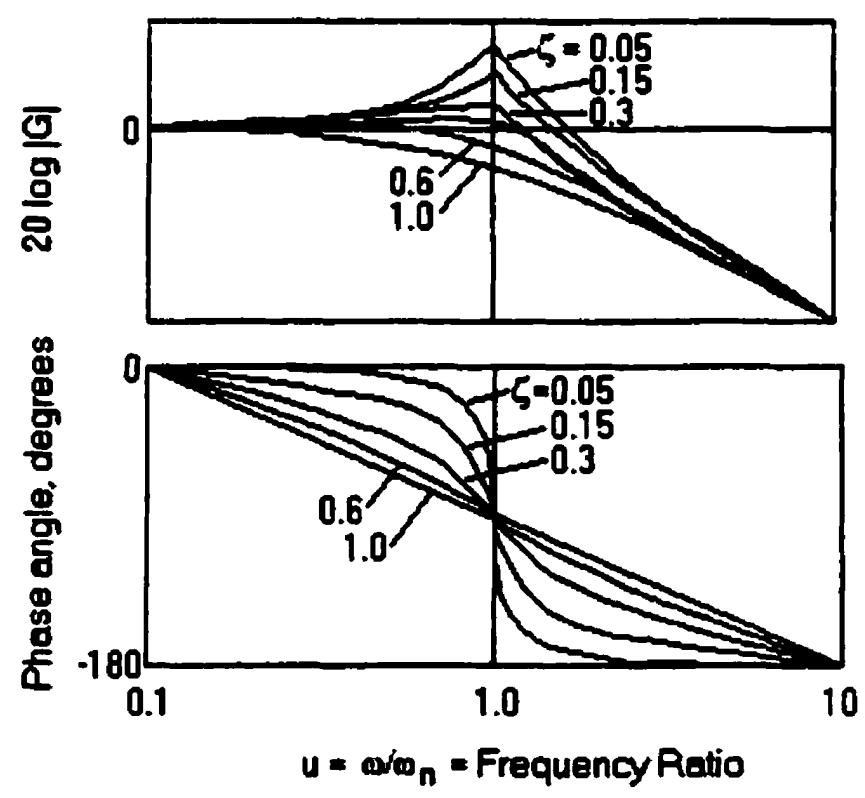

Figure 14 Bode diagrams of typical frequency responses for a set of second-order system transfer functions, $G(j \omega)$, as a function of damping coefficient, $\zeta$,

Combining the concepts of pursuit and compensatory displays with the frequency response of a second order system (such as a mass-spring-damper system) can lead to some interesting applications. The parallel between the pursuit-compensatory display continuum (c.f. section 4.1) and the high-to-low frequency response characteristics of the second order system are as follows:

1. At one extreme, a pursuit display allows the operator to perceive both cursor and target movements in a separable manner, thus tracking of high frequency components 
is generally performed better with a pursuit display than with a compensatory display.

Concurrently, second order systems respond relatively little to high frequency inputs.

In summary, therefore, at high frequencies both second order system response and compensatory display advantages are minimal.

2. At the other extreme, a compensatory display confounds cursor and target motions, but allows the operator directly to perceive the error between the two, which is advantageous at low frequencies because of the display's scaling properties. Concurrently, second order systems respond maximally to low frequency inputs. In summary, therefore, at low frequencies both second order system response and compensatory display advantages are maximal.

Table 3 Summary of complementary display characteristics that are encompassed in the model of the dynamic tether.

\begin{tabular}{|c|c|c|}
\hline $2^{\text {110 }}$ order system & - maximal response & - minimal response \\
\hline $\begin{array}{l}\text { Pursuit (type I) } \\
\text { Display }\end{array}$ & $\begin{array}{l}\text { no prevention of control-display } \\
\text { incongruencies } \\
\text { no significant atuantage over } \\
\text { compensatory displays }\end{array}$ & $\begin{array}{l}\text { true nature of cursor and target } \\
\text { marker motions is more } \\
\text { perceptible } \\
\text { enhances high frequency } \\
\text { tracking. }\end{array}$ \\
\hline $\begin{array}{l}\text { Compensatory } \\
\text { Display }\end{array}$ & $\begin{array}{l}\text { error signal (relative motion) alone is } \\
\text { adequate for efiective tracking, provided } \\
\text { cursor motion can be induced by } \\
\text { background motion, } \\
\text { but compensatory-like display also has } \\
\text { scaling characteristics that may be used } \\
\text { to maintain some degree of control- } \\
\text { display congruency }\end{array}$ & $\begin{array}{l}\text { not enough marker motion } \\
\text { information is provided for } \\
\text { adequate tracking performance. }\end{array}$ \\
\hline 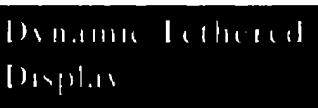 & 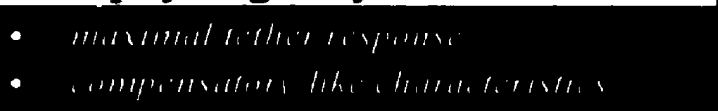 & 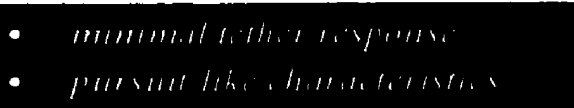 \\
\hline
\end{tabular}


This parallelism is summarised in Figure 14, which is intended to convey the message that creating a display in which a second order system is used to drive the compensatory characteristics of the display (i.e. at low frequencies) should satisfy the display needs of the control tasks described in this paper. One method of manifesting such properties in the relationship between the display and its moving markers (i.e., a dynamic tether) is described in section 5.2 .

\subsubsection{Motion perception and Applying the Frequency Separation Technique}

Given that a second-order transfer function may be used as a FSD algorithm, it must be determined how to optimise the frequency separation parameters relative to the task, that is, to determine where the break frequency of the FSD algorithm should be located with respect to the frequency content of the task domain. One important factor to take into account is the relationship between the frequency content of the display and the capabilities of people to perceive certain frequencies and associated velocities of motion. Of particular interest in this study is the ability to perceive subject-relative and objectrelative visual motion (Section 5.201 in Boff \& Lincoln, 1988). It is thus hypothesised that the optimal break, or natural, frequency is constrained by human perceptual ability, i.e., the minimal perceptible angular velocity for real motion and velocity threshold for motion perception - which may define the lower bound, and the $1 \mathrm{~Hz}$ upper limit on operator performance outlined in section 5.1.3. Exactly where that "break-frequency" is located within these bounds remains a function of the frequency content of the task space.

Subject-relative motion gives the observer information about the absolute motion of an object relative to the observer and is drawn both from retinal-image motion and from 
information about movement of the observer's own head and eyes. Object-relative motion information, on the other hand, is available from relative changes in the position of retinal images of objects. By itself, object-relative motion reliably indicates that one or more objects are moving relative to each other, but it does not specify which one; therefore such information is ambiguous in the absence of subject-relative information. The information extracted from object-relative motion is therefore analogous to the information provided by a compensatory display.

The purpose of implementing an FSD algorithm in this study is to separate critical high frequency motion information from less critical low frequency motion information (c.f. sections 5.1.1 and 5.1.3) in an attempt to avoid control-display incongruencies. The thresholds of motion perception may be used as a first estimate of the optimal location of the break frequency. Because thresholds for clockwise and counter-clockwise movement detection are not significantly different (Section 5.206 in Boff \& Lincoln, 1988), it is presumed that a symmetrical FSD implementation is required.

In general, the threshold for visual movement perception is influenced by many factors, such as target velocity and distance, reference frames, visual fixation and/or visual pursuit. Furthermore, observers are more sensitive to object-relative than subject relative motion(Section 5.201 in Boff \& Lincoln, 1988). The minimum angular velocity at which motion can be detected is dependent upon the presence of background information. When a textured background is present, motion can be detected at 1 to 2 minutes of arc of visual angle per second, an order of magnitude lower than when a target moves against a 
featureless background, i.e., 10 to 20 minutes of arc per second (Section 5.201 in Boff \& Lincoln, 1988). In any case, whenever cues conflict, object-relative motion dominates perception.

The second threshold of motion detection that is of interest is that of induced motion. Induced motion is the perceived motion of objects that are not actually moving, whenever other objects are moving instead. This measure is important in determining the range of background velocities that may induce cursor motion when compensatory type displays are used. While real motion can be detected at velocities greater than 25 to 50 arc seconds of visual angle per second, induced motion perception occurs at a higher threshold, and only between a limited range of velocities. The lower threshold is between 60 and 120 arc seconds per second, and the upper threshold is between 10 and 50 arc minutes per second.

Since motions with frequencies lower than 50 arc sec $/ \mathrm{sec}$ are not perceptible, the optimal break frequency must be larger than this value. In this study, break frequency values of 2, 10 and 50 minutes of arc of visual angle per second were investigated. It was assumed that cursor motions below these break frequencies would be confounded with target motions, while those motions at higher frequencies will be displayed as true cursor and target movements.

\footnotetext{
II As discussed later, a rate control device has been used throughout this study. Since the order of control (i.e. $1^{*}$ order) has also been kept constant, any need for mental integration, due to control order, is kept consistent over all tasks.
} 


\subsection{The Dynamic Tether}

The experiments presented in this report involve the application of a FSD in the form of a dynamic tether. The results of past experiments in the literature indicate that this technique should:

(1) benefit both global navigation and local control by reducing the degree and number of mental transformations required in performing a task;

(2) take advantage of the close cognitive connection posited between the directions "upward" and "straight ahead";

(3) automate the compensation required when changes of frame of reference are made;

(4) mediate many of the practical problems associated with map interpretation.

\subsubsection{Modelling the Dynamic Tether}

Modelling the dynamic tether as a spring-mass-damper system mirrors the concept of impedance control discussed by Sheridan (1994). Impedance control is used to ensure smooth transitions in robotic control by varying the velocity of robot motion between any two points. Figure 15 illustrates a hypothetical end-point compliance frame for example. The transfer function for impedance (Z) defines the relationship between an applied force (F) and resulting velocity (V):

$$
\begin{aligned}
Z(s) & =F / V=M s+C+K / s \\
& =F /(s Y(s))=M s+C+K / s \\
F(s) & =M s^{2} Y(s)+C s Y(s)+K Y(s)
\end{aligned}
$$


where $M$ is mass, $C$ is viscous damping, $K$ is stiffness, and $s$ is the Laplace argument.

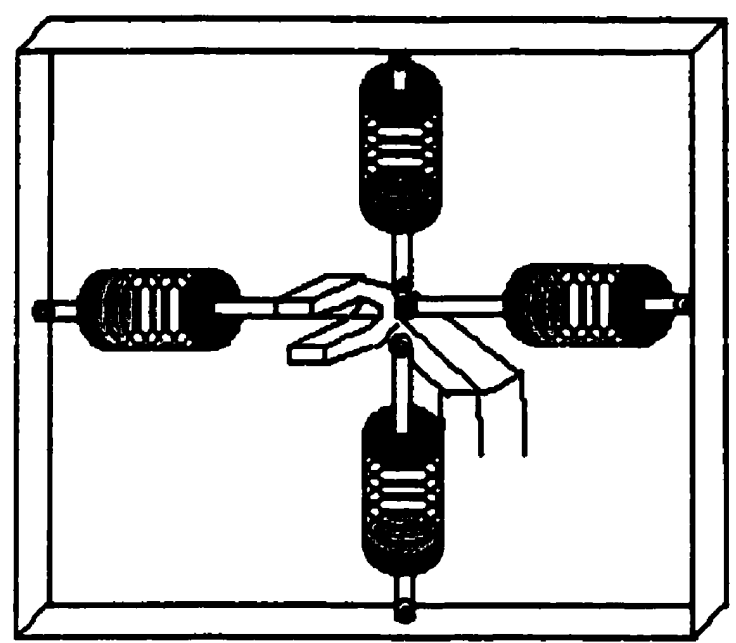

Figure 15 Illustration of Sheridan's compliance frame.

(Adopted from Sheridan, 1994).

The impedance in each axis of control may be manipulated by varying the compliance (stiffness) of each component connected to the endpoint of the teleoperator arm in Figure 15. In this example the compliance null point, stiffness $(\mathrm{K})$, and viscosity (C) parameters can be adjusted independently. The adjustment of mass $(M)$ in such a system is usually neglected (Sheridan, 1994). Equation 5-1 through 5-10 shows the similarity between impedance control and the transfer function of a general spring-mass-damper (i.e. second order) system. As in equations 5-1 to 5-3, the force equations can be written as differential equations:

$f(t)=M\left(d^{2} y(t) / d t^{2}\right)+C(d y(t) / d t)+K y(t)$

(Equation 5-4)

Writing this in the Laplace domain and assuming zero initial conditions

$F(s)=M\left(s^{2} Y(s)-s y(0)-d y(0) / d t\right)+C(s Y(s)-y(0))+K Y(s)$

(Equation 5-5) 
$F(s)=M s^{2} Y(s)+C s Y(s)+K Y(s)$

(Equation 5-6)

The force-velocity transfer function then becomes:

$$
\begin{aligned}
\mathrm{Y}(\mathrm{s}) / \mathrm{F}(\mathrm{s}) & =1 /\left(\mathrm{Ms}^{2}+\mathrm{Cs}+\mathrm{K}\right) \\
& =(1 / \mathrm{M}) /\left(\mathrm{s}^{2}+(\mathrm{C} / \mathrm{M}) \mathrm{s}+\mathrm{K} / \mathrm{M}\right) \\
& =(1 / \mathrm{M}) /\left(\mathrm{s}^{2}+2 \zeta \omega_{n} s+\omega_{n}{ }^{2}\right)
\end{aligned}
$$

where $\omega_{\mathrm{n}}=(\mathrm{K} / \mathrm{M})^{1 / 2}, \zeta=\mathrm{C} /\left(2(\mathrm{KM})^{1 / 2}\right)$, and $\left(\mathrm{s}^{2}+2 \zeta \omega_{n} s+\omega_{n}^{2}\right)=0$ is the second order characteristic equation which governs the system dynamics.

If $F(s)$ can be expressed as $M\left(s^{2} X_{\text {in }}(s)\right)$ then we obtain the following relationship between input displacement, $X_{\mathrm{in}}(\mathbf{s})$, and output displacement, $Y(s)-$ that is, the transfer function:

$$
\frac{Y(s)}{X_{m}(s)}=\frac{s^{2}}{s^{2}+25 \omega_{n} s+\omega_{n}^{2}}
$$

Equation 5-10 is the same equation that governs the dynamics of the parallel springdamper arrangement in Figure 16.

Just as control over the end-point of a manipulator, as shown in Figure 15, can be accomplished by moving the compliance frame, thereby applying a force to it, a similar compliance frame may be affixed to the end-point and connected to a viewpoint, as illustrated in Figure 16. Such a frame would then determine the dynamics of camera viewpoint changes resulting from movements of the end-effector. This is the idea behind 
the frequency separation concept as it is modelled and implemented within this study. Just as with the compliance frame in Figure 15, the compliance, viscosity, and mass parameters of the "compliant tether" may be adjusted to obtain desired reactive motions, which are subject to the constraints of stability and dynamic limitations of the system. Given this flexibility, these parameters may be changed in different directions, or even changed with time if necessary. (On-the-fly adjustment of this "dynamic" or "compliant" tether is beyond the scope of this paper; however, it could become the topic of future research.)

Tethering the virtual camera to the virtual avatar via a mass-spring-damper system is similar to passing the motion of the "camera" through two lowpass filters connected in series. This produces a sigmoid type lag in the camera response to system output (see the phase plot of Figure 14). In fact, the characteristics of the dynamic tether have a fascinating resemblance to the characteristics of the human operator as predicted by the crossover model. The crossover model is a linear human operator transfer function model which describes control performance within the frequency region where the open loop gain is approximately $0 \mathrm{~dB}$ (i.e., a factor of 1.0 ) (Wickens, 1986). The crossover model postulates that the human contribution to the control loop is such that the combined humantsystem open loop transfer function adopts a high gain at low frequencies, to ensure good control accuracy, and adjusts the gain at the crossover frequency to preserve the stability margin. Similarly, the dynamic tether creates a link between a controlled object and the viewpoint. This link has relatively high gain characteristics at low frequencies, to maintain control-display congruence, and low gain 
characteristics at high frequencies, to allow the operator to perceive velocity of target motion, so as not to adversely affect tracking performance.

The ideal characteristics of the dynamic tether are as follows:

(1) The system should be approximately critically or over-damped $(\zeta>0.7)$, as perceptible viewpoint oscillations are undesirable and would degrade performance.

(2) The response should be slow to start to support visual momentum (i.e., a smooth transition) between viewpoints and then accelerate to steady state.

(3) As little overshoot as possible should be achieved, without excess slowing of the rise time to steady state.

(4) The settling time (the time it takes the viewpoint to align with the its steady state position) should be minimal.

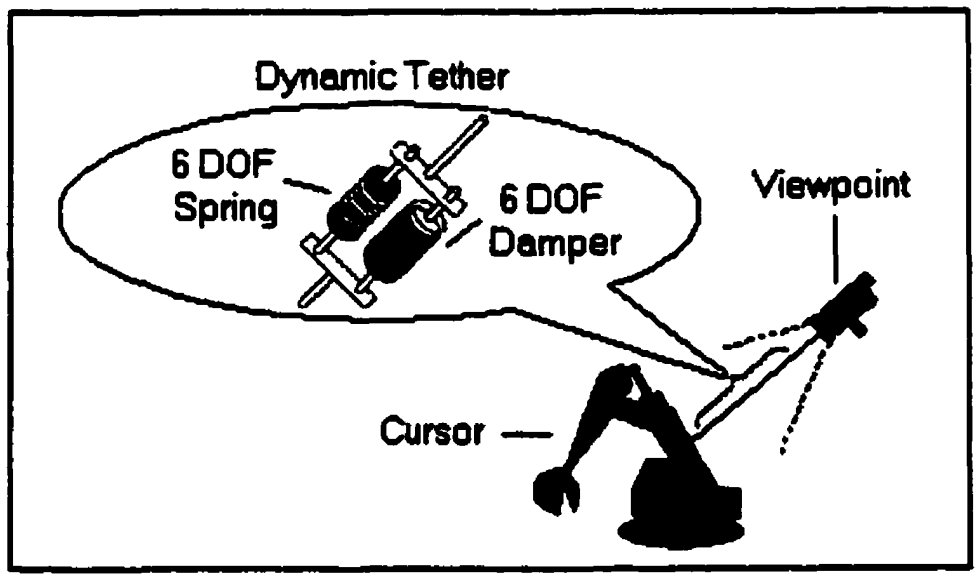

Figure 16 The mechanical analogue of the dynamic tether (a second-order system)

Dynamic tethering combines the tethering concept (which also implicitly describes the moving-window method - c.f. section 5.1.2) with the frequency separation concept. That 
is, frequency separation in this study describes variable mixing of percentage pursuit versus compensatory tracking, as illustrated along the left side of the triangle in Figure 12 , with respect to input frequency.

Having established a connection between dynamic tethering and pursuit/compensatory displays, we may now proceed to apply the wealth of information available in the literature on the two concepts to predict the theoretical advantages. For example, it has been shown that a conventional pursuit display has three major advantages over a conventional compensatory display (Poulton, 1974):

(1) Pursuit displays facilitate prediction of future movements of the tracking input more accurately, thereby reducing time lag.

(2) Pursuit displays facilitate easier learning of the control system and the monitoring of its output.

(3) Pursuit displays facilitate the avoidance and detection of mistakes, since status information is presented in a naturalistic form.

Performance with a pursuit display benefits from its compatibility with human spatial perception. In particular, the eyes can readily perceive the true motion of two markers against a structured background, as well as their motion relative to each other when they are in close proximity; that is, a pursuit display makes use of both perceptual capacities simultaneously. A compensatory display, on the other hand, makes use only of the capacity of the eyes to perceive relative motion (i.e., changes in the directly displayed error signal). The greatest benefits from pursuit displays are therefore obtained when the 
two markers are fairly close to one another and move against a well-structured background. Background texture, or calibration markers against which the markers move, or the edges of the display may all provide such "structure".

The visibility and proximity of the background structure traditionally determines the type of display being used. Poulton's (1974) example of a newsman reporting with a television camera illustrates this idea. If the news reporter is broadcasting a race, then the lead racer is tracked with a moving camera sight. This is a pursuit (true motion) task, because the newsman perceives both the racer and the sight crosshairs moving along the track, but actually, within the camera viewpoint, it is the racer and the track that move while the crosshairs remain stationary with respect to the walls of the sight. We define this case operationally as a pursuit type II display ${ }^{12}$. Suppose now that he is instead tracking an aeroplane flying in a clear sky with the same sight's crosshairs. In this case, there is no structured background for either object to move across so, when tracked perfectly, the aircraft appears motionless on the crosshair at the centre of the sight. This case thus corresponds to a traditional pure compensatory display. However, if the aircraft flies low enough such that the ground now becomes visible, the display changes again to true motion or pursuit type II display.

Recall that a traditional pursuit, or operationally pursuit type $I$, display involves true motion of both the cursor and target markers against a background whose motion, if any, is independent of that of the markers. Comparing the pursuit type II displays in the above newsman example to a pursuit type I display in which the background does not move (i.e. 
true motion display) is of particular interest in this study. When the background is stationary - relative to the display - and the cursor and target are seen to move against this background the motion is most intuitive and maximal efficiency is achieved in perceiving both relative and true motion. However, when the background is not stationary (with respect to the frame of the display) and either the cursor or target are made to be stationary relative to the display (i.e., a zero marker) the author suggests that this display has become more like a compensatory (or pursuit type II) display. In this case, however, the information required for extracting relative and true motion is still present, but is not always intuitive, due to the potential control display motion incompatibility that may sometimes arise between the direction of movement of the cursor controls and the direction of movement perceived on the screen. If the effect of apparent motion is strong, then there might not be much difference in performance between either of the two types of true-motion displays. However, if apparent motion is not achieved successfully, the background information may be ignored or, at worst, interpreted incorrectly. If this is the case, the display corresponds to a compensatory display and the background information might as well not be presented, because the operator perceives only the error (relative motion) but not the true motion information.

Table 4 summarises the characteristics of the three types of tracking displays discussed in this report. In tethering the viewpoint (camera) to the cursor we create motion of the background. This motion may or may not cause induced motion of the stationary cursor or target, based on several factors, including perception of angular velocity. If it does, then the advantages of the pursuit display (type II) are preserved. If it does not, then the 
display corresponds effectively to a compensatory display. In the current study a structured background is provided. Future research should be conducted to determine the extent of the effect of adding background information to a purely compensatory display.

\section{Table 4 The relationship between display type and display component motion:}

A traditional pursuit (type I) display permits the operator to perceive the true motion of the display markers on a stationary background (all with respect to a fixed display frame). Thus the cursor moves with control input velocity $\mathbf{C}$ and the target moves with track input velocity $\mathrm{T}$. When a pursuit type II display is used cursor motion is instead induced by background movement relative to the display frame. Thus the cursor does not move with respect to the display frame, but the background moves with velocity $-\mathrm{C}$ and the target moves with velocity T-C. The compensatory display described in this report refers to that in the fourth row. The difference between the pursuit type II display and the traditional $100 \%$ cursor referenced compensatory display is that the latter does not have a background texture.

\begin{tabular}{|c|c|c|c|c|}
\hline H,yll, I & 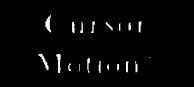 & $\begin{array}{l}1.11211 \\
1+1+1,1\end{array}$ & 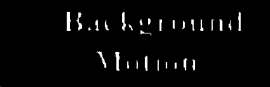 & 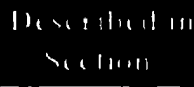 \\
\hline $\begin{array}{c}\text { Pursuit Type I } \\
\text { (100\% True Motion) }\end{array}$ & Yes $(+C)$ & Yes $(+T)$ & No (0) & 5.2 .1 \\
\hline $\begin{array}{c}\text { Pursuit Type II } \\
\text { (Compensatory-like Display with } \\
\text { Background Induced } \\
\text { Cursor Motion) }\end{array}$ & No $(0)$ & Yes (+T-C) & Yes $(-C)$ & 5.2 .1 \\
\hline $\begin{array}{c}\text { Compensatory } \\
\text { (Relative Motion, } \\
100 \% \text { Target Referenced) }\end{array}$ & Yes $(+C-T)$ & No $(0)$ & $\begin{array}{l}\text { No background } \\
\text { texture present } \\
\text { (theoretically, }-T \text { ) }\end{array}$ & - \\
\hline $\begin{array}{c}\text { Compensatory } \\
\text { (Relative Motion, } \\
100 \% \text { Cursor Referenced) }\end{array}$ & No (0) & Yes (+T-C) & $\begin{array}{l}\text { No background } \\
\text { texture present } \\
\text { (theoretically, }-C \text { ) }\end{array}$ & 4.2 \\
\hline
\end{tabular}

The presence of motion relative to the display frame with velocity in brackets $(C=$ cursor velocity, $T=$ target velocity, $0=$ stationary)

Figure 17 shows how dynamic tethering may be modelled within the context of an operator-plant control loop. The variable $A$ is a function of break frequency $\left(\omega_{n}\right)$, and damping ratio ( $\zeta$ ), and varies between 0 and 1 . When $A$ has a value of 1 , this corresponds to the case of a textured, non-moving background. The backdrop position thus does not provide any information $(1-A=0)$ and the display mode is $100 \%$ pursuit (type $I)$. In other words, the operator perceives the tracking input and the cursor position information 
separately. Conversely, when $A$ has a value of 0 , the cursor position is not perceived directly and the display mode is thus $100 \%$ pursuit type II (compensatory-like with a textured background). Figure 17 also defines the relationship between the break frequency $\left(\omega_{n}\right)$, damping ratio $(\zeta)$, and the target, backdrop, and cursor positions.

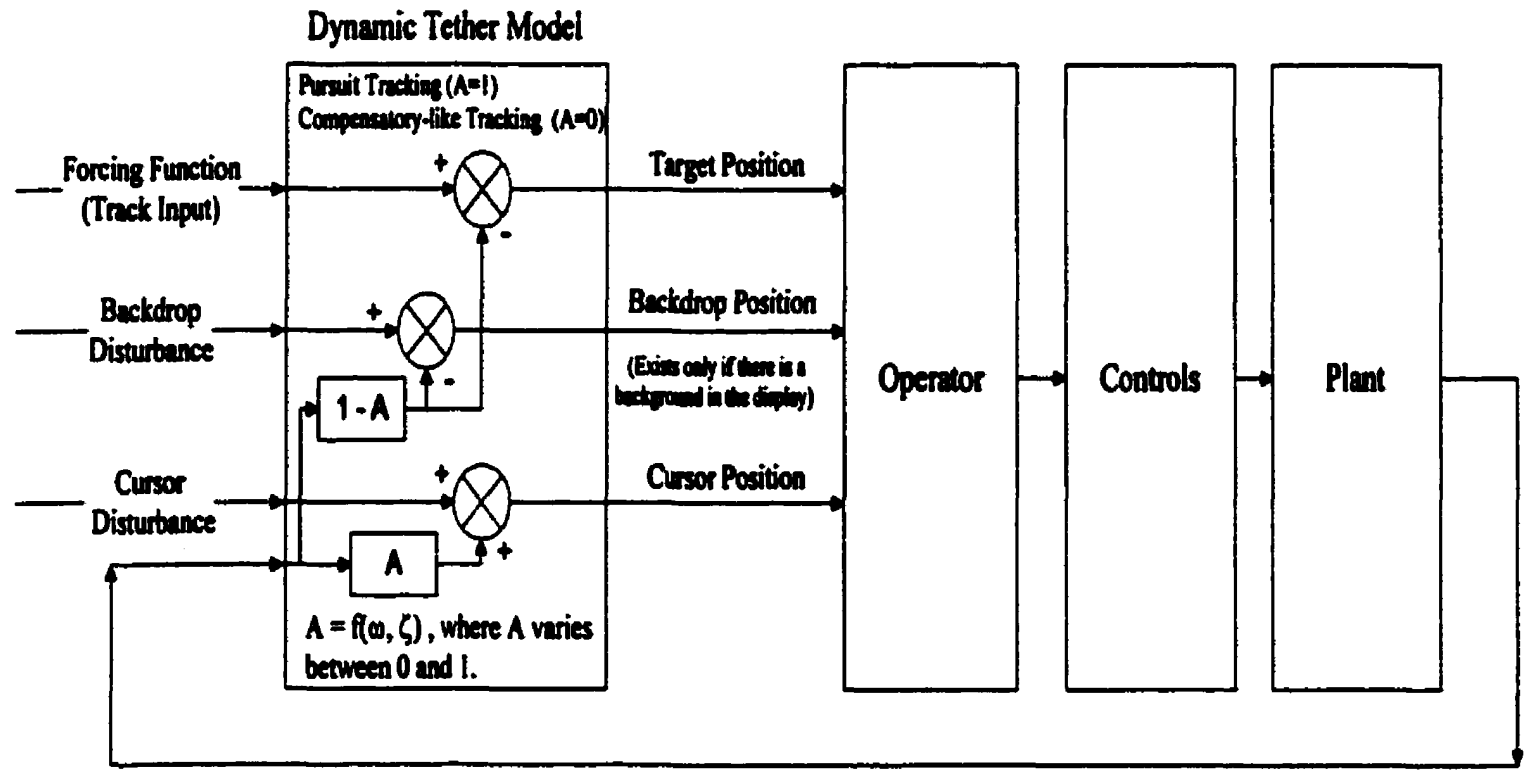

Plant Position Output

Figure 17 Control Loop for Dynamically Tethered Frequency Separated Tracking

The relationship between centricity and scene motions can also be illustrated in terms of the dynamic tether. As was illustrated in Figure 7, centricity varies with the displacement of one's viewpoint from some nominal viewpoint associated with the object being controlled. This characteristic is also illustrated in Figure 6. However, in addition, Figure 6 also illustrates the relationship between centricity and scene movement. Recall that, when a highly egocentric display is provided, the moving part of the display is the outside world. Conversely, with a highly exocentric display, the moving part is the object being controlled. Tethering (with a non-zero length tether) makes it possible to 
view the controlled object exocentrically while maintaining an egocentric motion relationship. The perceived movement of the viewpoint with movement of "ownship" is referred to as egomotion. Therefore, two orthogonal aspects of centricity may be associated with the dynamic tether:

(1) viewpoint displacement from the nominal viewpoint (i.e., the length of the tether), and

(2) egomotion defined by the coupling between the viewpoint and the nominal viewpoint (i.e., the stiffness or rigidity of the tether).

These concepts are summarised in the two dimensional space described in Figure 18.

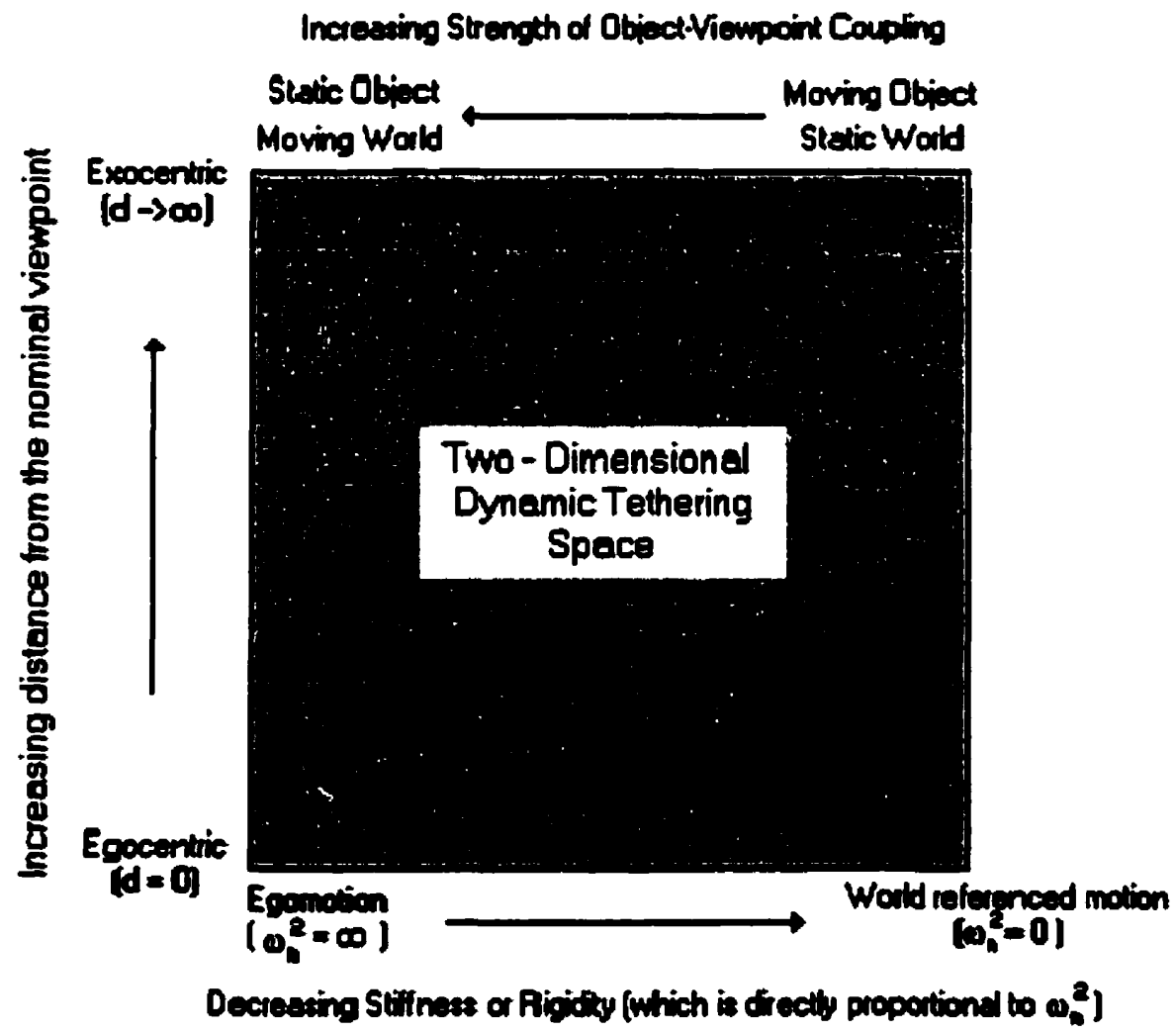

Figure 18 Two-dimensional dynamic tethering space, encapsulating both tether displacement (d) and coupled motion aspects of centricity 


\subsubsection{Dynamic Tethering and the Praximity Compatibiltity Principle .}

The traditional Proximity Compatibility Principle, or PCP, deals specifically with the issue of display organisation (Wickens and Andre, 1990; Wickens, 1992). "In general, the principle holds that ... indicators or displayed data values that are conceptually related, or that need to be used in combination, should belong to the same perceptual group. In short, related information should be perceptually proximate." (Bennett, Magy, and Flach, in chapter 20 of Salvendy, 1997). Although in the past PCP has dealt with defining proximity in terms of space, connectivity, code homogeneity, and feature similarity, it is proposed here that the concept may be extended to include temporal aspects as well (as does Burns, 1998).

In invoking the concept of "temporal display proximity", we are referring to similarities between the dynamic behaviour of different elements of a display. In particular, just as the principle of proximity compatibility emphasises the relationship between task demands and the graphical form of the display, the dynamic tether unifies (as per the PCP) the compatible format characteristics of rigidly tethered displays (i.e. pursuit type II or compensatory-type displays) and the separable information characteristics of nontethered displays (i.e. a pursuit-type displays) in time. In this sense the high temporal display proximity of the dynamic tether should prove helpful in tracking tasks with high mental proximity, since in a high mental proximity task, information from both sources must be integrated to perform the task effectively. "To the extent that the information must be treated separately (low mental proximity, as when one must focus on one 
variable and ignore values of the other), the benefits of the high-proximity ... display will be reduced, if not sometimes reversed." (Wickens, 1992).

The advantage of the dynamic tether is that its dynamics may be tuned, such that either the compensatory display mode or the pursuit display mode or some ratio of each is presented, as required for optimal human-machine task performance. Thus, a dynamic tether of intermediate rigidity will incorporate some of the features of similarity for nonintegrative processing, which is an intermediate level of processing proximity (Bennett, Magy, and Flach, in chapter 20 of Salvendy, 1997). Processing proximity, or mental proximity, refers to the continuum of attentional demands, that is, the extent to which information from various sources in a display must be considered together (or not) to accomplish a particular task. The three such features of similarity inherent in the dynamic tether are:

(1) functional similarity or semantic relatedness,

(2) processing similarity, i.e. the similarity of computational procedures, and

(3) temporal similarity/proximity.

\subsubsection{Motion Detection}

Implementation of the dynamic tether has been expressed here as a mixture of compensatory and pursuit-type displays. It has also been shown that the pursuit-type display provides the operator with more (separable) information about the dynamics of the task space with which he is interacting than the compensatory-type display. 
However, whatever compensatory characteristics remaining in the display also help to maintain congruence, by means of maintaining the proximity of movement stereotype.

In general, pursuit displays permit the observer to distinguish between cursor motion and target motion, whereas compensatory displays confound the two and present only the relative error between the cursor and the target. What happens, however, when the information provided by a pursuit display is no different from that derived from a compensatory display? One example of such a case is when tracking is required at very low velocities (so low that displayed motion is near or below the threshold of human perception). In this case there is no difference between the information communicated by either pursuit or compensatory displays, since little or no motion information is transmitted anyway. Only reactions to minimally discriminable or just noticeable differences between the cursor and target will result. In that case, the tethered display format may as well be $100 \%$ compensatory-like (pursuit type II), since all necessary cursor-target information would still be provided, but the probability of control reversals would more likely be reduced. Thus, using a compensatory mode has no disadvantages, but, as outlined above, may have distinct advantages.

Graham (1965, as cited in section 5.201 of Boff and Lincoln, 1988; and by Regan, in chapter 7 of Salvendy, 1997) and others have conducted early research into the detection of real motion parallel to the frontal plane. The detection of real motion contained in the fronto-parallel plane has been reported to have an absolute threshold of roughly 1 to 2 are min of visual angle/sec when the observer fixates the moving target, and provided that 
there are one or more stationary reference marks close to the moving target. This minimal threshold of detection is similar to that of detecting motion between two targets (cf. section 5.1.4). However, when the target moves against a textureless background (i.e. where all stationary reference marks are removed) or dark background, this threshold rises by an order of magnitude, to $10-20 \mathrm{~min}$ arc/sec (Leibowitz, 1955, as cited by Boff and Lincoln, 1988).

The lower of the two thresholds cited is associated with the detection of object-relative motion information that is available from relevant changes in the position of retinal images of objects. Both compensatory and pursuit displays permit the perception of this type of motion. However, the higher threshold cited is associated with the detection of subject-relative motion, that is, information about any single target in absolute terms relative to the observer. This information is extracted from both retinal-image motion and information about the movement of the observer's own head and eyes (Section 5.201 in Boff and Lincoln, 1988). Only pursuit-type displays allow the operator to take full advantage of this increased amount of information, since both target and cursor motions may be perceived. Compensatory-type displays, on the other hand, confound this characteristic of subject-relative motion by presenting only changes in the positions of both objects relative to the observer, thereby hindering tracking performance. When object-relative information is the only information available (e.g. for low frequency motions), it reliably indicates that one or more objects are moving, but does not specify a zero velocity point. In this case, information about the object motion is ambiguous in any case and applying a compensatory-type display would not harm tracking performance. 


\subsubsection{Some Examples of Dynamic Tethering}

Several third-person ${ }^{13}$ video game designs have implemented techniques that mimic the functionality of the dynamic tether. A couple of examples include: $X$-plane by Laminar Research (http://www.x-plane.com), which uses a translationally-slaved, fixed attitude tether, and Core Design Ltd.'s Tomb Raider III, which uses a translationally-slaved, heading-slaved tether (Wickens, 1998). X-plane makes use of a rigid tether, where the viewpoint is uncoupled, however, from the rotational dynamics of the simulated aeroplane. The operator is given a view that remains a constant distance from the aeroplane avatar, but is allowed to view changes in orientation by portraying changes in aeroplane heading rather than world rotation. Tomb Raider III, on the other hand, provides a functional tether in all 6 degrees of freedom (DOF). The developers have used quatemion calculations to animate all camera movements ${ }^{14}$ (www.gamasutra.com/ features/programming/19980703/quatemions_01.htm, July 3, 1998). It is commonly believed that commercial game designers employ heuristic rules in their designs, rather than thoroughly researched design guidelines, and thus it is likely that the particular tethering algorithms cited here have been designed to handle only the frequencies in their particular game spaces.

\subsubsection{Design Trade-offs: Changing the Tether's Dynamic Response Characteristics}

The frequency response of the dynamic tether is a function of two degrees of freedom, i.e., $\zeta$ and $\omega_{n}$. Decreasing the damping ratio, $\zeta$, below 0.7 will have an adverse affect on 
the system when forcing function frequencies exceed the natural frequency of the tether, $\omega_{n}$. Although decreasing $\zeta$ will allow the viewpoint to reach initial alignment with the operator's controls more quickly, high frequency inputs will cause the viewpoint to oscillate about the aligned vector. Such oscillations about the aligned vector would be undesirable, would likely make accurate control very difficult, and might even cause the user to feel physically ill at ease. Thus $\zeta$ should be kept above 0.7 , preferably about 1.0 . At values of $\zeta$ greater than 1.0, excessive time is required for viewpoint alignment to be achieved. A damping ratio of 1.0 was used throughout the present study.

The natural frequency, $\omega_{n}$, of the tether defines the nominal breakpoint frequency, as well as the stiffness $(K)$ of the tether, since $\omega_{n}=(K / M)^{1 / 2}$, and (hypothetical) mass $(M)$ is held constant in this study. Thus, the reader may choose to think of the discussion in this study in terms of stiffness of tethers, which vary proportionally with the square of the natural frequency. Frequency components of the input control signal with values less than the natural frequency will not induce much motion in the cursor, but will instead add most of the cursor input to the target disturbance signal. Those frequency components above the natural frequency of the tether, on the other hand, will manifest themselves mostly in the form of cursor movement, with some small fraction of the input added to the target disturbance signal. This fraction approaches 0 as the input frequency approaches infinity and approaches 1 as the frequency approaches zero.

For example, for input control at a frequency $\mathbf{X}$ which is below the specified natural frequency, the background of the display will move a displacement of $Y$; for a second 
input at approximately the natural frequency, the background may move a displacement of $-0.5^{*} \mathrm{~W}$ and the cursor may move $0.5^{*} \mathrm{~W}$; and finally, for an input control frequency $\mathrm{V}$ which is well above the natural frequency the cursor may move a displacement of $Z$ against a stationary background. If the natural frequency of the tether is set too high, then most of the motion due to operator input will be added to the target disturbance, and the tracking task will become relatively difficult. If the natural frequency is set at too low a value, on the other hand, then the cursor will be allowed to venture into configurations that violate control-display congruence principles, and again performance is likely to suffer. One of the goals of this study is to obtain a rule for estimating values of break frequency, in order to ensure an optimal combination of the two display modes.

12 Type II here refers to the fact that the author believes that this type of pursuit display has more compensatory-like chanacteristics than the traditional (Type I) pursuit display described above.

13 "Third-person video games" refer to games in which users have a bird's-eye-view of their actors or avatars (i.e., the representation of themselves within the virtual world), in contrast to games in which the user display provides a first-person, egocentric view of the game space through the eyes or the window of the avatar.

14 The main advantage of using quaternions is that it is computationally less expensive to add angular velocities to quaternions than to matrices. In the current study matrix calculations have been used on the experimental platform. Future studies, however, may consider using quarternion calculations to improve the smoothness in the real-time scene rendering, which may or may not affect subjects' tracking performance. 


\section{Performance Measures}

\subsection{Tracking a Quasi-random Sine Wave Track}

In order to investigate the principles discussed thus far, a tracking experiment was carried out using a quasi-random (sum of sine waves) forcing function. One of the problems with such tracking functions, however, is that the large amplitude, high frequency components within it tend to determine the average frequency of reversals in the response (Poulton, 1974). In other words, operators tend to predict reversals at the highest perceptible track frequencies. As a consequence of this phenomenon, the amplitudes of the components of the quasi-random track used in this experiment were decreased with increasing frequency. The resulting inverse relationship between amplitude and frequency ensures that the top frequencies do not dominate the other frequencies in the track, and the operator should not attempt to track only the high frequencies.

The track generation algorithm used in this study is similar to that used by Zhai (1995) to produce a quasi-random sinusoidal track (see equation 7-1). This method was also used by Macedo et. al (1998). However, the coefficients used here to decrease the component amplitudes as the component frequencies increased were modified from those used by Zhai, because the present experiment required specific frequency-amplitude pairings, to ensure that particular output velocities would be present in the target track. In particular, the velocities that drove the modification of the formula were those derived from the motion perception thresholds described in sections 5.1.4 and 5.2.3. 
When a forcing function is composed of many sine waves, the intention is that the subject perceive the tracking signal as being essentially random (hence the term "quasirandom"). As a consequence, subjects may respond at any frequency, and thus the frequencies in the output do not necessarily correspond perfectly with those of the forcing function input. As the highest input frequency is increased, the match between the track and the response decreases. A closed loop transfer function can be used to illustrate the closeness in this match between the track and the response, in terms of RMS amplitude and relative phase lag. Such transfer functions summarise the human's predictions and pre-programmed responses in terms of how successful they were in reproducing the track input. In terms of the present study, if using the dynamic tether indeed improves the human's ability to track forcing function inputs, then we would expect to see a high correlation between the tracking input and the human's response output. Any improvements in performance may occur in three ways. First, the relative RMS error amplitude may decrease; or second, the overall input-output phase lag may be reduced; or, third, a combined improvement in terms of both RMS error and phase lag reduction may be achieved.

In a simple choice reaction time experiment, in which the visual signal is expected, one would expect to observe an average time lag of 130 to 170 milliseconds (Poulton, 1974; Wickens, 1990). Phase lags that are produced by smaller time delay values would thus indicate that some prediction of the stimulus was successfully taking place. Conversely, greater phase lags would indicate that, for some reason, prediction is less accurate, or 
may be present in a reduced capacity. For example, increasing the mental workload of a subject might conceivably increase the phase lag, and vice versa.

Poulton (1974) pioneered much of the work in tracking and manual control, and his work on tracking quasi-random sine wave functions is of particular interest for the present study. Initial estimates for the tracking frequencies used here were extracted from Poulton's publications. Once the required retinal velocities of motion were calculated, the frequencies and their corresponding track amplitudes were determined by trial and error, in order to set an appropriate level of task difficulty. Two factors were taken into account to govern the difficulty of the task. First, it was reasoned that increasing or reducing the amplitude of the forcing function might respectively increase or decrease the precision of the operator in the tracking task. That is, since changes in low amplitude signals are difficult to perceive, the ability to correct them deteriorates with decreasing magnitude. The track amplitudes were therefore designed to be inversely proportional to the track frequency, with the highest frequency chosen so that its associated amplitude was not so low as to cause unreasonable relative errors in tracking. Second, increasing track frequency alone increases the degree of tracking error. Poulton's studies have shown that performance begins to suffer when the top frequencies in the quasirandom track exceed 40 to 60 cycles per minute (cpm). In this experiment the top frequency used was approximately $50 \mathrm{cpm}$. As Poulton states: "Very high frequencies of large amplitude make tracking virtually impossible, and have to be excluded." 


\subsection{Measures of Periormance for Single Step Tracking}

Single step tracks (such as those presented in a docking task) comprise an isolated, sudden change of position. The jumps are unpredictable (due to randomisation of docking presentation instants) and the operator is required to elicit a rapid appropriate control response to bring the cursor into the new desired position. Such quick step input changes in position correspond to a high frequency content. Recalling that human operators have a total processing bandwidth of about $1 \mathrm{~Hz}$, which makes it very difficult to follow high frequency components accurately, it is not surprising that tracking performance suffers with step inputs.

In addition to the dynamics of the system being tracked, single step track performance depends on reaction time, the amplitude of control movements, the accuracy of the response, and response pacing demands of the track. When the time to reach the target is limited and the operator makes large, quick movements, he may not be able to respond quickly enough to the rapid approach of the target and may overshoot it. When a target's jump is unexpected, or if the operator does not know which of many jumps will occur, then the control response will not begin until at least one reaction time after the signal (jump) is presented. The extent of the total reaction-time lag depends on numerous factors that can be related to the information-processing characteristics of the operator. For example, when the operator does not know which control response will be required, the decision-making stage introduces additional increments to the total reaction time delay. 
The size of required control movements also affects the accuracy of response when movements are quick. Within the context of a docking task Craik's Ratio Rule (Poulton, 1974) states that accuracy does not suffer for required cursor-to-target movements that are greater than $\mathbf{2} \mathrm{cm}$. The rule states that the ratio of average absolute error to distance is somewhat constant (approximately 0.05 ). It is also known that the time required in making an accurate movement over a significant distance to a target increases as the distance increases and is governed by Fitts' Law (the Index of Movement Difficulty). Fitts' Law also indicates that placing a time constraint on the movement can affect task execution and decrease accuracy, resulting in under- or over-shooting of the target. Also, Craik's Ratio does not remain constant for very small distances (i.e. $<2 \mathrm{~cm}$ ). In fact the ratio of average absolute error to distance increases rapidly as distance decreases. This reflects the impact of physiological noise (i.e. muscle tremor) from the response-control stage of the operator information-processing model.

In addition to considering the absolute size of control movements in the present project, we are also interested in whether a relationship exists between the kind of mental rotations studied by Cooper and Shepard (1973) and the mental operations required in performing an angular displacement docking task given a pursuit (type I) display. The docking experiments reported here are designed to investigate this question and several others. In particular, it is expected that the time to react may be proportional to the size of the displacement between the target and the cursor. As well, a possibly more significant effect on reaction time may be due to the angular departure of the cursor itself from its canonical upward/forward facing orientation. Shepard and Hurwitz's (1984) findings lead 
us to believe that the direction of departure from the vertical is not of relevance, but only the magnitude of the departure will affect the time to discriminate turn direction. That is, as the magnitude of departure increases, reaction times typically increase more sharply as the departure from upright approaches $180^{\circ}$. However, as departures approach $180^{\circ}$ more or less, we would expect to see a drop in reaction time as a reversal strategy is adopted.

\subsection{Measures of Performance for Dynamic Tracking}

Tracking ramp inputs of constant velocity is apparently slightly easier as a visual task with a compensatory display presentation than with a pursuit display (Poulton, 1974). The advantage, however, is too small to produce a substantial difference in performance. This is due to the fact that detecting small gaps between moving markers using dynamic visual acuity, as with pursuit displays, is more difficult than for compensatory displays, where detecting small gaps between a stationary marker and a nearby error marker more closely resembles static visual acuity. Therefore, provided that the operator tracks reasonably accurately with the compensatory display, the visual task should be slightly easier than with pursuit. Whereas ordinarily the operator cannot easily perceive the velocity of a tracking error with a compensatory display, this may not matter much with a ramp track of constant velocity since the rate of the tracking signal does not change in any case. However, if the background is included in the scene, then the possibility of inducing motion in the stationary markers exists and some of the disadvantages of the compensatory display may be eliminated. 
Tracking is more difficult with irregular tracking inputs containing relatively high frequencies, because track prediction becomes more difficult.

\subsubsection{Measures of Performance for Sinusoidal Tracking}

In order to analyse how human beings track signals of a continuous, periodic or random nature, the inputs should be represented in the frequency domain. The operator's control response may then be broken down into its component frequencies to determine what proportion of the response was composed of frequencies that were present in the target track, and what proportion is uncorrelated with the target track. Also, the amplitude of the output response may be analysed at each frequency, to determine how closely the operator control response frequencies mimic the forcing function frequencies. Additional analysis of time or phase lags (discussed in section 6.1) may also be performed.

Fourier analysis breaks the signal down into a set of constituent frequencies (Wickens, 1986). Each frequency component, at a particular frequency $\omega$, in radians per second or hertz, is specified by its amplitude, $A$, and its phase angle, $\theta$. Frequency-domain signals are typically represented graphically as power spectral density functions, which preserve amplitude and phase information at each frequency. For illustrative purposes all power spectra will be plotted here as a continuous function across the range of frequencies in the signal, instead of the series of discrete spikes which represent estimates at the discrete frequencies computed. 
The closed-loop transfer function reveals the proportion of each frequency in the input tracking signal that appears in the output response of the system. As the human operator makes changes in his strategy and the dynamics of his response, thereby changing the control signal frequency, he affects the closed-loop transfer function in terms of its average amplitude, the time lag in the responses, and the size of the remnant. The remnant is defined as the portion of the operator's output response spectrum that is not present in the input signal. The size of the remnant increases with increasing input signal frequency. In this study, it is the difference between the output response spectrum and the input signal spectrum that is analysed (i.e. the error signal).

\subsubsection{Averaged Measures of Error}

There are several measures that may be used in tracking to reflect average error, that is, the overall average distance between the cursor and the target:

(1) Constant position error is the arithmetic mean of the distance error and indicates the extent to which the average position is above or below the track. However, because both positive and negative values are possible, the signed errors in the calculation tend to cancel one another.

(2) A standard deviation of the error distribution (variable error) may be calculated, by summing the squared differences between the constant position error and each individual error.

(3) Average absolute error, or "modulus mean error", is the arithmetic average of the unsigned (i.e. absolute) momentary position errors, reflecting both variable error and 
constant position error. This measure overcomes the positive-negative cancelling problem with constant position error (1).

(4) Lastly, the root-mean-squared (RMS) error is the square root of the sum of the momentary errors, each squared, and finally averaged. RMS errors can be calculated precisely from a record of a given length and individual RMS measures may be combined easily. Thus the total error in a system containing several operators can be determined by squaring and summing the component RMS errors, then taking the square root of the total. RMS error is the ideal measure for this study due to the number of experimental trials and operators involved. 


\section{Justification and Hypotheses for a Novel Study}

Teleoperation and other applications are making use of virtual and mixed reality to an increasingly greater extent. These "alternative" display formats have many advantages over traditional media. 'Virtualising' (or modelling) the task space allows humans to interact with environmental information in ways that were never before possible. Viewpoint manipulation is one of the capabilities provided by this relatively new technology, where both manual viewpoint manipulation and animated viewpoint narration have been applied to create paradigms that were, until recently, possible only in the world of computer entertainment. Many of the teleoperation scenarios discussed in previous sections provided both planar viewpoints (e.g. the attitude-direction indicator) and perspective viewpoints (e.g. Das's 1989 study); therefore it makes sense to investigate the effect of viewpoint on tethered performance. It is assumed that there will be no significant difference in any performance measures with respect to the given viewpoint.

Although many have studied such manipulations in the medical and aviation fields, relatively little is known about applying similar solutions in space, land or sea environments. Furthermore, in partially modelled environments, such as those which use augmented-reality displays, the problem of control-display incongruency is prevalent (Milgram and Colquhoun, 1999a and 1999b). For performing the kind of variable viewpoint telemanipulation of interest to this project, two major problems emerge from a review of the literature: (1) operator mental workload typically increases as the misalignment between control and display grows, and (2) coupling the (camera) 
viewpoint to a controlled object to take advantages of the scaling properties of such a display may reduce the amount of motion information available to the operator, thereby potentially diminishing operator performance. The latter is especially true if a discernible background structure is not present.

\begin{abstract}
Although studies have been performed elsewhere to investigate such related factors as the use of rotating maps for navigation, the application of frequency separation techniques in aviation, the effects of mental transformations on image rotation, etc., none of the results generated there may be applied directly to telemanipulation tasks without further in-depth research. In particular, the application of frequency-separation outside the field of aviation is lacking research, which is significant because applying this technique may be one possible solution to many of the problems mentioned above.
\end{abstract}

The present study represents a first attempt to answer the question of whether or not the concepts of tethering and frequency separation can be combined in the design of displays for telemanipulation and navigation to assist the human operator in better performing tracking tasks. Towards this end, a combined telemanipulation docking-tracking task was designed and several performance measures were collected and analysed. These included time-to-completion, overshoot, control reversals, and reaction time for the step input docking-type tasks. Sinusoidal tracking performance measures for the dynamic tracking portions of the experiment included overall root mean square (RMS) error and RMS error as a function of input track frequency. 
It was hypothesised that dynamic tethering will improve performance on some measures while obtaining at least the same measure of performance on others. More precisely, as tether natural frequency; the independent variable selected as a means of manipulating stiffiness, or rigidity, is changed along the continuum from true-motion displays $\left(\omega_{n}=0\right.$, or zero stiffness tether) to relative motion displays $\left(\omega_{n}=\infty\right.$, or infinitely rigid tether), we expect to obtain a generally U-shaped function when plotting performance against $\omega_{n}$. That is, on the one hand, the more pursuit-like displays $\left(\omega_{n}=0\right)$ should perform closer to optimal in dynamic tracking tasks, as they allow the operator to take advantage of perceiving both absolute and relative motion components of the display, as long as deviation from compatible control-display alignment is not significant. On the other hand, when incongruent alignments occur in this condition, we can expect performance to suffer. More compensatory-like displays $\left(\omega_{n}=\infty\right)$ would then benefit from their scaling characteristics when this is the case. However, as the pursuit-like characteristics of the display diminish, the advantages of dual motion perception are lost and performance is expected to be curbed again.

Having said all this, the following hypotheses are proposed.

Docking task hypotheses:

(1) Task completion time and target overshoot measures will increase (reflecting a decrease in performance) as the natural frequency of the dynamic tether is increased. It is assumed that the perception of system motion is critical in keeping these measures to a minimum. 
(2) It is postulated that, as the dynamic tether's natural frequency is increased, the compensatory-like properties of the display will be enhanced and the proportion of time that the display is in misalignment with the isomorphic control device used will decrease, thus decreasing the chance of misinterpretation and the probability of occurrence of control reversals.

(3) Also due to the expected decreased loading of mental processes in this case, reaction times are expected to improve with the mere presence of a tether ${ }^{15}$.

Tracking task hypotheses:

(1) It is postulated that overall RMS error will increase with increasing pursuit characteristics $\left(\omega_{n} \rightarrow 0\right)$ when the target track promotes control-display incongruency by incorporating varying frequencies of motion with high amplitude.

(2) It is postulated that RMS error will increase with a corresponding increase in compensatory-like characteristics $\left(\omega_{n} \rightarrow \infty\right)$ when the target track contains fairly high frequencies of varying amplitudes.

(3) We expect to observe a generally U-shaped function of RMS error versus natural frequency, since the appropriate combination of pursuit and compensatory characteristics will result in optimal performance somewhere between the extremes.

(4) As the natural frequency of the tether increases, we expect that performance at low frequencies will not differ significantly across tether values, but at higher track frequencies we expect to find the U-shaped function described in (3). 
is In the docking scenarios the initial conditions of the tethered system were constrained to be in a state of equilibrium, i.e., with no relative cursor-camera motions. Thus, all treatments in which a tether was present began with high control-display alignment, since by definition a tethered viewpoint must be egocentrically aligned under steady state conditions. This was not always the case when no tether was present, on the other hand, since alignment depended only upon the absolute value of the initial alignment angle. Controldisplay misalignments are thus caused by cursor displacements from a base vector while the magnitude of the required step input is the angular displacement between the cursor and the target. 


\section{Experimental Method}

This experiment was designed to discover how human operators perform when executing both docking and tracking tasks, in a completely virtual environment, with displays that are dynamically tethered to the on-screen representation of the objects being controlled. These experiments are a small step towards confirming the theory discussed in the previous chapters. It is not the investigator's intention to prove all of the concepts discussed. However, future follow-up research may create a more established theory of mixing pursuit and compensatory display information.

\subsection{Experiment Set-up}

\subsubsection{Experimental Platform and Apparatus}

A Silicon Graphics Impact graphics system was used to present the docking and tracking tasks on a high-resolution 17-inch monitor. The display refresh rate was $60 \mathrm{~Hz}$; however, because screen update rate fluctuated with computational load on the SGI Impact, the actual resulting update rate varied between 25 and $35 \mathrm{~Hz}$. The interface was designed such that it provided feedback and instructions to the user, and logged experimental data with little intervention by the experimenter.

The experimental set-up is illustrated in Figure 20. Subjects were seated in a chair with an adjustment for height. The height was set such that subjects were seated in front of the monitor with their forearm resting comfortably on the 4-degree of freedom (DOF) input device used in the task (Figure 19). This input device accepts input from forearm rotation in the frontal and sagittal planes, as well as from wrist rotation and forearm front-to-back 
translation. Only inputs from forearm rotation in the sagittal plane (1-DOF input) were used within this study; input signals from all other degrees of freedom were ignored by the program.

Subject eye-to-screen distance from the monitor was approximately $50 \mathrm{~cm}$ (Figure 20 (b)). The height of the subject's eye level relative to the monitor was not controlled, since it was more important that the subjects' seat height be adjusted such that their arm rested comfortably in the armrest of the controller both when static or in motion. This helped to ensure that fatigue would not set in due to inappropriate positioning of the subject's arm while tracking the on-screen target.

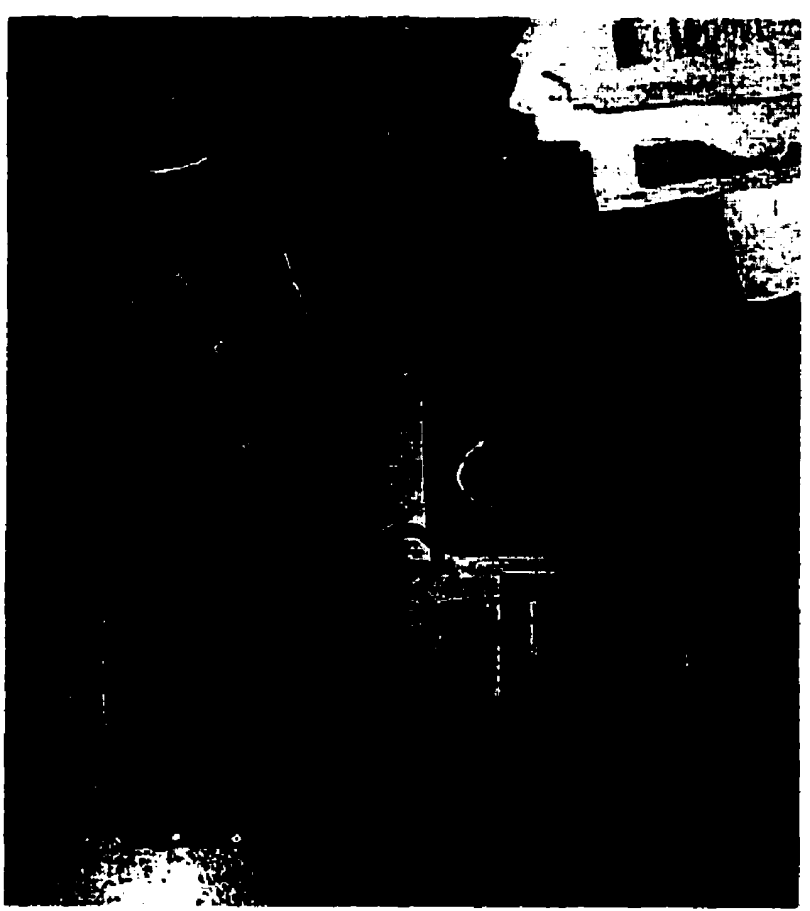

Figure 19 The 4-DOF joystick input device 


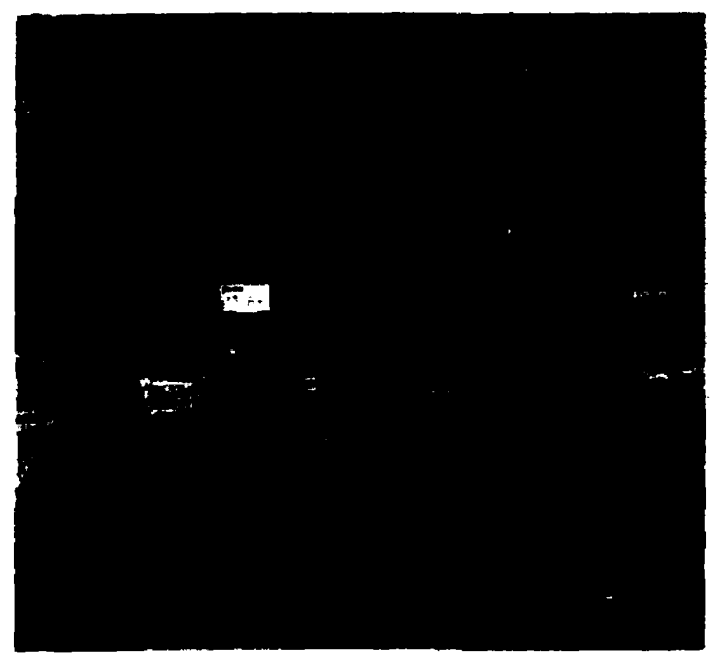

(a)

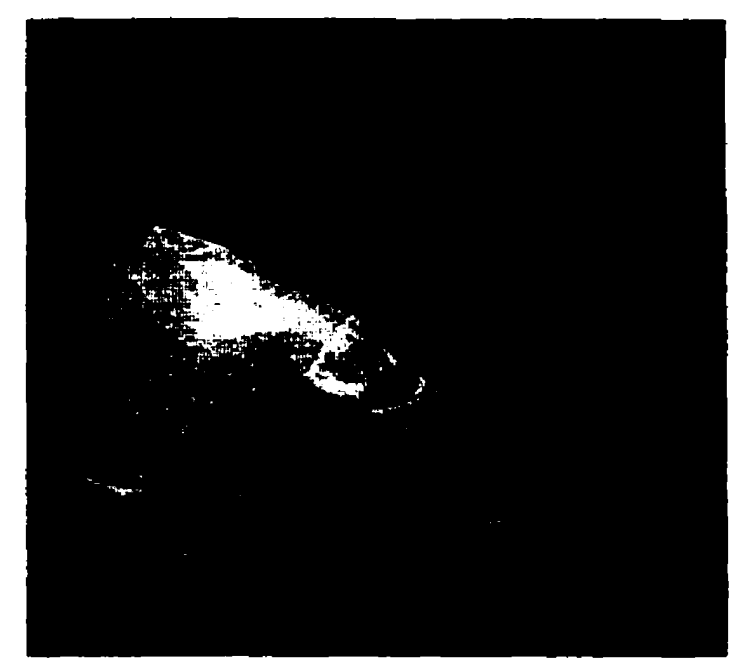

(b)

Figure 20 Experimental Set-up

\subsubsection{Experimental Displays and Tasks}

The objective of this experiment was to study the effect of dynamic tethering on 1 DOF control. The display was thus designed to provide cues that would ensure that task performance would be affected predominantly by differences in tethering parameters, rather than controller dynamics and display ambiguities. A range of five different tethers was examined, as explained below.

The task space consisted of a cursor, which was depicted as a "cannon" with a rotating turret, and a 3-dimensional arrow as a target. Illustrations of the cannon, target indicator, and background that constitute the display frame are shown in Figure 21. The background was a flat surface with a grid overlay, with the grid providing the structure for the background as required to enhance perception of absolute motion of the cursor and target. Initial pilot tests revealed that including the grid was necessary to distinguish movement 
of the base of the cannon (which was attached to the ground or world reference frame) from movement of the turret or target.

The origin of the virtual environment was collocated with the centre of rotation of both the target, the cursor, and the centre of the computer screen surface. All objects were drawn using perspective projection. However, this was only apparent when the "perspective" viewpoint condition was presented, since the "top-down" or plan view produced an orthogonal projection of the scene (Figure 21).

Frequency Separation: Choosing Values of $a_{h}$ and $\zeta$.

The 1 DOF tracking task comprised an operator moving the cursor following the target which was driven by a forcing function. In order to make the target path sufficiently unpredictable to the experimental subjects, the forcing function was derived from a weighted summation of 11 sine wave functions, given in Equation 8-1. The design was based on a sinusoidal power function used by Zhai and Milgram, 1994, where B (the amplitude of rotation), $\mathrm{q}, \mathrm{p}$, and $f_{0}$ (the base rotation frequency) were derived from the motion detection threshold limits for real and induced motion and by limiting the highest frequency to $50 \mathrm{cpm}$. The factors $q$ and $p$ were included, respectively, as a means for defining the value of the $i-$ th frequency component and for successively reducing its amplitude for reasons discussed in section 6.1 and Appendix D. The values used in our experiments were $B=2.26548$ radians, $q=1.20094, f_{0}=6.27226 \times 10^{-4} \mathrm{~Hz}$, and $p=$ 2.05372. $\$(i)$ was a random number representing the phase of the $i-t h$ component. 


$$
\theta(t)=\sum_{i=0}^{10} B q^{-i} \sin \left(2 \pi f_{0} p^{\prime} t+\phi(i)\right)
$$

The value of $p$ above was chosen such that the frequency components in the forcing function would be multiples of the natural frequency of the particular tether used. Recall that the natural frequency, $\omega_{n}=2 \pi f_{a} p^{i}$, of the dynamic tether determines the breakpoint in the frequency separation algorithm (i.e. the point that defines the midpoint of transition between compensatory-like and pursuit display modes for that particular tether). The first tether value reflected a connection between the "camera" and the "cannon" with the characteristics of a very loose tether, that is essentially no tether at all (i.e., $\omega_{n}=0$ ), which defines the $100 \%$ pursuit-compatible (type D) display. This condition is depicted as the bottom row of Figure 21. The last of the five tether values, shown along the top row of the figure, was a completely rigid tether (i.e., $\omega_{n}=\infty$ ) and defines the $100 \%$ compensatory-like (pursuit type II) display. The three intermediate frequencies divide the 11 frequencies of the forcing function into four groups. As explained below, the particular values used for the second and fourth tether values were based on human limits for motion detection, while the third tether value was calculated as the frequency defined by equation 8-1 at $i=5$. See Appendix $D$ for a detailed derivation and calculation of the frequencies used in the forcing function.

Referring to equation 8-1, at $i=2$, the frequency used for the forcing function was calculated based on: the subject's eye-to-screen distance $(50 \mathrm{~cm})$, the radius of the cursor's traced path $(7 \mathrm{~cm})$, and the theoretical lower human threshold for induced motion perception - 1-2 min arc of visual angle/sec (Section 5.201 in Boff and Lincoln, 
1988). Creating a tether with a natural frequency corresponding to this value would result in camera animation that responds to input frequencies close to and below the $i_{2}$ frequency. As discussed in Section 5.1.4, the author is particularly interested in this $i_{2}$ breakpoint frequency because, in theory, induced motion does not exist below this value. (This means that subjects should not be able to derive induced cursor motion from camera rotation; instead any such motion should be perceived as true world rotation.) Without the induced motion effect there is less value in rotating the camera at low frequencies because the motion with respect to either reference frame (ego- or exocentric, as per compensatory and pursuit displays respectively) is not likely to affect task performance. That is, if no motion of the cursor is induced at low frequencies then world rotation will not promote tracking performance, and in fact it may hinder it. Such world rotation is a product of pursuit type II displays, which occur with very rigid tethers, and if world rotation hinders performance more than the preservation of control-display congruence supports performance, then theoretically there is no added value to using such a display tether over the traditional pursuit (type I) display. In more specific terms, the $i_{2}$ frequency was included in order to determine whether non-rigid tethers would outperform very rigid tethers in the vicinity of this frequency, as expected. ${ }^{16}$

\footnotetext{
${ }^{16}$ Real motion cannot be perceived at visual angular velocities less than 25 seconds of arc/second. It does, however, make sense to present the information in a more compensatory manner at these low frequencies ( $<2$ minutes of are per second) to maintain control-display congruency, since the "true motion" pursuit display advantages would not be present at these frequencies anyway.
} 
The fourth tether's natural frequency (corresponding to forcing function component $i=8$ ) was based on a frequency of $50 \mathrm{~min}$ arc/sec, the upper threshold for induced motion. Using a forcing function equation with the two aforementioned ( $i=2$ and 8 ) natural frequencies, with corresponding amplitudes of $\pi / 2$ and $\pi / 6$ respectively, the parameter values of Equation 7-1 were calculated. The natural frequency value of the third dynamic tether was then calculated at an intermediate value of $i=5$.

The five tether natural frequencies used in this experiment were thus, 1) $0.000 \mathrm{~Hz}$, 2) $0.002645 \mathrm{~Hz}$, 3) $0.022916 \mathrm{~Hz}$, 4) $0.19850 \mathrm{~Hz}$, and 5) $\infty \mathrm{Hz}$. A constant damping ratio of $\zeta=1.0$, which defines critical damping, was used for all tether values. In general, critically damped or overdamped tethers are required because, as discussed earlier, underdamping would result in unwanted oscillations in the camera animation (especially at the natural or resonant frequency). Critical damping ensures the approximate shortest possible transition time to a steady state view consistent with motion compatibility principles while preventing any transient oscillations. 

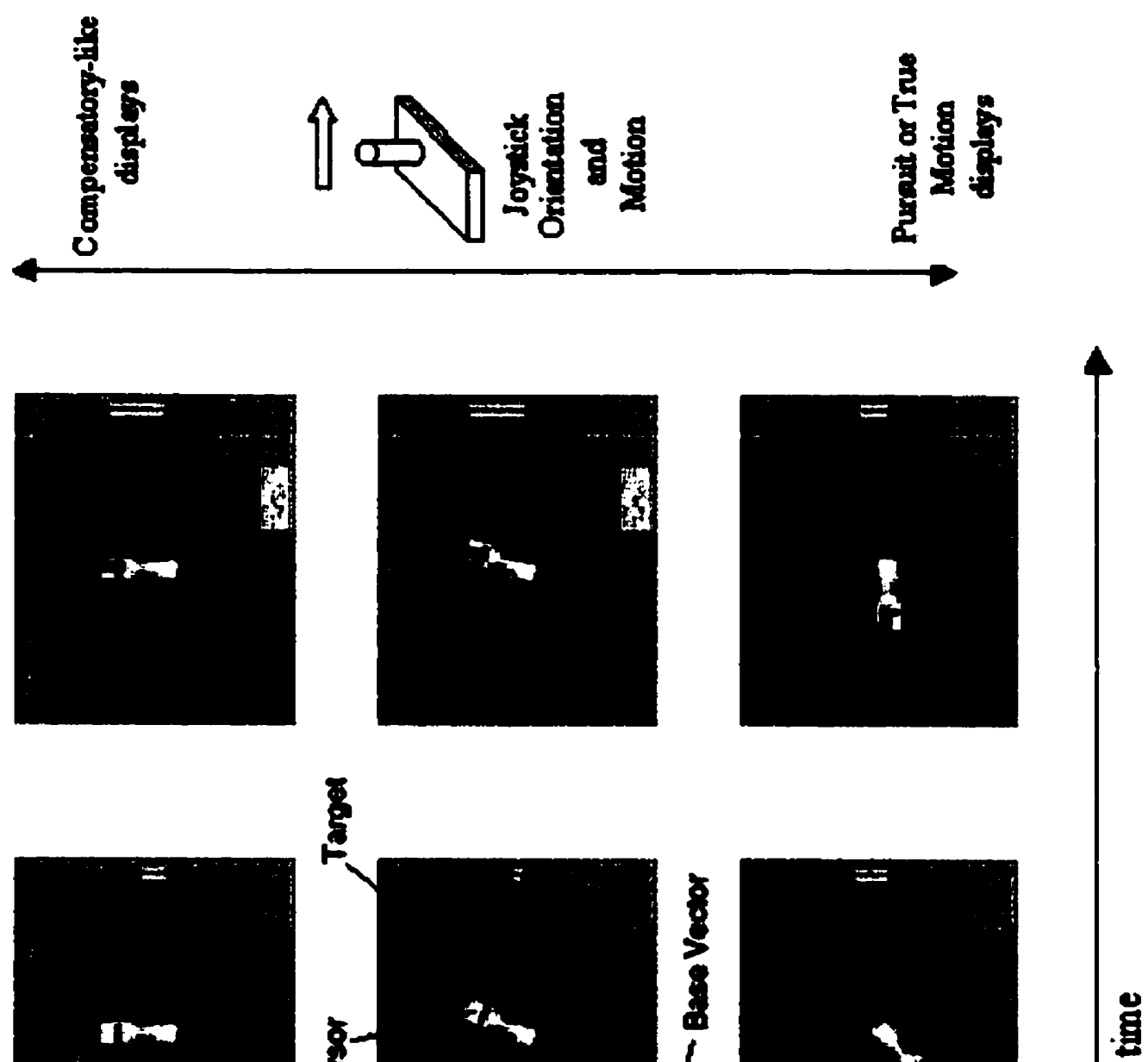

范需霖

은 롤일 읭 혈 응홍 옹 절홍응 这
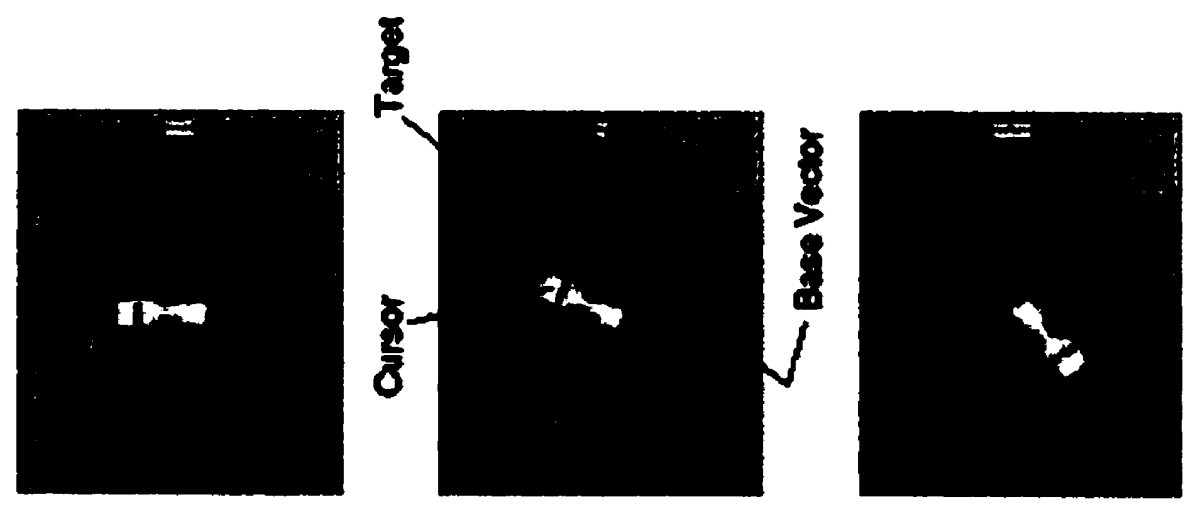
공

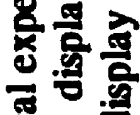
쿵요 롤흠 흘 8 홍 홀 졸 O 롱용 永泀 능 통 올을 음
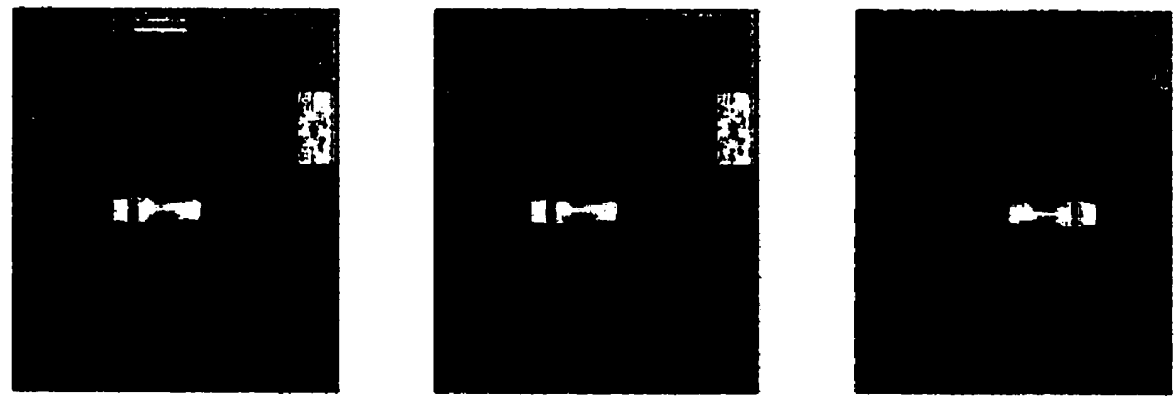

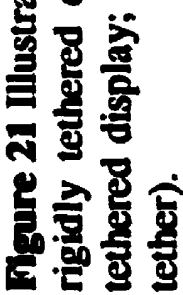

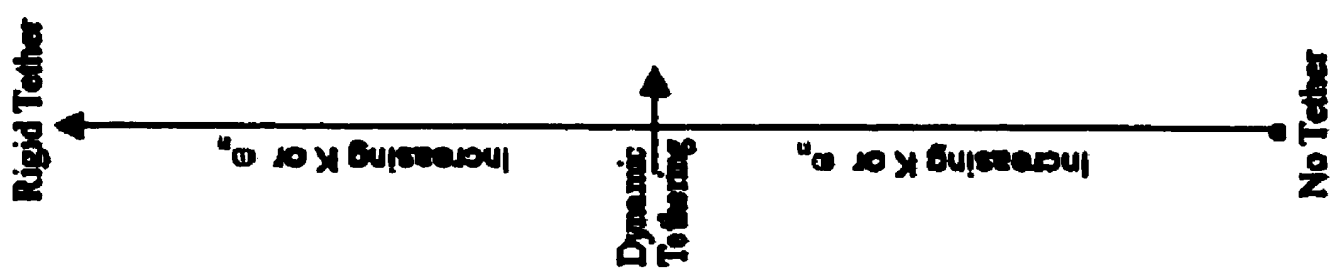


Experimental Tasks.

Subjects were required to perform a set of both docking and true tracking tasks, which were presented to them in a continuous sequence of presentations. A docking task consisted of the operator having to bring the cursor into alignment with the stationary target indicator using the joystick. A true tracking task required the subject to follow the moving target indicator as closely as possible. Each subject was to perform these tasks given various display characteristics defined by the five tethers described above.

\section{Task Performance Measures.}

There were eight measures of interest in this experiment. In the docking experiment control-reversal errors, percent overshoot, reaction times and completion times were computed. In the tracking experiment both overall Root Mean Square (RMS) errors for each tether setting and the RMS error at each input frequency (calculated via spectral analysis) were evaluated.

All measures except the frequency-based RMS error were calculated on-line during experiment execution. A control reversal was counted in a docking task whenever the initial motion of the operator's input resulted in an immediate increase in cursor-target displacement - i.e. a motion in the "wrong" direction. Reaction and completion times were straightforward measures taken from the real-time clock on the computer. Percent overshoot and RMS error were calculated as follows: 
Percent Overshoot $=\frac{\mid \max (\text { cursonangleswept)-initialcursorto targetdisplacemet }}{\mid \text { initialcursorto targetdisplacemat }} \times 100 \%$

(Equation 8-2)

$$
\text { RMS Error }=\sqrt{\frac{\sum_{i=0}^{N}\left(\theta_{\text {urpet }}-\theta_{\text {cursor }}\right)^{2}}{N}}
$$

where $\theta$ is the angle of either target or cursor, $i$ is the sample index and $N$ is the total number of sampling intervals.

\subsubsection{Optimisation of Control Gains}

In order to ensure that we are comparing different display designs fairly, we must ensure that the control-display relationship in terms of input-output ratios (i.e., the control gain) is optimal for each experimental condition. This way, we can be sure that we are comparing displays at the point of highest potential performance for each display (Zhai, 1995). It should not be forgotten that gain is not the only parameter that contributes to the optimisation of performance within an interface; however, as far as we are concerned, we shall consider gain as one of the most relevant parameters that affect manual control tasks. Thus, it is assumed that approximate optimisation of each interface may adequately be performed by optimising the gain for each display.

Manipulating system gains results in two general observations (Wickens, 1986): (1) The human operator can adjust his gain upward or downward to compensate for shifts in system gain (c.f. the cross-over model) in order to keep the total closed loop gain 
constant. This is valid across a wide range of values (i.e., of a 10-fold magnitude). However, (2) this compensation does not come without some cost and thus there is an optimal gain within this range (Gibbs, 1962). The optimal gain for static or dynamic target acquisition is a compromise between functions that affect the initial acquisition phase of the task and the final stabilisation required to hold the target in range (Wickens, 1986).

Given a high gain, initial target acquisition comes quickly; however, as the target is approached, oscillations may result due to the magnification of small corrections, and stabilising time may thus be prolonged. On the other hand, if the gain is too small, then the initial target acquisition time will be large, since larger movements will be required to approach the target. This trade-off results in a U-shaped gain function, for both position and velocity control systems (Gibbs, 1962). Frequently the optimal region of this Ushaped function is fairly large and optimal performance can be obtained across a range of gain values (Zhai, 1995). In addition, the system dynamics can affect the shape of this curve and the location of the region of optimal gain. System lag is one such system parameter that tends to shift the optimal gains higher.

In general, lower gains tend to facilitate better performance, but to a limit. Once this limit is reached and passed, lower gains tend to cause a rapid deterioration in performance resulting in a sharp turn in the performance curve. Low-gain systems will also introduce problems when dynamically tracking random input paths, because of the continuous and repeated high amplitude movements that the operator must produce. Conversely, for high 
gains, as precision requirements increase, the likelihood of instability also increases. Steady-state tracking data indicate that the optimum gain values are related to the tradeoff of physical operator effort at low gains versus the threat of instability and or the need for lead generation at high gain values (Wickens, 1986). In conclusion, we can thus expect to see optimal tracking occurring with higher gains for random input dynamic tracking tasks and lower gains for more static docking tasks.

In this study it was found that performance of an expert system controller was indeed a function of control gain or sensitivity. Figure 22 and Figure 23 illustrate completion time and percent overshoot data and RMS error data for that expert controller as a function of control sensitivity (gain), plotted on a logarithmic scale, for docking and tracking trials respectively. The functions are plotted for three dynamic tethers labelled w00, w02, and w99, which represent the first, third and fifth tethers discussed above, having natural frequencies of $0.0 \mathrm{~Hz}, 0.022916 \mathrm{~Hz}$, and $\infty \mathrm{Hz}$ respectively. The two remaining intermediate tether values, w01 and w03, were not included in the optimisation tests since it was assumed that the optimal region of these displays would lie between the optimal regions discovered for tethers w00, w02, and w03. 


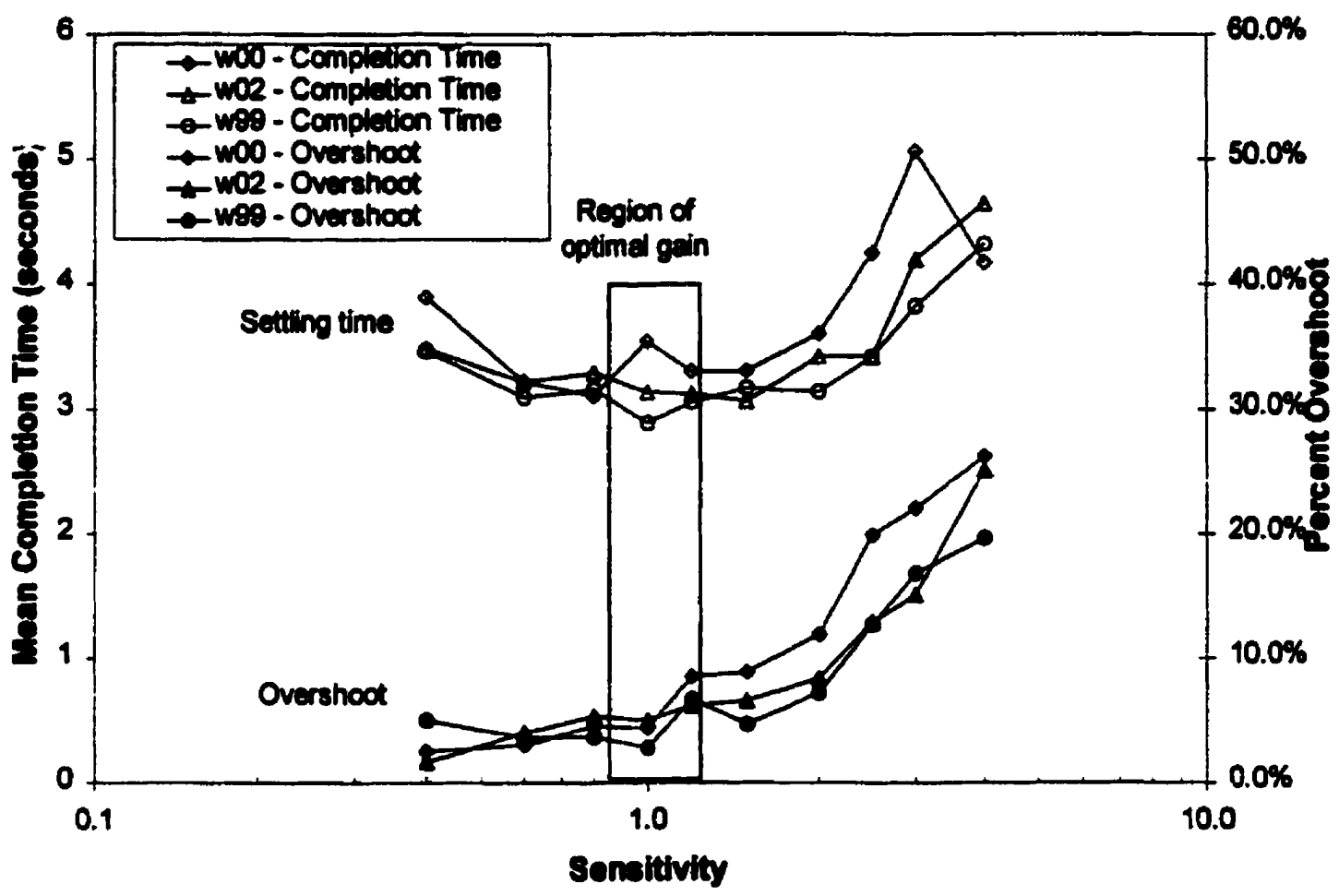

Figure 22 Optimisation of Sensitivity of the Tethered Display for the Docking Task

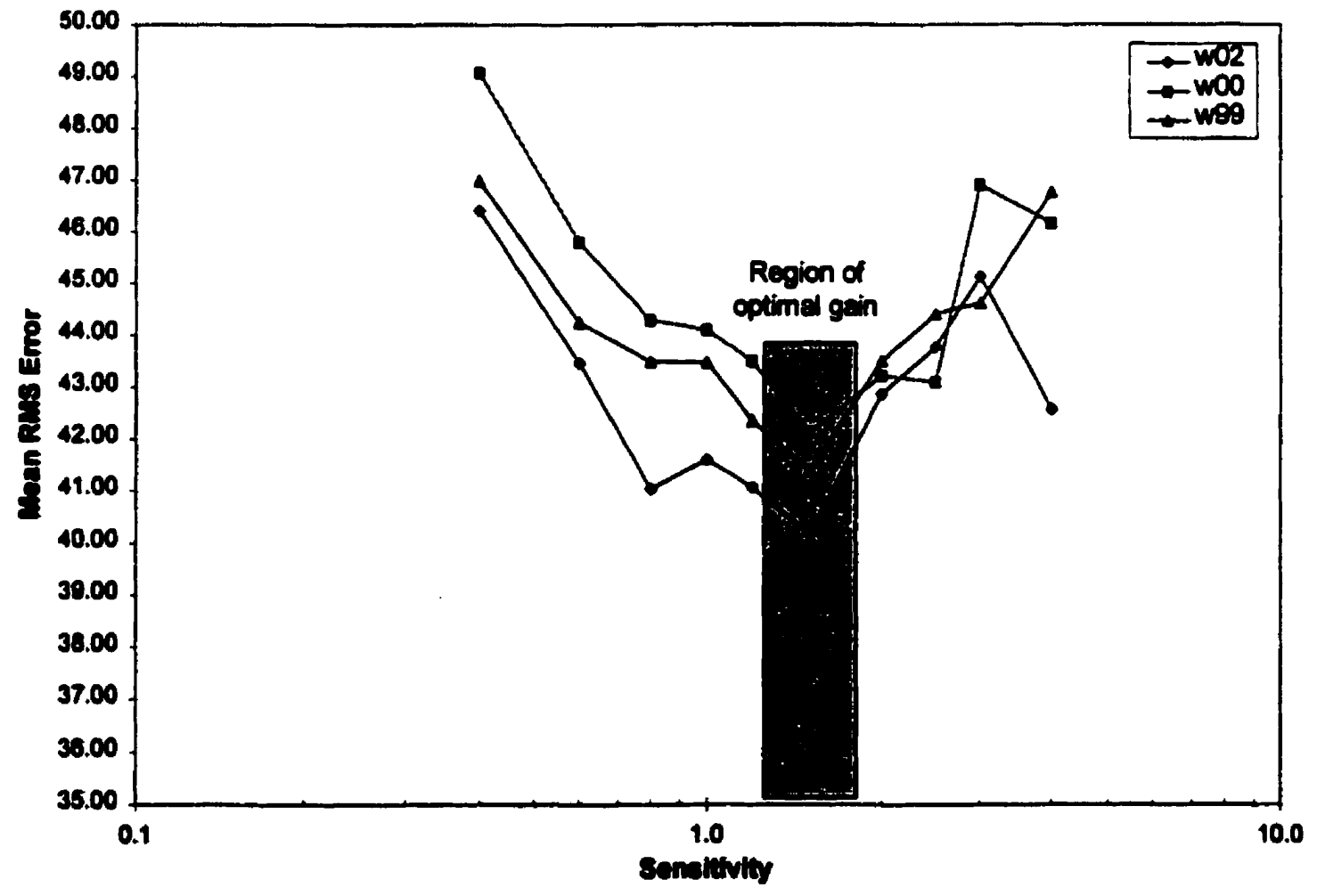

Figure 23 Optimisation of Sensitivity of the Tethered Displays for the Tracking Task 
The plots of completion time for the docking task (Figure 22) and RMS error for the tracking task (Figure 23) show that these performance measures did in fact produce the expected U-shaped function as control sensitivity is increased. The plots of percent overshoot in the docking task (Figure 22), however, produced a J-shaped function instead. This is because, as the gain of the controller increases, the ability of the operator to stabilise the cursor as it approaches the target deteriorates and thus the probability of overshooting the static target increases. Recall that low control gains increase the time required to acquire a target. This controller "lag" decreases performance on measures that contain temporal components, such as completion time and RMS error. Percent overshoot, on the other hand, is not dependent on time and thus control latencies do not affect this measure. Therefore, at low control gain settings, percent overshoot does not degrade.

There is therefore a value of control sensitivity below which target overshoot will not be significant and practically perfect performance on this measure will be obtained. This value of gain is much lower than the set of values of interest in the optimal region of Figure 22. Therefore, in choosing an optimal value of gain to use with each display for the docking task, a value of gain toward the left of the "optimal region" was selected. A gain of 1.0 was consequently chosen as the optimal value for the docking tasks. Similarly, an optimal gain value of 1.5 was selected from the range of values contained in the "valley" of the plots of Figure 23 for the dynamic tracking tasks of the experiment 
Finally, we note that, following similar earlier work by Zhai (1995), it was assumed that these same optimal gain values should hold for novice users performing the same experimental tasks. That is, even though they would be expected to generate lower absolute performance scores, they would nevertheless be subject to the same gain change factors as expert subjects.

\subsection{Experimental Procedure}

A tele-tracking task was created using the IRIS Performer 2.1 3D rendering toolkit, to study and enable control over modelled entities and the viewpoint through which they were observed. To study the effects of implementing the various types of dynamic tethers, a teleoperation scenario was provided to the subjects to put the task into context and to motivate their participation.

The subjects were told that they were in control of five cannons surrounding the perimeter of a fortress. The subjects' task was to ensure that the cannons were manually aligned with a large (3D) arrow that indicated the direction in which the cannon was to be pointed. In terms of the earlier discussion, the cannon represented the subject controlled cursor, while the arrow direction represented the target. For the docking tasks the cannons were to be aligned as quickly as possible with the arrow, while for the tracking tasks the cannons were to follow the arrow as closely as possible. This scenario was contrived in order to stress the importance of operator care and attention in performing the task, as well as to give the tasks a context, so that the participants would appreciate the task objectives. This scenario also facilitated the collection of both docking and 
tracking data within a single trial block. Five different dynamic tethers and their effects on various measures of docking and tracking performance were recorded.

\subsubsection{Experimental Design}

Five sets of experiments were carried out with each subject - one experiment per subject per day. Each set of experiments consisted of two runs, which differed in terms of the user's viewpoint of the task space. The first was an orthogonal view of the cursor (cannon) and the target (arrow) from directly above the pivot point of the cannon. The second was a perspective view of the cannon and arrow from a $45^{\circ}$ angle (above horizontal) at the same distance behind the cannon. The viewpoint was varied to determine the effect of perspective on frequency separated tracking performance. Within each of the five sets of experiments two tasks were evaluated: a docking task and a tracking task. The presentations of docking and tracking trials were combined and randomised in order to add an element of uncertainty to subject expectations. There were eighty-four docking trials and ten tracking trials per viewpoint presentation.

The experimental design is summarised in more detail below. This experiment consisted of a simultaneous combination of two factorial designs. The first design was a $6 \times 14 \times 2 \times 5$ factorial, within-subject design docking experiment. In particular, there were:

- six levels of displacement, or angular departure (AD), between the presented cursor and target markers (illustrated in Figure 21 ): $\pm 45^{\circ}, \pm 90^{\circ}$, and $\pm 135^{\circ}$, where the counter-clockwise rotation is represented by positive values, 
- fourteen levels of cursor orientation (CO), with respect to a nominal zero position, or base vector (illustrated in Figure 21): $\pm 0^{\circ}, \pm 45^{\circ}, \pm 90^{\circ}, \pm 120^{\circ}, \pm 135^{\circ}, \pm 160^{\circ}, \pm 180^{\circ}$ (the presentation pairs for cursor positions of $\pm 0^{\circ}$ and $\pm 180^{\circ \circ}$ were presented twice and counter-clockwise is the positive direction),

- two levels of viewpoint perspective (VP), that is:

- view vector top-down, or $0^{\circ}$ above, or plan view; and

- perspective view vector, or pitched down $45^{\circ}$, or view-from-behind,

- five levels of dynamic tethering (DT), in terms of natural frequencies: $w 00: 0 \mathrm{~Hz}$, w01: $2.646 \times 10^{-3} \mathrm{~Hz}$, w02: $2.292 \times 10^{-2} \mathrm{~Hz}$, w03: $1.985 \times 10^{-1} \mathrm{~Hz}$, and w99: $\infty \mathrm{Hz}$.

It is important to understand the distinctions between the three different independent variables presented above as angular displacements. The first, $\mathrm{AD}$, represents the size of the step input to the docking task. The second, $\mathrm{CO}$, represents the angular rotation of the cursor relative to the defined forward facing base vector, and is unrelated to $A D$, the size of the docking task. The third, VP, represents the angular rotation of the camera viewpoint about its pitch axis relative to the cannon control system.

The second design was a 10x2x5 factorial, within-subject design tracking experiment. In particular, there were:

- ten levels of tracks, reflecting the average cursor angular displacement (CAD) from the nominal zero position (as determined by the initial phase position of the track generator): $+1^{\circ},+22^{\circ},-40^{\circ}, 47^{\circ}, 102^{\circ},-114^{\circ},-121^{\circ},-131^{\circ},-165^{\circ}$, and $+165^{\circ}$.

- two levels of viewpoint perspective (VP, same as above), and

- five levels of dynamic tethering (DT, same as above). 
Once again, it is important to understand the distinctions between the two different angular independent variables presented above. The first, $\mathrm{CAD}$, is analogous to $\mathrm{CO}$ for the docking task, but represents a mean level rather than a single value. The second, VP, is identical to that of the docking conditions. There is no variable analogous to $A D$ above, since this variable is incorporated into the amplitude of the forcing function disturbance.

There were five different condition orders for any one trial block. The ten tracking tasks were randomly interspersed within the randomised docking task presentations. Since each subject was presented with each tether condition twice (once for each viewpoint, VP), they were exposed to each within-trial order twice. The order in which a trial block was presented to each subject was randomised. View vector and tether value were counterbalanced between subjects, however. Each subject was presented with a different dynamic tether each trial day, but with the same viewpoint order.

The within subjects design in this experiment was constructed not only to eliminate any biasing of the experimental results by individual differences, but also to allow eventual subjective evaluation and comparison of each display technique (i.e. each combination of tether parameters). In an attempt to minimise fatiguing and skill transfer (positive or negative) the following preventative measures were taken:

(1) Each set of tether parameters was tested on a different day for each subject, so that different tether conditions for any one subject were at least 16 hours apart. 
(2) Two-minute training sessions preceded each set of trials, to serve as a "buffer" to reduce transfer from the session of the previous day and to eliminate any surprise that may have arisen due to the novelty of the display dynamic.

(3) Lastly, the order of the five tether conditions tested were counterbalanced over the ten subjects, using a Latin square pattern, which resulted in every tether condition being presented an equal number of times as either first, second, third, fourth, or fifth. (More details about the design can be found in Appendix A).

\subsubsection{Participants}

Ten paid subjects ( 2 women, 8 men) of varying backgrounds participated voluntarily in this experiment. Nine of the subjects had a fairly technical background; only one subject did not. Of the ten participants: five were engineers / researchers, four were students (with engineering or computer-related experience), and one was a promotional representative (who was also the subject who lacked a technical background). The subjects had normal or corrected to normal vision, and ranged in age from their late teens to late thirties.

All subjects had extensive previous experience interacting with computers. Only two of the ten subjects had any tracking experience, but none had any practice with the misalignment conditions presented here. Three of the participants were left-hand dominant, but all participants indicated that they used their right hands when manipulating computer input devices, such as a joystick or a mouse. Two of the lefthanded subjects performed counterbalanced trial orders. 


\subsubsection{Procedure}

The subjects were instructed to sit in a chair and adjust its height and position such that they were approximately $50 \mathrm{~cm}$ from the computer monitor, with their arm resting comfortably on the controller. Only 1 degree of freedom of the hand controller was active, that controlling the yaw of the cursor. The subjects then read a scenario describing the context of the task (a copy of the scenario/instructions that were given to the subjects can be found in Appendix C - Subject Information Package) and were then allowed 4 minutes to practice tracking and docking, with the hand controlier set at an optimal gain for the current value of dynamic display. The subjects were each instructed to acquire the target as quickly as possible in the case of the docking task, and to follow the target as closely as possible whenever a dynamic tracking scenario was presented.

The two experimental runs lasted approximately 20 minutes each. Each run consisted of 84 docking trials and 10 thirty second tracking trials. The average completion time of a docking trial was 5 seconds, so the total session duration per subject was approximately 40 minutes per day, excluding individual differences. Each subject ran through the experiment 5 times (once a day for five days) for the five different tethering values..

While performing the tracking tasks subjects received feedback from a small window placed in the upper-left corner of the interface, indicating their completion time or RMS error. Following the completion of each trial, if the hand-controller was not re-centred, then the message window would indicate to the operator that this must be done before the 
next trial could begin. This measure belped to ensure that false recordings of reaction time and control reversals would be avoided.

Each subject was required to fill out a questionnaire both before beginning and after completing the entire experiment. The first questionnaire was used to collect demographic information on each of the subjects. Following execution of the experiment, each subject reviewed the experimental displays for all five sets of tethering parameters and performed subjective evaluations of the conditions. The subjective questions included the following:

(1) How intuitive was the display? (i.e. was the motion of the display understandable without explanation?),

(2) How do you feel the display condition affected your task performance? and

(3) Rate your response to the on-screen motion.

This subjective evaluation was important and was the main justification for adopting the within-subject design. We intended to compare these results with the actual performance data, so that a correspondence between the two could be made. 


\section{Experimental Results and Discussion}

In this study we are primarily interested in the effects of dynamic tethering on task performance. However, since we are also interested in how other factors interact with the tethering of a display, there are some main effects and interactions observed that are independent of tether mode (i.e., viewpoint perspective (VP), initial cursor orientation (CO), and cursor-target displacement (AD)). Although, some of these treatment effects by themselves are not of major concern in this study, all significant results are presented here in the sake of completeness, while only those of interest are discussed in detail in the following sections.

\subsection{Docking Task Results}

The docking task measures collected included (i) time-to-completion, (ii) percent overshoot, (iii) proportion of control reversals, and (iv) reaction time.

\subsubsection{Task Completion Time}

If dynamic tethering improves tracking performance then one might expect task completion times to decrease. After diagnostic checking of the statistical model data, transformations were applied to the data as necessary. Statistically significant results were detected for viewpoint (VP), and cursor-target displacement (AD) treatments. Interactions were found between tether type and viewpoint (DTxVP), and cursor orientation and cursor-target displacement (COxAD).

Data Transformations. All of the collected data for each measure were analysed using analysis of variance. When using this method of analysis certain assumptions must be 
made and the data must not provide evidence to the contrary if the results of the analysis tool are to provide reliable information to answer the posed research questions. Thus, diagnostic checking of the model was performed for every measure to ensure that the assumptions of the tool were adequately met.

One assumption of the analysis of variance statistical model is that the statistical residuals are independently and identically distributed according to a normal distribution, with a mean of zero and a fixed variance (Box, Hunter, and Hunter, 1978). The data for each measured variable was plotted to check if the plots roughly resemble samples of a normal distribution centred at zero. An analysis of the residuals computed from the docking measures indicated that all the collected data displayed these characteristics. A second assumption is that the behaviour of the residuals is independent of any one particular treatment. Again, an examination of the residuals gave no evidence of abnormal residual behaviour due to any of the treatments.

If the mathematical models are appropriate then the residuals should be unrelated to any of the known variables. This assumption holds for three of the four measures collected during the docking trials. However, the plot of the residuals of completion time versus the magnitudes of the completion time measures resulted in a funnel-like collection of data points (see Figure 24). This may indicate that the completion times had a constant percentage error. Such plots are not unusual when completion time is used as a performance measure. A log transformation is traditionally implemented in order to satisfy the residual distribution requirement for an analysis of variance (Howell, 1992 as 
cited by Zhai, 1995). The log transformation is a convenient transformation that results in a function with constant variance and thus the transformed data may be reliably analysed using analysis of variance (ANOVA). An analysis of variance was conducted on the log transformed task completion times; however, the results were then plotted on an untransformed scale for the sake of comprehension.

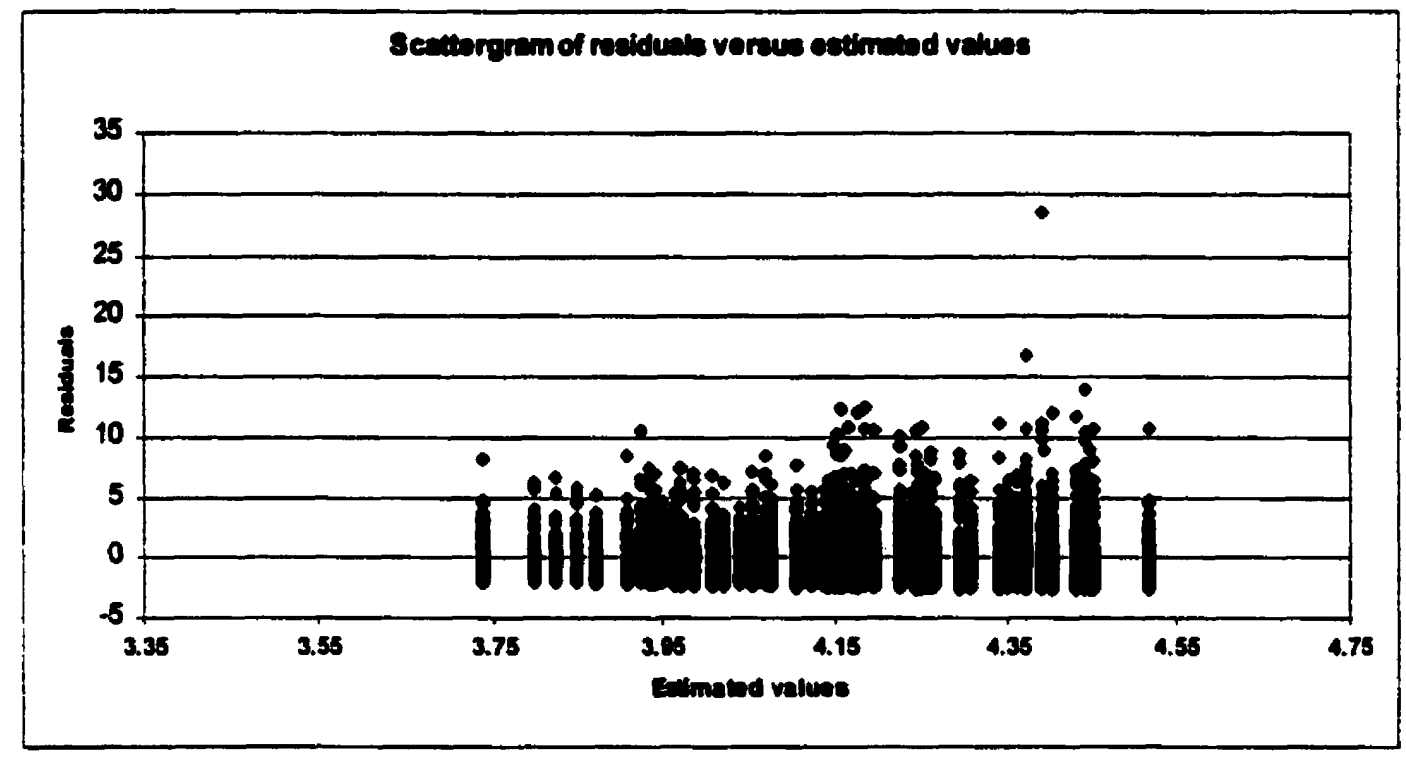

Figure 24 Analysis of Residuals: Plot of Residual Distribution for Completion Time

Task Completion Time - Viewpoint Perspective (VP) Main Effect:

The mean completion times calculated for the two viewpoints used in this study indicate that tasks performance with the perspective viewpoint took a significantly greater amount of time to complete than those performed with a top-down plan view $(F(1,9)=6.795$, $p<0.05)$. This is probably due to the fact that aligning the cannon with the arrow target in the virtual environment was aided by the presence of the straight edges of both objects in 
the planar (orthogonal) viewpoint. The difference between the two treatments is illustrated in Figure 25.

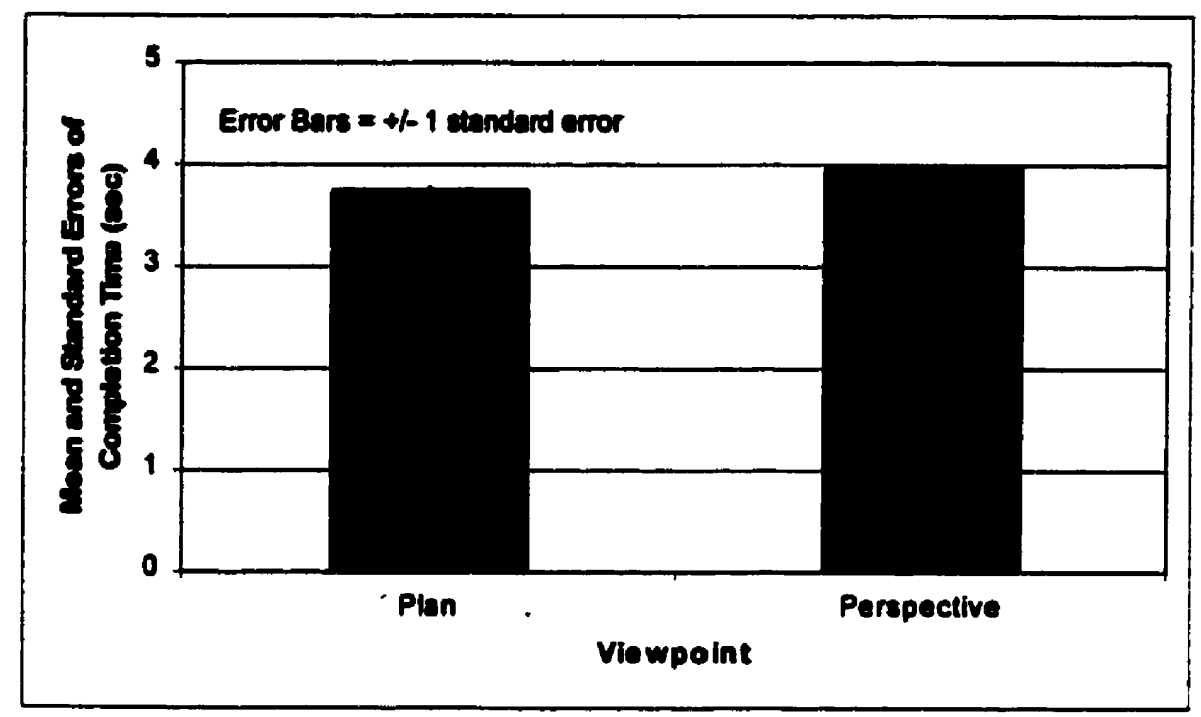

Figure 25 The effect of perspective (VP) on task completion time

Task Completion Time - Cursor-Target Displacement (AD) Main Effect:

Cursor-Target angular displacement $(\mathrm{AD})$ is the smallest ${ }^{17}$ angular distance between the initial target (i.e., arrow) position and the initial cursor (i.e., cannon) position. A strong significant main effect exists for cursor-target displacement angle, $F(18.04,1.94)=$ 83.535, $\mathrm{p}<0.0001$, but this is an expected result that is governed by Fitts' law, which predicts that, as the cursor-target displacement becomes larger, the time to complete a docking task increases. We do not plot this result for the sake of brevity, as it is neither a novel finding nor one of great interest to this study.

\footnotetext{
17 "smallest" refers to the direction and distance of travel to align the cursor and the target in these docking experiments
} 
Task Completion Time -- Tether-Viewpoint Interaction (DTxVP):

There is a significant interaction between the amount of dynamic tethering and the given viewpoint $(F(4,36)=2.982, p<0.05)$. This interaction is shown in Figure 26. This plot seems to suggest that using dynamic tethers with low values of natural frequency results in better tracking performance when given a planar view of the task space than when given a perspective view of that same task space. This result is presented here because of the statistical strength of the effect, however, it is not of large practical significance.

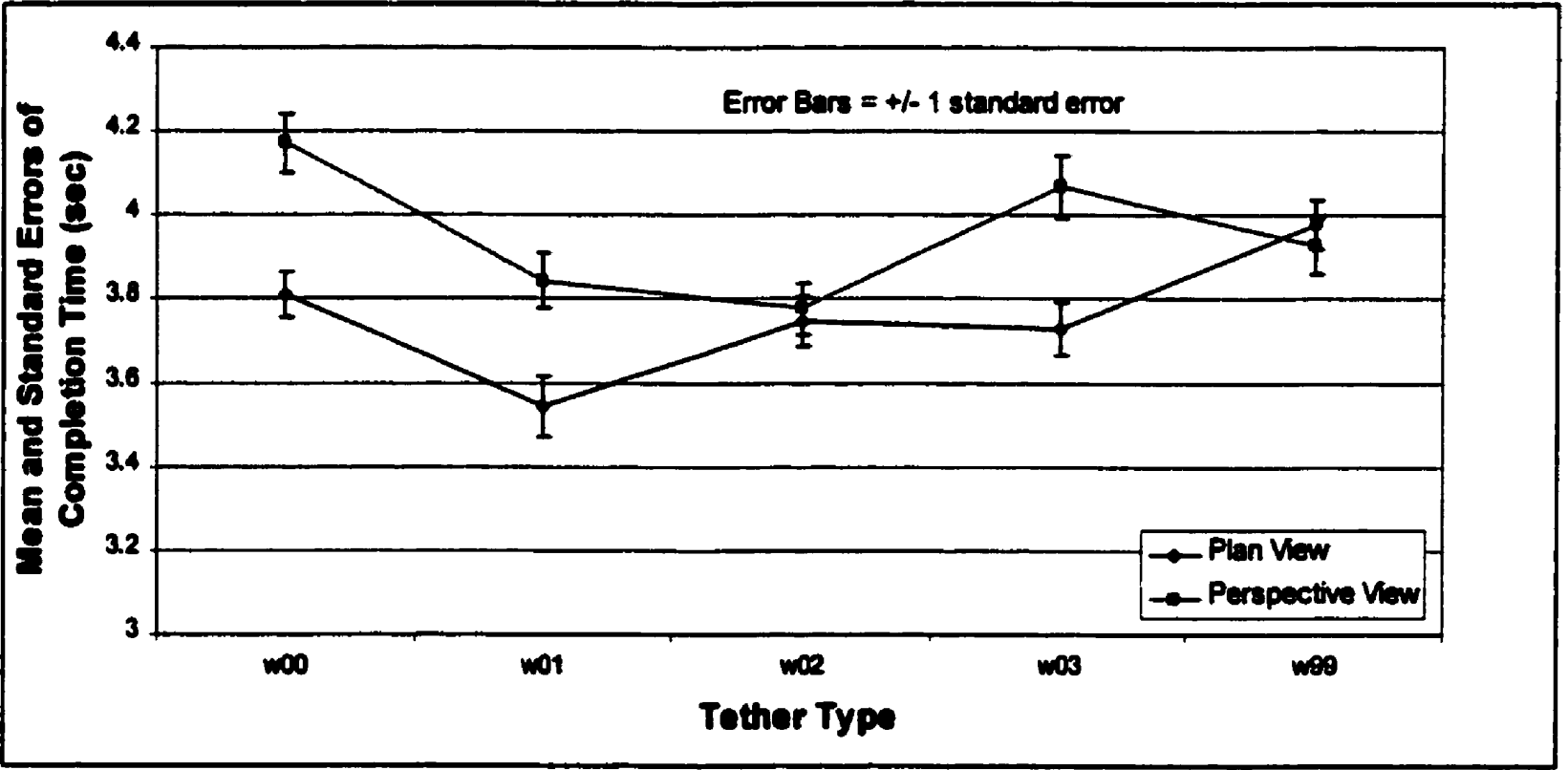

Figure 26 Task Completion Time: Tether $x$ Viewpoint Interaction (DT x VP)

Task Completion Time -- Cursor Orientation $x$ Cursor-Target Displacement Interaction (COXAD):

There is a marginal interaction ( $p=0.046)$ between cursor orientation and displacement across all tether types $(F(60,540)=1.354, p<0.05)$. However, this interaction does not 
appear to have any meaningful cause (see Figure 27 and Figure 28). The source of the interaction seems to be due to the symmetry of the cursor-target displacement treatment.

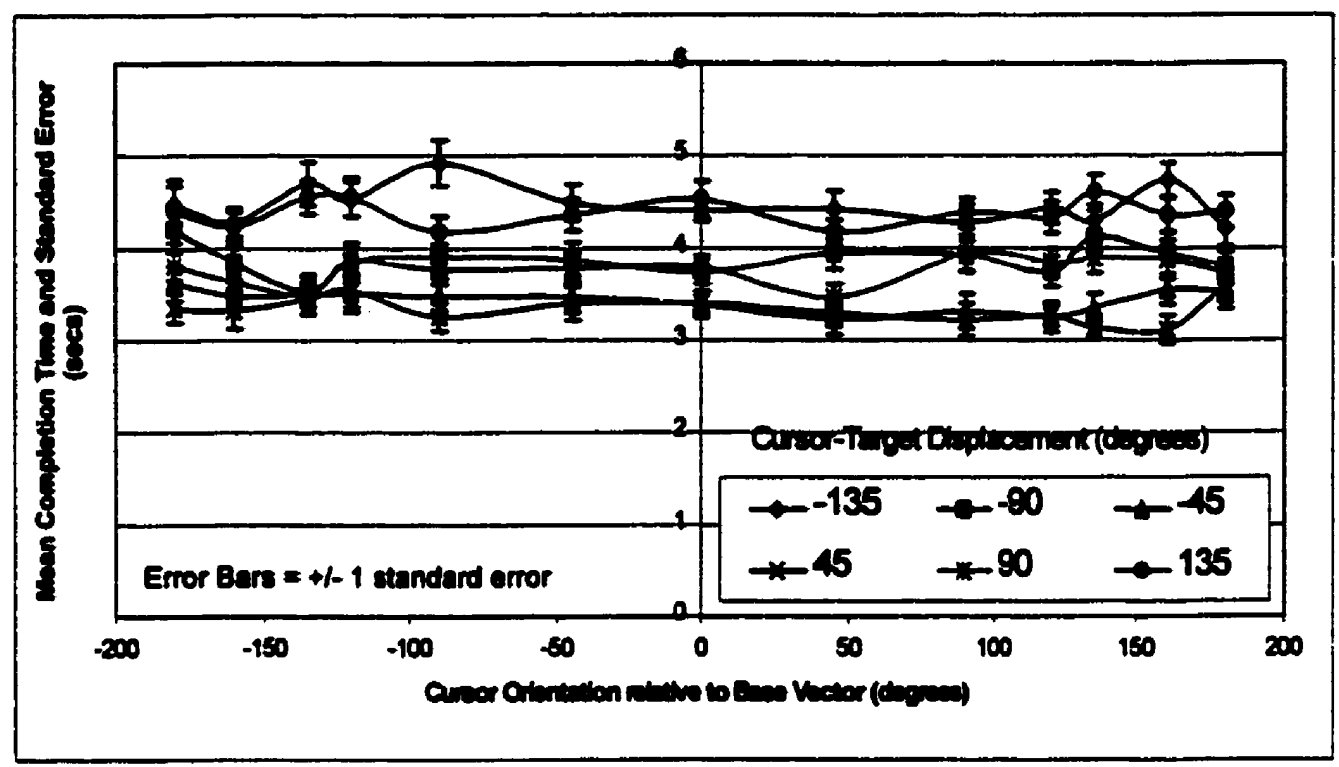

Figure 27 Task Completion Time: Cursor Orientation x Cursor-Target Displacement (CO $\times$ AD) Interaction (2 dimensional plot)
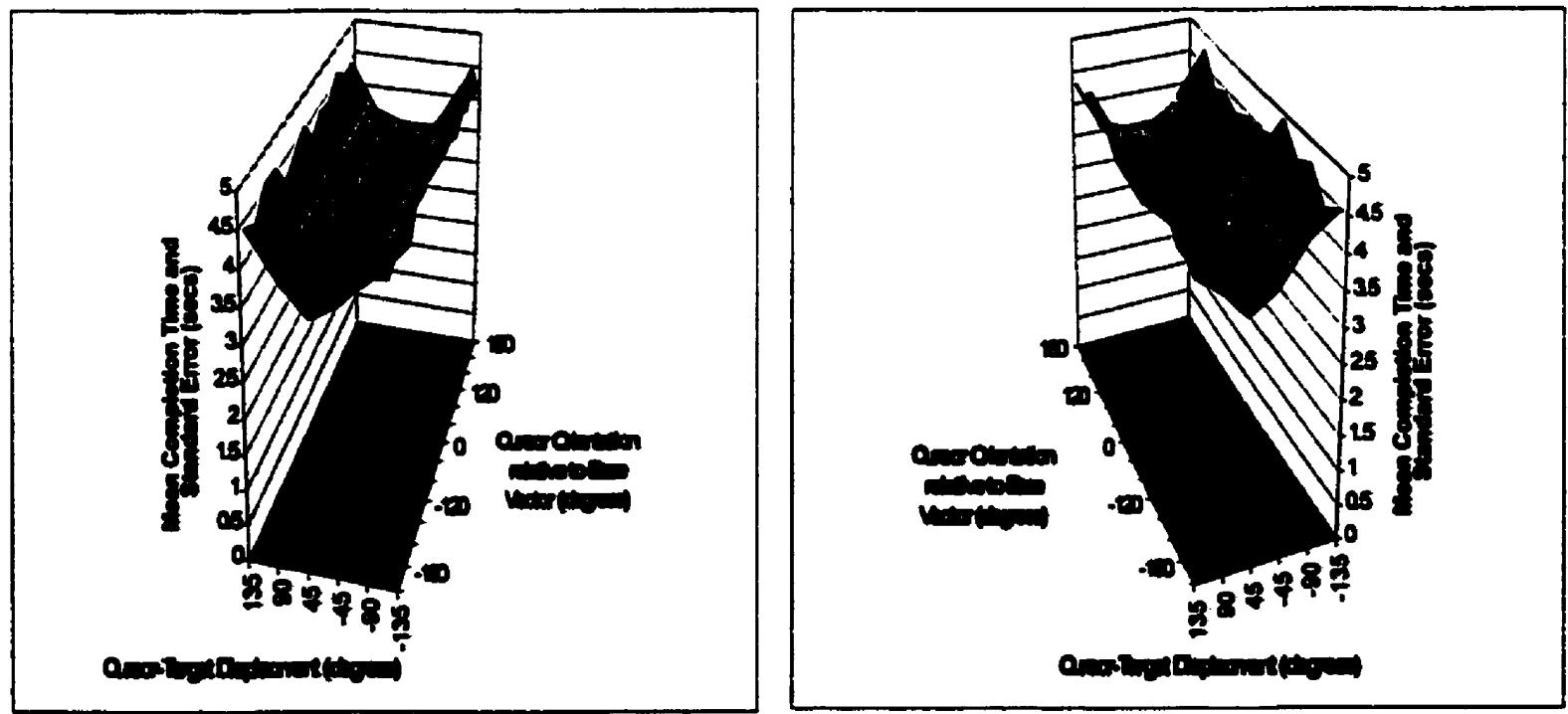

Figure 28 Task Completion Time: Cursor Orientation x Cursor-Target Displacement (CO $\times$ AD) Interaction (3 dimensional plots) 


\subsubsection{Percent Overshoot}

Target overshoot is a measure of control precision. Dynamic tethering appears to have no effect on the percent overshoot; however, cursor orientation and cursor-target displacement have produced statistically significant trends. Also, interactions were found between tether type and viewpoint, and between cursor orientation and cursor-target displacement.

\section{Percent Overshoot - Cursor Orientation (CO) Main Effect:} Cursor orientation ( $\mathrm{CO}$ ) refers to the direction and magnitude of the shortest angular displacement between the cursor (cannon) and the invisible base vector illustrated in Figure 21. Figure 29 illustrates percent overshoot as a somewhat U-shaped function of cursor orientation relative to the base vector (shown in Figure 21). Although a very significant main effect of cursor orientation was detected within the results $(F(12,108)=3.218, p<0.001)$ this result is not of great interest in this study. In addition, a Duncan multiple range test indicated that the only datum that is truly different from the rest is that at cursor displacement $+45^{\circ}$ from the (invisible) base vector $(\alpha=0.05)$. This Duncan test also revealed a few other groups of data that were reliably different from other groups of data plotted in Figure 29, but the groupings do not seem to result from any logical source of variance. This plot seems to indicate that when the cannon is initially aligned with the base vector (i.e. $0^{\circ}$ ) the amount of overshoot resulting from acquiring a target is minimised. It thus seems that any other initial orientation will cause the operator to overshoot the target location and that this error increases with increased deviations from the nominal forward facing position (i.e., $0^{\circ}$ ). 


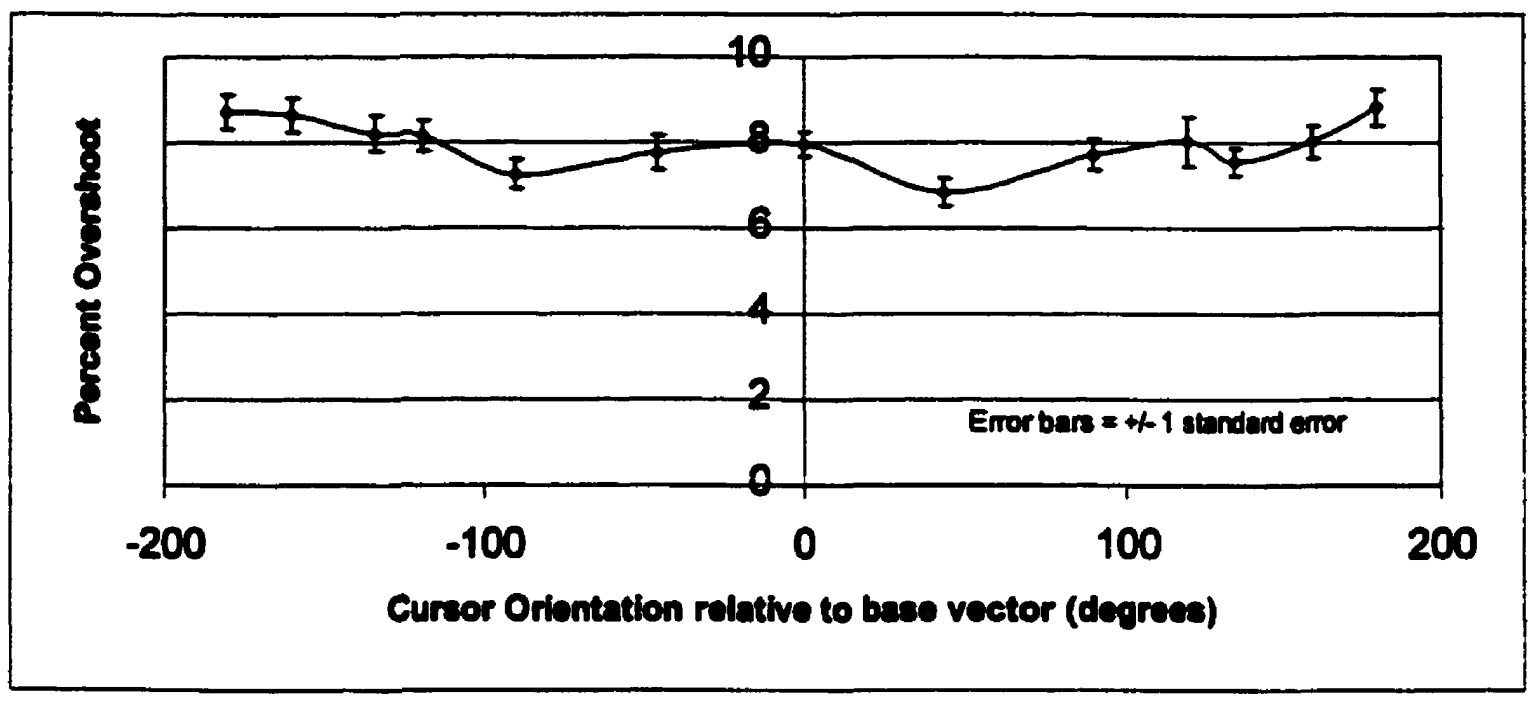

Figure 29 Effect of Cursor Orientation (CO) on Percent Overshoot

Percent Overshoot - Cursor-Target Displacement (AD) Main Effect:

The effect of cursor-target displacement (AD) as plotted in Figure 30 was an expected result. The overshoot caused by operator manual control at small cursor-target displacements is the result of the difficulty involved in precisely controlling the cursor with the given gain. Recall that the gains used for the docking tasks were optimised in terms of "average" completion time across all of the different displacement scenarios. Thus, a gain that is optimal for all displacements in general may not be optimal for the smallest or the largest of the cursor-to-target displacements. The result is that at small displacements the operator must react quickly to keep the cursor from overshooting the target. If she is not able to react quickly enough, then an overshoot occurs. Additionally, as the initial displacement approaches zero, the percent overshoot measure becomes inflated, as per (Equation 8-2). 
The shape of the above plot may therefore be the result of one or both of these factors. That is, either (1) the amount which the participant overshoots the target is relatively constant, regardless of cursor-to-target displacement magnitude and thus the curve is produced due to the 'inflation' effects of the overshoot equation and/or (2) the operator's control and precision increases as the larger displacements permit more time to acquire the stationary target - producing a curve that is a product of operator 'quickness'. The plot of the data is not linear, suggesting that the latter line of reasoning is the main cause of this response. This relationship between the cursor-target displacement (AD) and percent overshoot is strong $(F(5,45)=83.915, p<0.0001)$.

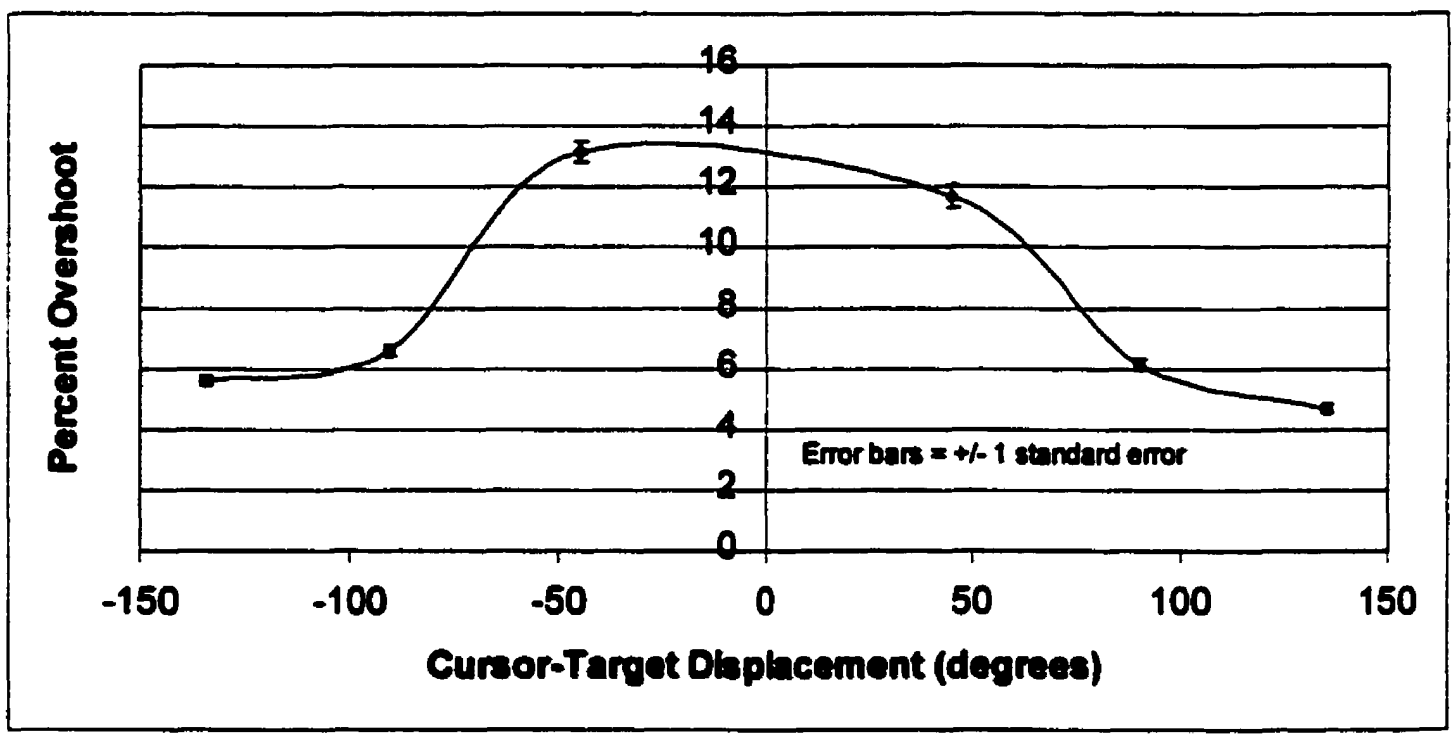

Figure 30 Effect of Cursor-Target Displacement (AD) on Percent Overshoot

\section{Percent Overshoot - Cursor Orientation $\times$ Cursor-Target Displacement (CO $\times A D)$ Interaction:}

At low cursor-target displacement $(\mathrm{AD})$ values (i.e., at $\pm 45^{\circ}$ ) the variances in percent overshoot measures with changes in cursor orientation are quite different than the more 
consistent measures obtained with higher displacements (i.e., $\pm 90^{\circ}$ and $\pm 135^{\circ}$ ). This result is plotted in Figure 31 . The changes at this $\pm 45^{\circ}$ cursor-target treatment level are very significant $(F(60,540)=2.301, p<0.0001)$. The increased variation at lower displacement values is a result of the apparently increased difficulty associated with coordinating small control movements while performing necessary mental rotations to accommodate for discrepancies caused by cursor orientation (CO). This increase in task difficulty results in a larger variance in the percent overshoot measures, as well as, an increase in percent overshoot that occurs when the magnitude of the cursor's orientation (CO) relative to the base vector increases. It is suspected that this effect might be more pronounced in the non-tethered $(w 00)$ treatment case, but there is no evidence of a tether $\mathrm{x}$ cursor orientation $\mathrm{x}$ cursor-target displacement interaction to support that theory $(F(240,2160)=1.084, p=0.192)$. 


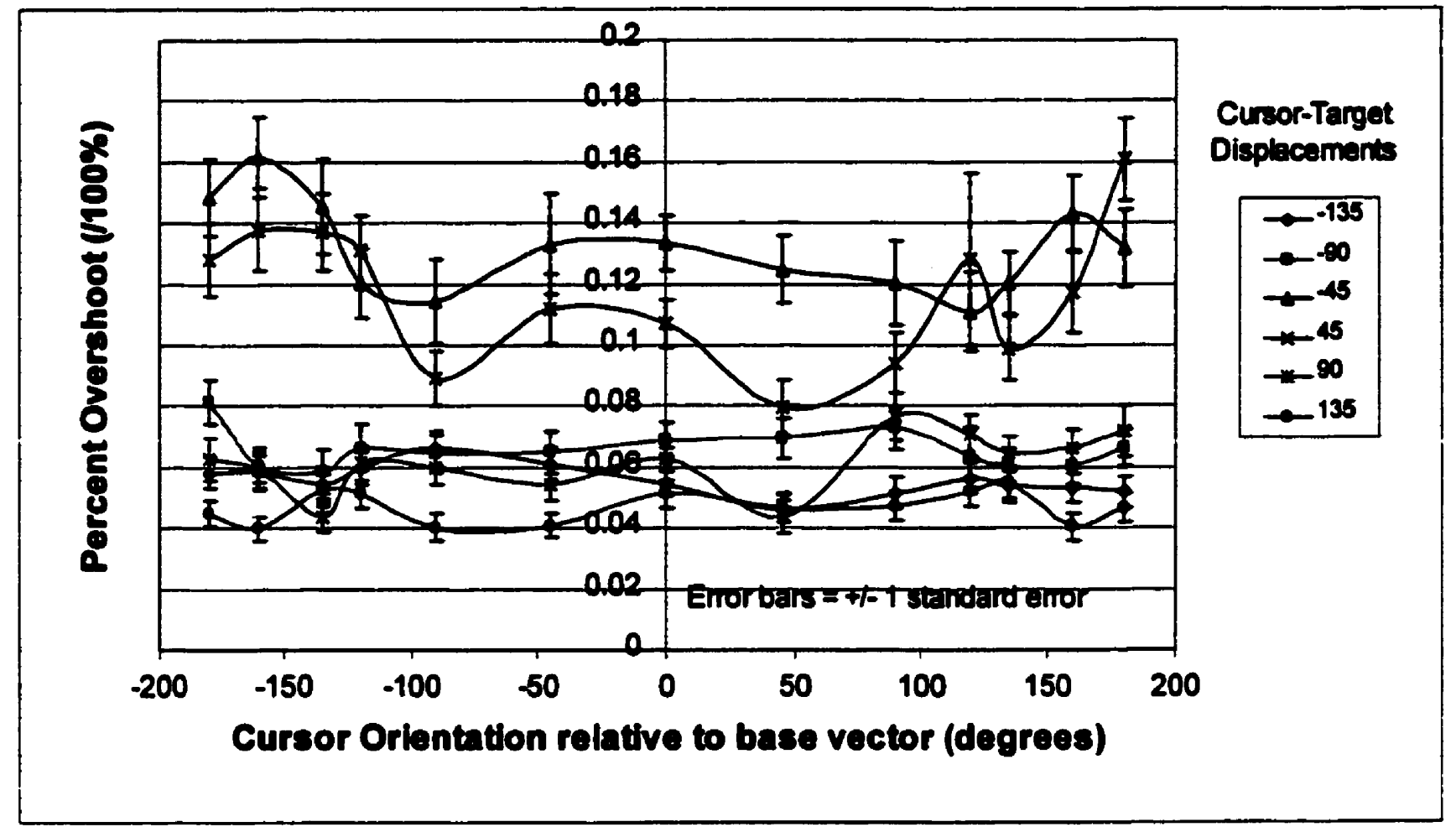

Figure 31 Cursor Orientation x Cursor-Target Displacement (CO x AD) Interaction

Percent Overshoot - Tether $x$ Viewpoint (DT $x$ VP) Interaction:

The plot below (Figure 32) does not reveal any important information about the interaction between the tether treatments and the viewpoint treatments that might impact this study in any meaningful way. However, there is a significant interaction between the two treatments $(F(4,36)=4.593, p<0.005)$. This may indicate that significant dymamic tethering improves performance in terms of percent overshoot given a planar view of the situation, but decreases percent overshoot when given a perspective view and a fairly weak or non-dynamic tether (i.e., w00, w01 and w99). Thus the dynamically tethered display seems to increase docking precision when given a plan view of the task space, relative to the more the traditional pursuit (non-tethered) and compensatory-like (rigidlytethered) displays. It is not clear why this weak $(p<0.005)$ relationship exists. 


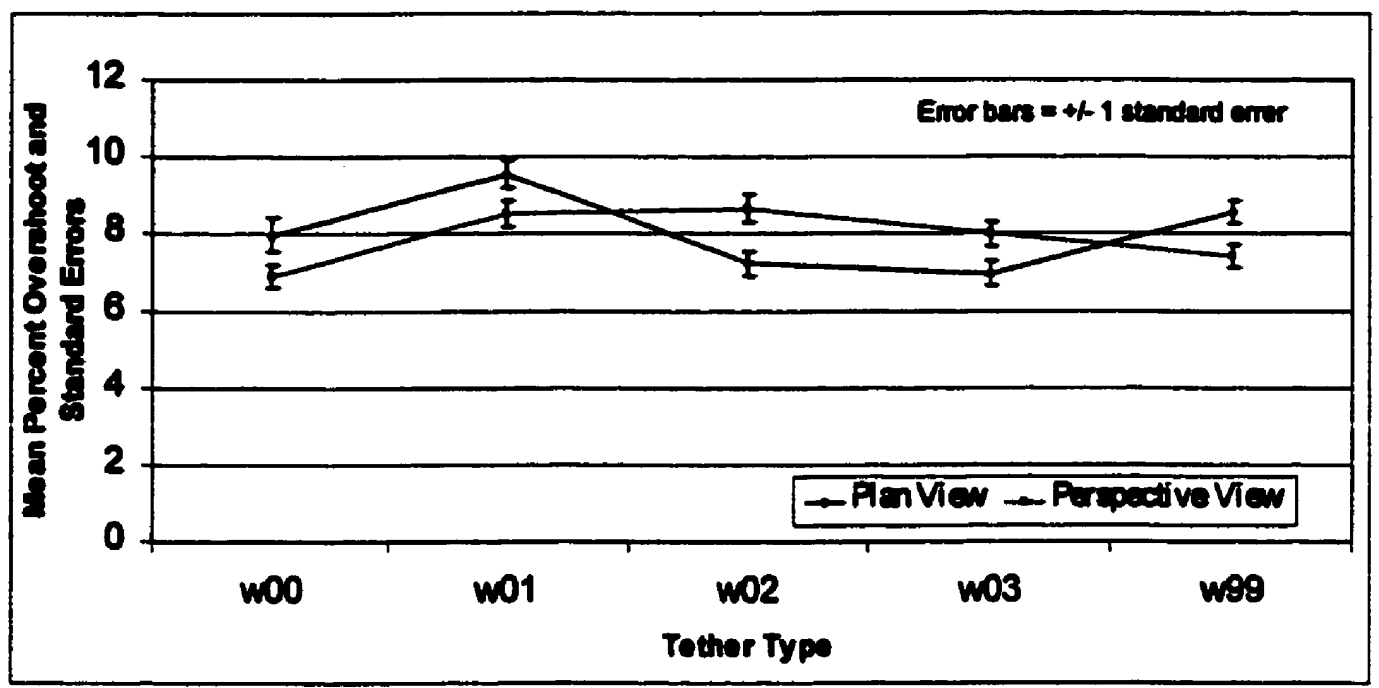

Figure 32 Percent Overshoot: Tether x Viewpoint (DT x VP) Interaction

\subsubsection{Reaction Time}

The reaction time varies as a function of tether type (DT), cursor orientation (CO) and cursor-target displacements (AD). These effects are discussed in the following paragraphs.

Reaction Time -- Dynamic Tether (DT) Main Effect:

Tethering (DT) significantly decreases the reaction times associated with the docking tasks of this experiment $(F(4,36)=18.343, p<0.0001)$. This result is illustrated in Figure 33. Results of Duncan multiple range test indicate that a significant difference exists only between the w00 tether (i.e. non-tether) and the rest of the tether values $(\alpha=0.05)$. Tethers w01, w02, w03, and w99 produce essentially equivalent reaction time results. 


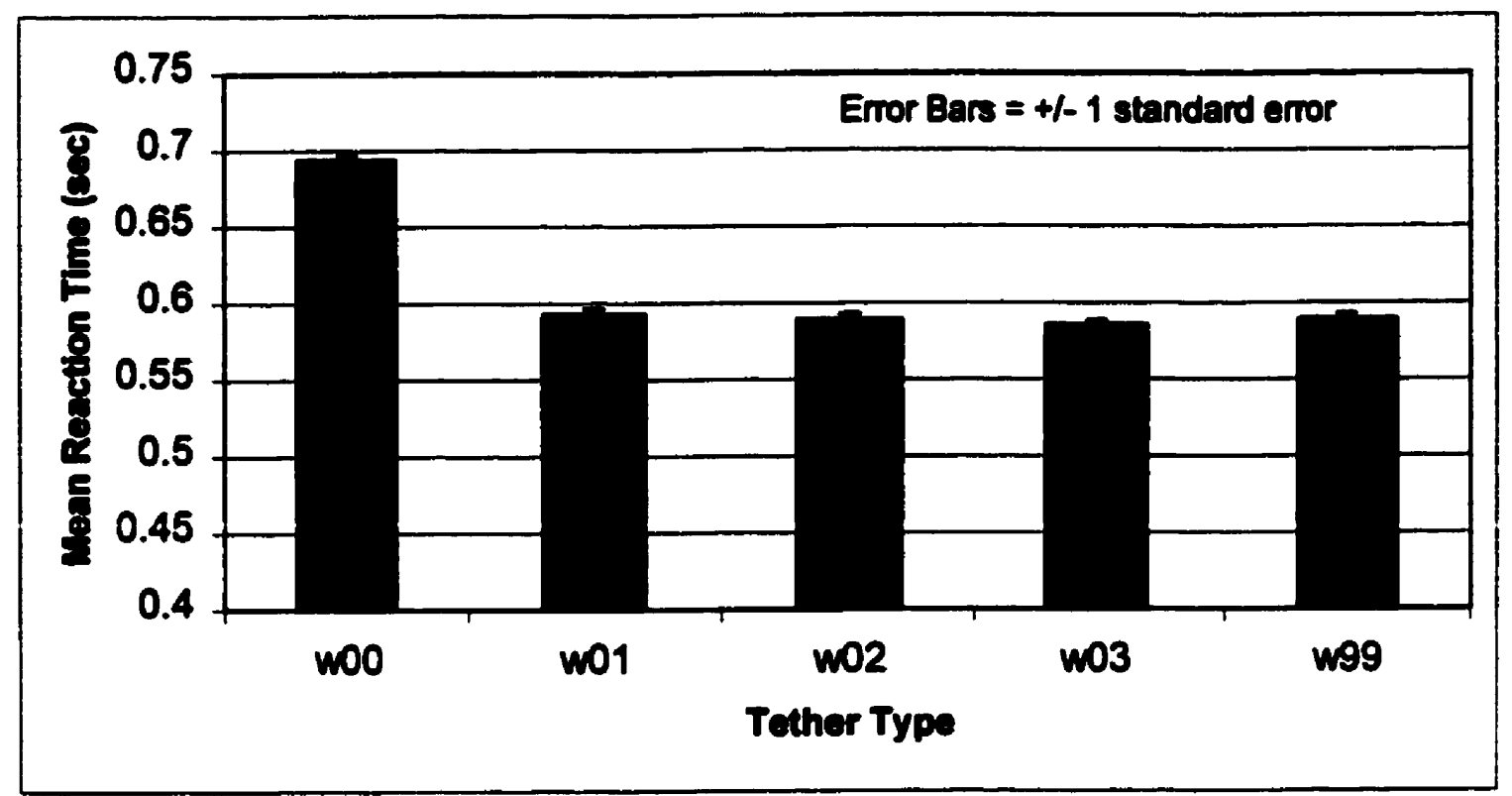

Figure 33 Effect of Dynamic Tethering (DT) on Reaction Time

In the non-tethered condition many of the trials contain varying degrees of controldisplay misalignment. The increase in reaction time associated with the non-tethered display is thus most likely due to the presence of these control-display incongruencies. It is these mismatches between the controls and the display which forced subjects to perform mental rotations, which in turn caused mental loading and thus increased processing time, which manifested itself as an increase in reaction time in the current task domain. Therefore, the advantage of tethered over non-tethered displays is apparent.

\section{Reaction Time - Cursor Orientation (CO) Main Effect:}

Figure 34 illustrates the effect of cursor orientation on reaction time $(F(12,108)=3.491$, $p<0.0001$ ). This effect seems to be the result of the influence of the non-tethered condition, although no significant tether-cursor orientation was detected $(F(48,432)$ 
$=1.075, p<0.346$ ) (see Figure 35). Here the speed-accuracy trade-off is apparent. Two curves are plotted in Figure 34; one is a plot of the original mean reaction time data over all the trials and the second is a similar plot of the reaction time averages re-calculated without the times associated with runs in which control reversals occurred. It is clear from the plot that when no control reversal errors are made the average time to action increases. Also, this plot indicates that, as cursor angular displacement increases so does the mean reaction time, suggesting that subjects may be comparing the presented image of the cannon to a mental image of the cannon in a canonical forward facing orientation (i.e. the turret in line with the base vector).

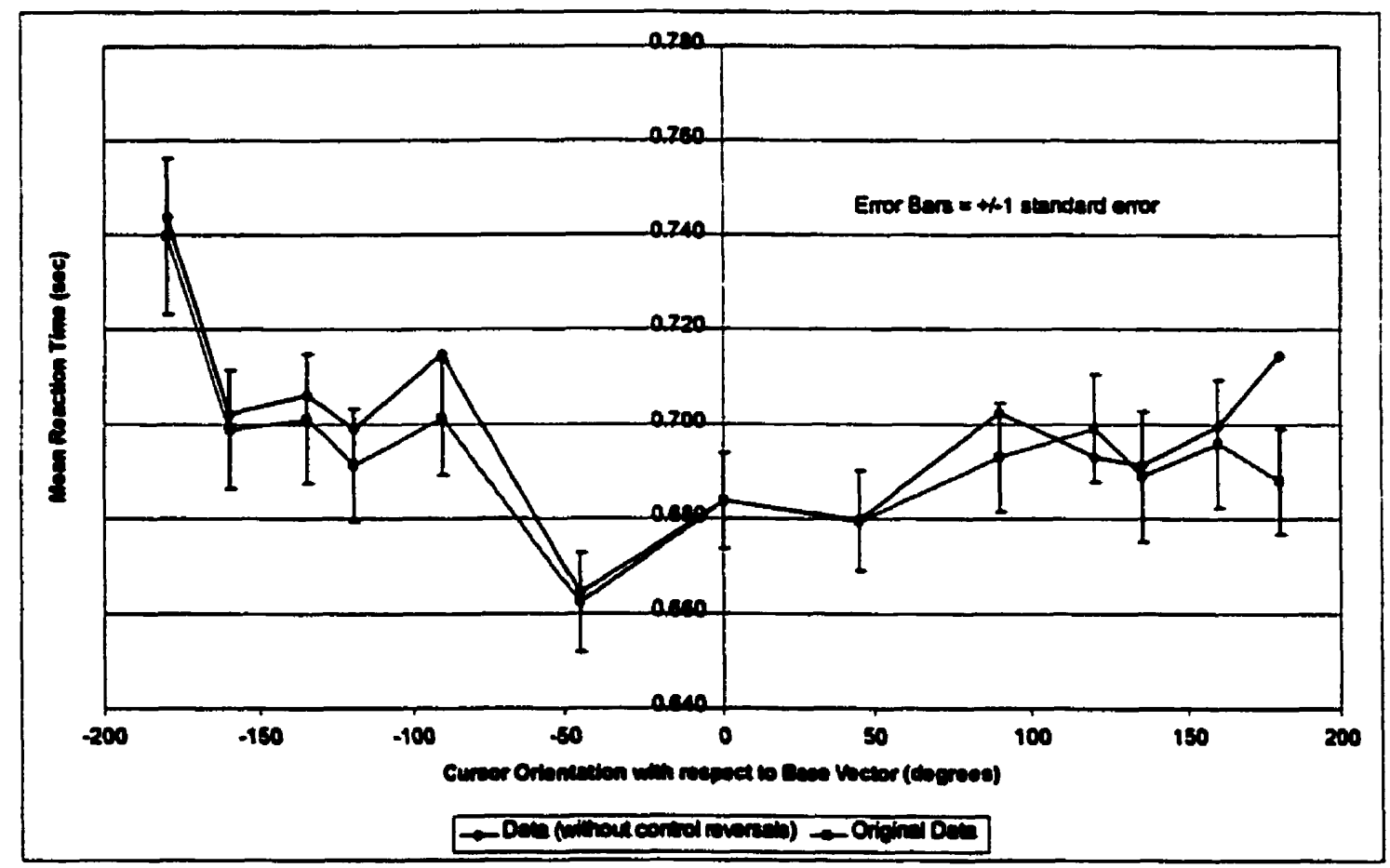

Figure 34 The effect of Cursor Orientation (CO) on Mean Reaction Time 


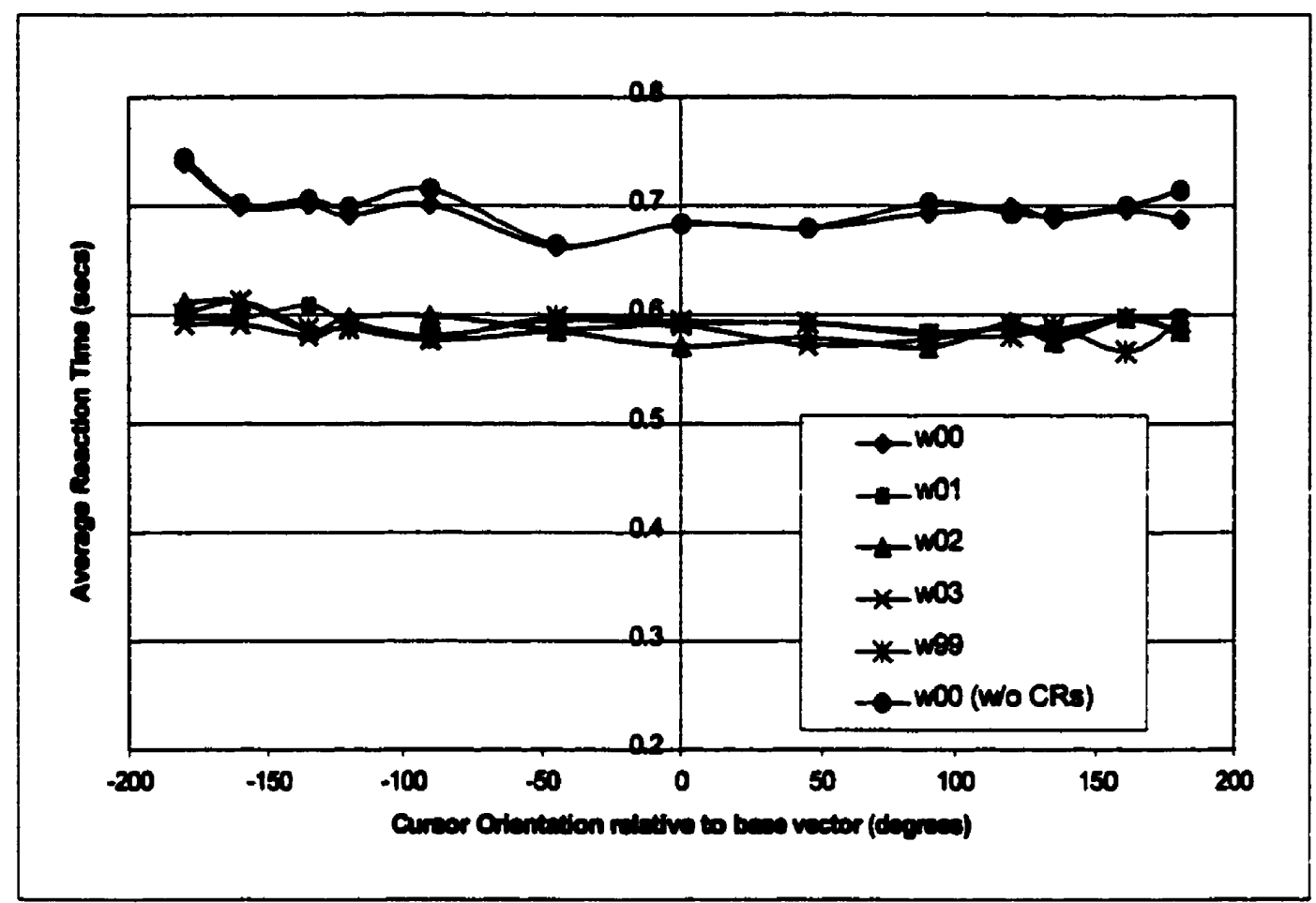

Figure 35 Plot of Mean Reaction Times recorded at each Cursor Orientation (CO) for each Tether Condition (DT)

A separate analysis of variance on the data for the w00 tether reaction time results alone show that there is a very marginal main effect of cursor orientation, i.e., $F(12,108)=1.666$ and $p=0.084$. The plots produced (in Figure 35 ) from the w00 data, in which control reversals did not occur ("w00 w/o CRs"), again illustrate that reaction time clearly increases with increasing cursor deviation from the orientation of the base vector (i.e. increasing control-display incongruency). Again here it can be seen that tethering eliminates the dependence of reaction time on cursor orientation.

There appears to be also an additional unexpected benefit of the tether illustrated above. It seems as though there is an additional decrease in mean reaction time associated with "anticipation". In the tethered cases the subjects anticipated the presence of immediate 
control-display congruence. This expectation allowed them to prepare to make a faster decision in determining the direction of input, maybe due to simplification of the decision process. Assuming that mental rotation operations before motor task execution are:

(1) determining if a mental rotation is required,

(2) mentally rotating the display into alignment with the controls, or vice versa, and,

(3) determining the direction of required input by mentally rotating the cursor to the target, or vice versa,

then we may attribute the 0.9 second gap in

Figure 35 between the non-tethered display curve and the tethered display curves at $0^{\circ}$ cursor orientation to a reduction in information processing requirements. That is, the subjects have no need to ask themselves if a mental rotation is required before initiating the next control movement. Thus, the reduction of decision complexity required when using a tethered display instead of a non-tethered display can be assumed to result in a decrease in the mean reaction time associated with the task.

Reaction Time --Cursor-Target Displacement (AD) Main Effect:

There is a significant effect of displacement on reaction time $(F(5,45)=9.339, p<0.0001)$, as shown in Figure 36. A Duncan multiple range test $(\alpha=0.05)$ reveals that the data at $135^{\circ},+45^{\circ}$, and $+135^{\circ}$ can not be distinguished from one another and thus can be grouped together, while the data recorded at $-90^{\circ},-45^{\circ}$, and $+90^{\circ}$ can also not be reliably separated from each other and thus form another group. 
A general observation made from Figure 36 is that on average clockwise reactions (negative cursor-target displacements) are faster than counter clockwise reactions (positive displacements). The tasks that required a counter-clockwise mental/physical rotation of the cursor in order to align it with the target induced larger reaction times than the tasks that required clockwise rotations. This result may be due to one of two things: either (1) subjects are able to determine direction of rotation - via mentally rotating the cursor into alignment with the target - faster in the clockwise (negative) direction than otherwise, or (2) the asymmetrical resistance of the joystick affected performance in the counter-clockwise direction more than in the clockwise direction. The latter is most likely the true reason because during the experiment it seemed as though the joystick was beginning to wear from continuous use. As a result moving the joystick in the counterclockwise direction was notably more difficult than in the clockwise direction. The ease of movement in the clockwise direction thus produced quicker movements and faster triggering of the microswitch used to detect joystick rotations. This may also explain the unusual increase in reaction time at $+45^{\circ}$ cursor-target displacement. Subjects were more likely to take their time in moving the joystick in the counter-clockwise direction as the force they applied gradually overcame the "stiction" (static friction) while taking precaution not to overshoot the closely located target.

More importantly, if we observe the left and right sides of the plot separately, we find that reaction time generally increases with cursor-target displacement (provided we ignore the anomalous datum at $+45^{\circ}$ ). This finding seems to support step (3) in the hypothesised mental rotation process of operations described above. It would follow then 
that not only is a mental rotation required to attain control-display congruence but an additional rotation is required to imagine the rotation of the cursor into the target's position, thus causing an increase in the magnitude of the imagined rotation results and an increase in reaction time.

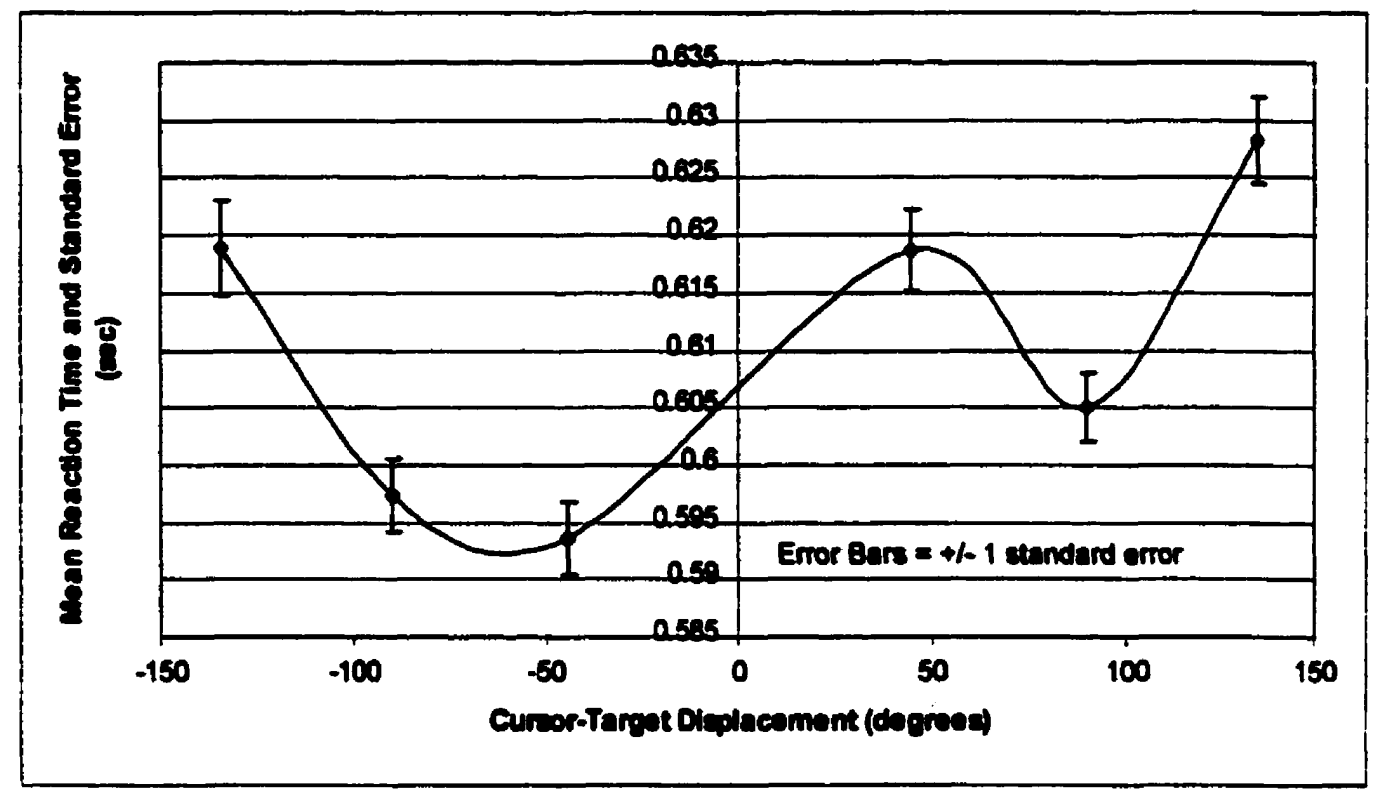

Figure 36 Effect of Cursor-Target Displacement (AD) on Mean Reaction Time

\subsubsection{Control Reversals}

The frequency of control reversals changes significantly with tether type and cursor orientation. A tether $\mathrm{x}$ cursor orientation (DT $\mathrm{x} \mathrm{CO}$ ) interaction and a tether $\mathrm{x}$ cursor orientation $\times$ cursor-target displacement (DT x CO x AD) interaction were also detected when analysis of variance was performed. These main effects and interactions are discussed in greater detail in the following paragraphs. 
Figure 37 indicates that using the non-tethered interface results in a reliably greater $(F(4,36)=12.889, p<0.0001)$ frequency of control reversals than observed when a tethered display is used. A Duncan multiple range test indicates that the result for tether w00 is the only significantly different measure $(\alpha=0.05)$. The results obtained with tethers w01, w02, w03, and w99 cannot be distinguished from each other. Thus, it is safe to conclude that they do not differ in terms of the number of control reversals likely to occur with each.

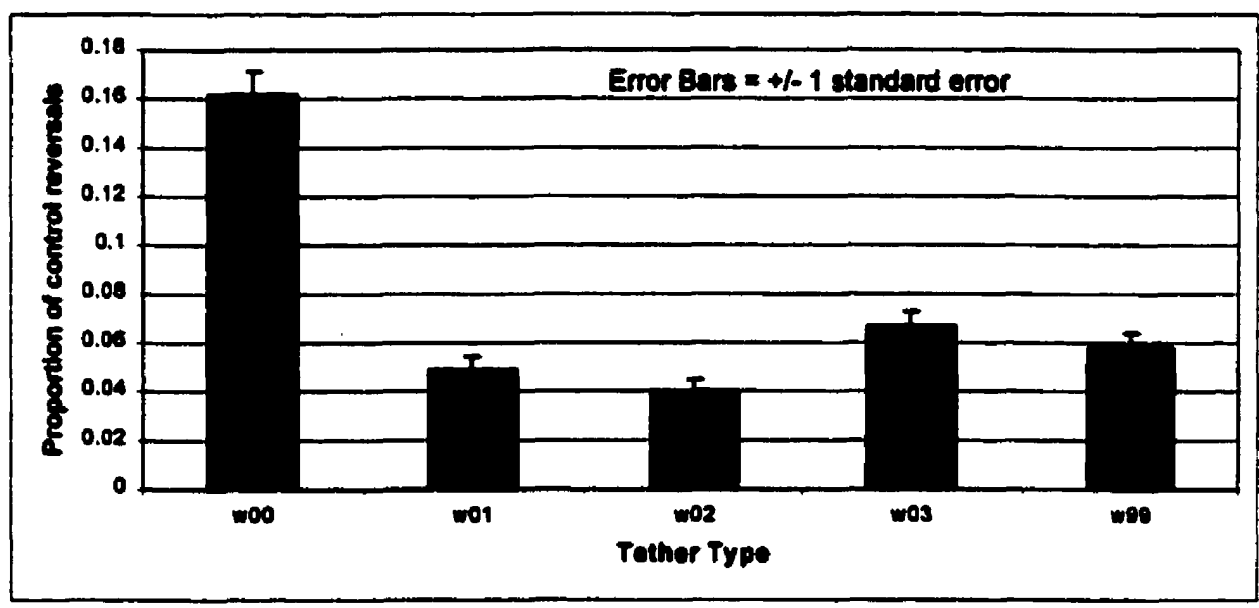

Figure 37 The effect of Dynamic Tethering (DT) on the occurrence of control reversals

The presence of a dynamic tether ensures that there is, at least, a high degree of controldisplay congruency when a docking task is first presented to the subject. Conversely, when control-display incongruencies are present, as in the non-tethered condition, these are likely to cause an increased frequency of control reversals. The reason for this is as follows. When a misalignment is present, the operator, knowing that he must act quickly, 
attempts to input his response to the system as quickly as possible, but sometimes fails to sufficiently complete mental rotation of the display enough to make an accurate decision that supports his choice of input. Such an apparent increase in frequency of incorrect decision making is illustrated well in Figure 37.

Control Reversals - Effect of Cursor Orientation (CO) on the Proportion of Control Reversals

\begin{abstract}
Although there seems to be a significant effect of cursor orientation (CO) on the occurrence of control reversals $(F(12,108)=2.516, p<0.01)$, this result is apparently due to a very strong interaction that occurs with tethering (presented in the next paragraph). Execution of a Duncan multiple range test $(\alpha=0.05)$ indicates that the values at the extreme ends of Figure 38 are significantly different from most (in particular 8) of the other data. As well the low frequency of control reversals that occur at $+45^{\circ}$ is significantly different from 3 of the highest proportion data.
\end{abstract}




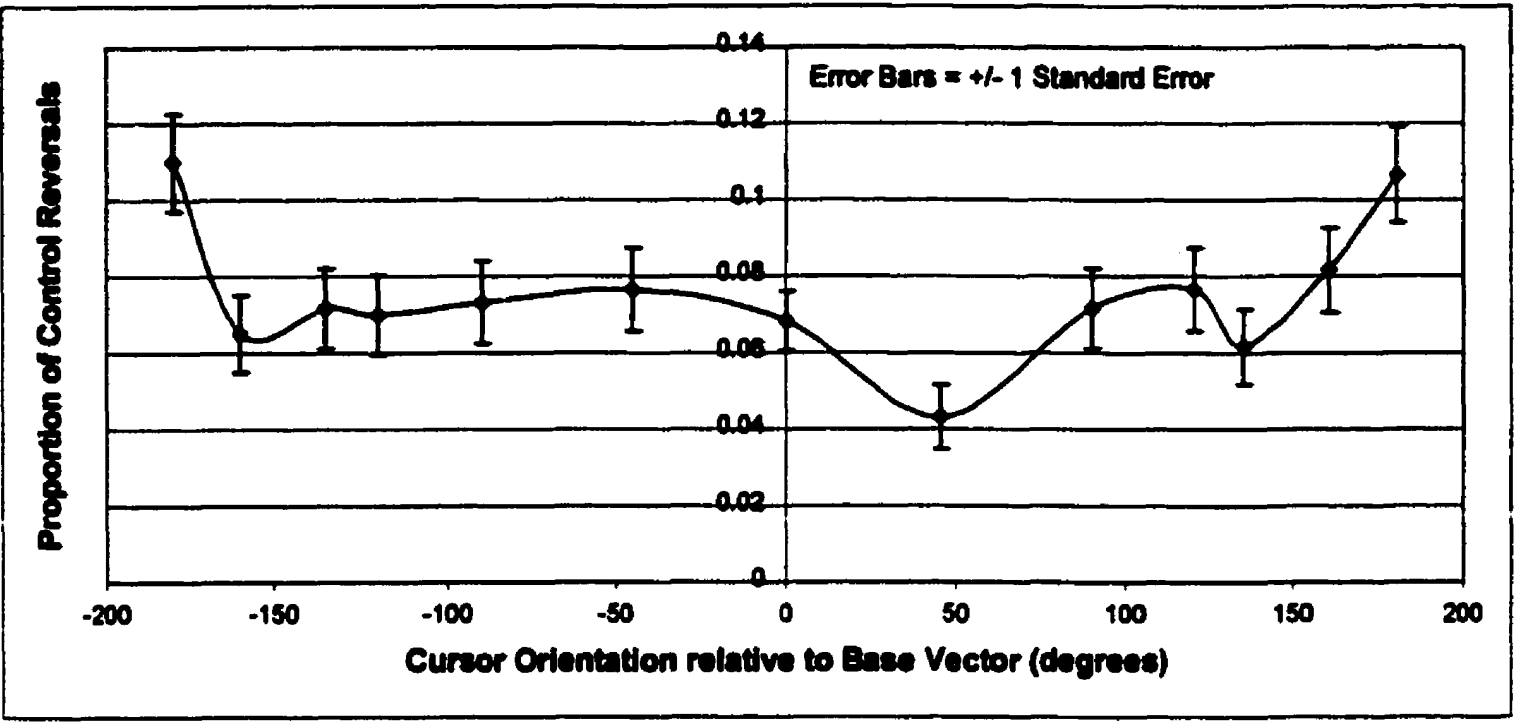

Figure 38 Proportion of Control Reversals as a function of Cursor Orientation (CO)

Control Reversals - Tether $\times$ Cursor Orientation (DT $\times C O)$ Interaction

When a viewpoint is tethered to the cursor (cannon) at the start of each docking trial, it is placed in a position equivalent to that of an assembly that has had time to reach equilibrium (i.e., there is no relative camera-cannon motion). The experiment was thus set up such that the controls and display are always aligned at the beginning of each docking trial. Tether treatment condition w00 reflects this congruency between the cursor and the controls only when the cursor orientation relative to the base vector is approximately zero. In this aligned condition the frequency of control reversals that occur is always low. A similar low frequency of control reversals is observed, however, for all values of cursor orientation for the tethered conditions, in addition to the values surrounding zero for the non-tethered condition, w00. 
When initial cursor orientation deviates significantly from zero for the w00 tether condition, this level of performance is not preserved $(F(48,432)=2.908, p<0.0001)$. The proportion of control reversals increase significantly at $\pm 90^{\circ}$ control-display misalignment, as was expected, due to the increased mental rotation operations that are involved in such a scenario. The radar plot in Figure 39 illustrates the symmetry in the results of clockwise and counter-clockwise input. It seems that there is some correlation between the magnitude of mental rotation required and the frequency of control reversals, that is similar to the relationship between metal rotation and time delay as previously discussed in section 9.2 .
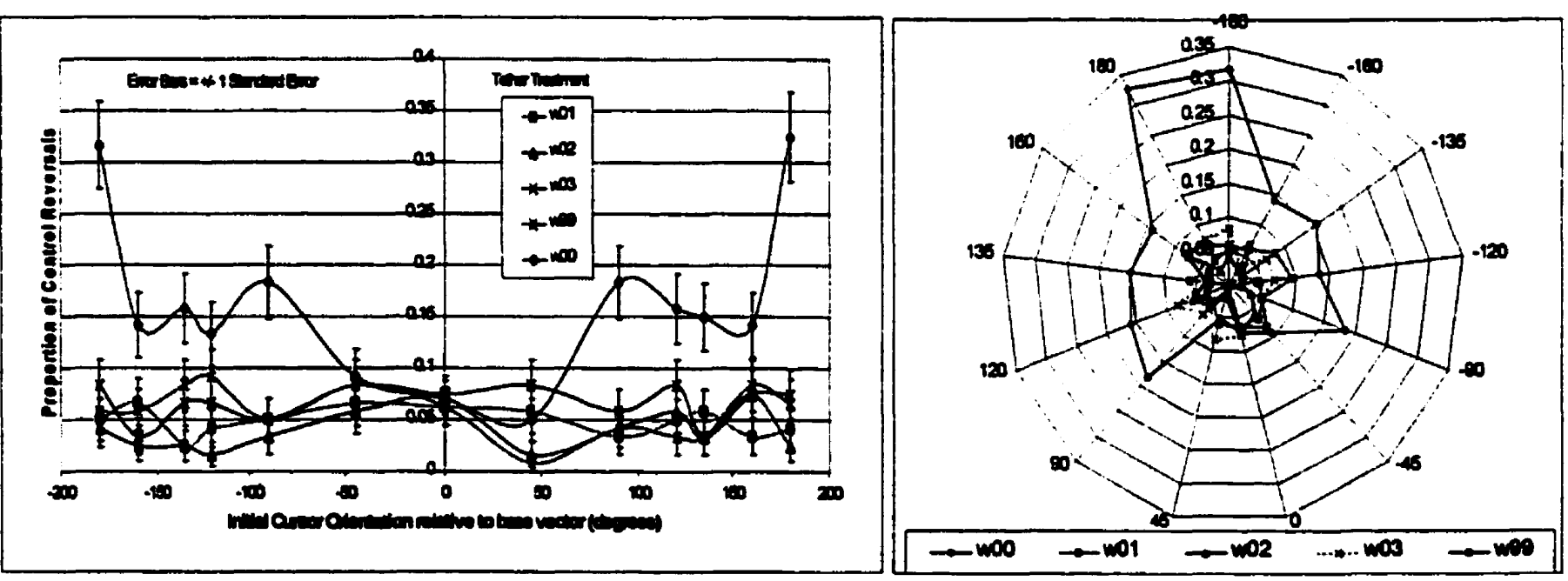

Figure 39 Control Reversals: Tether $x$ Cursor Orientation (DT x CO) Interaction These results are thus similar to those discussed by Huggins and Getty (1982, c.f. section 3.2.3). It would seem that subjects use a spatial strategy by directly mapping the displayed object (the cannon) to the controlled object (the joystick) at angular displacements close to $0^{\circ}$. A switch to a rotational strategy is apparent between $\pm 45^{\circ}$ and $\pm 90^{\circ}$. This accounts for the observed increase in control reversals with cursor orientation 
within the given range. It then seems that the relational strategy is implemented beyond $\pm 90^{\circ}$ resulting in no further increase in control reversals (i.e. the plot plateaux).

Alternatively, this plateau could be interpreted, as mentioned in Section 3.3.5, in the following manner. There may exist an angle (possibly $\pm 90^{\circ}$ in this case) beyond which the delay caused by a mental control reversal is acceptable, because the alternative rotation (e.g. to $180^{\circ}$ ) becomes so small that the combined mental rotation and reversal time is faster and possibly more accurate than performing a rotation into the compatible orientation. This alternative may also be used to explain why the plateau that occurs between $\pm 90^{\circ}$ and $\pm 180^{\circ}$ slants downward, i.e. the proportion of control reversals decreases as the required mental rotation, applied before the reversal strategy is applied, decreases. This second theory is supported by the data, in that the frequency of control reversals is directly proportional to the magnitude of the required mental rotation (and the time it involves).

However, something unexpected occurs at $\pm 180^{\circ}$ that is not supported by this second theory - the frequency of control reversals increases suddenly. This result also seems to indicate that neither the spatial nor relational strategies can be applied at these cursor orientations, possibly due to the symmetry of the cannon. This would correspond with subject reports that when they viewed the cannon in an unexpected "head-on" orientation they needed to consciously determine first which end was the front of the cannon. This additional operation may have involved a mental rotation to compare the image with a corresponding mental image of the canonical upward/forward facing cannon. Thus the subject is forced to rely on the slower and less accurate rotational strategy. 


\section{Control Reversals - Tether $\times$ Cursor Orientation $x$ Cursor-Target Displacement $(D T \times C O \times A D)$ Interaction}

There is a significant three-way interaction among tether treatment, DT, cursor orientation, CO, and cursor-target displacement, $A D(F(240,2160)=1.233, p<0.05)$. Figure 41 indicates, for tether w01, that there is no two-way cursor orientation $x$ cursortarget displacement $(\mathrm{CO} \times \mathrm{AD})$ interaction present when the display is tethered (the full set of five plots are included in Appendix B). However, the plot for the non-tethered display w00 (Figure 40) reveals the presence of such a two-way interaction, thus creating a three-way interaction.

The number of control reversals that occur at a displacement of $+135^{\circ}$ is unusually higher than that of all other displacement values. One might expect this result at least to be equivalent to that obtained with a displacement of $-135^{\circ}$. But this is not the case. We attempt to explain this result by referring to the experimental set-up. One particular aspect separates the results caused by large displacements from those created by smaller ones: the increased potential for incorrectly choosing the direction of smallest rotation. As cursor-target displacement becomes increasingly larger it becomes increasingly more difficult and/or requires increasingly more time consuming to determine the smallest angle between the cursor and the target (i.e., the correct direction of control rotation). This is further complicated in the non-tethered condition, in that there may also be a control-display misalignment that may make the task even more difficult. 
This possibly explains why there is a high frequency of control reversals at $\pm 135^{\circ}$, but alone it still does not explain the discrepancy between the $+135^{\circ}$ displacement condition and its negative counterpart. Again we may look to the joystick resistance and sensitivity for answers. Just as joystick wear has caused more resistance in the counter-clockwise direction and thus, apparently caused an increase in reaction times recorded in that direction, it may similarly reduce the number of control reversals committed in that direction when a movement in the opposite direction is the correct input. Thus some of the control reversals that might have been recorded for the $-135^{\circ}$ displacement condition are missed or, complementarily, the ease of movement in the clockwise direction allowed a greater number of control reversals to be recorded for the $+135^{\circ}$ displacement condition. This, coupled with the ease of initial misinterpretation of rotational direction for large displacement angles and the presence of control display incongruencies, explains the finding that, when $+135^{\circ}$ rotations are required, a greater number of control reversals were recorded than any other condition. This combination occurs only in the non-tethered case and thus a three-way interaction between tether, cursor orientation, and cursor-target displacement results. 


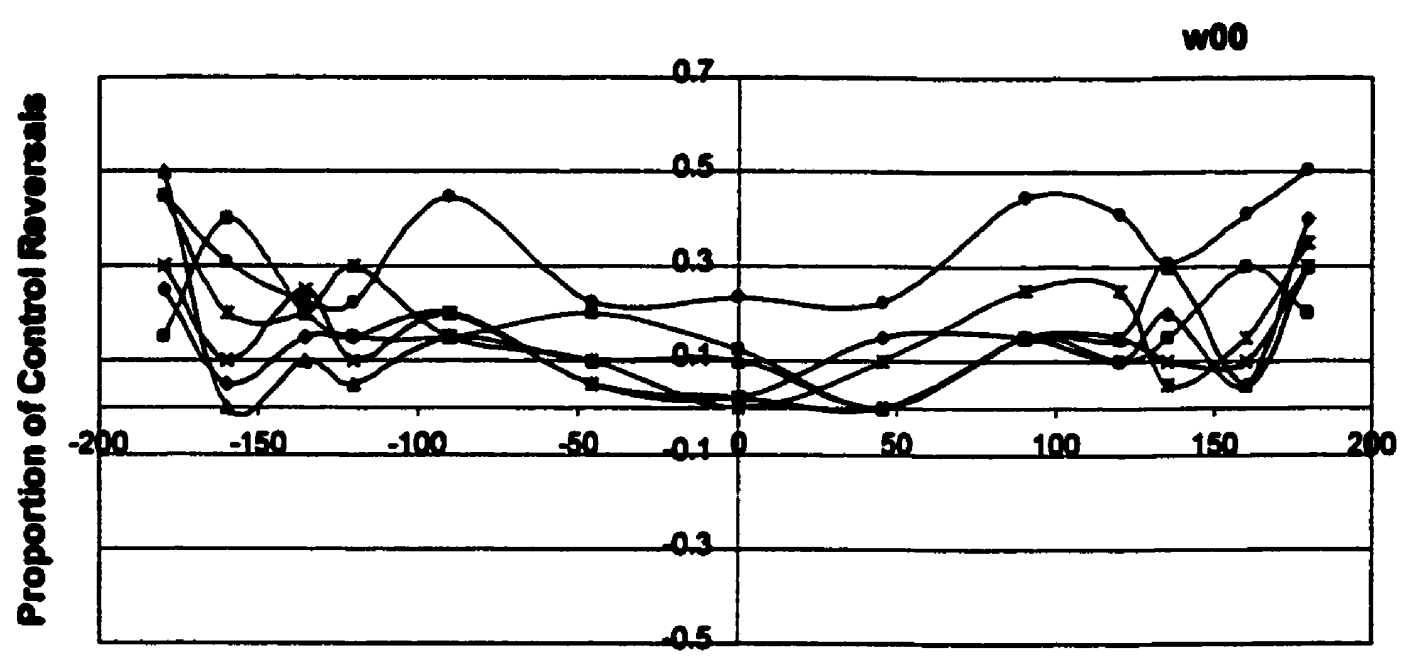

Curser Oribntation rolative to base vector (degrees)

Cursor-Target Displacoment (degreas)

$\rightarrow-135 \rightarrow-80 \rightarrow-45 \rightarrow-45 \multimap 90 \rightarrow 135$

(a)

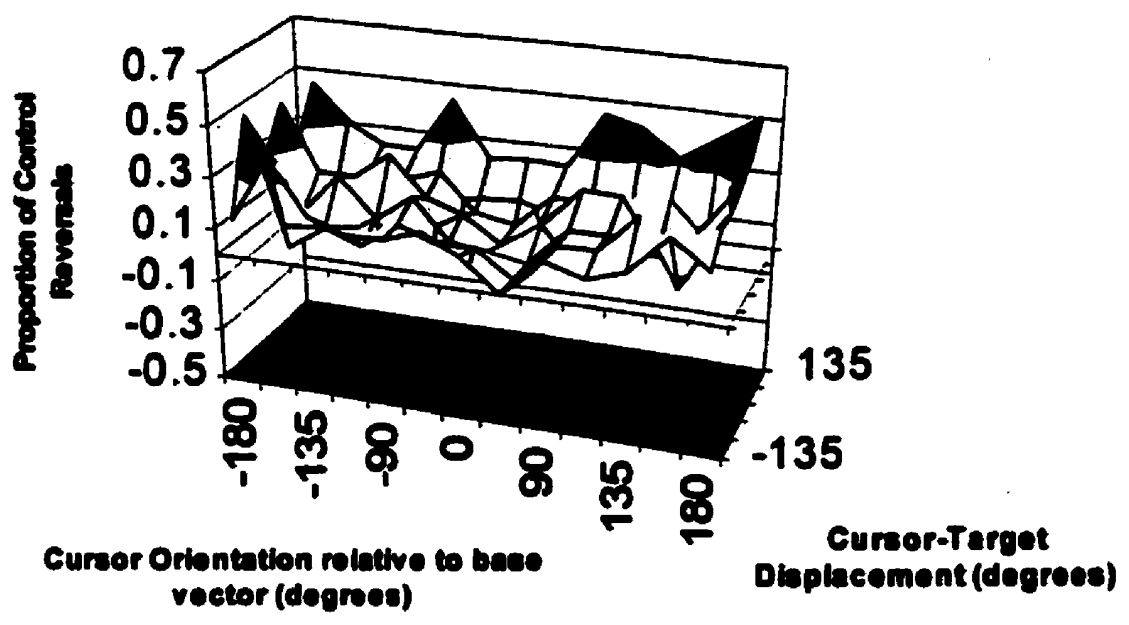

(b)

Figure 40 Cursor Orientation x Cursor-Target Displacement (CO x AD) Interaction for Tether w00 


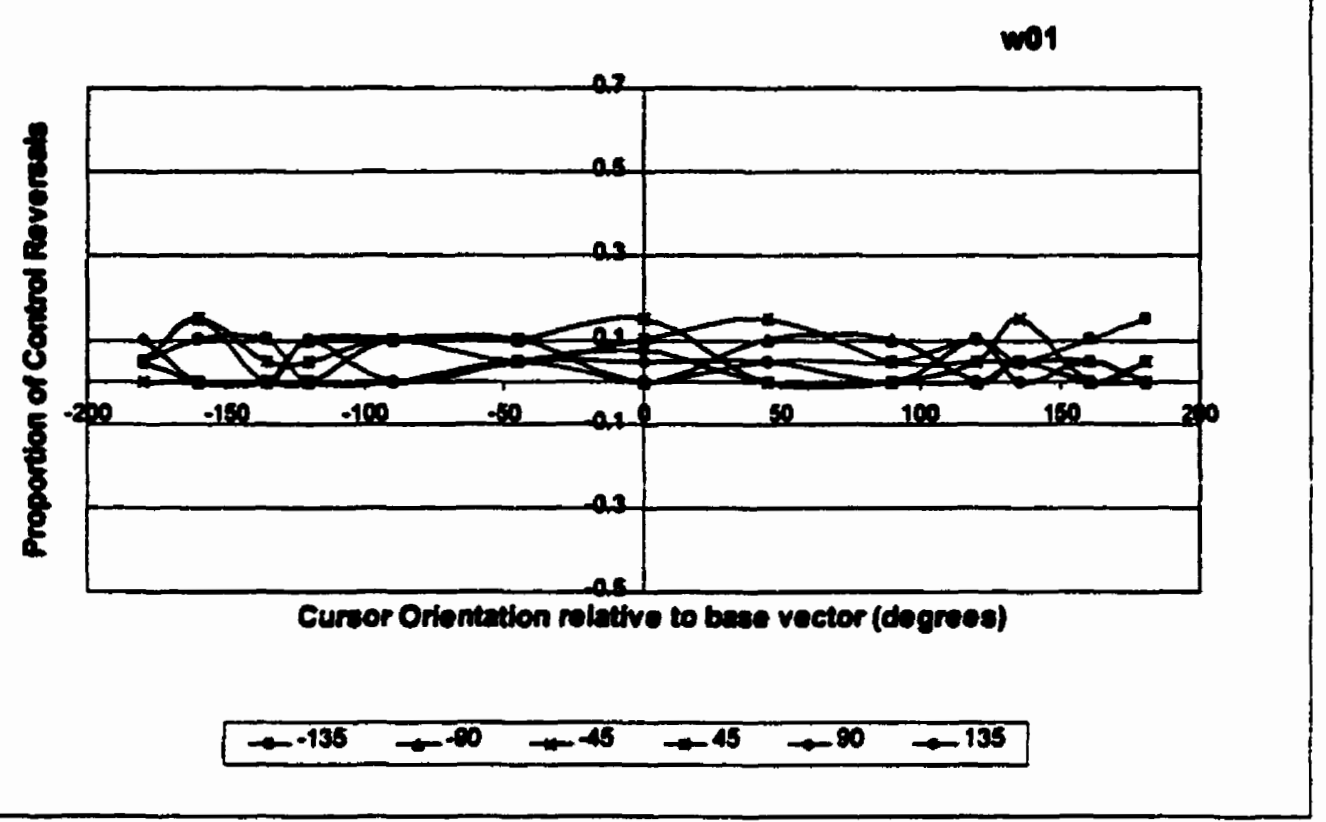

(a)

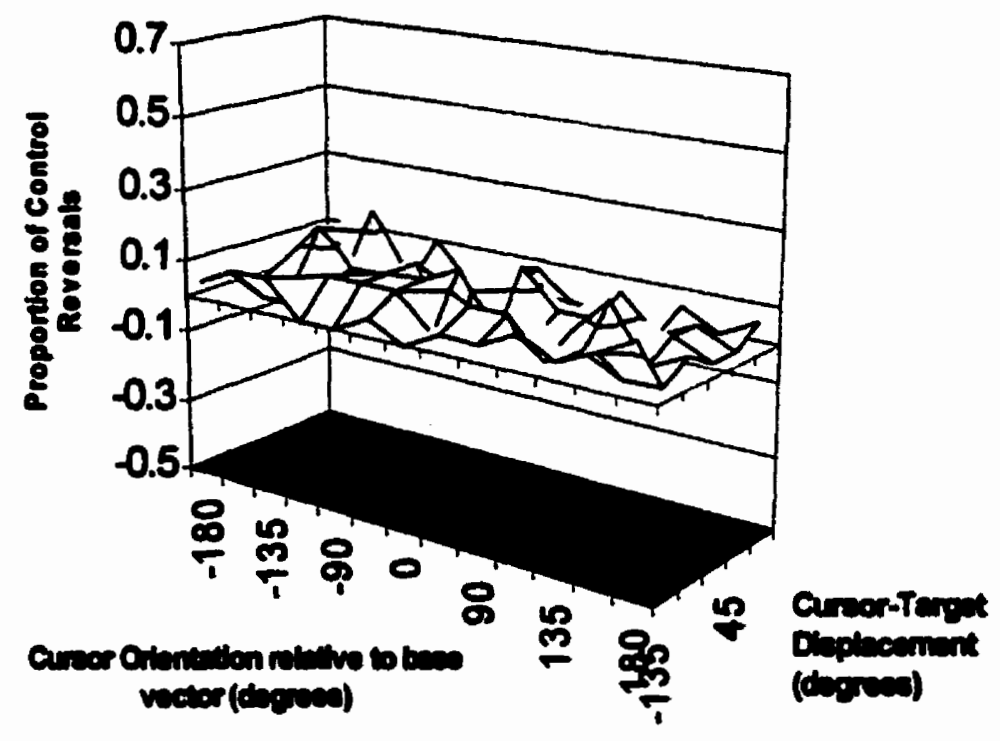

(b)

Figure 41 Cursor Orientation $\times$ Cursor-Tanget Displacement (CO x AD) Interaction for Tether w01 (also representative of the plots for w02, w03, and w99) 


\subsection{Tracking Task Results}

The two sets of tracking data collected for each viewpoint perspective (VP) were separated, since the nature of the dynamic tether for each set differs significantly during tracking tasks. This arises due to the fact that tethering in each case requires the use of tether components along differing degrees of freedom (DOF). Dynamic tethering when given a planar view requires only a 1-DOF tether, whereas tethering when giving a perspective viewpoint requires a 3-DOF tether. It is thus not fair to compare the two directly or combine their data, due to the very different dynamic properties of each.

Tracking performance was measured in two ways. First overall RMS error per tether was calculated, followed by a calculation of RMS error per tether per dynamic-track average target displacement. These measures were made in the time domain, and the next RMS error measures were described in the frequency domain to investigate tether related performance as it relates to the frequency content of the forcing function input track. An RMS error power calculation was then subsequently performed to view the performance measure in terms of groups of frequencies chosen to correspond to the tether natural frequencies.

\subsubsection{Tracking with the Plan Viewpoint}

No significant difference can be detected between each tether condition in terms of RMS error $(F(4,36)=0.298, p=0.877)$. The plot of the data is shown here for the benefit of comparison of these results with that of future studies which may confirm these findings or which may be more sensitive in detecting the hypothesised U-shape trend. 


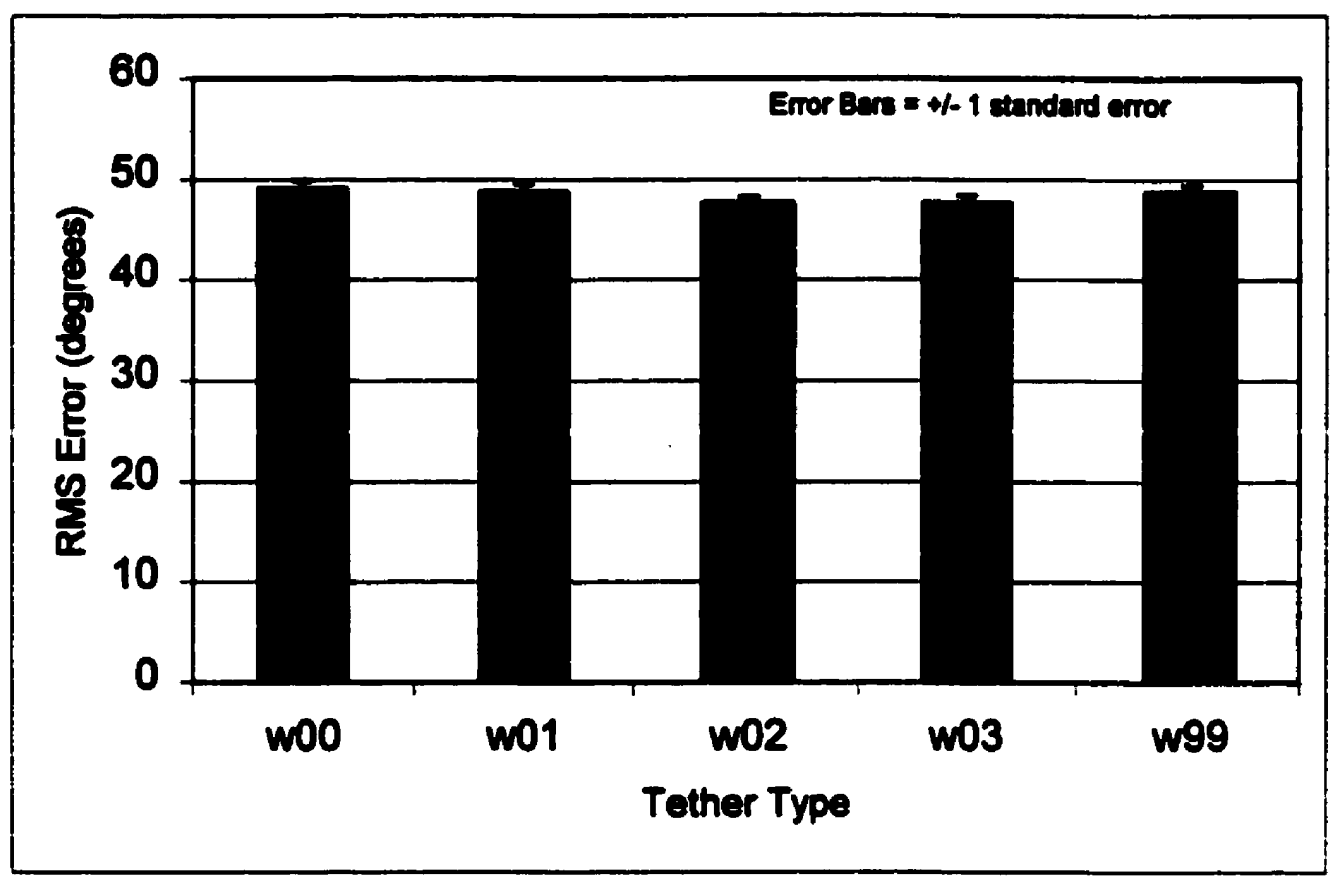

Figure 42 RMS Error per Tether Condition (DT) given a Plan View of the Scenario

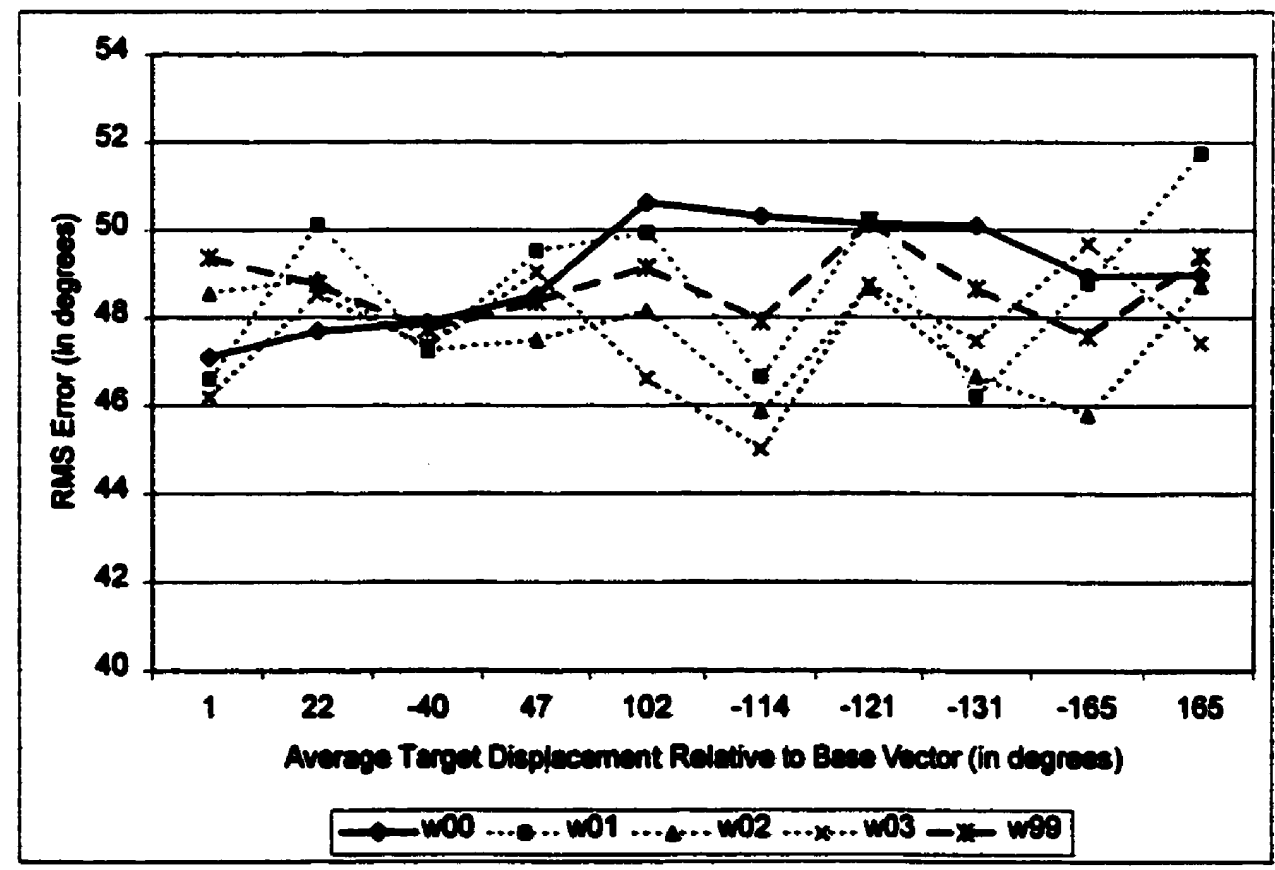

Figure 43 RMS Error plotted against Average Target Displacement (CAD) from the nominal forward facing base vector for each tether treatment 
The effects of tethering is observed as per the results of the dynamic tracking task which are plotted in Figure 43. There is no significantly reliable difference between the curves plotted above $(F(36,324)=1.19, p=0.218)$.

\section{Diagnostic Checking of the Model}

All of the data in this experiment have been analysed to ensure that they do not violate the model assumptions of the analysis of variance. In performing this standard diagnostic checking of the model assumptions, it was found that one assumption was not appropriate, for the tracking data from the plan view experiments. In particular, Subject \#9 exhibited RMS error performance measures that had normally distributed residuals, but the distribution was not centred sufficiently close to zero (see Figure 44). This is a clear violation of the independently and identically distributed in a normal distribution with mean zero and variance $\sigma^{2}$ assumption, denoted as $\operatorname{IIDN}\left(0, \sigma^{2}\right)$, made when using an analysis of variance. The explanation offered for this difference is that the subject was not able to track very well due to the admission that his/her tracking skills were poor and that the task took some getting used to in the beginning phases on each day. His/her non-typical performance was apparently different enough from the general group of test subjects to shift the RMS error frequency upward, thus skewing his/her residuals such that the IIDN $\left(0, \sigma^{2}\right)$ assumption could not be made. 


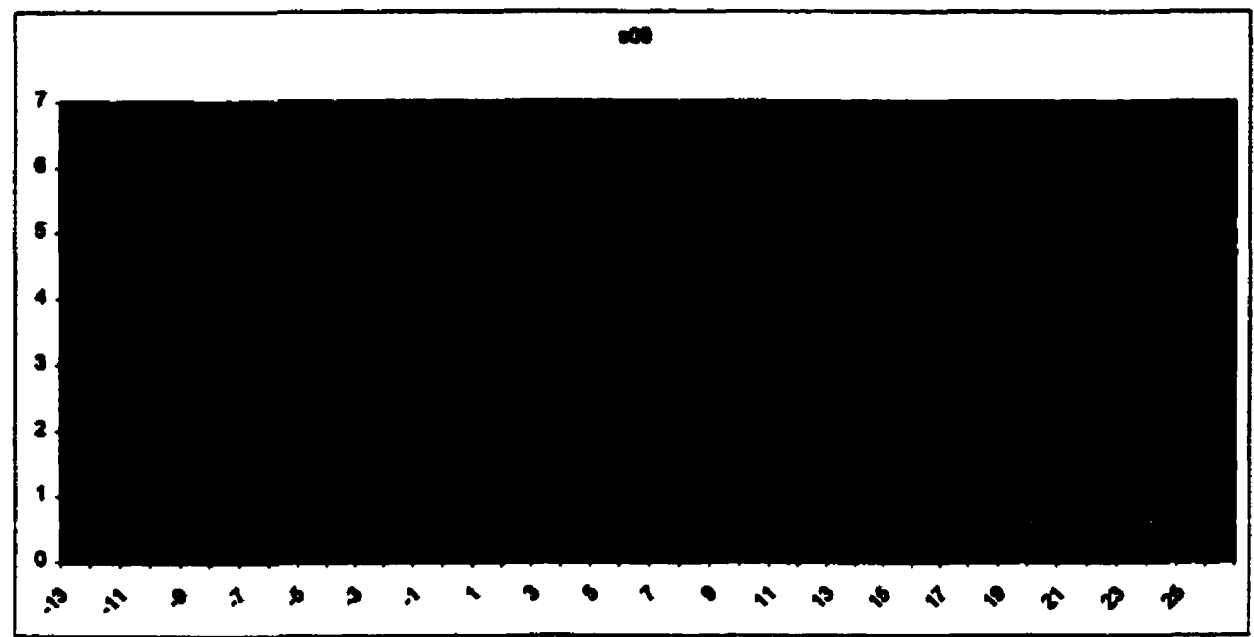

Figure 44 Distribution of Subject 9 's RMS Error Data Residuals

Subject 9's data were then removed from the analysis of the plan view RMS errors and the diagnostic checking and analysis were repeated. Subsequent diagnostic checking revealed no additional violations of the model assumptions. Removal of subject 9's data permitted the detection of significant effects due to a tether $\mathbf{x}$ average target displacement interaction $(F(36,288)=1.541, p<0.05)$. The data were re-plotted and are shown in Figure 45. Curve fitted to the data for w00 tether condition now clearly indicate the dependence of the non-tethered (w00) display performance on the average target displacement from the nominal forward facing base vector. This fit has an $\mathrm{R}^{2}$ value of 0.92. It does not seem that this dependence $\left(R^{2}<0.3\right)$ of tethered (w01 - w99) performance on the average target displacement from the nominal forward facing base vector exists.

The figure below illustrates the superiority of the intermediate tether treatments over the extreme tether treatments. The non-tethered treatment supports good performance at low levels of average target misalignment but it causes increasingly unfavourable 
performance as the absolute magnitude of the average target deviation increases. The tethered displays are not affected by variation in target deviations and thus their curves are relatively flat. However, the more consistent control-display alignment does not come without a cost. As the rigidness of the tether increases, the ability of the operator to perceive the true motion of the display decreases and thus the tracking performance measure suffers. There thus, theoretically, exists an optimal region between the two extreme display modes. The curve fit to the data corresponding to the w03 tether treatment appears to be the closest to the optimal tether's curve, which would be the lowest possible curve resulting from conducting the tracking trials with optimised tether parameters.

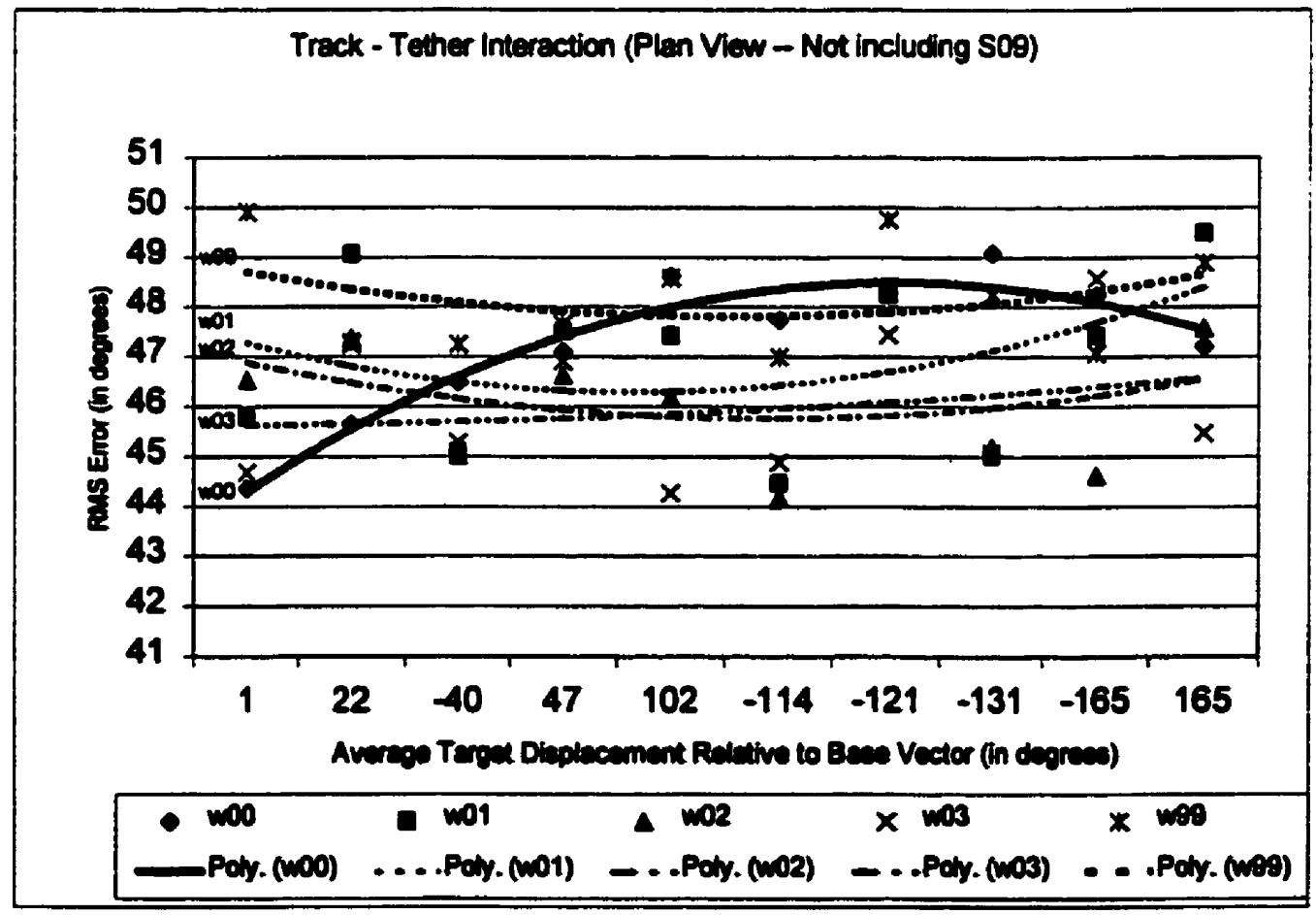

Figure 45 RMS Error plotted against Average Target Displacement from the nominal forward facing base vector for each tether treatment (excluding subject 9's data) 


\subsubsection{Tracking with the Perspective Viewpoint}

The previous analysis was repeated for the tracking tasks performed with the perspective viewpoint and again the same patterns were revealed. Unfortunately, the dynamics of the 3 DOFs of the w02 tether given the perspective view and avatar rotation in the ground plane resulted in extreme and severely lagged camera oscillations. These oscillations caused a complete loss of visibility of the cursor, rendering the interface useless. The data for tether treatment w02 were thus removed from the analysis, and in the study of tracking performance with the perspective viewpoint, discussion is limited to the other four tether conditions (w00, w01, w03, and w99).

\section{RMS Error and the Perspective Tracking Task}

There was no significant difference in the RMS errors measured for each of the four tether treatments $(F(3,27)=0.384, p=0.765)$ in Figure 46 . 


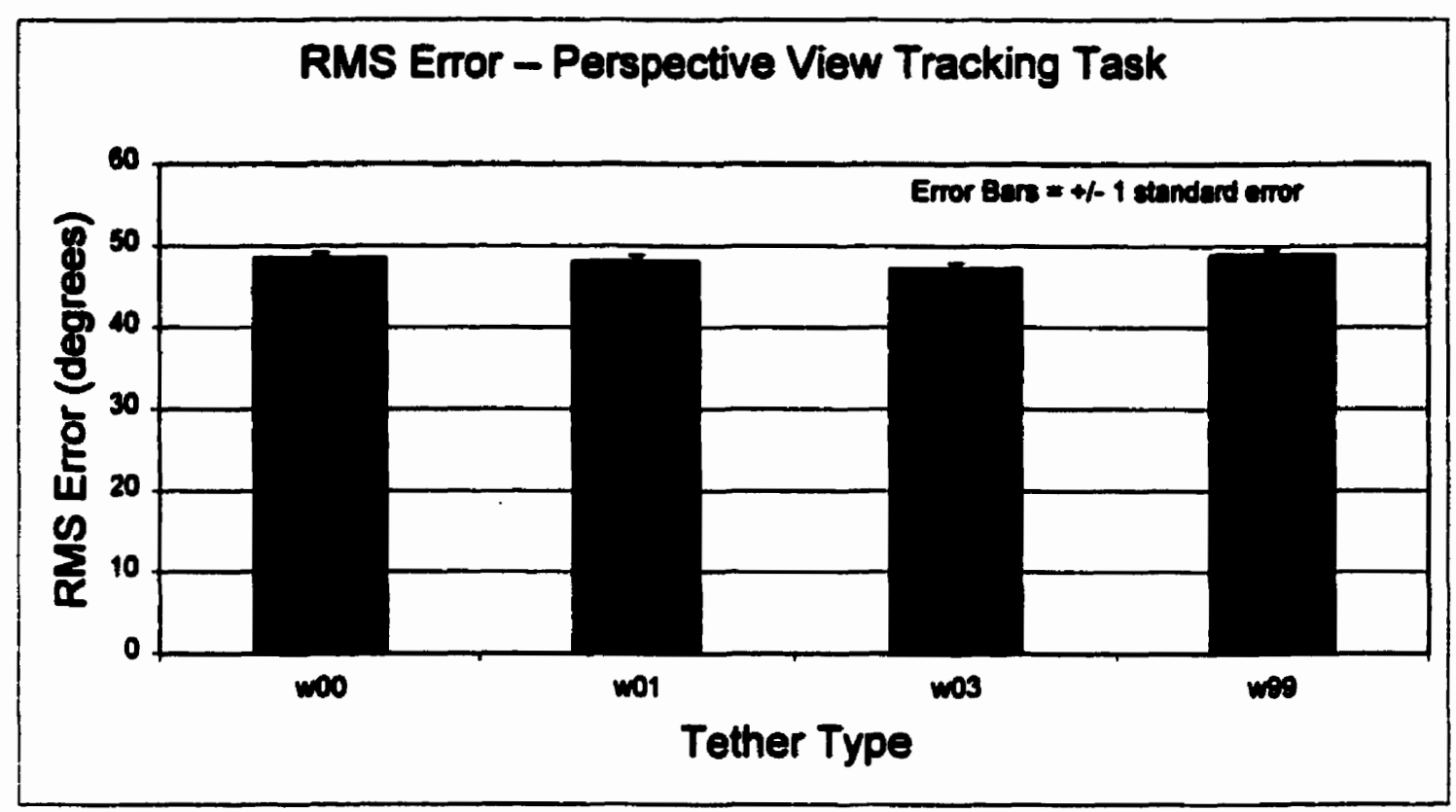

Figure 46 RMS Error per Tether Treatment in the Perspective View Tracking Task

Figure 47 illustrates the relationship between the RMS error for each tether and the average target displacement relative to the base vector. Although analysis reveals that there exists a significant interaction between the plotted curves $(F(27,243)=1.599$, $p<0.05$ ), the variance in the data is extremely noisy, making it difficult to make any reasonable interpretations from the plot. Therefore no conclusion can be drawn about the superiority of any of the tether conditions in the perspective view tracking task. 


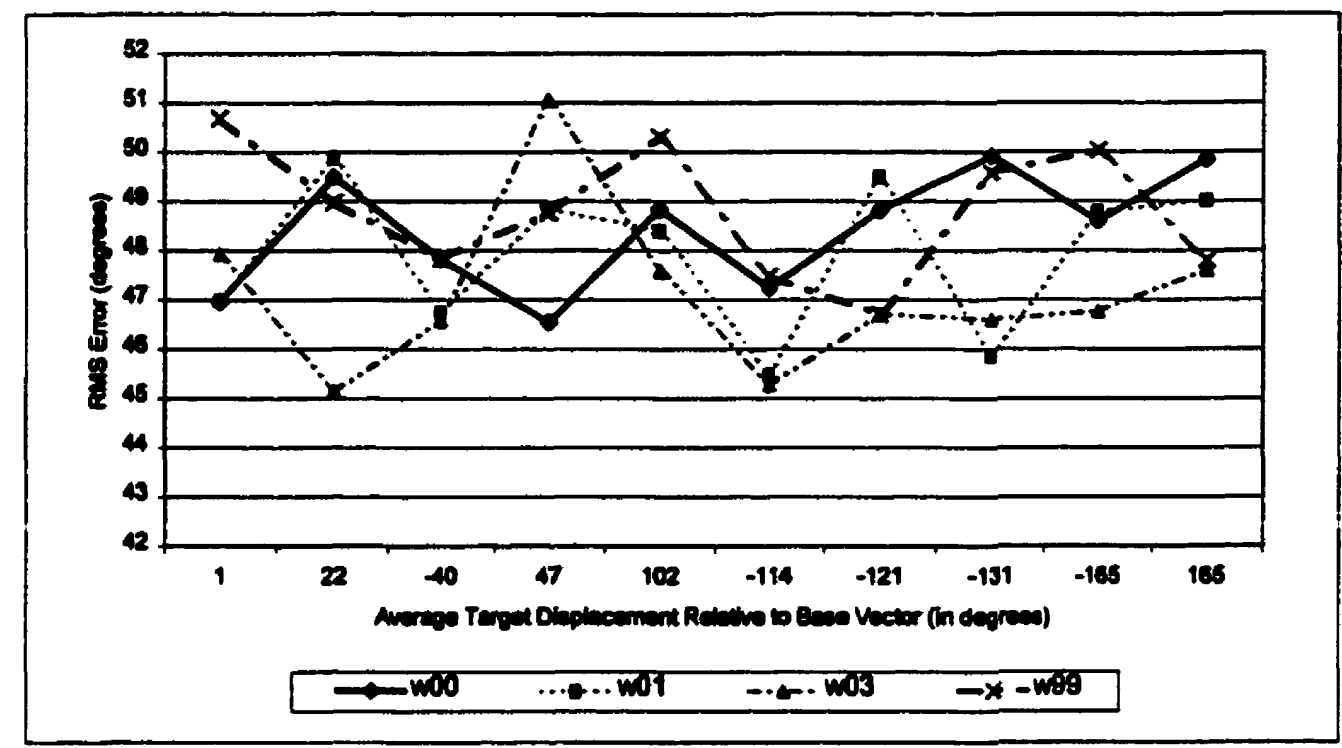

Figure 47 RMS Error plotted against the average Target Displacement for each of the four tether treatments in the Perspective View

\subsubsection{Spectral Analysis of Tethering Effects on Performance}

A spectral analysis was performed on the tracking data collected in the plan view interface arrangement. The following procedure describes the method by which the RMS error amplitude was calculated for each subject and then averaged for an overall measure of performance.

1) The positions of both the cursor and the target were recorded at a sampling period of 20 milliseconds over the entire 30 second tracking task. Each subject performed a total of 10 tracking tasks with each tether treatment.

2) A Fast Fourier Transform (FFT) of both the target and cursor tracks was performed. The FFT transformed the cursor and target time domain tracks into tracks in the frequency domain. Each track was represented by a set of real and imaginary 
components at each frequency, from 0 to $25 \mathrm{~Hz}$ (which is the maximum estimated frequency, given the sampling interval of $20 \mathrm{~ms}$ ) at a resolution of $1 / 30 \mathrm{~Hz}$.

3) The error was then calculated as a measure of the difference between the cursor and target real and imaginary components at each frequency across the 10 tracking trials for each subject. This RMS FFT error represents the average absolute amplitude and phase differences between the target and cursor tracks in the frequency domain. Here RMS FFT error is calculated as follows, where $i$ is a particular track of the forcing function:

$$
\operatorname{Real}(\operatorname{Re}) \mathrm{RMS} \text { FFT error }=\sqrt{\frac{\sum_{i=1}^{10}\left(\operatorname{Re} i_{\text {turget }}-\operatorname{Re} \text { i cursor }\right)^{2}}{10}}
$$

$\operatorname{Imaginary}(\operatorname{Im})$ RMS FFT error $=\sqrt{\frac{\sum_{i=1}^{10}\left(\operatorname{Im} i_{\text {arget }}-\operatorname{Im}_{\text {icursor }}\right)^{2}}{10}}$

4) The amplitude of the complex RMS FFT error sets was then calculated at each frequency as per the following equation:

RMS FFT Error Amplitude $=\sqrt{(\operatorname{Re} R M S \text { error })^{2}+(\operatorname{Im} R M S \text { error })^{2}} \quad$ (equation 9-3)

5) Once the RMS FFT amplitude error per frequency was calculated for each tether treatment and subject, the values were than averaged arithmetically across subjects and are plotted below in Figure 48. 


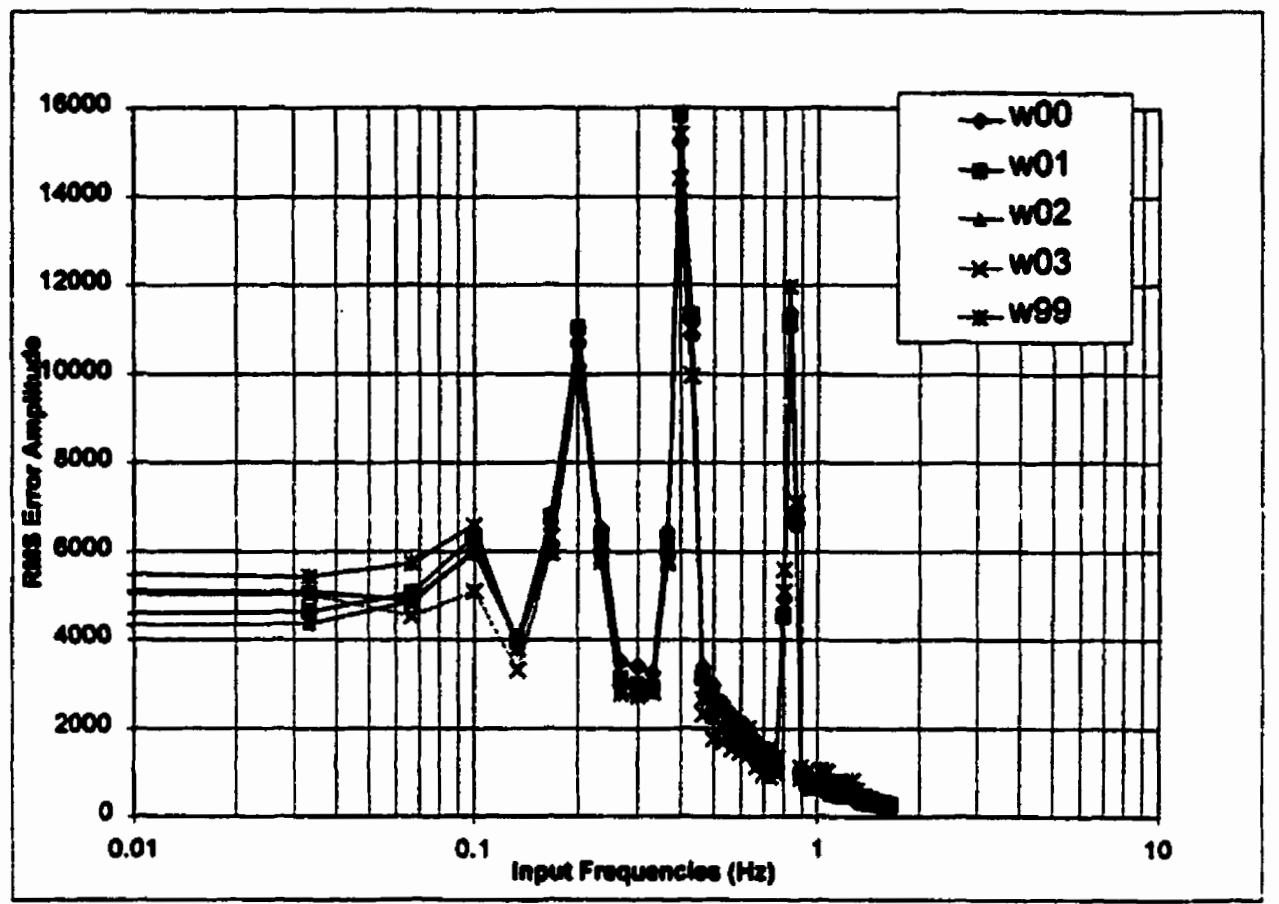

Figure 48 Logarithmic plot of RMS FFT Error Amplitude

Figure 48 is a plot of the resulting averaged values obtained from the fifth step in the spectral analysis calculations listed above. Eleven forcing function frequencies were contained in the target motions: $0.00063,0.00129,0.00265,0.00543,0.01116,0.02292$, $0.04706,0.09665,0.19850,0.40766$, and $0.83723 \mathrm{~Hz}$. (A derivation of these frequencies is provided in Appendix D.) The first six frequencies are too small to be seen in this plot (due to the low frequency resolution of the time window used in the experiment) and this is recognised as a weakness in the design of this experiment, since the spectral power of these hidden frequencies has been distributed among the estimates plotted at $0.0 \mathrm{~Hz}$ and $0.0333 \mathrm{~Hz}$. Spikes in the plot occur at estimates close to the remaining five frequencies that are distinguishable in this plot of $1 / 30 \mathrm{~Hz}$ resolution. The spikes indicate frequencies where large differences between the track frequency composition and the cursor frequency composition occur. The closer the input (cursor) track resembles the target 
track in the frequency domain (i.e. in terms of both frequency content and phase lag/lead) the lower the RMS FFT amplitude error.

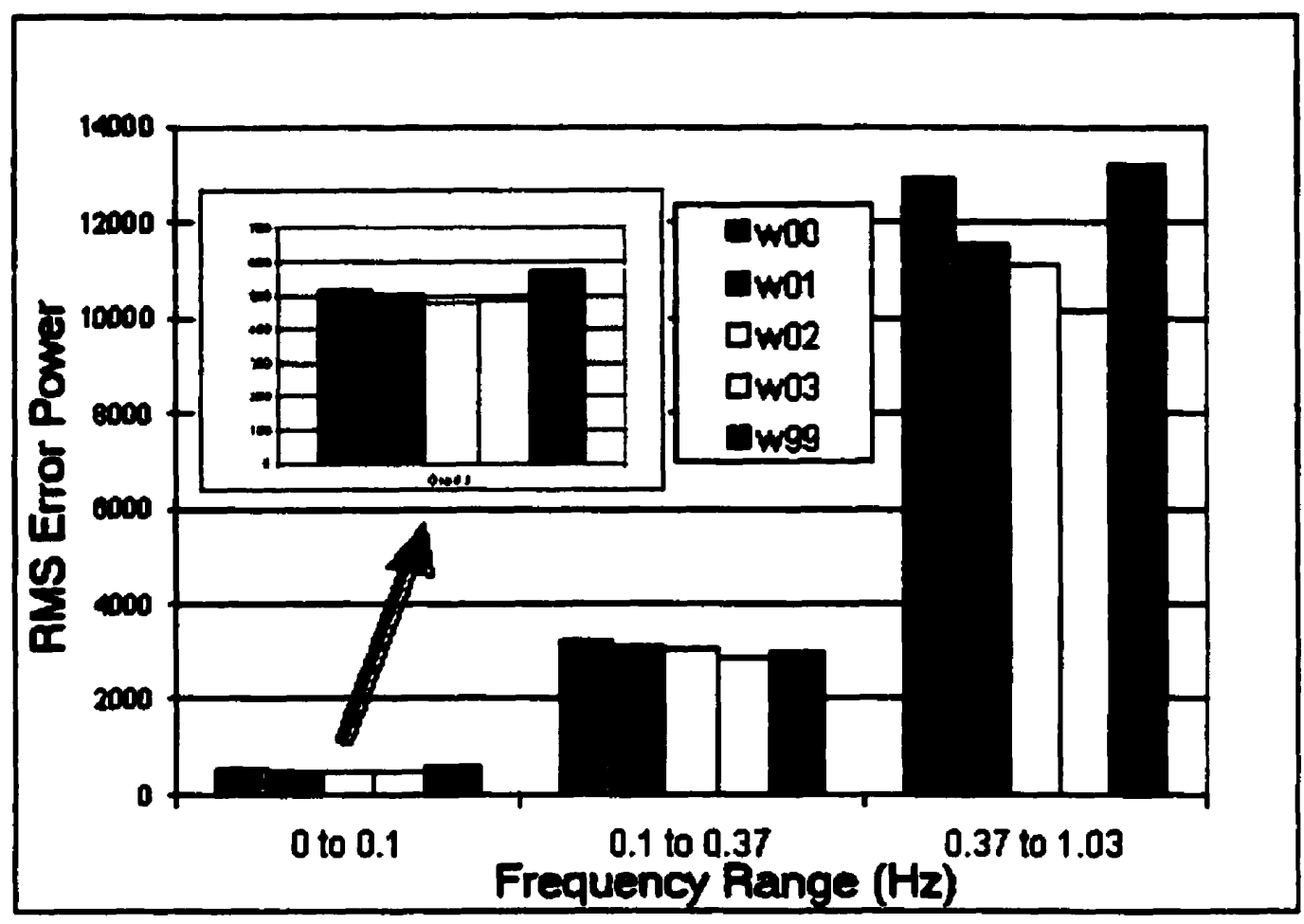

Figure 49 Plot of Spectral Power over three frequency ranges

The bar graphs presented in Figure $\mathbf{4 9}$ were produced by computing the integral of each of the five curves in Figure 48 across three groups of frequency ranges. The plots of the spectral power in these ranges reveal evidence that support the hypothesis that, at very low track frequencies, performance with the tethered displays should prove better than performance with the non-tethered display. Since the advantages of perceiving absolute motion of the target are not present at low frequencies, the non-tethered display (w00) has no advantages to offer over the control-display consistency provided by its tethered counterparts. As the frequency components of the target motion increase, the ability to perceive both absolute motion and relative motion of the position markers becomes 
increasingly important and performance with the rigidly tethered (i.e. compensatory-like w99) display deteriorates and the non-tethered (w00) display's relative performance improves. However, the dynamically tethered displays maintain the beneficial properties of both display types and thus result in better performance measures over the entire range of frequencies, where the w03 tether treatment resulted in the best performance. This may be due to the fact that this tethered display condition did not allow control-display incongruencies to exceed $\pm 90^{\circ}$.

\subsection{Subjective Ratings}

In order to analyse how the operators perceived their own performance with respect to the tethered interface, three different subjective measures of operator-interface compatibility were collected. The following questions were posed to the subjects in a post-experiment questionnaire with verbal explanation supplied as required:

1. How understandable was the displays motion (i.e., was there any difficulty in understanding the cause-effect relationship that drove the display)?

2. How did the interface affect the operator's perception of performance?

3. How did the operator respond to the on-screen motion (i.e., was the motion disorienting or was there no significant effect)?

The data collected via the questionnaire and plotted in Figure $\mathbf{5 0}$ revealed that subjects understood the cause-effect relationships driving the display and this understanding did not vary significantly across the differing tether treatments $(p>0.05)$. However, despite 
the lack of significant differences between the measures of level of understanding of the cause-effect relationship, the plot in Figure $\mathbf{5 0}$ shows that there is no significant difference in subject understanding of the tethered display as the tether was made increasingly more rigid. Also, subjects felt that rigid tethers (tether treatments w03 and w99) degraded task performance more $(F(4,36)=2.235, p=0.085$, i.e. marginally significant) than other less rigid modes (w00, w01, and w02) as illustrated in Figure 51.

Lastly, the most significant finding was that obtained from the measurement of subject reaction to the on-screen motion, the results of which are presented in Figure 52. The general response to the on-screen motion was a feeling of disorientation associated with the very rigid tethers (w03 and w99) to a much greater degree $(p<0.001)$ than that perceived with the less rigid tethers (w00, w01, and w02). A Duncan test revealed significant differences $(\alpha=0.05)$ between group A (w00, w01, and w02) and group B (w03 and w99); however, no other differences can be detected within the two groups of treatments. 


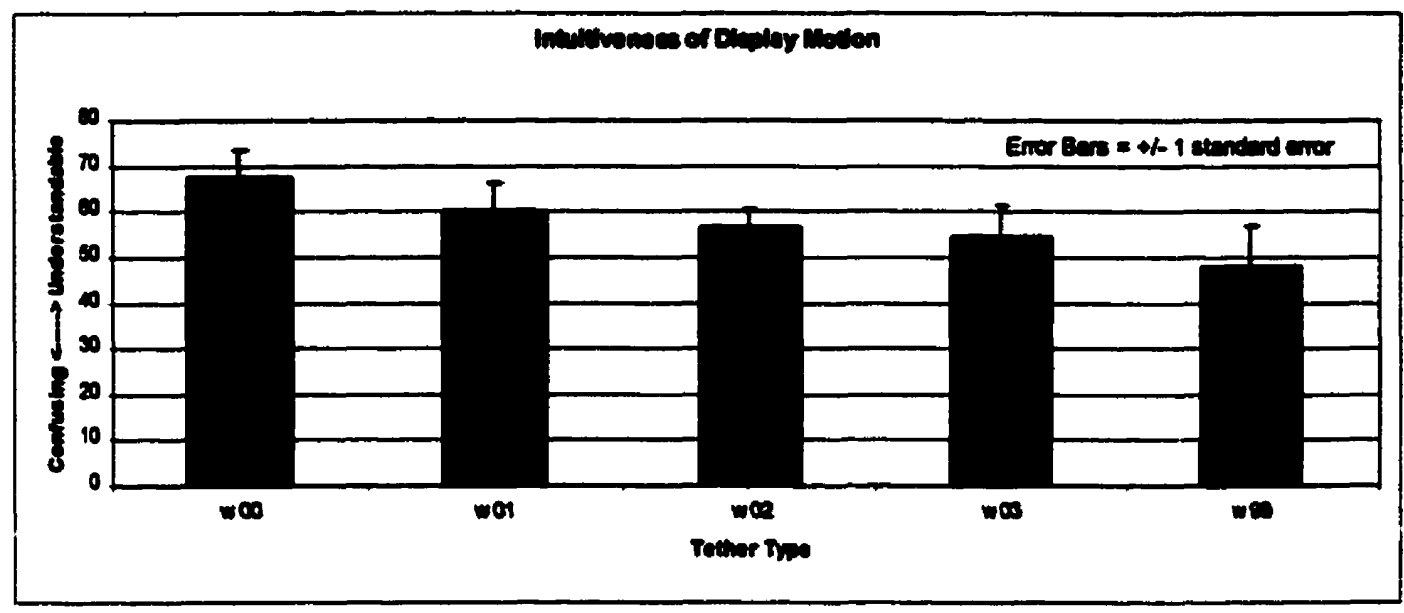

Figure 50 Plot of subject understanding of the eause-effect relationship of the displayed motion

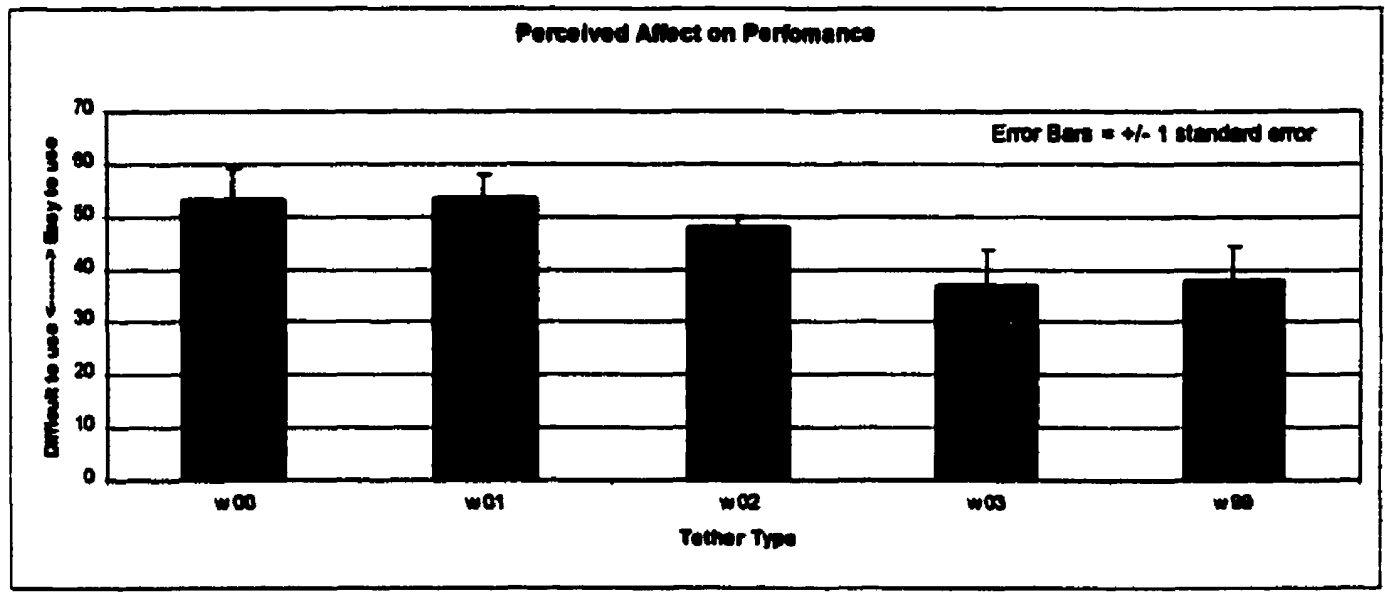

Figure 51 Plot of Subjective measures of pereeived affect of display motion on performance

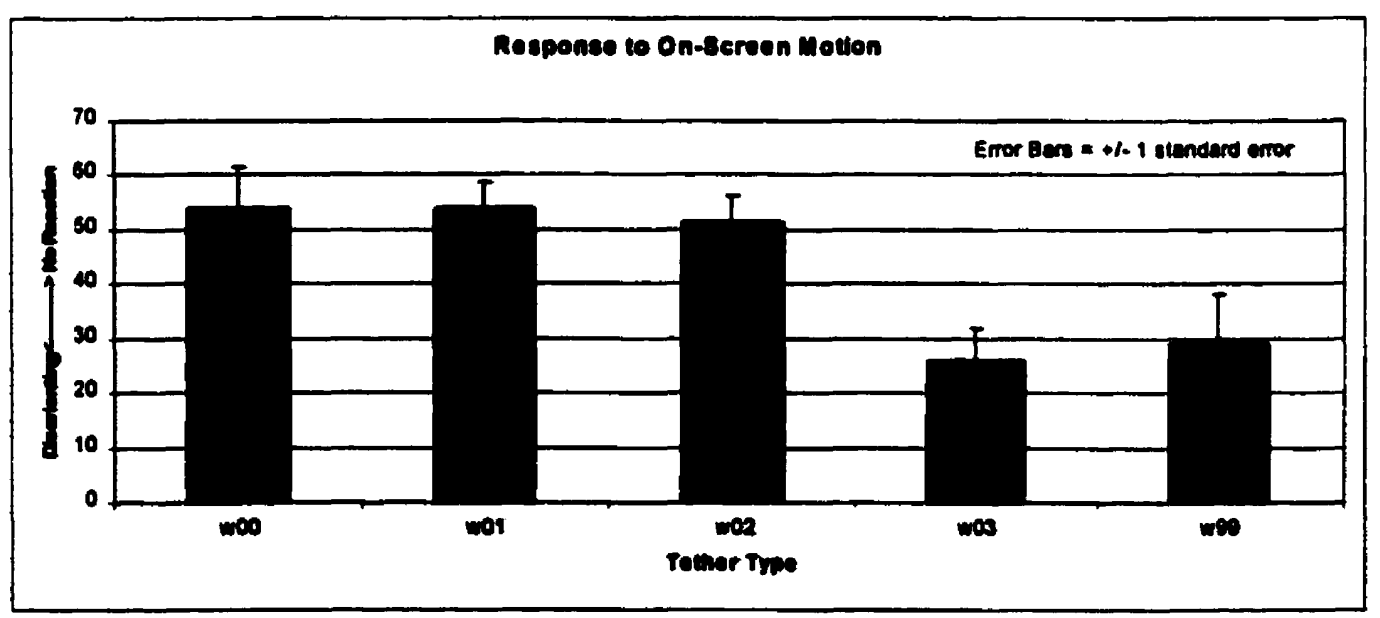

Figure 52 Plot of Subjective rating of response to on-screen motion 


\section{Conclusions}

The experiments presented here closely parallel those of Shepard and Hurwitz (1984). There is a strong similarity between map following and the teleoperation scenario task in this study in terms of the mental rotations performed. The results of the 1984 study and this current study should be similar across parallel aspects. The single most important finding by Shepard and Hurwitz was that the identification of a map-like representation of a turn, as either right or left, takes increasingly longer as the direction of the line going into the turn departs further from upright. The extreme case of a $180^{\circ}$ departure from upright was consistently the most difficult trial to perform. Secondly, they also found that reaction time increased with the magnitude of the preceding turn (see Figure 3).

The results reported in this study are in full agreement with that 1984 study, as well as those of Levine et al. (1982 as cited by Shepard and Hurwitz, 1984). The results here show that reaction times increased as the initial cursor position departs from the forward facing/upright base vector, and secondly, that reaction time also increased with increases in cursor-target angular displacement. Barfield and Salvendy (1985) have also found trends similar to that found here, in terms of mean reaction time. Although, the simple and complex figure mental rotation times at $180^{\circ}$ of angle rotation were faster than that of $120^{\circ}$, the results obtained with a figure of moderate complexity did not reveal the same decrease in reaction time. This finding corresponds with that resulting from this study possibly because the imagery used in the virtual reality display is of a level of complexity equivalent to that deemed moderate in Barfield and Salvendy's study. Bernotat (1970) 
also discusses why the human operator's inability to compensate for display rotations causes tracking errors to increase considerably at $90^{\circ}$ and $270^{\circ}$ rotation angles.

The results of past studies and those of the present study indicate that mental transformations cause increases in reaction time and increases in the frequency of performance errors during tracking tasks. In addition, this study also suggests that implementing an appropriate dynamic tethering algorithm can mitigate these deficiencies.

Many conclusions may be drawn from the large amount of data collected and analysed in this investigation. The conclusions based on the docking, tracking, and subjective evaluations are summarised in the following sections.

\subsection{Docking Conclusions:}

Dynamic tethering does not increase nor decrease the time required to complete the given docking tasks, nor does it affect the overshoot measures associated with those tasks. Perspective views (when compared to planar views), however, increase the completion time associated with the docking tasks performed in this experiment.

Large control-display incongruencies $\left(\nexists \pm 90^{\circ}\right)$ induce a high frequency of control reversals in operator control when a non-tethered teleoperation display is provided. The tethering technique does, however, decrease the probability of operator control reversals by keeping the control-display congruency within acceptable limits. Control reversals are more likely to occur as the angular distance between the cursor and the target approaches 
the maximum of $\pm 180^{\circ}$, due to increasing difficulty in determining the correct direction of rotation.

Without a tether connecting the controlled object to the viewpoint, control-display misalignments are permitted to occur. When they do occur, the mean reaction time of the operator is proportional to the magnitude of the incongruency. Tethering, in general, reduces the time required to determine the required direction of input. This reduction in reaction time results from the tether's ability to minimise the magnitude of controldisplay misalignment presented to the operator.

\subsection{Tracking Conclusions:}

Dynamic tracking of a target requires an operator to perform much of the same operations required for navigation and manipulation within the teleoperation environment. Dynamic tethering improves performance slightly in terms of RMS error when the target track causes a large degree of control display incongruencies and/or when it contains fairly high frequency components.

Analysis of tethered performance over the average angular position of a target revealed that, without a tether, mean RMS errors increase with increasing mean angular position (where a zero average angular position refers to a track oscillation that is centred about a forward/upward facing base vector that is aligned with the operator's controls). Thus, control-display incongruency appears to be the primary reason for poor performance when using a non-tethered display. 
However, when the display is tethered, performance becomes independent of target position and becomes a function of tether rigidity. As tether rigidity increases, performance improves at first. Then, when the optimal tether rigidity is reached, further increases in stiffness cause performance to deteriorate. At high track frequencies the more flexible tethers allow the display to venture out of congruence with the control to a greater degree than their more rigid counterparts. Increasing the stiffness of the tether prevents the display from rotating into detrimental positions. But, as tether stiffness is increased beyond the optimal value, the decreasing magnitude of object-viewpoint relative motion becomes increasingly harmful to task performance as the usable information required for optimal tracking is reduced. In this study, the target signal produced by the forcing function was best tracked when the w03 tether condition was implemented. Further study should be devoted to examine the relationship between the natural frequency of the tether and the characteristics of the forcing function.

A spectral analysis indicated that at low frequencies tracking performance is slightly better with less stiff tethers (i.e. tethers with low natural frequencies) than rigid tethers. As the track frequency component increases optimal performance shifts to systems that implement tethers with higher natural frequencies. At the top track frequencies, performance with a pursuit type I display (i.e., w00) is definitely better than pursuit type II displays (i.e., w99). However, performance with dynamically tethered displays with medium to high natural frequencies (i.e., w02 and w03) is improved over the two traditional displays (i.e., w00 and w99). Thus, it seems that the optimal tether 
implementation for any tracking task is dependent on the frequency content of the forcing function.

\subsection{Conclusions based on Subjective Evaluations:}

Increases in the stiffiness of the dynamic tether make the display increasingly difficult to interpret and operators will have a negative view of its effect on their performance. Thus, the tether with the lowest acceptable natural frequency within a group of dynamic tethers that result in the best tracking and docking performance should be used over those with higher natural frequencies, to minimise operator confusion and improve the operator's perception of their performance. Of the tethers used in this experiment performance was best with w02 and w03, but, in accordance with the findings of the subjective evaluations, the w02 dynamic tether should be implemented over the w03 tether.

The results of this experiment may be used in the design of control systems to aid the navigation and control of remote devices. The reduction of reaction time and control reversals may mean the difference between a critical rescue and a critical error in applications such as endoscopic surgery where control-display incongruencies are commonplace. Such an algorithm with its tracking advantages, such as its decrease of RMS error, may also be used in advanced control paradigms in which a remotely controlled robot and its environment are modelled in a mixed reality or virtual reality display. The chances of an error occurring during such a task will be reduced by implementing the findings of this study. In space robotic applications it is very possible that the task environment can be modelled (e.g. a space station or space shuttle) and 
control errors in this environment can cause an important piece of equipment to fly off into space or worse cause a catastrophic event to occur. In some less risky operations, the technique may be applied to simulated land navigation as tool to improve the user's mental mapping of the environment and in video games to improve the sense of presence by taking advantage of the diminished disorientation associated with these displays over the highly disorienting traditional rigidly tethered displays. 


\section{Recommendations}

In conducting these experiments much has been learned about performance effects of dynamic tethering, but also more questions have been raised which may become the basis for further research in this domain. Modifications to the experimental design, the dynamic tether model, and the implementation of the algorithm are some aspects that may be worth investigating in future studies.

In this experiment a within-subjects factorial design was used, in order to collect comparative subjective data to evaluate each of the display techniques with respect to the others. In his book, Poulton (1974) discusses the issues of negative and asymmetric transfer of training with respect to compensatory and pursuit display comparisons. Apparently, performance with pursuit displays suffers if the operator has recently used a compensatory display to perform the same task, while performance with compensatory displays benefits from prior use of pursuit displays. Future work may investigate the same issues discussed in this paper but with a between-subjects factorial design. This design would then also allow for each subject to practice the task to a greater extent, in an attempt to eliminate the ramping effects of each operator's learning curve. In addition, a study might be conducted to investigate whether negative or asymmetric transfer of training issues indeed apply in the comparison of pursuit type I versus pursuit type II (or compensatory-like) displays.

On a similar note, it seems that the presence of a background texture in the scene provides more or better information during task execution than no background texture at 
all. The presence of a background may not significantly improve task performance over that using a traditional compensatory display where only the error is visible and no backdrop information is provided. Future research may attempt to compare the two within the context of dynamic tethering.

Research by Corballis and McLaren (1982, as cited by Finke and Sheppard, Chapter 37 in Boff et al., 1986) indicates that observing patterns undergoing actual rotation has a biasing effect on subsequent mental rotation. This may be one explanation for the lack of experimental resolution in illustrating differences in reaction times based on the magnitude of cursor rotation. It is possible that (in the docking task) the rotational direction of the preceding trial may have affected the direction of mental rotation in the subsequent trial, despite the requirement to rotate the cursor through the smallest angle. If subjects do not consistently perform mental rotations through the smallest angle, then the variance in the measured reaction times may increase, thus requiring tests of high sensitivity in order to detect any significant effect of angle on the time to action. One solution may be to provide task scenarios that induce mental rotations in the same direction. That way the direction of rotation in one trial should have no bearing on the rotation required in the following trial.

The tether model may also be modified from a simplistic spring-damper system to a more involved PID control loop. Future research may look at the optimisation of the controlloop in Figure 17 in order to attempt to predict performance based on the task and tether dynamics. Using PID controllers to model the object-camera link may result in a closer 
to optimal tethering system with less control restrictions. PID control theory may be used to achieve a deadbeat response, or another possibly more suitable response, to model the dynamic tether. Research may be performed in this respect for something as simple as investigating the implications of varying the damping ratio or the more complex optimisation of the human-machine control loop.

Lastly, on-the-fly adjustment of the dynamic tether parameters was beyond the scope of this paper, but could become the topic of future research. The manipulation of dynamic tether parameters in the time domain may prove to benefit the user. However, the implementation of such a system may require 'smarter' computing ability than is currently available.

In all, if the need makes itself apparent, there is seemingly much that can be gained from the use of dynamic tethering and much to be gained from continued research in the field. 


\section{Glossary of Terms}

centricity

the extent to which a human observer's viewpoint is removed from the "ownship", that is, from the nominal viewpoint with respect to the viewer's own avatar, or own vehicle, or own manipulator within the task space.

compatibility principle of congruence: with respect to location of controls, principle that the spatial arrangement of a set of two or more displays should be congruent with the arrangement of their controls; with respect to orientation of controls, the spatial orientation of a display should be congruent with the arrangement of its controls. (Wickens and Carswell, 1997)

compensatory display a display which conveys error information about a tracking task that has only one moving index. The direction and distance of this moving index relative to a fixed reference represents the error to be countered.

Compensatory-like display: see pursuit (type II) display.

congruency

state of one-to-one correspondence between displays and associated controls

control reversal

a control movement in a direction opposite to that intended by the operator

cursor-referenced compensatory display: a display within which a fraction of the cursor symbol's motion is removed and placed on the target symbol, with the effect that the cursor deviates less from the reference point.

display-control compatibility a principle that dictates that the location of a control should correspond to the location of the display with respect to the principle of collocation or the principle of congruence and that the movement of the control and display should be congruent in form and direction with respect to stereotypes and the congruence of movement axes.

egocentric

looking at everything as it affects oneself or ownship from an internal local or one's own point of view; a view from within one's representation located at the nominally defined viewpoint.

egocentred reference frame (MRT): the reference frame that corresponds to the forward view of the world and is established by a pilot's forward view out the cockpit (Aretz, 1991). 
egomotion

enantiomorphic

exocentric

inside-out display

frame of reference (FOR) a defined reference point or viewpoint

imagery (analogue) model theory of mental rotation which states that people can represent visual information in memory through images, which bear a 1:1 correspondence to the physical representation of the object.

an external locale; a view of one's representation away from the nominally defined viewpoint

a display which corresponds to a bird's eye view of a situation

intention-response-stimulus (IRS) compatibility: a set of rules which govern how the operator's intention to respond maps to the actual response, and then to the display signal.

heading-fixed display

a display that constantly depicts ego-position from behind its momentary direction of travel (Wickens, 1998).

induced motion

the perceived motion of objects that are not actually moving, whenever other objects are moving instead

kinesthesis

literal images

mediation

miscorrespondence

object-relative motion

outside-in display sense of motion; awareness of motion of one's body and/or parts of one's body

very accurate images

reduction of the need for mental transformation and the relaxation of the requirement to determine the direction of rotation before applying a control input to the system

the inability or difficulty in mapping from eye or other sensor to mind, and then to hand

motion information that is available from the relative changes in the position of retinal images of objects (Boff and Lincoln, 1988).

a display which corresponds to an egocentric view of a situation 
ownship

position fixed display

position slaved display viewer's own nominal avatar, vehicle or manipulator within a task space

principle of compatibility of display orientation: the orientation and spatial ordering of a display should be consistent with those of its associated mental representation

principle of compatibility of display movement: the direction of movement of the moving part of the display should be consistent with user expectations

principle of conceptual compatibility: the layout and operational methods of controls should be consistent with user expectations or population stereotypes

principle of direction compatibility or principle of movement compatibility the indicator of a display should move in the same direction as its control.

principle of the moving part: refers to the notion that the moving part of a display should conform to the mental model of the operator by responding in the same direction as the movement resulting from an outside-in view of ownship.

proprioception

the ability to receive stimuli about posture and body position from within an organism, or receiving these stimuli, or concerned with receiving such stimuli.

propositional theory theory of mental rotation stating that visual information in memory is transformed into propositions, where a proposition is the smallest unit of knowledge that can stand as a separate assertion.

Proximity Compatibility Principle (PCP) "In general, the principle holds that ... indicators or displayed data values that are conceptually related, or that need to be used in combination, should belong to the same perceptual group. In short, related information should be perceptually proximate." (Bennett, Magy, and Flach, in chapter 20 of Salvendy, 1997).

proximity of movement stereotype: with any rotary control, the are of the rotating control element closest to the moving element of the display is assumed to move in the same direction as that display element. 
paychological refinctory period (PRP): refers to the delay between responses to stimuli when two or more stimuli are presented close together in time. The delay of a second response is due to a slowing effect of having had to respond to a recent prior stimuli.

pursuit (type I) display

pursuit (type II) display

or

compensatory-like

display a display with at least two moving indices, one representing the desired position or course (i.e. a target marker) and the other the actual position (i.e. a cursor). Both symbols move against a common scale or background reference system.

representational processing: Processing of spatial information from neural activities which explore and consult internal representations of the physical environment, that are embodied in memory stores. These include mental representations of local maps, spatial relationships of routes relative to landmarks, relative positions between objects, and the position of the body itself in relation to its stationary environmental frame." (Paillard, 1991)

reversal errors

sensorimotor processing

situational awareness

spatial reasoning a display with two indices but only one of which moves with the other acting as a reference, one represents the desired position or course (i.e. a target marker) and the other the actual position (i.e. a cursor). Both symbols are displayed against a common moving scale or background.

control input in the wrong direction; the result of misinterpreting an instrument's indication and making a control movement that aggravates rather than corrects the undesirable condition (Johnson and Roscoe, 1972).

Processing of spatial information mainly due to that part of the physical world to which the organism is attuned by virtue of its basic sensorimotor apparatus. Local sensorimotor instruments entertain direct dialogues with that world and thus contribute to the continuous updating of a body-centred mapping of extra-corporal space where objects are located and to which actions are directed. (Paillard, 1991)

"perception of the elements of the environment within a volume of time and space, the comprehension of their meaning and the projection of their status in the near future" (Endsley, 1997)

the ability to make inferences, e.g. figure rotation, based on the information contained within a spatial representation (Perricone, 1983) 
spatial representations

stimulus response compatibility refers to the "naturalness" of the relationship between a stimulus and the response with which it is associated

subject relative motion

motion that gives the observer information about the absolute motion of the object relative to the observer and is drawn from retinal-image motion and from information about movement of the observer's own head and eyes (Boff and Lincoln, 1988).

target referenced compensatory display: a display within which a fraction of the target symbol's motion is removed and placed on the cursor symbol, with the effect that the target deviates less from the reference point.

telekinesthesis

teleoperntor

telepresence

teleproprioception

tethered display

third-person video game kinesthesis at a distance; ability to sense motion and/or dynamic constraints within a remotely controlled system

"A machine [or mechanism] that extends a person's sensing and/or manipulating capability to a location remote from that person." Sheridan (1994)

impression of remote presence, due to perception of remote environment and ability to influence it

proprioception at a distance; human operator's sensing and keeping track of the location and orientation of the teleoperator and its appendages relative to its base, to each other, to external objects, and to the operator's own body if necessary. (Sheridan, 1994)

a viewpoint that positions the user's vantage point behind the representative icon, allowing a wider view of the environment, but not to the same extent as the exocentric display. Furthermore, the tethered perspective continues to follow the representative icon as it turns and travels through the environment, thus allowing the user a view of the environment that requires no mental rotations to interpolate position or attitude (McCormick et al., 1998).

a video game in which the user has a bird's-eye-view of their actors or avatars (i.e., the representation of themselves within the virtual world), in contrast to games in which the user display provides a first-person, egocentric view of the game space through the eyes or the window of the avatar 
the characteristic of a system design that influences an operator's ability to extract and integrate information across several displays. A concept that provides a solution to the problem of becoming cognitively lost as a display user traverses through multiple displays pertaining to different aspects of the same system or database. It involves the following four basic guidelines: using consistent representations, using graceful transitions, highlight anchors, and display continuous world maps. (Wickens, 1992)

Visuospatial scratchpad the notion that spatial working memory represents information in an analogue spatial form, often typical of visual images.

world centred reference frame (WRF): the reference frame that corresponds to a map view of the world and is established by a visually presented map in which the traditional canonical alignment is north up in Aretz, 1991. 


\section{Appendix A - Experiment Design}

The current experiment was run using the blocked design illustrated below. Five dynamic tether treatments (w00, w01, w02, w03, w99) were randomised across blocks (days) using a Latin square design. The design was then replicated and counter-balanced across the ten subjects. Furthermore, to study the affects of viewpoint on the measures of interest that variable was counter-balanced across the trials ( $\mathbf{a}$ - above/planar view, $\mathbf{b}$ behind/perspective view). Then finally, the five task scenarios (the different mixes of docking and tracking tasks: $\mathrm{rl}, \mathrm{r2}, \mathrm{r} 3, \mathrm{r} 4$ and $\mathrm{r} 5$ ) were replicated and randomised for each subject. The design described is illustrated in the table below.

Within-Subject

Design

\begin{tabular}{|c|c|c|c|c|c|c|c|c|c|c|c|}
\hline 1 & 2 & 3 & 4 & 5 & 6 & 7 & 8 & 9 & 10 \\
\hline 1 & $w 00, a, r 3$ & $w 01, b, r 5$ & $w 02, a, r 1$ & $w 03, b, r 4$ & $w 99, a, r 5$ & $w 00, b, r 5$ & $w 01, a, r 2$ & $w 02, b, r 2$ & $w 03, a, r 2$ & $w 99, b, r 2$ \\
\hline & $w 00, b, r 2$ & $w 01, a, r 2$ & $w 02, b, r 2$ & $w 03, a, 42$ & $w 99, b, r 4$ & $w 00, a, r 3$ & $w 01, b, r 4$ & $w 02, a, r 3$ & $w 03, b, r 4$ & $w 99, a, r 3$ \\
\hline 2 & $w 01, a, r 4$ & $w 02, b, r 1$ & $w 03, a, r 2$ & $w 99, b, r 3$ & $w 00, a, r 3$ & $w 99, b, r 2$ & $w 00, a, r 5$ & $w 01, b, r 1$ & $w 02, a, r 3$ & $w 03, b, r 4$ \\
\hline & $w 01, b, r 4$ & $w 02, a, r 5$ & $w 03, b, r 3$ & $w 99, a, r 2$ & $w 00, b, r 5$ & $w 99, a, r 2$ & $w 00, b, r 2$ & $w 01, a, r 4$ & $w 02, b, r 1$ & $w 03, a, r 1$ \\
\hline 3 & $w 99, a, r 3$ & $w 00, b, r 3$ & $w 01, a, r 4$ & $w 02, b, r 3$ & $w 03, a, r 3$ & $w 01, b, r 1$ & $w 02, a, r 5$ & $w 03, b, r 4$ & $w 99, a, r 2$ & $w 00, b, r 3$ \\
\hline & $w 99, b, r 5$ & $w 00, a, r 4$ & $w 01, b, r 4$ & $w 02, a, r 4$ & $w 03, b, r 4$ & $w 01, a, r 4$ & $w 02, b, r 1$ & $w 03, a, r 5$ & $w 99, b, r 5$ & $w 00, a, r 2$ \\
\hline 4 & $w 02, a, r 2$ & $w 03, b, r 3$ & $w 99, a, r 5$ & $w 00, b, r 5$ & $w 01, a, r 2$ & $w 03, b, r 1$ & $w 99, a, r 3$ & $w 00, b, r 2$ & $w 01, a, r 5$ & $w 02, b, r 5$ \\
\hline & $w 02, b, r 1$ & $w 03, a, r 1$ & $w 99, b, r 1$ & $w 00, a, r 1$ & $w 01, b, r 1$ & $w 03, a, r 5$ & $w 99, b, r 4$ & $w 00, a, r 5$ & $w 01, b, r 4$ & $w 02, a, r 5$ \\
\hline 5 & $w 03, a, r 1$ & $w 99, b, r 4$ & $w 00, a, r 3$ & $w 01, b, r 5$ & $w 02, a, r 1$ & $w 02, b, r 4$ & $w 03, a, r 1$ & $w 99, b, r 3$ & $w 00, a, r 1$ & $w 01, b, r 4$ \\
\hline & $w 03, b, r 5$ & $w 99, a, r 2$ & $w 00, b, r 5$ & $w 01, a, r 1$ & $w 02, b, r 2$ & $w 02, a, r 3$ & $w 03, b, r 3$ & $w 99, a, r 1$ & $w 00, b, r 3$ & $w 01, a, r 1$ \\
\hline
\end{tabular}




\section{Appendix B - Results of ANOVA and Multi-Factor Contrast Tests}

\section{B1 - Docking Experiments}

F-table for the Analysis of Variance (Dependent: Log(Completion Time))

\begin{tabular}{|c|c|c|c|c|c|}
\hline source & $\frac{\text { sounrea }}{2879.772}$ & $\frac{d}{1}$ & $\frac{\text { Squan }}{2679.772}$ & $\frac{\text { F-Value }}{1469.884}$ & $\begin{array}{r}\text { P-Value } \\
0.0001\end{array}$ \\
\hline SURJECT & 16.407 & 9 & 1.823 & & \\
\hline TETHER & 1.2828 & 4 & 0.3207 & 1.545 & 0.210 \\
\hline TETHER SUBJECT & 7.4711 & 38 & 0.2075 & & \\
\hline VIEW & 0.9448 & 1 & 0.948 & 6.795 & 0.028 \\
\hline VIEWSUEJECT & 1.2514 & 9 & 0.139 & & \\
\hline TETHER VEW & 0.7168 & 4 & 0.1792 & 2.982 & 0.032 \\
\hline TETHERVEW'SUBJECT & 2.1635 & 38 & 0.0001 & & \\
\hline CURSOR & 0.3734 & 12 & 0.0311 & 1.632 & 0.093 \\
\hline CURSOR 'SUEJECT & 2.08 & 108 & 0.0191 & & \\
\hline TETHER'CURSOR & 0.7874 & 48 & 0.0168 & 0.897 & 0.671 \\
\hline TETHER'CURSOR'SUBJECT & 8.0041 & 432 & 0.0186 & & \\
\hline VIEWCURSOR & 0.1478 & 12 & 0.0123 & 0.81 & 0.640 \\
\hline VIEW'CURSOR'SUBJECT' & 1.843 & 108 & 0.0152 & & \\
\hline TETHER'VIEWCURSOR & 0.9491 & 48 & 0.0188 & 0.882 & 0.511 \\
\hline TETHER'VIEWCURSOR'SUBJECT & 8.7015 & 432 & 0.0201 & & \\
\hline DISPLACEMENT & 18.0382 & 5 & 3.6076 & 83.535 & 0.0001 \\
\hline DISPLACEMENT'SUBJECT & 1.9434 & 45 & 0.0432 & & \\
\hline TETHER'DISPLACEMENT & 0.3924 & 20 & 0.0198 & 0.744 & 0.777 \\
\hline TETHER'DISPLACEMENT'SUBJECT & 4.7494 & 180 & 0.0264 & & \\
\hline VIEW'DISPLACEMENT & 0.2204 & 5 & 0.0441 & 1.591 & 0.182 \\
\hline VIEW'DISPLACEMENT'SUBJECT & 1.2473 & 45 & 0.0277 & & \\
\hline TETHER'VIEW-DISPLACEMENT & 0.5923 & 20 & 0.0296 & 1.326 & 0.168 \\
\hline TETHERVIEW'DISPLACEMENT'SUBJECT & 4.021 & 180 & 0.0223 & & \\
\hline CURSOR'DISPLACEMENT & 1.7788 & 60 & 0.0297 & 1.364 & 0.046 \\
\hline CURSOR'DISPLACEMENT'SUBJECT & 11.832 & 540 & 0.0219 & & \\
\hline TETHER'CURSOR'DISPLACEMENT & 5.028 & 240 & 0.0200 & 0.978 & 0.581 \\
\hline TETHER'CURSOR'DISPLACEMENT'SUBJECT & 46.2718 & 2160 & 0.0214 & & \\
\hline VIEWCURSOR'DISPLACEMENT & 1.3992 & 60 & 0.0233 & 1.088 & $\overline{0.313}$ \\
\hline VIEW'CURSOR'DISPLACEMENT'SUBJECT & 11.5908 & 540 & 0.0215 & & \\
\hline TETHER'VIEWCURSOR'DISPLACEMENT & 5.4921 & 240 & 0.0229 & 1.152 & 0.064 \\
\hline TETHER'VIEWCURSOR'DISPLACEMENT"SUBJECT & 42.9116 & 2160 & 0.0199 & & \\
\hline
\end{tabular}


NOTE: This test controls the type I comparisonwise error rate, not the experimentwise error rate

$$
\text { Alpha }=0.05 d f=9 \quad M S E=0.144804
$$

Number of Means 2

Critical Range .01878

Means with the same letter are not significantly different.

Duncan Grouping

Mean N VIEWPT

$\begin{array}{llll}\text { A } & 0.597034 & 4200 & \text { b } \\ \text { B } & 0.575240 & 4200 & \text { a }\end{array}$

Alpha $=0.05 \quad d f=45 \quad M S E=0.045214$

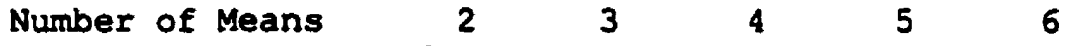

Critical Range $.01619 .01702 .01757 \quad 01797 \quad 01827$

Means with the same letter are not significantly different.

Duncan Grouping Mean N DISPLACEMENT

$\begin{array}{llll}\text { A } & 0.650492 & 1400 & -135 \\ \text { A } & & & \\ \text { A } & 0.643435 & 1400 & 135 \\ \text { B } & 0.587183 & 1400 & -90 \\ \text { B } & 0.575603 & 1400 & 90 \\ \text { B } & 0.536002 & 1400 & -45 \\ \text { C } & 0.524108 & 1400 & 45 \\ \text { C } & & & \end{array}$




\section{F-table for the Analysis of Variance (Dependent: Percent Overshoot)}

\begin{tabular}{|c|c|c|c|c|c|}
\hline Source & $\begin{array}{l}\text { sum of } \\
\text { Squares }\end{array}$ & of & $\begin{array}{l}\text { Doman } \\
\text { Squere }\end{array}$ & F-Value & $\frac{\text { P-Velve }}{0.0001}$ \\
\hline MEAN & 49.6602 & 1 & $\$ 9.6502$ & 233.783 & 0.0001 \\
\hline SUEJECT & 1.9114 & 8 & 0.2124 & & \\
\hline TETHER & 0.2868 & 4 & 0.0742 & 1.322 & 0.28 \\
\hline TETHER'SUEJECT & 2.02 & 36 & 0.0531 & & \\
\hline VIEW & 0.0048 & 1 & 0.0046 & 0.096 & 0.765 \\
\hline VIEW'SUBJECT & 0.4334 & 9 & 0.0462 & & \\
\hline TETHER VIEW & 0.2153 & 4 & 0.0538 & 4.593 & 0.004 \\
\hline TETHER'VIEW'SUBJECT & 0.4219 & 36) & 0.0117 & & \\
\hline CURSOR & 0.2374 & 12 & 0.0190 & 3.218 & 0.001 \\
\hline CURSOR 'SUBJECT & $0.68 \pi$ & 108 & 0.0061 & & \\
\hline TETHER'CURSOR & 0.301 & 48 & 0.0063 & 0.993 & 0.49 \\
\hline TETHER'CURSOR'SUBJECT & 2.728 & 432 & 0.0063 & & \\
\hline VIEWFCURSOR & 0.0784 & 12 & 0.0065 & 0.819 & 0.631 \\
\hline VIEW'CURSOR'SUBJECT & 0.8621 & 108 & 0.008 & & \\
\hline TETHER'VIEW'CURSOR & 0.4148 & 48 & 0.0086 & 1.38 & 0.053 \\
\hline TETHER'VIEWCURSOR'SUBNECT & 2.7034 & 432 & 0.0063 & & \\
\hline DISPLACEMENT & 8.1079 & 5 & $\mathbf{1 . 6 2 1 8}$ & 83.915 & 0.0001 \\
\hline DISPLACEMENT"SUBJECT & 0.8689 & ब45 & 0.0193 & & \\
\hline TETHER'DISPLACEMENT & 0.2128 & 20 & 0.0106 & 1.208 & 0.252 \\
\hline TETHER'DISPLACEMENT'SUBJECT & 1.5856 & 180 & 0.0088 & & \\
\hline VIEWDISPLACEMENT & 0.0343 & 5 & 0.0068 & 0.582 & 0.713 \\
\hline VIEW'DISPLACEMENT'SUBJECT & 0.5308 & 45 & 0.0118 & & \\
\hline TETHER'VIEW'DISPLACEMENT & 0.2415 & 20 & 0.0121 & 1.515 & 0.081 \\
\hline TETHER"VIEW"DISPLACEMENT"SUBJECT & 1.4346 & 180 & 0.008 & & \\
\hline CURSOR'DISPLACEMENT & 0.8868 & 60 & 0.0148 & 2.301 & 0.0001 \\
\hline CURSOR'DISPLACEMENT'SUBJECT & 3.4609 & 540 & 0.0034 & & \\
\hline TETHER'CURSOR'DISPLACEMENT & 1.8461 & 240 & 0.0077 & 1.084 & 0.192 \\
\hline TETHER"CURSOR'DISPLACEMENT'SUEJECT & 15.3285 & 2160 & 0.0071 & & \\
\hline VIEW'CURSOR'DISPLACEMENT & 0.355 & 60 & 0.0059 & 0.793 & 0.868 \\
\hline VIEW'CURSOR'DISPLACEMENT"SUBJECT & 4.0303 & 540 & 0.0075 & & \\
\hline TETHER'VIEW'CURSOR'DISPLACEMENT & 1.9239 & 240 & 0.008 & 1.074 & 0.219 \\
\hline TETHER'VIEW'CURSOR'DISPLACEMENT"SUBJECT & 16.1207 & 2160 & 0.0075 & & \\
\hline
\end{tabular}


NOTE: This test controls the type I comparisonwise error rate, not the experimentwise error rate

Alpha $=0.05$ df $=108$ MSE $=0.006236$

WARNING: Cell sizes are not equal.

Harmonic Mean of cell sizes= 624

\begin{tabular}{|c|c|c|c|c|c|c|}
\hline $\begin{array}{l}\text { Number of Means } \\
\text { Critical Range }\end{array}$ & $\begin{array}{r}2 \\
.00886\end{array}$ & $\begin{array}{r}3 \\
.00933\end{array}$ & $\begin{array}{r}4 \\
.00963\end{array}$ & $\begin{array}{r}5 \\
.00986\end{array}$ & $\begin{array}{r}6 \\
.01004\end{array}$ & $\begin{array}{r}7 \\
.01018\end{array}$ \\
\hline ritical Rane & $\begin{array}{r}8 \\
1030\end{array}$ & $\begin{array}{r}9 \\
01040^{9}\end{array}$ & $\begin{array}{r}10 \\
.01048\end{array}$ & $\begin{array}{r}11 \\
.01056\end{array}$ & $\begin{array}{r}12 \\
.01062\end{array}$ & .010 \\
\hline
\end{tabular}

Means with the same letter are not significantly different.

Duncan Grouping Mean N CURSOR

\begin{tabular}{|c|c|c|c|c|c|}
\hline & \multicolumn{2}{|l|}{$\begin{array}{l}\mathbf{A} \\
\mathbf{A}\end{array}$} & 0.088311 & 600 & 180 \\
\hline B & \multicolumn{2}{|l|}{ A } & 0.087334 & 600 & -180 \\
\hline B & \multicolumn{2}{|l|}{ A } & & & \\
\hline B & \multicolumn{2}{|l|}{ A } & 0.086289 & 600 & -160 \\
\hline B & \multicolumn{2}{|l|}{ A } & & & \\
\hline B & A & C & 0.082079 & 600 & -135 \\
\hline B & \multicolumn{2}{|l|}{ A } & & & \\
\hline B & \multirow{2}{*}{$\begin{array}{l}\mathrm{A} \\
\mathrm{A}\end{array}$} & \multirow{2}{*}{$c$} & 0.081715 & 600 & -120 \\
\hline B & & & & & \\
\hline B & \multirow{2}{*}{$\begin{array}{l}\text { A } \\
\text { A }\end{array}$} & C & 0.080259 & 600 & 160 \\
\hline B & & C & & & \\
\hline B & A & c & 0.080257 & 600 & 120 \\
\hline B & A & C & & & \\
\hline B & \multirow[t]{2}{*}{ A } & C & \multirow[t]{2}{*}{0.079677} & \multirow[t]{2}{*}{1200} & \multirow[t]{2}{*}{0} \\
\hline B & & C & & & \\
\hline B & D & C & \multirow{2}{*}{0.077753} & \multirow[t]{2}{*}{600} & \multirow[t]{2}{*}{-45} \\
\hline B & $D$ & C & & & \\
\hline B & D & C & \multirow[t]{2}{*}{0.077192} & \multirow[t]{2}{*}{600} & \multirow[t]{2}{*}{90} \\
\hline & \multirow{2}{*}{ D } & C & & & \\
\hline & & C & \multirow[t]{2}{*}{0.075543} & \multirow[t]{2}{*}{600} & \multirow[t]{2}{*}{135} \\
\hline & $D$ & C & & & \\
\hline & D & c & 0.072481 & 600 & -90 \\
\hline & \multicolumn{2}{|l|}{ D } & & & \\
\hline & \multicolumn{2}{|l|}{ D } & 0.068296 & 600 & 45 \\
\hline
\end{tabular}




$$
\text { Alpha }=0.05 \text { df }=45 \text { MSE }=0.020254
$$

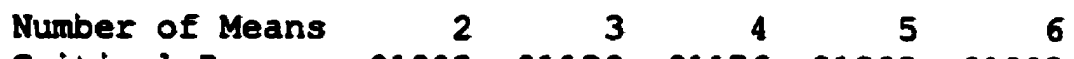

Critical Range $.01083 .01139 .01176 \quad 01203 \quad 01223$

Means with the same letter are not significantly different.

$\begin{array}{rrrrr}\text { Duncan Grouping } & \text { Mean } & \text { N } & \text { DISPIAC } \\ & \text { A } & 0.131606 & 1400 & -45 \\ & \text { B } & 0.116323 & 1400 & 45 \\ & \text { C } & 0.066306 & 1400 & -90 \\ & \text { C } & 0.061446 & 1400 & 90 \\ & \text { C } & 0.055768 & 1400 & -135 \\ \text { D } & \text { C } & 0.047207 & 1400 & 135 \\ \text { D } & & & & \end{array}$




\begin{tabular}{|c|c|c|c|c|c|}
\hline $\begin{array}{l}\text { F-table for the Analysis o } \\
\text { Souree }\end{array}$ & $\begin{array}{l}\text { (Dependel } \\
\text { sum of } \\
\frac{\text { squares }}{2805.05}\end{array}$ & of & $\begin{array}{l}\text { tion TIm } \\
\text { Moan } \\
\text { Square } \\
2905.051\end{array}$ & $\frac{F \cdot \text { Volue }}{1597.36}$ & $\frac{\text { P.Value }}{0.0001}$ \\
\hline $\begin{array}{l}\text { MEAN } \\
\text { SUEJECT }\end{array}$ & $\begin{array}{r}2805.05 \\
16.368 \\
\end{array}$ & $\frac{1}{9}$ & \begin{tabular}{r|}
2805.03 \\
18187
\end{tabular} & & 0.0001 \\
\hline TETHER & 13.7237 & 4 & 3.4300 & $18.3 \times 3$ & 0.0001 \\
\hline TETHER'SUBJECT & 6.7330 & 38 & 0.187 & & \\
\hline VEW & 0.0834 & 1 & 0.0534 & 0.624 & 0.46 \\
\hline VIEWSUEIECT & 1.3880 & 9 & 0.1543 & & \\
\hline TETHER' VIEW & 0.1657 & 4 & 0.0414 & 1.255 & 0.303 \\
\hline TETHER'VIEW'SUBJECT & 1.1883 & 38 & 0.033 & & \\
\hline CURSOR & 0.4118 & 12 & 0.0343 & 3.491 & 0.0001 \\
\hline CURSOR 'SUENECT & 1.0818 & 108 & 0.0098 & & \\
\hline TETHER'CURSOR & 0.6481 & 48 & 0.0135 & 1.075 & 0.346 \\
\hline TETHER'CURSOR'SUBJECT & 5.425 & 432 & 0.0128 & & \\
\hline VIEW'CURSOR & 0.0844 & 12 & 0.007 & 0.878 & 0.573 \\
\hline VIEW'CURSOR'SUBJECT & 0.8687 & 108 & 0.008 & & \\
\hline TETHER'VIEW'CURSOR & 0.6800 & 48 & 0.0142 & 1.364 & 0.064 \\
\hline TETHER'VIEW'CURSOR'SUBJECT & 4.5267 & 432 & 0.0105 & & \\
\hline DISPLACEMENT & 1.227 & 5 & 0.2454 & 9.339 & 0.0001 \\
\hline DISPLACEMENT"SUBJECT & 1.1824 & 46 & 0.0263 & & \\
\hline TETHER'DISPLACEMENT & 0.1786 & 20 & 0.0080 & 0.807 & 0.703 \\
\hline TETHER"DISPLACEMENT"SUBJECT & 1.9809 & 180 & 0.0111 & & \\
\hline VIEW'DISPLACEMENT & 0.1167 & 5 & 0.0233 & 1.678 & 0.159 \\
\hline VIEW'DISPLACEMENT"SUBSECT & 0.6258 & 45 & 0.0139 & & \\
\hline TETHER'VIEW'DISPLACEMENT & 0.1581 & 20 & 0.0079 & 0.790 & 0.712 \\
\hline TETHER'VIEW'DISPLACEMENT"SUBJECT & 1.7796 & 180 & 0.009 & & \\
\hline CURSOR"DISPLACEMENT & 1.1517 & 60 & 0.0192 & 2.137 & 0.0001 \\
\hline CURSOR'DISPLACEMENT"SUBSECT & 4.8508 & 540 & 0.009 & & \\
\hline TETHER'CURSOR'OISPLACEMENT & 2.6637 & 240 & 0.0111 & 0.998 & 0.498 \\
\hline TETHER"CURSOR"DISPLACEMENT"SUBJECT & 24.0185 & 2160 & 0.0111 & & \\
\hline VIEW'CURSOR'DISPLACEMENT & 0.6462 & 60 & 0.0100 & 1.088 & 0.346 \\
\hline VIEW"CURSOR"DISPLACEMENT"SUBJECT & 5.4441 & 340 & 0.0101 & & \\
\hline TETHER'VIEW'CURSOR'DISPLACEMENT & 2.5931 & 240 & 0.0108 & 1.004 & 0.475 \\
\hline TETHER'VIEW'CURSOR'OISPLACEMENT SUBJECT & 23.2528 & 2160 & 0.0108 & & \\
\hline
\end{tabular}



(Dependent: Reaction Time)

\begin{tabular}{|c|c|c|c|c|c|}
\hline Source & $\begin{array}{l}\text { Sum of } \\
\text { Squarue } \\
751 \text {. }\end{array}$ & df & $\frac{e^{2}}{751.429}$ & $\frac{F-\text { Value }}{881584}$ & $\frac{\text { P-Value }}{0.0001}$ \\
\hline SUBJECT & 7.6714 & $\overline{9}$ & 0.8524 & & \\
\hline VIEW & 0.226 & 1 & 0.226 & 3.627 & 0.089 \\
\hline VIEW-SUBJECT & 0.5607 & $\overline{9}$ & 0.0623 & & \\
\hline CURSOR & 0.4381 & 12 & 0.0365 & 1.688 & 0.084 \\
\hline CURSOR 'SUBJECT & 2.366 & 108 & 0.0219 & & \\
\hline VIEW'CURSOR & 0.1846 & 12 & 0.0154 & 1.497 & 0.136 \\
\hline VIEW'CURSOR'SUBJECT & 1.1098 & 108 & 0.0103 & & \\
\hline DISPLACEMENT & 0.3864 & $\sqrt{5}$ & 0.0773 & 5.554 & 0.0001 \\
\hline DISPLACEMENT'SUBJECT & 0.6261 & 45 & 0.0139 & & \\
\hline VIEW'DISPLACEMENT & 0.0272 & 5 & 0.0054 & 0.552 & 0.736 \\
\hline VIEW'DISPLACEMENT"SUBJECT & 0.4443 & 45 & 0.0099 & & \\
\hline CURSOR'DISPLACEMENT & 0.9957 & 60 & 0.0166 & 1.291 & 0.078 \\
\hline CURSOR'DISPLACEMENT'SUBJECT & 6.9439 & 540 & 0.0129 & & \\
\hline VIEW'CURSOR'DISPLACEMENT & 0.8043 & 60 & 0.0134 & 1.137 & 0.233 \\
\hline VIEW'CURSOR'DISPLACEMENT"SUBJECT & 6.3665 & 540 & 0.0118 & & \\
\hline
\end{tabular}


NOTE: This test controls the type I comparisonwise error rate, not the experimentwise error rate

$$
\text { Alpha }=0.05 \quad d f=36 \quad M S E=0.200842
$$

Number of Means $\quad 2 \quad 3 \quad 4 \quad 5$

Critical Range $.03136 .03297 \quad 03402.03477$

Means with the same letter are not significantly different.

$$
\text { Duncan Grouping Mean N TETHER }
$$

$\begin{array}{llll}\text { A } & 0.69330 & 1680 & \text { w00 } \\ \text { B } & 0.59290 & 1680 & \text { w01 } \\ \text { B } & 0.58990 & 1680 & \text { w99 } \\ \text { B } & 0.58815 & 1680 & \text { w02 } \\ \text { B } & 0.58579 & 1680 & \text { w03 } \\ \text { B } & & & \end{array}$

$$
\text { Alpha }=0.05 \quad d f=45 \quad \text { MSE }=0.027557
$$

$\begin{array}{lrrrrr}\text { Number of Means } & 2 & 3 & 4 & 5 & 6 \\ \text { Critical Range } & .01264 & .01329 & .01372 & .01403 & .01426\end{array}$

Means with the same letter are not significantly different.

Duncan Grouping Mean N DISPLACEMENT

$\begin{array}{llll}\text { A } & 0.629257 & 1400 & 135 \\ \text { A } & & & \\ \text { A } & 0.618200 & 1400 & -135 \\ \text { A } & 0.617900 & 1400 & 45 \\ \text { A } & 0.604786 & 1400 & 90 \\ \text { B } & & & \\ \text { B } & 0.596629 & 1400 & -90 \\ \text { B } & 0.593286 & 1400 & -45 \\ \text { B } & & & \end{array}$


Alpha $=0.05$ df $=108$ MSE $=0.01005$

WARNING: Cell sizes are not equal.

Harmonic Mean of cell sizes= 624

$\begin{array}{lrrrrrr}\text { Number of Means } & 2 & 3 & 4 & 5 & 6 & 7 \\ \text { Critical Range } & .01125 & .01184 & .01223 & .01252 & .01274 & .01292\end{array}$

$\begin{array}{lllllll}\text { Number of Means } & 8 & 9 & 10 & 11 & 12 & 13\end{array}$

Critical Range $.01307 .01320 .01331 .01340 .01348 \quad .01356$

Means with the same letter are not significantly different.

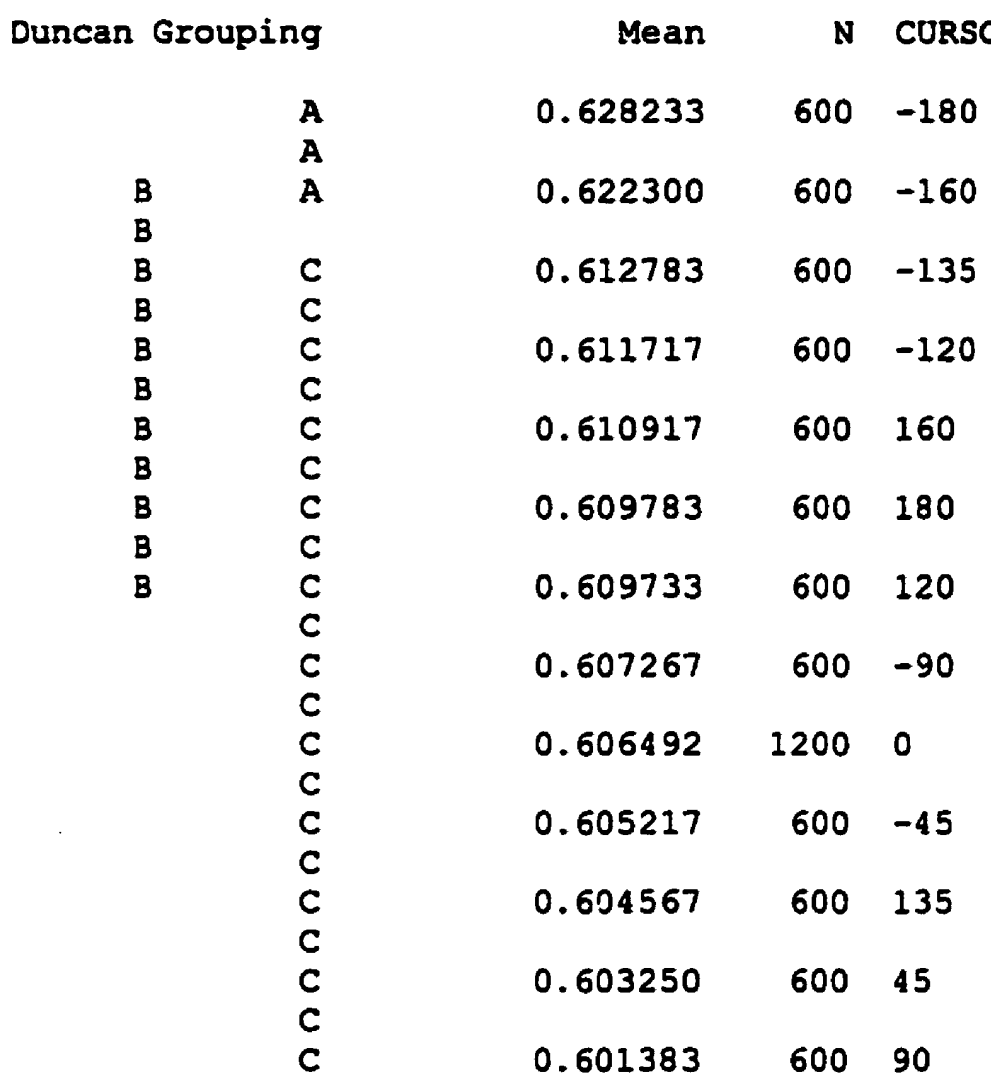


F-table for the Analysis of Variance (Dependent: Frequency of Control Reversals)

\begin{tabular}{|c|c|c|c|c|c|}
\hline Source & $\begin{array}{l}\text { sum of } \\
\text { squeres }\end{array}$ & df & Square & F-Value & $\frac{\text { P.value }}{0.001}$ \\
\hline SUBJECT & 18.0774 & 9 & $\frac{44.0261}{2.0086}$ & 21.918 & \\
\hline TETHER & 15.2832 & 4 & 3.8208 & 12.889 & 0.000 \\
\hline TETHER'SUBJECT & 10.6719 & 36 & 0.2964 & & \\
\hline VIEW & 0.0104 & 1 & 0.0104 & 0.167 & $\overline{0.692}$ \\
\hline VIEW & 0.5801 & 9 & 0.0622 & & \\
\hline TETHER'VIEW & 0.3588 & 4 & 0.0897 & 0.8 & 0.47 \\
\hline TETHERVIEW'SUBJECT & 3.5899 & 36 & 0.0997 & & \\
\hline CURSOR & 2.1915 & 12 & 0.1826 & 2.516 & 0.00 \\
\hline CURSOR "SUBJECT & 7.8392 & 108 & 0.0726 & & \\
\hline TETHER'CURSOR & 9.4418 & 48 & 0.1967 & 2.908 & 0.000 \\
\hline TETHER'CURSOR'SUBJECT & 29.2197 & 432 & 0.0676 & & \\
\hline VIEWCCURSOR & 0.5446 & 12 & 0.0454 & 0.728 & 0.722 \\
\hline VIEW'CURSOR'SUBJECT & 6.7349 & 108 & 0.0624 & & \\
\hline TETHER'VIEWCURSOR & 3.2528 & 48 & 0.0678 & 1.147 & 0.24 \\
\hline TETHER'VEW'CURSOR'SUBJECT & 25.5318 & 432 & 0.0591 & & \\
\hline DISPLACEMENT & 1.266 & 5 & 0.2532 & 1.29 & 0.285 \\
\hline DISPLACEMENT SUBJECT & 8.8314 & 45 & 0.1963 & & \\
\hline TETHER'DISPLACEMENT & 0.7776 & 20 & 0.0389 & 0.659 & 0.868 \\
\hline TETHER'DISPLACEMENT'SUBJECT & 10.625 & 180 & 0.059 & & \\
\hline VIEW'DISPLACEMENT & 0.1723 & 5 & 0.0345 & 0.419 & 0.83 \\
\hline VIEW'DISPLACEMENT'SUBJECT & 3.7033 & 45 & 0.0823 & & \\
\hline TETHER'VIEW'DISPLACEMENT & 1.2873 & 20 & 0.0644 & 1.092 & 0.361 \\
\hline TETHER'VIEW'DISPLACEMENT"SUBJECT & 10.6063 & 180 & 0.0589 & & \\
\hline CURSOR"DISPLACEMENT & 3.1623 & 60 & 0.0527 & 0.898 & 0.692 \\
\hline CURSOR'DISPLACEMENT'SUBJECT & 31.7069 & 540 & 0.0587 & & \\
\hline TETHER"CURSOR'DISPLACEMENT & 17.1274 & 240 & 0.0714 & 1.233 & $\overline{0.012}$ \\
\hline TETHER"CURSOR'DISPLACEMENT"SUBJECT & 125.003 & 2160 & 0.0579 & & \\
\hline VIEW'CURSOR'DISPLACEMENT & 4.3277 & 60 & 0.0721 & 1.271 & 0.091 \\
\hline VEW'CURSOR"DISPLACEMENT"SUBJECT & 30.6467 & 540 & 0.0568 & & \\
\hline TETHER'VIEW'CURSOR'DISPLACEMENT & 13.321 & 240 & 0.0555 & 0.929 & $0.76 \mathrm{~s}$ \\
\hline TETHER'VIEW'CURSOR"DISPLACEMENT"SUBJECT & 129.102 & 2160 & 0.0598 & & \\
\hline
\end{tabular}


Duncan's Multiple Range Test for variable: Frequency of Control Reversals

NOTE: This test controls the type I comparisonwise error rate, not the experimentwise error rate

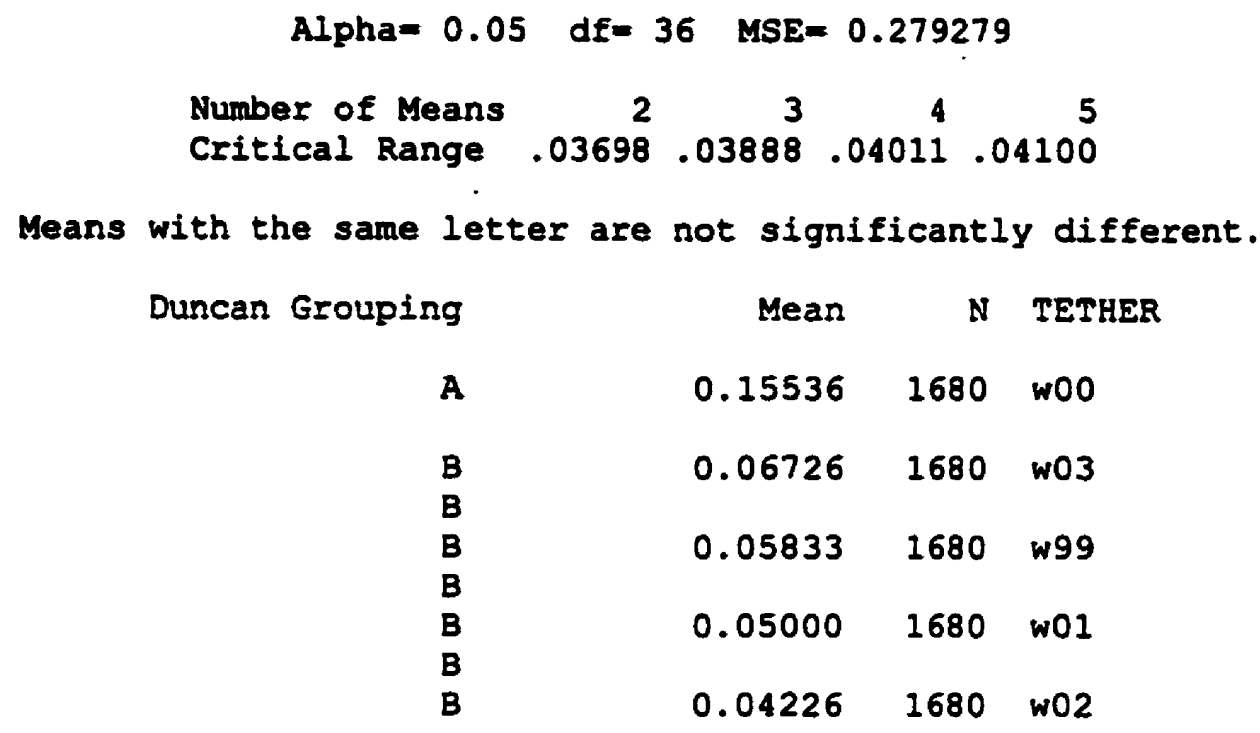


Alpha $=0.05 \quad d f=108 \quad$ MSE $=0.073925$

WARNING: Cell sizes are not equal.

Harmonic Mean of cell sizes= 624

$\begin{array}{lrrrrrr}\text { Number of Means } & 2 & 3 & 4 & 5 & 6 & 7 \\ \text { Critical Range } & .03051 & .03211 & .03317 & .03395 & .03456 & .03504 \\ \begin{array}{l}\text { Number of Means } \\ \text { Critical Range }\end{array} & .03545 & .03579 & .03609 & .03635 & .03657 & .03677\end{array}$

Means with the same letter are not significantly different.

\begin{tabular}{|c|c|c|c|c|c|c|}
\hline Duncan $\mathrm{G}$ & oug & & & Mean & $\mathrm{N}$ & CURSO \\
\hline & & $\begin{array}{l}\text { A } \\
\text { A }\end{array}$ & & 0.11000 & 600 & -180 \\
\hline B & & A & & 0.10667 & 600 & 180 \\
\hline B & & A & & & & \\
\hline B & & A & C & 0.08167 & 600 & 160 \\
\hline B & & A & C & & & \\
\hline B & D & A & C & 0.07667 & 600 & 120 \\
\hline B & D & A & c & & & \\
\hline B & D & A & C & 0.07667 & 600 & -45 \\
\hline B & D & & C & & & \\
\hline B & D & & C & 0.07333 & 600 & -90 \\
\hline B & D & & C & & & \\
\hline B & D & & C & 0.07167 & 600 & 90 \\
\hline B & D & & C & & & \\
\hline B & D & & C & 0.07167 & 600 & -135 \\
\hline & D & & C & & & \\
\hline & D & & C & 0.07000 & 600 & -120 \\
\hline & D & & C & & & \\
\hline & D & & C & 0.06833 & 1200 & 0 \\
\hline & D & & C & & & \\
\hline & D & & C & 0.06500 & 600 & -160 \\
\hline & D & & C & & & \\
\hline & D & & C & 0.06167 & 600 & 135 \\
\hline & D & & & & & \\
\hline & D & & & 0.04333 & 600 & 45 \\
\hline
\end{tabular}


B2 - Tracking Experiments

F-table for the Analysis of Variance for the Planar View (Dependent: RMS error)

\begin{tabular}{|c|c|c|c|c|c|}
\hline souree & squares & df & Squane & F-Value & P-Value \\
\hline MEAN & 1168354 & 1 & 1168354 & 644.685 & 0.0001 \\
\hline SUBJECT & 16310.8 & $\overline{9}$ & 1812.29 & & \\
\hline TETHER & 180.78 & 4 & 45.1949 & 0.298 & 0.877 \\
\hline TETHER'SUBJECT & 5460.59 & 36 & 151.683 & & \\
\hline PHASE & 300.404 & 9 & 33.3782 & 2.55 & 0.012 \\
\hline PHASE'SUBJECT & 1060.12 & 81 & 13.0879 & & \\
\hline TETHER'PHASE & 532.571 & 36 & 14.7936 & 1.19 & 0.218 \\
\hline TETHER'PHASE'SUBJECT & 4029.11 & $\overline{324}$ & 12.4355 & & \\
\hline
\end{tabular}

F-table for the Analysis of Variance for the Planar View without Subject 9's Data (Dependent: RMS error)

\begin{tabular}{|c|c|c|c|c|c|}
\hline Source & Squares & dif & & F-Value & P.Value \\
\hline MEAN & 990215 & 1 & 990215 & 1114.91 & 0.0001 \\
\hline SUBJECT & 7105.25 & 8 & 888.156 & & \\
\hline TETHER & 282.346 & $\overline{4}$ & 70.5864 & 0.498 & 0.737 \\
\hline TETHER`SUBJECT & 4535.72 & 32 & 141.741 & & \\
\hline PHASE & 293.725 & $\overline{9}$ & 32.6361 & 2.911 & 0.005 \\
\hline PHASESUBJECT & 807.296 & $\overline{72}$ & 11.2124 & & \\
\hline TETHER'PHASE & 544.17 & $\overline{36}$ & 15.1158 & 1.541 & 0.029 \\
\hline TETHER'PHASE`SUBJECT & 2824.26 & 288 & 9.8064 & & \\
\hline
\end{tabular}


F-table for the Analysis of Variance for the Perspective View without Tether w02 Data (Dependent: RMS error)

\begin{tabular}{|c|c|c|c|c|c|}
\hline Source & & df & & F-Value & P.Value \\
\hline MEAN & 924459 & 1 & 924459 & 704.579 & 0.0001 \\
\hline SUBJECT & 11808.7 & 9 & 1312.07 & & \\
\hline TETHER & $\overline{158.521}$ & 3 & 52.8404 & 0.384 & 0.765 \\
\hline TETHER'SUBJECT & 3716.48 & 27 & 137.647 & & \\
\hline PHASE & 209.023 & 9 & 23.2247 & 1.671 & 0.11 \\
\hline PHASE'SUBJECT & 1126.07 & 81 & 13.9021 & & \\
\hline TETHER'PHASE & 552.495 & 27 & 20.4628 & 1.599 & 0.035 \\
\hline TETHER'PHASE'SUBJECT & 3110.59 & 243 & 12.8008 & & \\
\hline
\end{tabular}




\section{Questionnaire}

For participation in the experiment "Dynamic Tethering for Enhanced Remote Control and Navigation"

Subject No.:

Date:

Time:

1. Gender (please circle one):

$\mathbf{M}$

$\mathbf{F}$

2. Age (please circle one):

$<20 \quad 20-30 \quad 30-40$

$40-50 \quad 50-60>60$

3. Do you ordinarily wear corrective lenses of any kind?

Yes

No

If yes, are you wearing prescribed lenses right now?

Yes

No

4. Are you left or right handed?

Left

Right

Which hand do you prefer to use in a manual task, i.e. using a mouse?

Left

Right

5. How many years of post-secondary education have you completed: (Please check one) none $1-4$ years $>4$ years

6. Please indicate your occupation: (Please check one and specify if necessary)

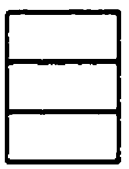

Student Engineer Researcher

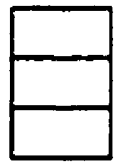

Pilot

Heavy machine operator

Other:

7. Have you ever participated in a target tracking tests or activities prior to this experiment?

Yes

No

If yes, please describe and indicate hours involved below:

Hours (please circle one):

$<5$ hours

5-50 hours

$>50$ hours

Page 1 of 2 - Stop! Please answer questions on the next page after the experiment. 
Post-experiment questions:

You are about to be presented with the five displays you have used in this experiment. After each presentation rank each by placing a mark in an appropriate position on the three scales below. Label your response (1) for the first display, (2) for the second display, etc...

\section{Understandability:}

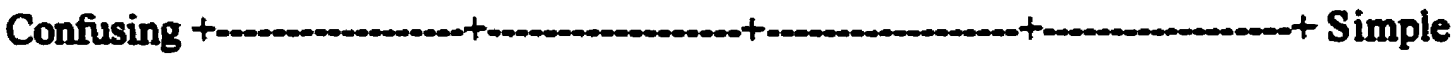

Ease of Use:

Difficult to + use use

Response to on-screen motion:

Disorienting +...- No significant reaction

This section is to be filled in by the experimenter.

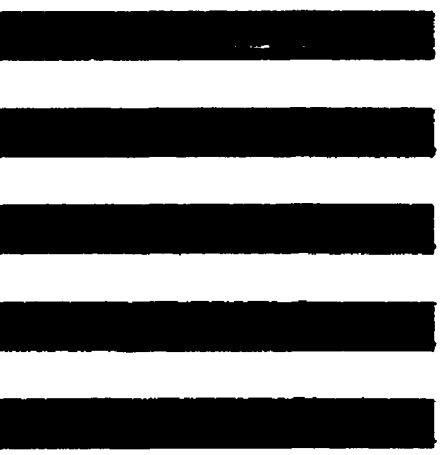




\section{Appendix D - Spreadsheet: Calculation of Forcing Function Frequencies}

As discussed in Section 8.1.2, the eleven track frequencies were divided evenly amongst the chosen tether frequencies. The natural frequency settings were distributed as follows:

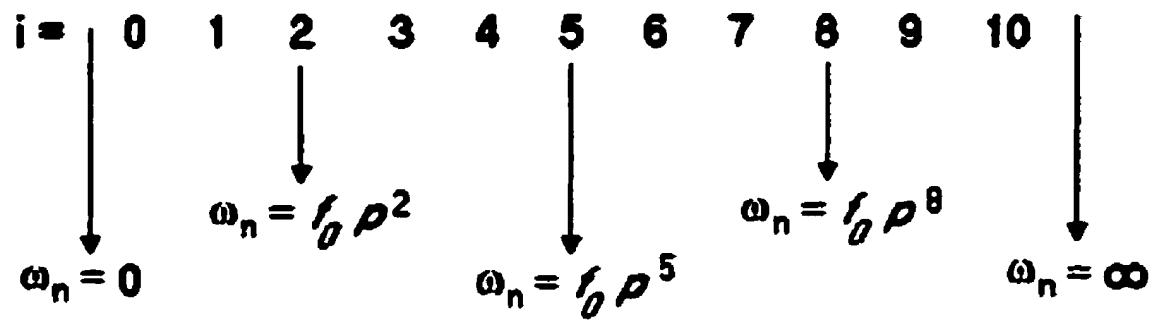

The calculations of each tether value is based on the approximate eye-to-screen distance and the approximate image radius on the screen as illustrated in the following diagram:

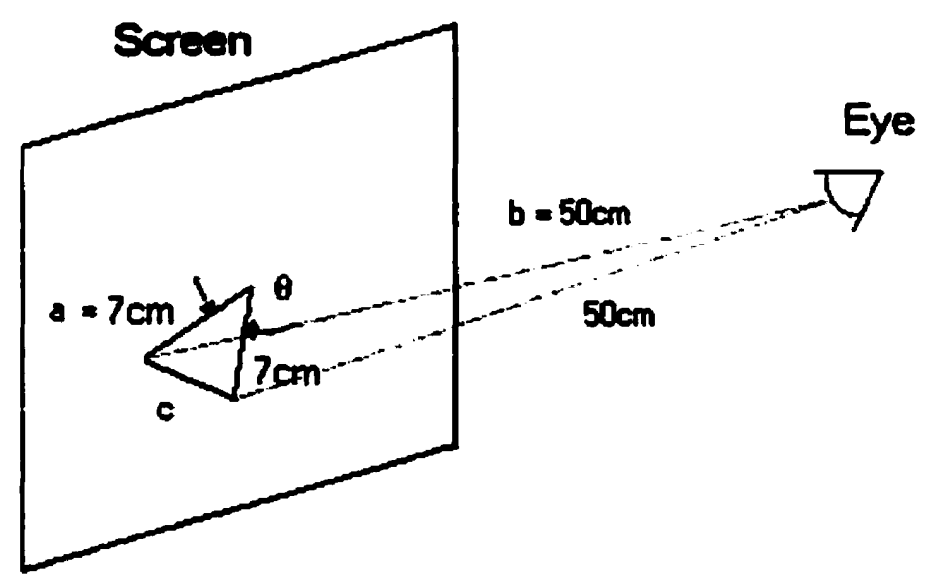

The minimum distance (C) that the end of the cursor must trace in one second in order to produce a minimal perceptible retinal image motion ( $\gamma$ per second) is as follows:

By the cosine law,

$c^{2}=a^{2}+a^{2}-2(a)(a) \cos (\theta)$

and

$$
c^{2}=b^{2}+b^{2}-2(b)(b) \cos (\gamma)
$$


where

\begin{tabular}{|c|c|}
\hline $25-50 \mathrm{sec} \operatorname{arc} / \sec$ (< $1 \mathrm{~min}$ ard/second) & $\begin{array}{l}\text { Minimum threshold for real motion perception } \\
\text { (oceurs at } i=0 \text { ) }\end{array}$ \\
\hline $60-120 \sec$ ardsec $(1-2 \mathrm{~min}$ are/second) & $\begin{array}{l}\text { Minimum threshold for induced motion perception } \\
\text { (oceurs at } i=2 \rightarrow \text { wol) }\end{array}$ \\
\hline $10-50 \mathrm{~min}$ ard $/ \mathrm{sec}$ & $\begin{array}{l}\text { Maximum threshold for induced motion perception } \\
\text { (occurs between } i=5 \rightarrow \text { w02 and } i=8 \rightarrow \text { w03) }\end{array}$ \\
\hline$>20 \mathrm{~Hz}$ & $\begin{array}{l}\text { Real motion perception becomes a blur (tracking at } \\
\text { frequencies greater than } 1 \mathrm{~Hz} \text { becomes near } \\
\text { impossible, c.f. Section 5.1.3) }\end{array}$ \\
\hline
\end{tabular}

and

$\theta(t)=\sum_{i=0}^{10} B q^{-i} \sin \left(2 \pi f_{0} p^{\prime} t+\phi(i)\right)$

$\mathrm{B}(\mathrm{i}=2)=\mathrm{Bq}^{-2}=\pi / 2$ and $\mathrm{B}(\mathrm{i}=8)=\mathrm{Bq}^{-8}=\pi / 6$, where determined by trail and error to produce a reasonable tracking task.

A spreadsheet calculation of all remaining frequencies and their associated amplitudes is included on the next page. 


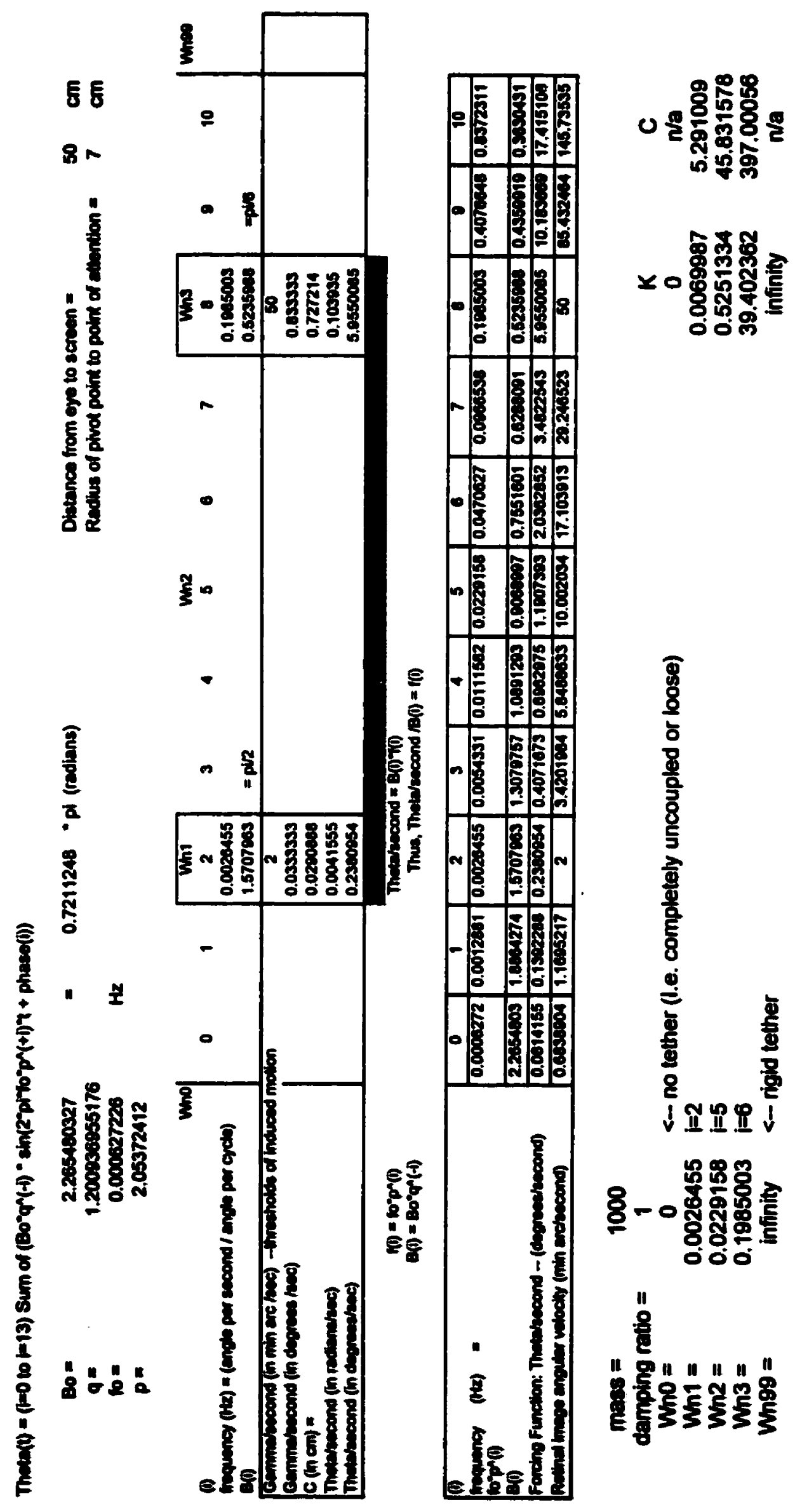




$$
\underset{m_{1} \ddot{x}_{1}}{\longrightarrow} \underset{\sum F=m a}{L\left(\dot{x}_{2}-\dot{x}_{1}\right)}
$$

Following endpoint $x_{2}$ gives:

$$
F \ddot{x}_{1}+c\left(\dot{x}_{2}-\dot{x}_{1}\right)+k\left(x_{2}-x_{1}\right)=m_{1} \ddot{x}_{1}
$$

$\because$ there are no external forces acting on

the view other than the spring-damper system

$$
\therefore \quad F \ddot{x}_{1}=0
$$

$$
\begin{aligned}
& c \dot{x}_{2}-c \dot{x}_{1}+k x_{2}-k x_{1}=m_{1} \ddot{x}_{1} \\
& m_{1} \ddot{x}_{1}+c \dot{x}_{1}+k x_{1}=\underbrace{c \dot{x}_{2}+k x_{3}}_{\text {Driving Force / Cursor Control }}
\end{aligned}
$$

$$
\begin{aligned}
& m_{1} \ddot{x}_{1}+c \dot{x}_{1}+k x_{1}=f\left(x_{2}, \dot{x}_{2}\right) \text { : } \\
& f\left(x_{2}, \dot{x}_{2}\right)=c \dot{x}_{2}+k x_{2} \text { and is known } \\
& x_{2} \text { is the displacement of the view given } \\
& \text { that the tether is rigid 0l object displacement) } \\
& \dot{x}_{2} \text { is the velocity of the } \\
& \text { controlled object }
\end{aligned}
$$

Using first order estimations:

$$
\begin{aligned}
& m_{1} \ddot{x}_{1}+c \dot{x}_{1}+k x_{1}-f\left(x_{2}, \dot{x}_{2}\right) \\
& \quad m_{1}\left(\frac{x_{t-1}-2 x_{t}+x_{t-1}}{\Delta t^{2}}\right)_{1}+c\left(\frac{x_{t-1}-x_{t}}{\Delta t}\right)_{-1}+k\left(x_{t}\right)=f\left(x_{2}, \dot{x}_{2}\right)
\end{aligned}
$$




$$
\begin{aligned}
& m_{1}\left(\frac{x_{t-1}-x_{t}+x_{t-1}}{\Delta t^{2}}\right)_{-1}+\frac{c}{2}\left(\frac{2 x_{t-1}-2 x_{t}}{\Delta t}\right)_{1}+k\left(x_{1}\right)_{1}=f\left(x_{2}, \dot{x}_{2}\right)+\frac{m_{1} x_{l}}{\Delta t^{2}} \\
& m_{1}\left(\frac{x_{t-1}-x_{t}+x_{t-1}}{\Delta t^{2}}\right)_{-1}+\frac{c}{2}\left(\frac{x_{t-1}-x_{t}}{\Delta l}\right)_{-1}+k\left(x_{t}\right)=f\left(x_{2} \cdot \dot{x}_{2}\right)+\frac{m_{1} x_{t}}{\Delta t^{2}}-\frac{c}{2} \dot{x}_{t} \\
& m_{1}\left(\frac{x_{t 01}}{\Delta t^{2}}-\frac{\dot{x}_{l}}{\Delta t}\right)+\frac{c}{2}\left(\frac{x_{t 01}}{\Delta t}-\frac{x_{t}}{\Delta l}\right)+k\left(x_{1}\right)=f\left(x_{2}, \dot{x}_{2}\right)+\frac{m_{1} x_{t}}{\Delta t^{2}}-\frac{c}{2} \dot{x}_{t} \\
& k\left(x_{1}\right)+m_{1} \frac{x_{t-1}}{\Delta t^{2}}+\frac{c}{2} \frac{x_{t-1}}{\Delta t}=f\left(x_{2} \cdot \dot{x}_{2}\right)+\left(\frac{m_{1}}{\Delta t^{2}}-\frac{c}{2 \Delta t}\right) x_{t}+\left(\frac{m_{1}}{\Delta t}-\frac{c}{2}\right) \dot{x}_{t} \\
& \left(\frac{m_{1}}{\Delta t^{2}}+\frac{c}{2 \Delta t}\right) x_{t-1}=f\left(x_{2}, \dot{x}_{2}\right)+\left(\frac{m_{1}}{\Delta t^{2}}-\frac{c}{2 \Delta t}-k\right) x_{t}+\left(\frac{m_{1}}{\Delta t}-\frac{c}{2}\right) \dot{x}_{t}
\end{aligned}
$$

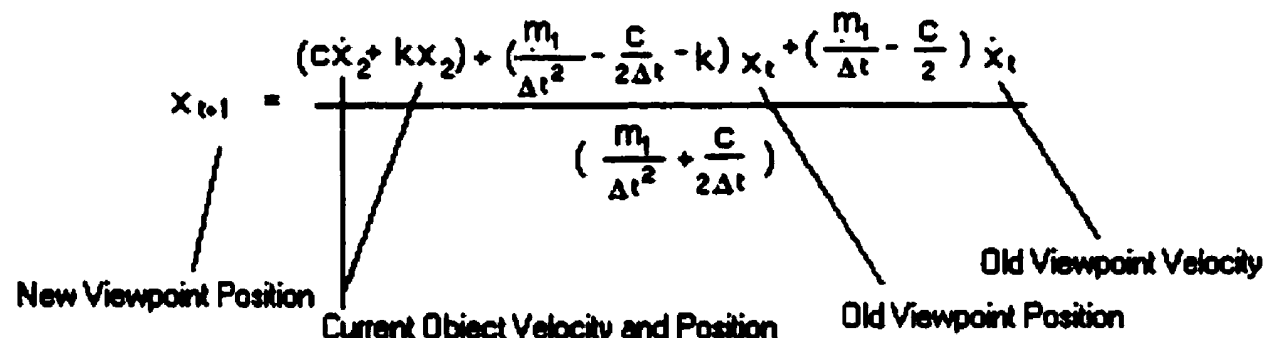




\section{References}

Aretz, A.J., (1991), "The Design of Electronic Map Displays". Human Factors, 33(1), 85-101.

Barfield W. and Salvendy, G. (1985). "Perceptual and cognitive aspects of threedimensional rotations for computer aided design (CAD) systems". Proceedings of the Human Factors Society - 29 th Annual Meeting. Vol. 11. Baltimore, Maryland. September 29 - October 3, 1985.

Bauerschmidt, D.K., and Roscoe, S.N. (1960). "A comparative evaluation of a pursuit moving-airplane steering display.", IRE Transactions on Human Factors in Electronics,HFE-1(2), 62-66.

Beringer, D.B., Williges, R.C., and Roscoe, S.N. (1975), "The transition of experienced pilots to a frequency-separated aircraft attitude display. Human Factors, 17, 401-414.

Bermotat, R. K. (1970). "Rotation of visual reference system and its influence on control quality". IEEE Trans. on Man-Machine Systems, MMS - 11: 129-131.

Bennett, K.B., Magy, A.L., and Flach, J. (1997) Chapter 20 "Visual Displays", in Salvendy (Ed.) Handbook of Human Factors, $2^{\text {nd }}$ Edition. John Wiley \& Sons, Inc. 0 1997, New York.

Berthoz, A. (1991). Chapter 6: "Reference frames for the perception and control of movement" in J. Paillard's (Ed.) Brain and Space. Oxford University Press $\odot$ 1991, Oxford, pp. 81-111.

Burns, C.M. (1998) PhD Dissertation: "The affects of spatial and temporal proximity of means-end information in ecological display design for an industrial simulation". University of Toronto, Department of Mechanical and Industrial Engineering.

Boff, Kaufman, and Thomas (Ed's), (1986). Handbook of Human Perception and Performance, Wiley, 1986, O 1986, John Wiley \& Sons, Inc.

Boff, K.R., and Lincoln, J.E.(Ed's), (1988). Human Perception and Performance, Volume I. Engineering Data Compendium. Harry G. Armstrong Aerospace Medical Research Laboratory, Wright-Patterson Air Force Base, Ohio, 1988.

Browne, R.C. (1945) "Trial of two attitude indicators". Farnborough, Hampshire, England: Royal Aircraft Establishment, Flying Personnel Research Committee, Air Ministry Reports 611 and 611A, 1945.

Browne, R.C. (1954) "Figure and ground in a two-dimensional display." Journal of Applied Psychology, 1954, 38, 462-467.

Cooper, L.A., (1975), "Mental rotation of random two-dimensional shapes." Cognitive Psychology, 1975, 7, 20-43. 
Cooper, L. A., and Podgorny, P. (1976), "Mental transformations and visual comparison processes: Effects of complexity and Similarity". Journal of Experimental Psychology: Human Perception and Performance, 2-4, 503-514.

Cooper, L.A., and Shepard, R.N. (1973), "The time required to prepare for a rotated stimulus." Memory and Cognition, 1, 246-250.

Craig, K. M., Hartzell, J.E., and Dunbar, S.L. (1983), "Helicopter pilot response as a function of compatibility of the control-display configuration". $19^{\text {th }}$ Anmual Conference on Mamual Control, p.100.

Cunningham, H.A. and Pavel, M. (1990). "Rotational wind" indicator enhances control of rotated displays. In Proceedings of Engineering Foundation Conference on Teleoperators and Virtual Environments, Santa Barbara, CA, March.

Dorf, R.C., and Bishop, R.H. (1995). Modern Control Systems. () 1995 Addison-Wesley Publishing Company, Inc. Reading, USA.

Ellis, S.R., Tyler, M., Kim, W.S., and Stark, L., (1991). "Three-Dimensional Tracking with Misalignment Between Display and Control Axes". SAE Technical Paper Series, SAE International, The Engineering Society For Advancing Mobility Land Sea Air and Space. $21^{\text {st }}$ International Conference on Environmental Systems, San Francisco, California, July 15-18, 1991.

Knight J.L., Jr. (1987). Chapter 2.7 "Manual control and tracking", in Salvendy (Ed.) Handbook of Human Factors. O 1987, John Wiley \& Sons, Inc., 182-218.

Faye, E.L., Wickens, C.D., (1995). "Strategies for Display Integration in Navigational Guidance and Situation Awareness". Technical Report, April 1995. Aviation Research Laboratory Institute of Aviation, University of Illinois at UrbanaChampaign.

Finke, R.A., and Shepard, R.N., (1986). Chapter 37 "Visual functions of mental imagery", in Boff, Kaufiman, and Thomas (Ed.'s) Handbook of Perception and Human Performance. O 1986, John Wiley \& Sons, Inc., 37-1-55.

Fogel, Larry J., (1959). "A New Concept: The Kinalog System", Journal of the Human Factors Society, Vol. 1, No. 2, pages 30-37, April 1959.

Gavriel Salvendy (Ed.), (1997). Handbook of Human Factors and Ergonomics, $2^{\text {nd }}$ Edition. John Wiley and Sons, Inc., O1997. New York.

Gibbs, C. B. (1962) "Controller design: Interactions of controlling limbs, time-lags and gains in positional and velocity systems". Ergonomics. 5(2), 385-402.

Glumm, M.M., Marshak, W.P., Branscome, T.A., Wesler, M.Mc., Patton, D.J., Mullins, L.L., (1998). “A Comparison of Soldier Performance Using Current Land Navigation 
Equipment With Information Integrated on a Helmet-Mounted Display". Army Research Laboratory, April 1998. (ARL-TR-1604)

Graham, C.H. (1965). "Perception of movement" in C.H. Graham (Ed.), Vision and visual perception. New York: Wiley.

Holden, J.G., Flach, J.M., and Donchin, Y., (1999). "Perceptual-motor coordination in an endoscopic surgery simulation". Surgical Endoscopy: Ultrasound and Interventional Techniques, 1999, vol. 13.

Huggins and Getty (1982). "Display-Control Compatibility in 3-D displays, 2: Effects of Cue Symmetry". Technical Report, BBN Report No. 5101, N00014-80-C-0750, Engineering Psychology Programs, Office of Naval Research, Arlington, VA, USA. November 15, 1982.

Ince, F., Williges, R., and Roscoe, S., (1975). "Aircraft Simulator Motion and the Order of Merit of Flight Attitude and Steering Guidance Displays". Human Factors, 1975, 17(4), 388-400.

Johnson, S.L., and Roscoe, S.N. (1972). "What Moves, the airplane or the world?" Human Factors, 14, 107-129.

Leibowitz, H. (1955). "Effect of reference lines on the discrimination of movement". Journal of the Optical Society of America, 45, 829-830.

Liu, Y. (1997). Chapter 51 "Software User Interface Design", in Salvendy (Ed.) Handbook of Human Factors, $2^{\text {nd }}$ Edition. John Wiley \& Sons, Inc. 0 1997, New York.

Macedo, J.A., Kaber, D.B., Endsley, M.R., Powanusorn, P., and Myung, S., (1998). "The Effect of Automated Compensation for Incongruent Axes on Teleoperator Performance". Human Factors, 40(4), 541-553.

Macedo, J.A., Myung, S., and Kaber, D.B., (1997). "Automated Compensation for Incongruent Display-Controller Axes in Teleoperation". Proceedings ANS $7^{\text {th }}$ Topical Meeting on Robotics and Remote Systems.

Majchrzak, A., Chang, T., Barfield, W., Eberts, R., Salvendy, G., (1987). Human Aspects of Computer-Aided Design. Philadelphia, 01987 Taylor \& Francis.

Marshak, W.P., Kuperman, G., Ramsey, E.G., and Wilson, D. (1987). "Situational awareness in map displays". In Proceedings of the Human Factors Society $31^{s t}$ Annual Meeting (pp. 533-535). Santa Monica, CA: Human Factors Society.

McGovern, D., in Ellis, R. Stephen (Ed), (1991). Pictorial communication in virtual and real environments, $O$ 1991, Taylor \& Francis Ltd., New York. 
Milgram, P., and Colquhoun Jr., H.W. (1999a). "A Taxonomy of Real and Virtual World Display Integration". Chapter 1 in Y. Ohta and H. Tamura (Eds.), Mixed Reality: Merging Real and Virtual Worlds, Ohmasha, Ltd., O 1999, pp. 5-30.

Milgram, P., and Colquhoun, H. W., (1999b). "A Framework for Relating Head-Mounted Displays to Mixed Reality Displays", Proceedings of the Human Factors and

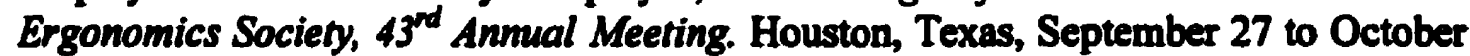
1, 1999, pp. 1177-1181.

Milgram, P, and Kishino, F., (1994). "A taxonomy of mixed reality visual displays", IEICE (Institute of Electronics, Information and Communication Engineers) Transactions on Information and Systems, Special issue on Networked Reality, Dec. 1994.

Olmos, O., Wickens, C.D., and Chudy, A., (1997). "Tactical Displays for Combat Awareness: An Examination of Dimensionality and Frame of Reference Concepts, and the Application of Cognitive Engineering". Proceedings of the $9^{\text {th }}$ International Symposium on Aviation Psychology. Columbus, OH: Dept. of Aerospace Engineering, Applied Mechanics and Aviation.

Paillard, J., (1991). Chapter 10: "Motor and representational framing of space" in J. Paillard's (Ed.) Brain and Space. Oxford University Press () 1991, Oxford, pp. 163182.

Poulton, E. C., (1974). Tracking Skill and Manual Control. Academic Press, New York, O 1974.

Regan, D (1997) Chapter 7: "Perceptual Motor Skills and Human Motion Analysis", in Salvendy (Ed.) Handbook of Human Factors, $2^{\text {nd }}$ Edition. John Wiley \& Sons, Inc. 0 1997, New York.

Roll, J.P., Roll, R., and Velay, J (1991). Chapter 7: "Proprioception as a link between body space and extra-personal space" in J. Paillard's (Ed.) Brain and Space. Oxford University Press () 1991, Oxford., pp. 112-132.

Roscoe, S.N., (1997). "Horizon Control Reversals and the Graveyard Spiral - Did a Human Control Reversal Cause the 1994 USAir Crash Near Pittsburgh?" CSERIAC GATEWAY, Vol. VI: No. 3. Crew System Ergonomics Information Analysis Center.

Roscoe, S.N. (1968). Airborne displays for flight and navigation. Human Factors, 10, 321-332.

Roscoe, S.N., and Williges, R.C. (1975). "Motion relationships in aircraft attitude and guidance displays: A flight experiment." Human Factors, 17, pp. 374-387.

Roscoe, S.N., Corl, L., and Jensen, R.S. (1981). "Flight display dynamics revisited." Human Factors, 23, 341-353. 
Rossano, M.J., and Warren, D.H. (1989). "Misaligned maps lead to predictable errors." Perception, 18, 215-229.

Senders, J.W. and Cruzen, M (1952). "Tracking performance on combined compensatory and pursuit tasks (WADC Tech. Rep. 52-39). Wright-Patterson Air Force Base, Ohio: Wright Air Development Center, February 1952.

Shepard, R. N., and Cooper, L.A. (1982). Mental images and their transformations. Cambridge, Mass.: M.I.T. Press Subject Bradford Books.

Shepard, R. N., and Metzler, J. (1971). "Mental rotation of three-dimensional objects." Science, 171, 701-703.

Salvendy, Gavriel (Ed.), (1987). Handbook of Human Factors. John Wiley \& Sons, Inc. O 1987, New York.

Salvendy, Gavriel (Ed.), (1997). Handbook of Human Factors, $2^{\text {nd }}$ Edition. John Wiley \& Sons, Inc. 0 1997, New York.

Shepard, R., and Hurwitz, S., (1984). "Upward direction, mental rotation and discrimination of left and right turn in maps". Chapter 4 in S. Pinker (Ed), Visual Cognition, Elsevier Science Publishers B.V., Amsterdam, The Netherlands, O 1984.

Sheridan, T. B., (1992). "Human Factors Considerations for Remote Manipulation, Advanced Guidance and Control Aspects in Robotics", AGARD Lecture Series 193, Ontario, Canada.

Stokes, A., Wickens, C., and Kite, K., (1990). Display Technology - Human Factors Concepts. 1990 Society of Automotive Engineers, Inc.

Tani, K, and Sheridan, T.B., (1983) "Compensation for Object Movement In Remote Manipulation". $19^{\text {th }}$ Annual Conference on Manual Control.

Vicente, K.J., \& Williges, R.C. (1998). "Accommodating individual differences in searching a hierarchical file system". International Journal of Man-Machine Studies, $29,647-668$.

Wettergreen, D., Bapna, D., Maimone, M., and Thomas, G. (1999). "Developing Nomad for robotic exploration of the Atacama Desert," Robotics and Autonomous Systems, 26: $127-148$.

Wickens, C.D. (1986). Chapter 39 "The effects of control dynamics on performance", in Boff, Kaufman \& Thomas (Ed.'s) Handbook of Perception and Human Performance. Wiley, 1986, 39-1-59.

Wickens, C.D. (1992). Engineering Psychology and Human Performance, Second Edition. HarperCollins Publishers 0 1992, New York. 
Wickens, C.D. (1995), "Integration of Navigational Information for Flight". Final Technical Report, October 1995. Aviation Research Laboratory, Institute of Aviation. University of Illinois at Urbana-Champaign.

Wickens, C.D., (1998) "Frame of Reference for Navigation". in D. Gopher \& A. Koriat (Eds.). Attention and performance. Vol. 16. Orlando, FL: Academic Press.

Wickens, C.D., and Carswell, C.M., (1997). Chapter 4: "Information Processing", in Salvendy (Ed.) Handbook of Human Factors, $2^{\text {nd }}$ Edition. John Wiley \& Sons, Inc. 0 1997, New York.

Wickens, C.D., Liang, C., Prevett, T., and Olmos, O., (1994). "Egocentric and Exocentric Displays for Terminal Area Navigation". Technical Report, January 1994. Aviation Research Laboratory Institute of Aviation, University of Illinois at UrbanaChampaign.

Yuille, J.C., and Steiger, J.H., (1982). "Nonholistic processing in mental rotation: Some suggestive evidence". Perception and Psychophysics. 31(3), 201-209.

Zhai, S. (1995). Human Performace In Six Degree of Freedom Input Control, PhD. Thesis. University of Toronto.

Zhai, S, and Milgram, P. (1994). Asymmetrical spatial accuracy in 3D tracking. In Proceedings of the Human Factors and Ergonomics Society $38^{\text {ih }}$ Annual Meeting (pp. 245-249). Santa Monica, CA: Human Factors and Ergonomics Society. 